\title{
AFRL-RQ-WP-TR-2016-0115
}

\section{MULTISCALE MODELING OF MULTIPHASE FLUID FLOW}

Soumya S. Patnaik

Flight Systems Integration Branch (AFRL/RQQI)

Power and Control Division

Eugeniya Iskrenova-Ekiert and Hui Wan

UES, Inc.

AUGUST 2016

Final Report

DISTRIBUTION STATEMENT A: Approved for public release.

Distribution is unlimited.

See additional restrictions described on inside pages

AIR FORCE RESEARCH LABORATORY

AEROSPACE SYSTEMS DIRECTORATE

WRIGHT-PATTERSON AIR FORCE BASE, OH 45433-7541

AIR FORCE MATERIEL COMMAND

UNITED STATES AIR FORCE 


\section{NOTICE AND SIGNATURE PAGE}

Using Government drawings, specifications, or other data included in this document for any purpose other than Government procurement does not in any way obligate the U.S. Government. The fact that the Government formulated or supplied the drawings, specifications, or other data does not license the holder or any other person or corporation; or convey any rights or permission to manufacture, use, or sell any patented invention that may relate to them.

This report was cleared for public release by the USAF 88th Air Base Wing (88 ABW) Public Affairs Office (PAO) and is available to the general public, including foreign nationals.

Copies may be obtained from the Defense Technical Information Center (DTIC) (http://www.dtic.mil).

AFRL-RQ-WP-TR-2016-0115 HAS BEEN REVIEWED AND IS APPROVED FOR PUBLICATION IN ACCORDANCE WITH ASSIGNED DISTRIBUTION STATEMENT.

*//Signature//

SOUMYA S. PATNAIK

Program Manager

Flight Systems Integration Branch

Power and Control Division
//Signature//

JACK U. VONDRELL, Chief

Flight Systems Integration Branch

Power and Control Division

Aerospace Systems Directorate

//Signature//

BRYAN J. CANNON, Principal Scientist

Power and Control Division

Aerospace Systems Directorate

This report is published in the interest of scientific and technical information exchange and its publication does not constitute the Government's approval or disapproval of its ideas or findings.

*Disseminated copies will show “//Signature//" stamped or typed above the signature blocks. 


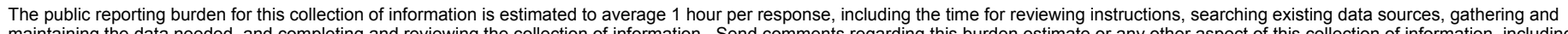

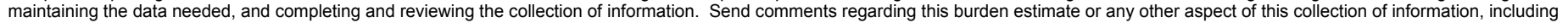

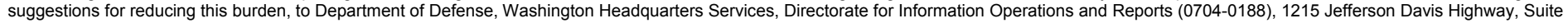

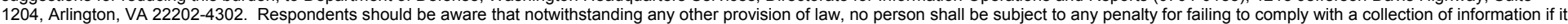
does not display a currently valid OMB control number. PLEASE DO NOT RETURN YOUR FORM TO THE ABOVE ADDRESS.
1. REPORT DATE (DD-MM-YY)
2. REPORT TYPE
August 2016
Final
3. DATES COVERED (From - To)
01 October 2014 - 30 January 2016

\section{TITLE AND SUBTITLE}

MULTISCALE MODELING OF MULTIPHASE FLUID FLOW

5a. CONTRACT NUMBER

In-house

5b. GRANT NUMBER

5c. PROGRAM ELEMENT NUMBER $61102 \mathrm{~F}$

\section{AUTHOR(S)}

Soumya S. Patnaik (AFRL/RQQI)

Eugeniya Iskrenova-Ekiert and Hui Wan (UES. Inc.)

5d. PROJECT NUMBER 3002

5e. TASK NUMBER

5f. WORK UNIT NUMBER

Q135

\section{PERFORMING ORGANIZATION NAME(S) AND ADDRESS(ES)}

Flight Systems Integration Branch (AFRL/RQQI) Power and Control Division

Air Force Research Laboratory, Aerospace Systems Directorate Wright-Patterson Air Force Base, OH 45433-7541

Air Force Materiel Command, United States Air Force
UES. Inc. 4401 Dayton-Xenia Rd. Dayton, $\mathrm{OH} 45432$
8. PERFORMING ORGANIZATION REPORT NUMBER

AFRL-RQ-WP-TR-2016-0115

9. SPONSORING/MONITORING AGENCY NAME(S) AND ADDRESS(ES)

Air Force Research Laboratory

Aerospace Systems Directorate

Wright-Patterson Air Force Base, OH 45433-7541

Air Force Materiel Command

United States Air Force

10. SPONSORING/MONITORING AGENCY ACRONYM(S)

AFRL/RQQI

11. SPONSORING/MONITORING AGENCY REPORT NUMBER(S)

AFRL-RQ-WP-TR-2016-0115

12. DISTRIBUTION/AVAILABILITY STATEMENT

DISTRIBUTION STATEMENT A: Approved for public release. Distribution is unlimited.

13. SUPPLEMENTARY NOTES

PA Case Number: 88ABW-2016-4374; Clearance Date: 07 September 2016.

The U.S. Government is joint author of this work and has the right to use, modify, reproduce, release, perform, display, or disclose the work.

14. ABSTRACT

A multiscale modeling approach including molecular dynamics and computational fluid dynamics was carried out address the disparate time and length scales involved in modeling fluid flow and heat transfer. Molecular dynamics simulations were carried out to provide a molecular level understanding of the transport properties of surfactant aqueous solutions; the solvent effects on the solubility of the thermal decomposition reaction products of ammonium carbamate; and the temperature dependence of the accommodation coefficient of liquid octane. Computational fluid dynamics methods were used to investigate the heat transfer process in open-cell micro-foam with phase change material; enhancement of natural convection for fluid media in an enclosure; and thermal effects on vortex-induced vibrations.

\section{SUBJECT TERMS}

Accommodation coefficient, Computational fluid dynamics, Heat transfer, Phase change material in Micro-foam, Molecular Dynamics, Multiphase flow, Multiscale modeling, Natural convection, Surfactant, Vortex-induced vibrations

16. SECURITY CLASSIFICATION OF:

a. REPORT

Unclassified

\section{b. ABSTRACT}

Unclassified c. THIS PAGE

Unclassified

\begin{abstract}
17. LIMITATION OF ABSTRACT: SAR
\end{abstract}

18. NUMBER
OF PAGES
132

19a. NAME OF RESPONSIBLE PERSON (Monitor) Soumya S. Patnaik

19b. TELEPHONE NUMBER (Include Area Code) $\mathrm{N} / \mathrm{A}$ 


\section{TABLE OF CONTENTS}

Section Page

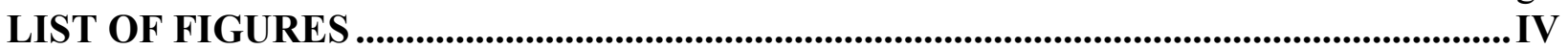

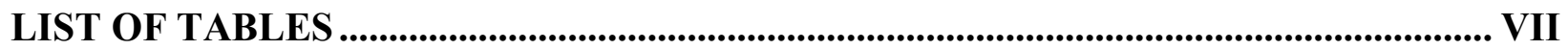

1 SUMMARY: MULTISCALE SIMULATIONS FOR POWER AND THERMAL

SYSTEMS MANAGEMENT ............................................................................................ 1

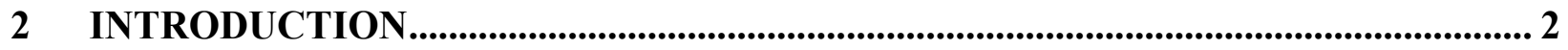

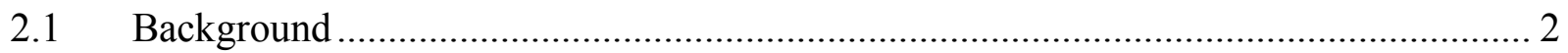

2.1.1 Heat Transfer Enhancement in Flows with Phase Change ............................................ 2

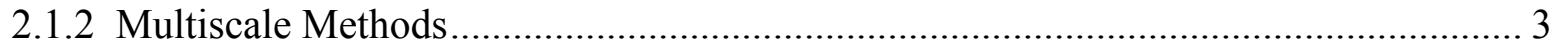

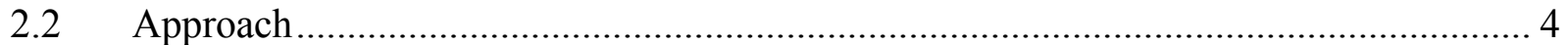

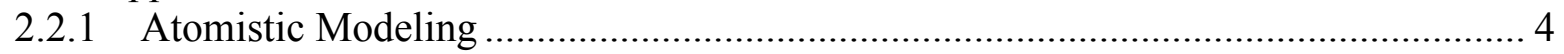

2.2.2 Continuum Approach...................................................................................... 4

3 TRANSPORT PROPERTIES OF WATER AND SODIUM DODECYL SULFATE

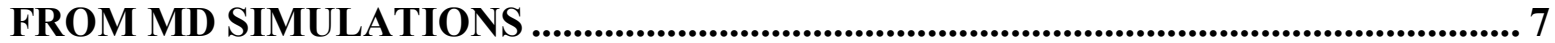

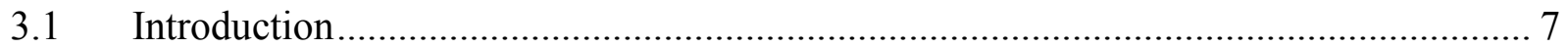

3.2 Transport Properties of Aqueous Surfactant Solutions at Room and Boiling

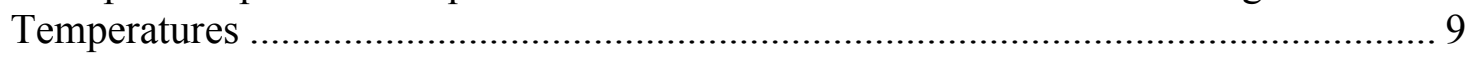

3.2.1 Surfactant Model and Simulation Details ............................................................. 9

3.2.2 Diffusion of SDS in Water: Results and Discussion .......................................... 10

3.2.3 Thermal Conductivity of Aqueous SDS Solutions: Results and Discussion............. 12

3.3 MP2f Water Model and Simulation Details......................................................... 13

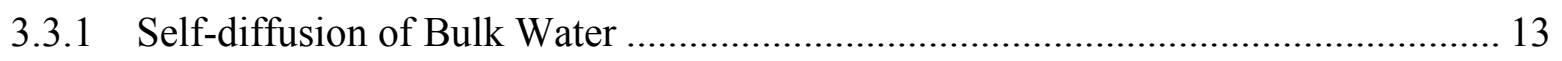

3.3.2 Self-diffusion and Thermal Conductivity of MP2f Water Model: Results and

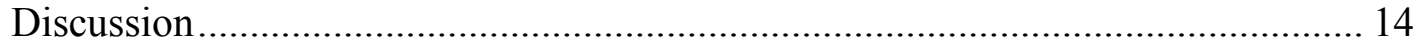

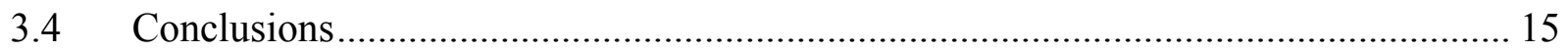

4 SOLVENT EFFECTS IN THE THERMAL DECOMPOSITION REACTION OF

AMMONIUM CARBAMATE......................................................................................... 17

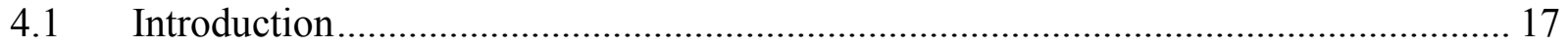

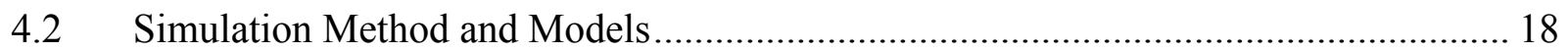

4.2.1 Solvents: Ethylene Glycol and Water ................................................................ 19

4.2.2 Decomposition Products: Carbon Dioxide and Ammonia....................................... 20

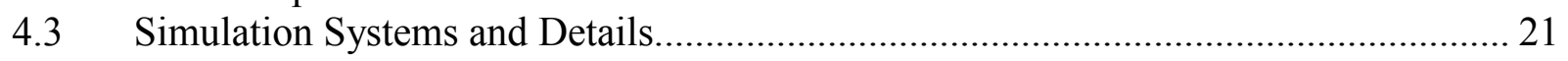

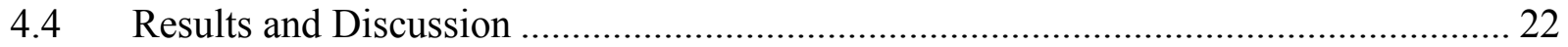

4.4.1 Equilibrium Volume and Equilibrium Volume Expansion .................................... 22

4.4.2 Temperature and Concentration Dependence of $\mathrm{CO}_{2}$ and $\mathrm{NH}_{3}$ Diffusivity in Water-

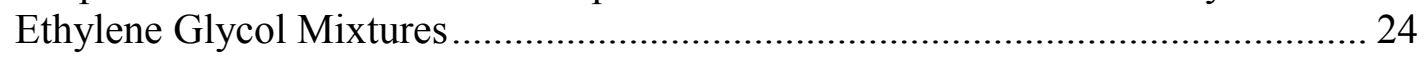

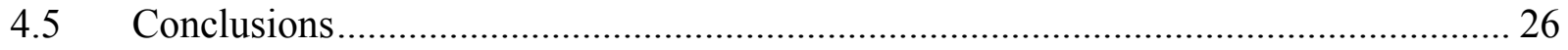

5 MOLECULAR DYNAMICS STUDY OF OCTANE CONDENSATION

COEFFICIENT AND EVAPORATIVE MASS FLUX AT LIQUID-VAPOR

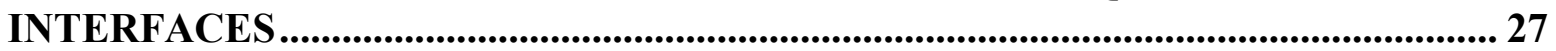

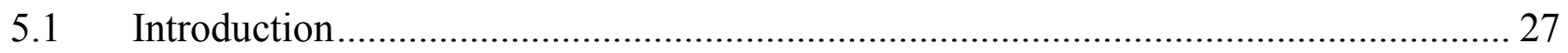

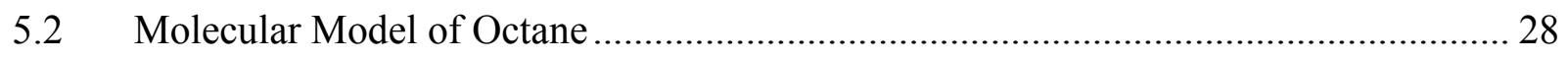

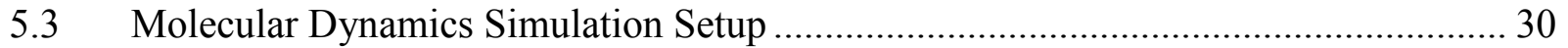

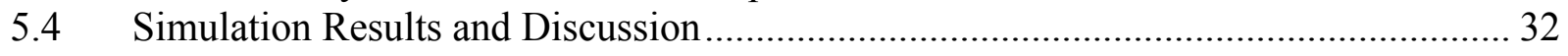

5.4.1 Structure of the Liquid-vapor Interfaces ................................................................. 32

i

DISTRIBUTION STATEMENT A: Approved for public release. Distribution is unlimited. 
5.4.2 Condensation Probabilities, Evaporation Rates, and Scattering Events .................. 34

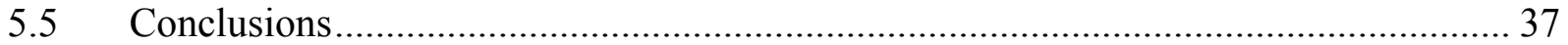

6 MODELING PHASE CHANGE MATERIAL IN MICRO-FOAM UNDER

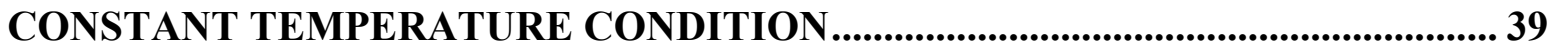

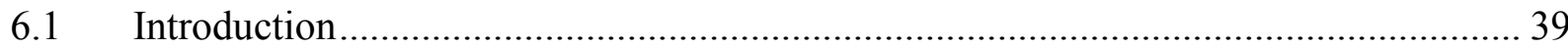

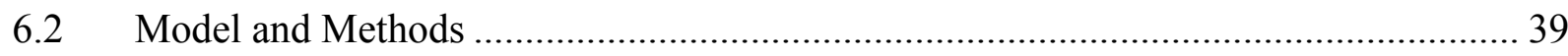

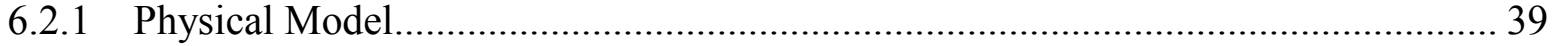

6.2.2 Direct Numerical Simulation of PCM in Foam .................................................. 40

6.2.3 One-temperature Simulation of Phase Change in Foam....................................... 41

6.2.4 Two-temperature Simulation of Phase Change in Foam ...................................... 41

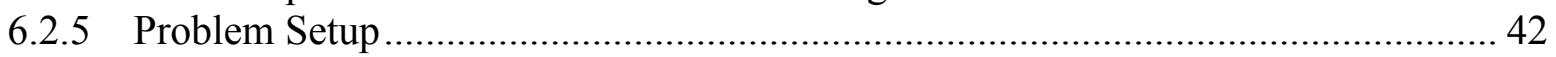

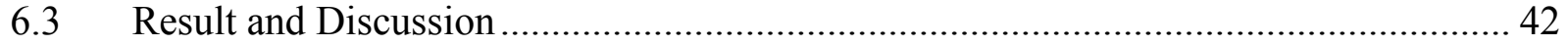

6.3.1 Volume Fraction of Melted PCM ................................................................... 42

6.3.2 Average Temperature at Different Y Cross Sections .......................................... 43

6.3.3 Temperature Difference between Micro-foam and PCM ...................................... 44

6.3.4 Heat Flux at Top Surface ........................................................................ 45

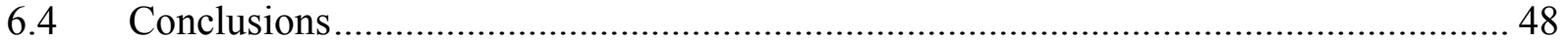

7 NUMERICAL MODELING OF HEAT TRANSFER IN OPEN-CELL MICRO-FAM

WITH PHASE CHANGE MATERIAL ......................................................................... 50

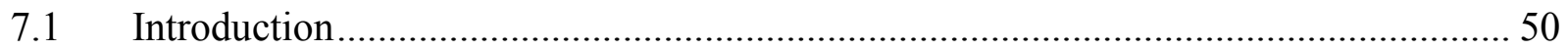

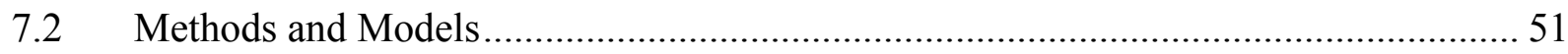

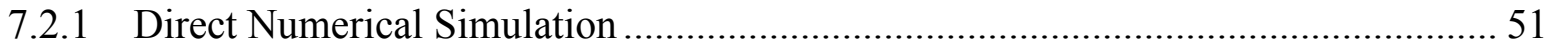

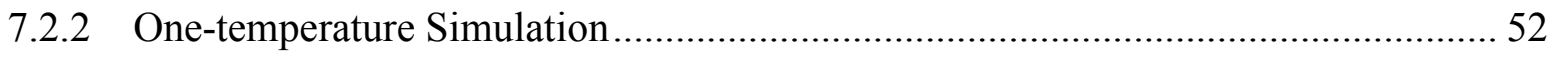

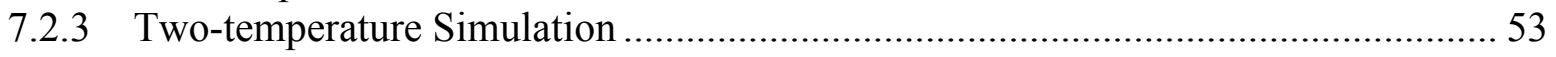

7.2.4 Effect of natural convection in low-porosity micro-foam .................................. 54

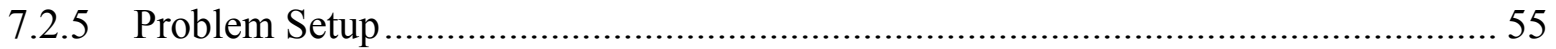

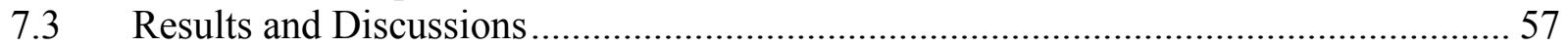

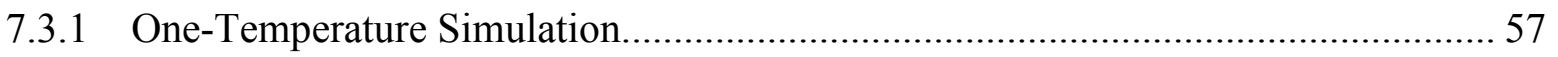

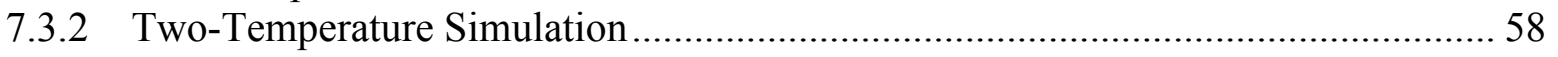

7.3.3 Direct Numerical Simulation ........................................................................ 59

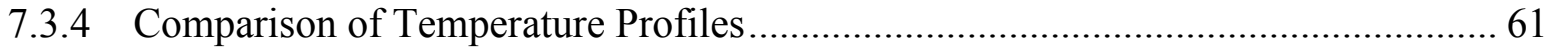

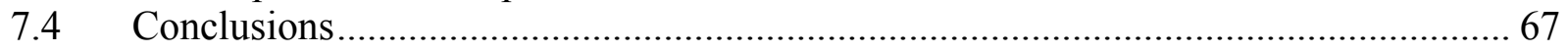

8 NATURAL CONVECTION IN A VERTICAL SQUARE ENCLOSURE WITH

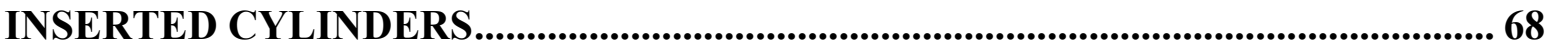

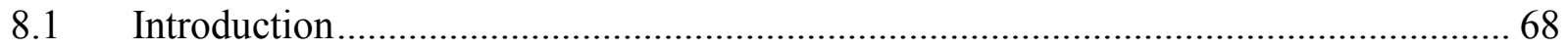

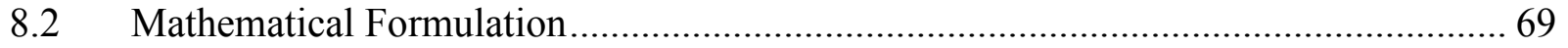

8.3 Numerical Method and Validation......................................................................... 70

8.3.1 Numerical Method ....................................................................................... 70

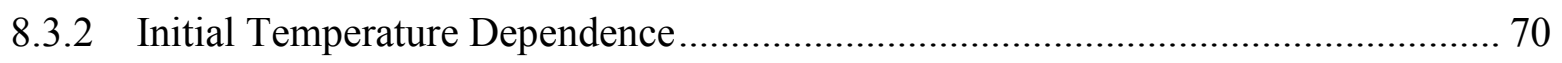

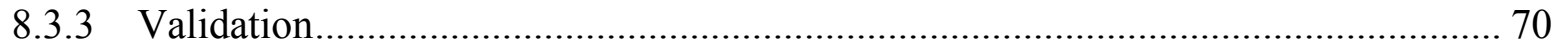

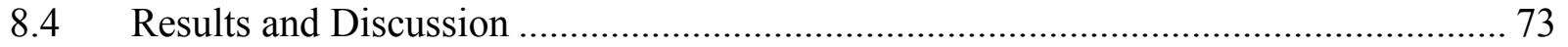

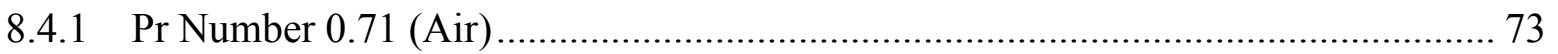

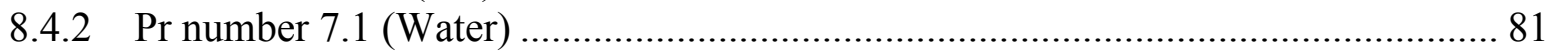

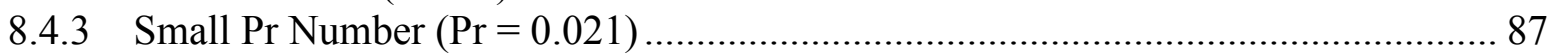

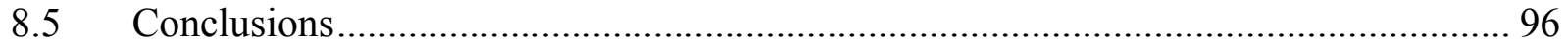

ii

DISTRIBUTION STATEMENT A: Approved for public release. Distribution is unlimited. 
9 THERMAL EFFECTS ON VORTEX-INDUCED VIBRATIONS ............................ 97

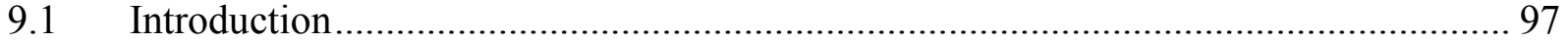

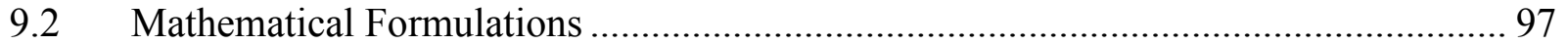

9.3 Numerical Method and Validation...................................................................... 98

9.3.1 Numerical Method ....................................................................................... 98

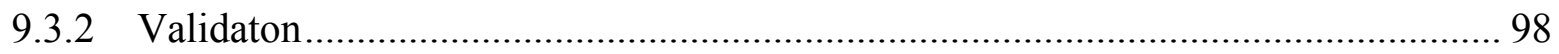

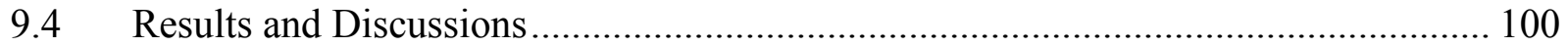

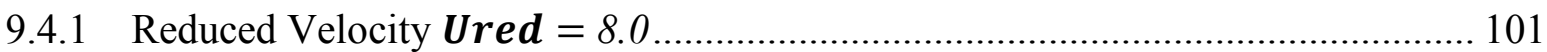

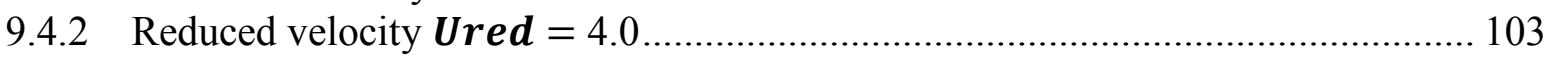

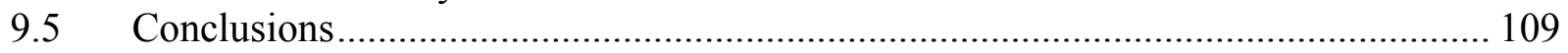

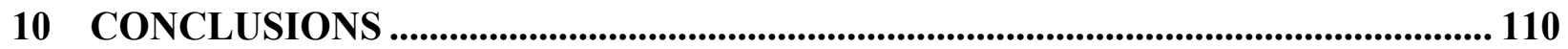

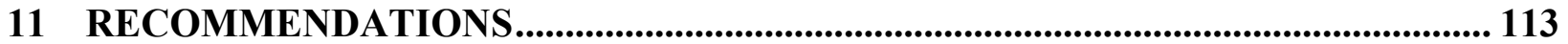

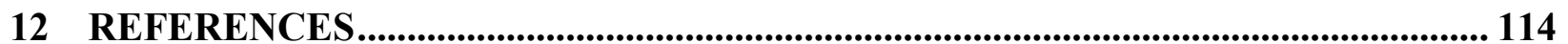




\section{LIST OF FIGURES}

Figure 2-1: Bubble Microlayer during Nucleate Boiling....................................................2

Figure 2-2: Multiscale Concept in Boiling ............................................................................. 3

Figure 2-3: Capabilities of the In-house High-fidelity CFD/CHT Solver ..................................6

Figure 3-1: Molecular Models of SDS...........................................................................

Figure 3-2: Diffusion Coefficient of SDS at Different Surfactant Concentrations at 373 K........11

Figure 3-3: Diffusion Coefficient as a Function of the Inverse Length of the Periodic

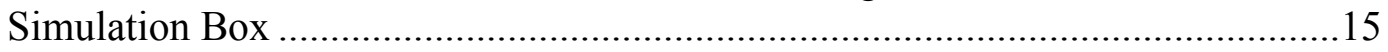

Figure 4-1. Atomic Structure of a Single Molecule of Ethylene Glycol ....................................19

Figure 4-2. Molecular Structure and Atom Labels of a Carbon Dioxide Molecule (left) and an Ammonia Molecule (right) .....................................................................21

Figure 4-3: $\mathrm{CO}_{2}$ and $\mathrm{NH}_{3}$ Equilibrium Molar Volume Expansion as a Function of the Gas Mole Fraction in Water (Circles) and Ethylene Glycol (Triangles) at 300 K. ......23

Figure 4-4: Concentration Dependence of the Equilibrium Molar Volume Expansion of

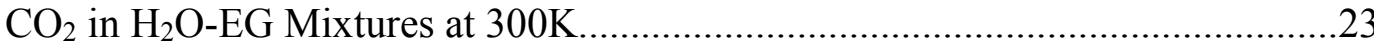

Figure 4-5: Equilibrium Molar Volume Expansion of $\mathrm{CO}_{2}$ and $\mathrm{NH}_{3}$ in $\mathrm{H}_{2} \mathrm{O}$ and $\mathrm{EG}$..............24

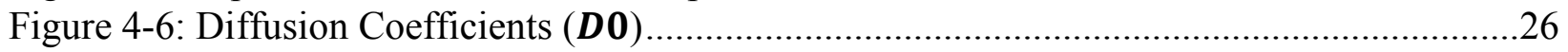

Figure 5-1: All-Atom and United-Atom Models of an Octane Molecule ..................................28

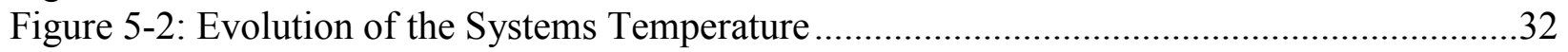

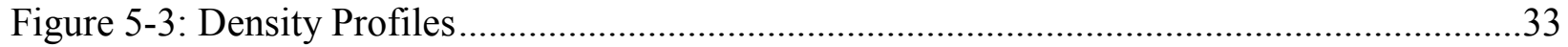

Figure 5-4: Time Evolution of the Z-coordinates of the Incident Molecules .............................34

Figure 5-5: Normalized Distributions of the Computed Accommodation Coefficient .................36

Figure 5-6: Macroscopic Accommodation Coefficient .........................................................37

Figure 6-1: Geometry and Microstructure of the Simulated Micro-Foam ................................40

Figure 6-2: Profiles of Melted PCM Volume Fraction (a) $\mathrm{T}_{\text {top }}=334 \mathrm{~K}$; (b) $\mathrm{T}_{\text {top }}=368 \mathrm{~K} \ldots \ldots \ldots . . .43$

Figure 6-3: Average Temperature Profiles .......................................................................4

Figure 6-4: Comparison of Temperature Difference between Micro-foam and PCM $\left(\mathrm{T}_{\text {top }}=\right.$

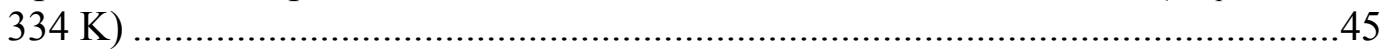

Figure 6-5: Comparison of Temperature Difference between Micro-foam and PCM $\left(\mathrm{T}_{\text {top }}=\right.$

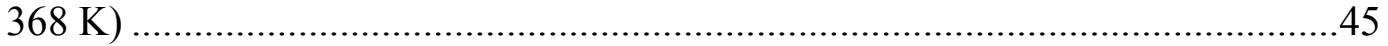

Figure 6-6: Heat Flux at Top Surface ..................................................................................47

Figure 6-7: Heat Flux at Top Surface (a) Overall (b) Individual Component $($ Ttop $=368$

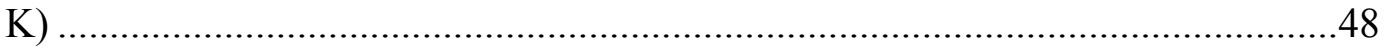

Figure 7-1: Effective Thermal Conductivity as a Function of Porosity ...................................52

Figure 7-2: Relationship between the Local Nusselt and Peclet Numbers .................................54

Figure 7-3: Pressure-Drop Rises with Increased Inlet Velocity ..............................................54

Figure 7-4: Physical Model Used for (a) DNS and (b) Volume-averaged Simulations ................56

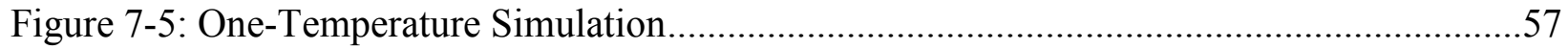

Figure 7-6: Difference in Average Temperatures of Micro-foam and PCM .............................58

Figure 7-7: Evolution of a 3-D Melting Front and Temperature Distribution..............................59

Figure 7-8: Temperature Distribution in the PCM/Foam Structure........................................60

Figure 7-9: Difference in Average Temperature of Micro-Foam and PCM..............................60

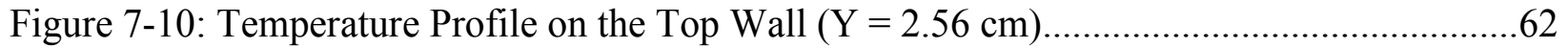

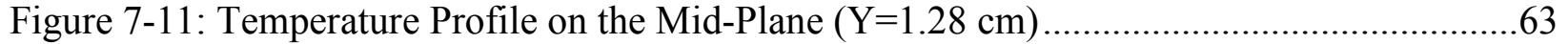

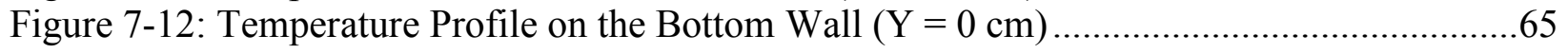

iv

DISTRIBUTION STATEMENT A: Approved for public release. Distribution is unlimited. 
Figure 7-13: Liquid PCM Volume Fraction Profile ………………………………....................66

Figure 8-1: Temperature Field Dependence on Initial Conditions ...............................................70

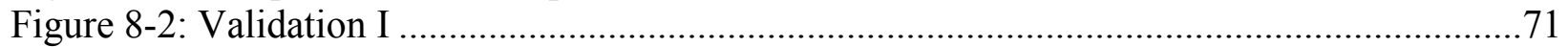

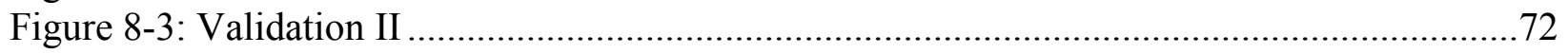

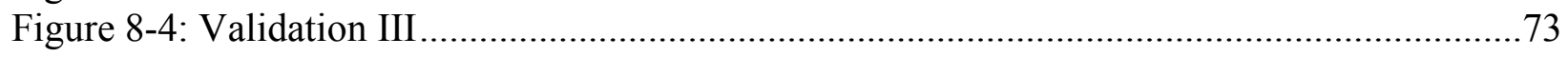

Figure 8-5: Surface-averaged Nusselt Number $\boldsymbol{N u} \boldsymbol{A}$ and Isotherms for $P r=0.71$ and $R a=$

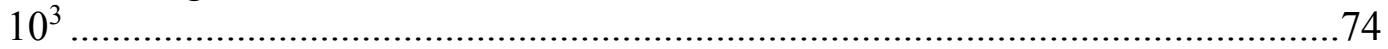

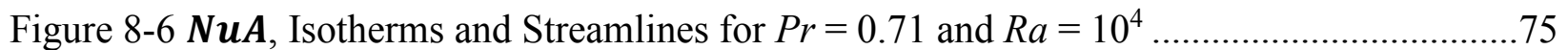

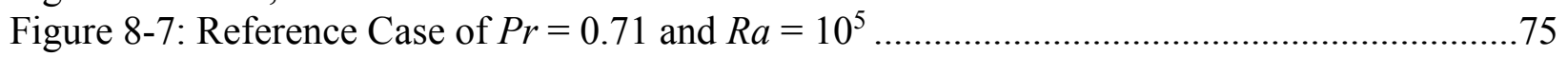

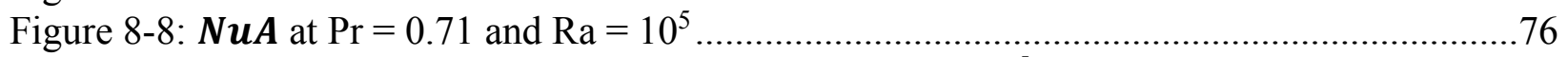

Figure 8-9: Isotherms and Streamlines at $\operatorname{Pr}=0.71$ and $\mathrm{Ra}=10^{5}$, with One Inserted

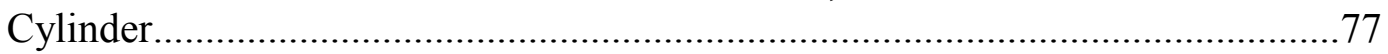

Figure 8-10: $\boldsymbol{N u} \boldsymbol{A}$ and Isotherms at $\operatorname{Pr}=0.71$ and $\mathrm{Ra}=10^{5}$, with Two Inserted Cylinders .........77

Figure 8-11: $\mathbf{N u A}$ at $\operatorname{Pr}=0.71$ and $\mathrm{Ra}=10^{5}$, with Three Inserted Cylinders ............................78

Figure 8-12: Isotherms and Streamlines at $\operatorname{Pr}=0.71$ and $\mathrm{Ra}=10^{5}$, with Three Inserted

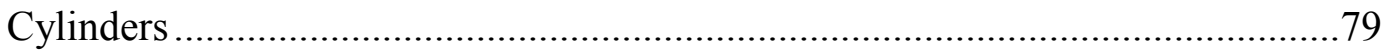

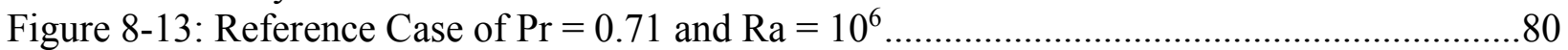

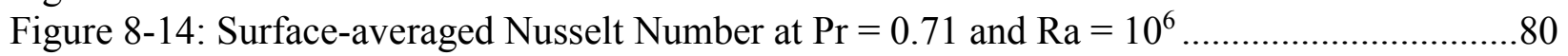

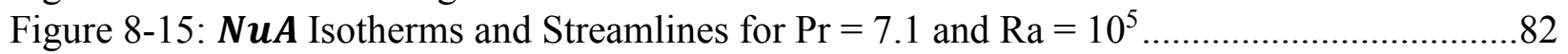

Figure 8-16: Nusselt Number Distribution at the Bottom (hot) and Top (cold) Surfaces ..............83

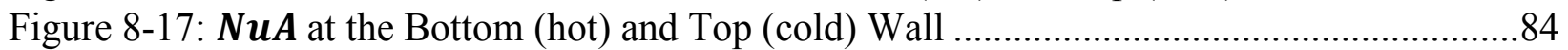

Figure 8-18: Isotherms and Streamlines for the Reference Case of $\operatorname{Pr}=7.1, \mathrm{Ra}=10^{6} \ldots \ldots \ldots \ldots \ldots . . .84$

Figure 8-19: Nusselt Number at the Bottom (hot) and Top (cold) Wall, One Cylinder in a

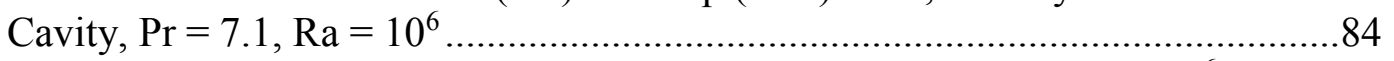

Figure 8-20: Isotherms and Streamlines in a One-cylinder Enclosure, $\operatorname{Pr}=7.1, \mathrm{Ra}=10^{6} \ldots \ldots \ldots . .85$

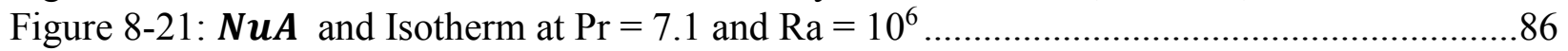

Figure 8-22: Surface-averaged Nusselt Number, Isotherms, and Streamlines at $\operatorname{Pr}=0.021$,

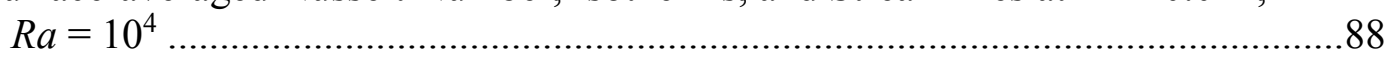

Figure 8-23: $\boldsymbol{N u A}$ at the Bottom Surface for the Reference Case at $P r=0.021$ and $R a=$

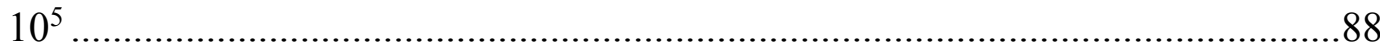

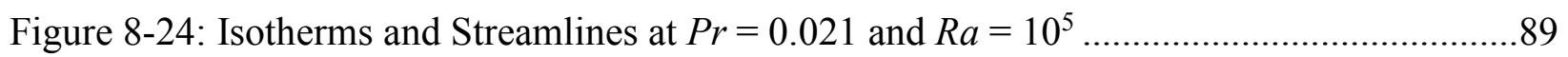

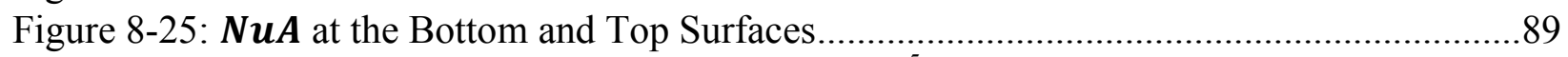

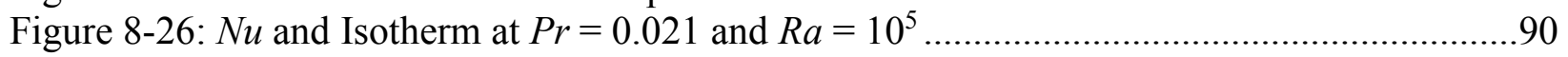

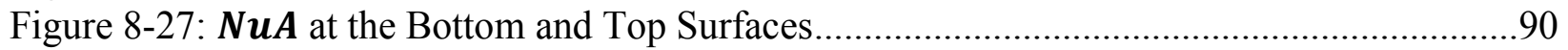

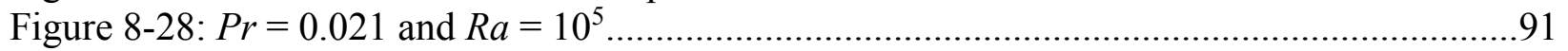

Figure 8-29: Isotherms and Flow Structure at Time Instants Corresponding to Figure 8-28,

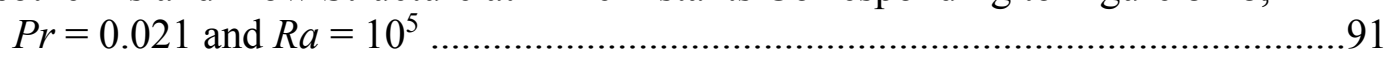

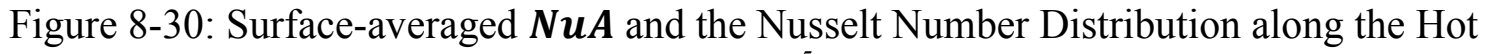

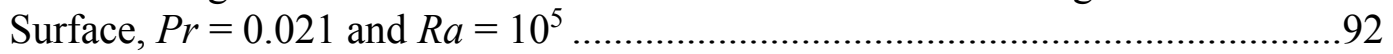

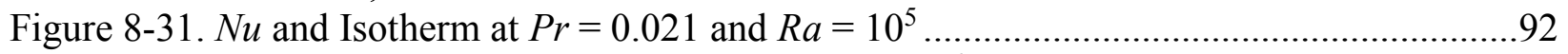

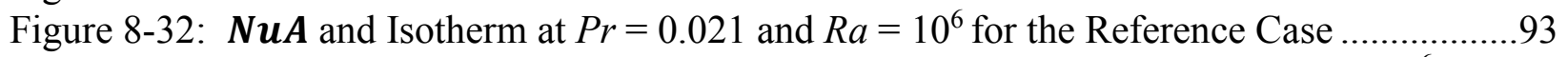

Figure 8-33. Isotherms and Streamlines of the Reference Case at $P r=0.021$ and $R a=10^{6} \ldots \ldots . .93$

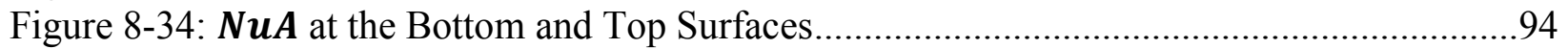

Figure 8-35: Surface-Averaged Nusselt Number Showing Periodic Oscillation ............................95

Figure 8-36: Time-averaged Isotherms and Streamlines ...........................................................95

Figure 9-1: Phase Changes between Lift Coefficient and Vibration Amplitude, Mred = 2 .......99 
Figure 9-2: Vortex Structure at Extreme Positions (2S mode), $R e=150$, Mred $=2.0$,

$$
\text { Ured }=8.0
$$

Figure 9-3: Vortex Structure at Extreme Positions, $R e=150$, Mred $=2.0$, Ured $=4.0 \ldots \ldots \ldots . .100$

Figure 9-4: Time History of the Displacement of Cross-stream Vibration, $R i=0$.....................101

Figure 9-5: Vortex Development at Certain Time Instants, Ured $=8.0, R i=0 \ldots \ldots \ldots \ldots \ldots \ldots \ldots \ldots . . .101$

Figure 9-6: Vortex Structure at Extreme Position, for Various Ri numbers, Ured = 8.0 ….....102

Figure 9-7: Time History of the Displacement ....................................................................... 102

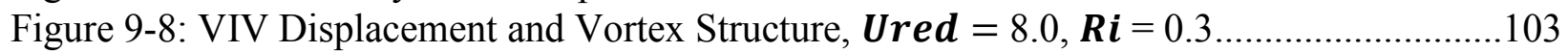

Figure 9-9: Time History of the Displacement of Cross-stream Vibration, Ured $=\mathbf{4 . 0}$, $\mathbf{R i}=\mathbf{0}$

Figure 9-10: Vortex Structure after a VIV Cylinder at Various Locations, Ured $=\mathbf{4 . ~} 0$, $\mathrm{Ri}=\mathbf{0}$ 104

Figure 9-11: Time History of Displacement of Cross-stream Vibration, Ured $=\mathbf{4 . 0}, R i=$ 0.3 .

Figure 9-12: Vortex Structure at Various Locations, Ured $=$ 4. 0, $R i=0.3$ 105

Figure 9-13: Time History of Displacement of Cross-stream Vibration, Ured $=\mathbf{4 . ~ 0 , R i}=$ $0.5,0.7$ and 0.8

Figure 9-14: Development of Vortex Structure, Ured $=4.0, R i=0.7$

Figure 9-15: Development of Temperature Contour at Ured $=4.0, R i=0.7$

Figure 9-16: Flow Past a VIV Cylinder, Ured $=4.0, R i=0.8, t=500$ 


\section{LIST OF TABLES}

Table 3-1: Diffusion Coefficient $\mathrm{D}_{\mathrm{s}}$ of Aqueous SDS Solutions at $298.15 \mathrm{~K}$

Table 3-2: Thermal Conductivity $\boldsymbol{\lambda}[\boldsymbol{W m} \cdot \boldsymbol{K}]$ of Aqueous SDS Solutions at $298.15 \mathrm{~K}$ and $373 \mathrm{~K}$

Table 3-3: Size-independent Diffusion Coefficient $\left(\mathrm{D}_{0}\right)$ and Viscosity $(\boldsymbol{\eta})$ of MP2f Water Model at $298.15 \mathrm{~K}$ and $373 \mathrm{~K}$

Table 3-4: Computed Thermal Conductivity $\lambda$ in $\mathrm{W} /(\mathrm{m} \cdot \mathrm{K})$ of Neat Water at Room and

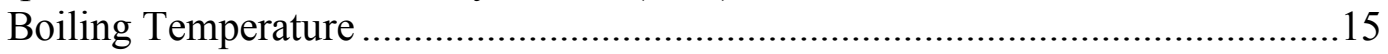

Table 4-1. Values of the Force Field Parameters for Ethylene Glycol ......................................20

Table 4-2. Non-bonded Parameters for Carbon Dioxide ...........................................................21

Table 4-3. Non-bonded Parameters for Ammonia..................................................................21

Table 4-4. Number of Gas Molecules $\left(\mathrm{CO}_{2}\right.$ or $\left.\mathrm{NH}_{3}\right)$ in the Simulation Systems........................22

Table 4-5: Values of the Computed Diffusion Coefficients of $\mathrm{CO}_{2}$ and $\mathrm{NH}_{3}$ in Water-EG

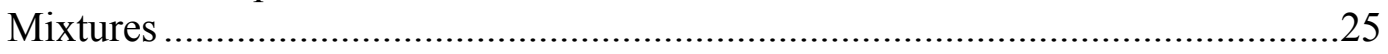

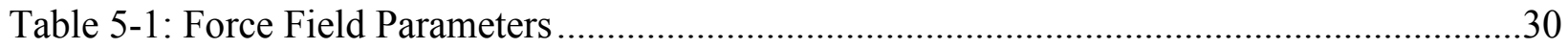

Table 5-2: Liquid Densities, in $\mathrm{g} / \mathrm{cm}^{3}$, Computed at the Five Simulation Temperatures ..............33

Table 5-3: Interfacial Widths, in $\AA$, Computed at the Five Simulation Temperatures..................33

Table 5-4: Summary of the Total Number of Observed Incident Molecules $\mathrm{N}_{\text {inc, }}$

Evaporation $\left(\mathrm{N}_{\text {evap }}\right)$ and Condensation $\left(\mathrm{N}_{\text {cond }}\right)$ Events, and Total Observation

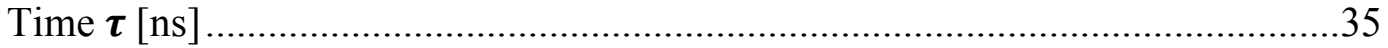

Table 5-5: Macroscopic Accommodation Coefficient ( $\boldsymbol{\alpha c})$ and Evaporative Mass Flux of

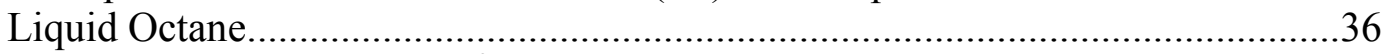

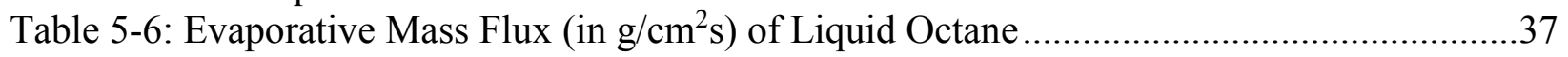

Table 6-1: Thermo-Physical Parameters for PCM and Micro-foam ..........................................40

Table 7-1: Permeability and Inertial Coefficient at Different Micro-Foam Porosities.................55

Table 7-2: Thermophysical Parameters for PCM and Micro-foam .........................................56

Table 8-1: Comparison on Nusselt Number for a Square Cavity with Lateral Temperature

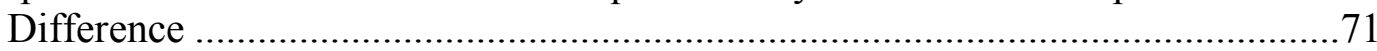

Table 8-2: $\boldsymbol{N u} \boldsymbol{A}$ at the Hot Surface for $\mathrm{Pr}=0.71$, at Various Rayleigh Numbers.......................81

Table 8-3: Nusselt Number at the Hot Surface for $\operatorname{Pr}=7.1$, at Various Ra, and Number of

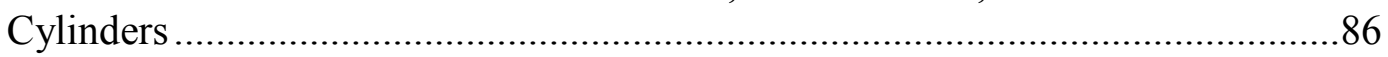

Table 8-4: Nusselt Number at $P r=0.021$, Various $R a$, and Number of Cylinders....................96

Table 9-1: Comparison on Nusselt Number for a Square Cavity with Lateral Temperature

Difference 


\section{SUMMARY: MULTISCALE SIMULATIONS FOR POWER AND THERMAL SYSTEMS MANAGEMENT}

Thermal management challenges on emerging USAF aircraft have been recognized to be a limiting factor in providing future capabilities, therefore requiring consolidated attention from the DoD Scientific Community. With the arrival of high-power electronics, high-efficiency engines, and greater capability demands from the Warfighter, current cooling solutions are no longer capable of addressing the unprecedented thermal demands and development of novel high efficiency cooling solutions is critical.

Multiphase fluid flow with its high heat transfer is, therefore, of great interest in the thermal management of military aircraft with stringent cooling requirement of very high heat loads, fast cooling needs, and limited payloads. Apart from aircraft thermal management, multiphase convective boiling heat transfer and heat storage that can sustain high heat fluxes for efficient heat removal have wider applications in thermal management of airborne systems and microelectronics. These thermal management systems are based on a variety of promising approaches such as latent heat-based thermal energy storage, two-phase vapor cycle systems, boiling in microchannels, jet impingement, and spray-cooling that use the latent heat of phase change for evaporation (and condensation) or fusion providing high efficiencies. The heat transfer provided by these approaches can be further augmented by nano-modification, such as inclusion of micro and nano channels to increase the contact surface, improvement in nucleation by introducing surface roughness and variable wettability, tailoring the solid/liquid interface by hydrophilic/hydrophobic surface treatment, nano-structured coatings, introduction of surfactants to control the surface tension, use of microfoam with phase change material for increased effective conductivity, and many more.

The combination of multiphase heat transfer and nano-technology, thus, opens up a wide range of possibilities for cooling technologies that is yet to be explored. To better exploit the added benefits provided by combining these two very intensive but diverse technology areas, the underlying physics of heat transfer and interfacial effects that spans across all these technologies has to be well understood and needs long term scientific investments including research in modeling approaches. Due to the complex multi-physics mechanisms involved and the need for complete understanding of flow boiling and the effect of interfacial properties on heat transfer, analytical models for multiphase heat transfer are limited.

Our research effort has been aiming at addressing some of these limitations by building expertise and developing numerical simulation approaches handling the disparate time and length scales involved in modeling fluid flow and heat transfer. In this report, we summarize our efforts in molecular dynamics (MD) and computational fluid dynamics (CFD) simulations of fluid flow. Within the MD effort, our recent focus has been on developing approaches to incorporate molecular properties calculated from MD simulations of fluids and liquid-vapor interfaces into continuum models by refining the macroscopic parameters used in the continuum description of multi-phase fluid flow. In particular, we have investigated the transport properties of surfactant (sodium dodecylsulfate) aqueous solutions, the solvent effects on the solubility of the products of the thermal decomposition reaction of ammonium carbamate used as a novel heat sink in a heat exchanger, and the temperature dependence of the accommodation coefficient of liquid octane for the purposes on modeling cooling by thin film evaporation. Within the CFD effort, we have numerically studied heat transfer process in open-cell micro-foam with phase change material. We also studied enhancement of natural convection for various fluid media in an enclosure, and thermal effects on vortex-induced vibrations, which is a thermal-fluid-structure coupled problem.

DISTRIBUTION STATEMENT A: Approved for public release. Distribution is unlimited. 


\section{INTRODUCTION}

\subsection{Background}

\subsubsection{Heat Transfer Enhancement in Flows with Phase Change}

The current understanding of fundamental flow boiling is still very limited. Boiling and condensation which are the foundation of most phase change based cooling technologies include complex mechanisms such as nucleation, merger and break up of vapor-liquid interfaces, contact line behavior, and bubble growth (Figure 2-1).

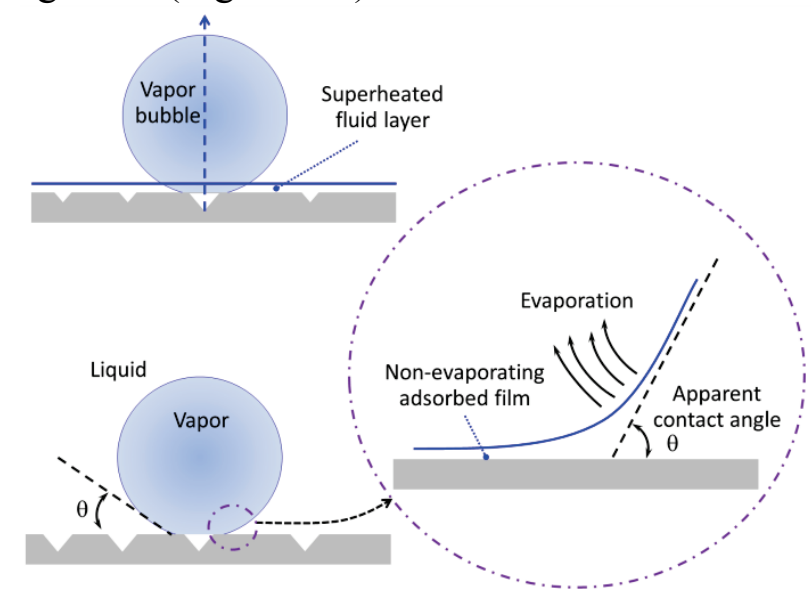

Figure 2-1: Bubble Microlayer during Nucleate Boiling

In nucleate boiling, the formation of bubbles and their departure lead to heat transfer that is directly controlled by the evaporation at the microlayer underneath the bubble and around the bubble and the convection created from buoyancy. The net heat transfer, therefore, depends on the number density, bubble size at departure, and bubble merger and break up processes. The nucleation site density also plays an important role on the rate of heat transfer from the heated surface and the structures of the phases near the heated wall.

Although it is accepted that the defects or cavities that trap gas are potential nucleation sites, the surface tension, contact angle and shape of the cavity, all influence the distribution of these sites. The behavior of the contact line is yet another determining factor for heat transfer. As a vapor bubble grows on a nucleation site, a very thin microlayer forms underneath it which is controlled by the molecular nature of the surface. All these clearly point to the significance of the molecular origin of the phase change process and also the associated mechanisms. To summarize, flow and heat transfer during boiling are governed by interfacial phenomena at the liquid vapor, liquid-solid and solid-vapor interfaces. These manifest via interfacial tension, surface energy, surface wettability, surface roughness, advancing/receding contact angle, interfacial forces, and phase change. The processes that govern these interfacial phenomena occur at molecular scale. However, the bubble nucleation $\rightarrow$ growth $\rightarrow$ departure takes place at millimeter scale which then governs the flow hydrodynamics and heat transfer at macro-scale (Figure 2-2). Although boiling and condensation have been investigated extensively leading to this increased understanding of the processes, a lot of the research has focused on development of correlations and semi-theoretical models for intended applications. These correlations have mostly relied on empirical data and models that are loosely tied to the underlying physics and therefore have limited transferability.

DISTRIBUTION STATEMENT A: Approved for public release. Distribution is unlimited. 

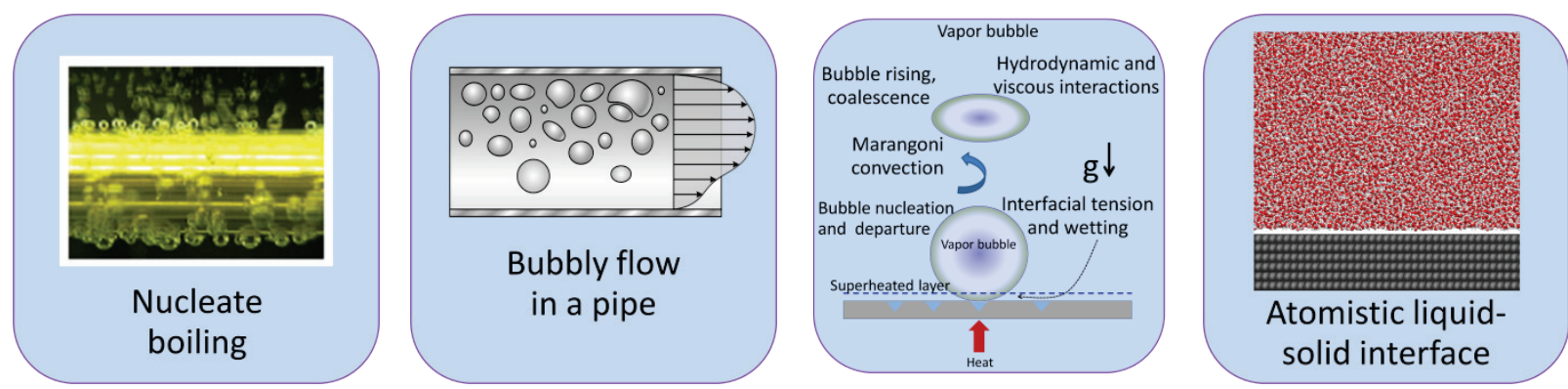

Figure 2-2: Multiscale Concept in Boiling

(From left to right: Experimental observation of nucleating bubbles on a hot surface in liquid [1]; schematic of mesoscopic bubbly flow in a pipe; bubble growth on heated solid surface; atomistic representation of a solid-liquid interface.)

\subsubsection{Multiscale Methods}

Although clearly a multiphysics and multiscale problem, until recently, numerical modeling of the phase change problem in heat transfer has been mostly based on continuum approaches. Traditional CFD methods which solve the Navier-Stokes equations and start from discretizing the governing partial differential equations by finite volume, finite element or finite difference methods have been very successful in modeling a wide variety of flows; however, this macroscopic approach has limitations while addressing many of the complex mechanisms described earlier. The need for developing a multiscale approach that can address the interfacial effects is even greater when nano-modifications for heat transfer enhancement are being studied. The continuum-based methods have also not been found to be very accurate for more general problems involving flows with non-Newtonian fluids; complex boundaries; significant microscopic interactions or high Knudsen number including micro and nano-fluidics. In particular, nanodevices, microfluids and nanofluids demand better understanding and accounting of the atomistic details at the interfaces because of increased interfacial effects where assumptions of the continuum description of fluids are no longer valid [1] and the thermal fluctuations can no longer be neglected [2]. This effort aims at addressing some of these limitations of continuum based CFD methods by introducing a multiscale framework for a hybrid MD and CFD approach to modeling heat transfer in fluid flow. MD simulations have long shown great promise as tools in statistical mechanics and materials science [3-6] and are recently becoming more practical for heat transfer problems [7-10]. Although atomistic molecular dynamics can provide insight into the molecular nature of heat transfer, the spatial and timescales accessible by MD are of the order of tens of nanometers and hundreds of nanoseconds and therefore not practical neither sufficient to model macro-scale processes. However, a combined CFD and MD hybrid approach can address the two disparate time scales, and, in this effort we use a CFD formulation to heat transfer and augment it with MD. In particular, we have investigated the role of surface effects (surface energy, wettability and interfacial forces) and their modification via surface active reagents on boiling and two phase flow and the corresponding heat transfer behavior. Our recent focus [10-13] has been in supporting modeling approaches for multiscale processes which have unique challenges compared to multiscale systems. For multiscale heat transfer processes that have significant contribution from both conduction and convection, modeling approaches that are geared towards multiscale systems dominated by conduction are not very applicable and specific approaches have to be developed that capture both conduction and convection.

DISTRIBUTION STATEMENT A: Approved for public release. Distribution is unlimited. 


\subsection{Approach}

\subsubsection{Atomistic Modeling}

In classical MD simulations, Newton`s equations of motion are numerically solved for each atom in the potential field created by the rest of the atoms in the system [14]. The long-range intermolecular interactions in an atomistic system are the Lennard-Jones (repulsive short-range $\mathrm{R}^{-}$ 12 and attractive long-range $\mathrm{R}^{-6}$ interactions) and Coulomb interactions between any particles having charge. Each atom and its interactions with the rest of the atoms in the system are described by atom-type specific parameters, also referred to as the force field (FF) parameters of the simulation. Bulk properties are modeled by imposing periodic boundary conditions on the simulated system. The usual size of the atomistic systems accessible by MD is hundreds of nanometers and the usual timescale of the simulations is hundreds of nanoseconds to, in special coarse-grained cases, microseconds $[4,15]$.

Atomistic modeling approach and MD simulations were used in the studies described in Section 3, Section 4, and Section 5.

\subsubsection{Continuum Approach}

Advanced computation methodologies have been developed for moving boundary problems during the past decades. There are generally four ways to characterize inherent moving boundaries or interfaces in computational fluid dynamics. First method is the Front tracking or Immersed boundary method, in which moving boundaries $\Gamma(t)$ are discretized by Lagrangian marker/tracer points, whose position and velocity can be either prescribed [16] or obtained through fluid-body interactions [17]. When handling multiphase problems, the physical interface is a functional interface of zero thickness and physical quantities such as density and viscosity are discontinuous at the interface.

The second method is the volume-of-fluid (VOF) method [18], which represents the interface by the cell volume or surface fraction. VOF methods have the advantage of conserving mass by explicitly tracking volume fractions of each cell, but reconstruction of interfaces from volume fraction is difficult. Another disadvantage of VOF is that it is hard to compute accurate local curvatures from volume fractions, especially, in three-dimensional simulations. Standard VOF method computes the curvature by appropriately smoothing the volume fractions.

The third is the Level set method $[19,20]$, in which the interface is implicitly defined by a level function $\phi$ and its zero-level set $\phi=0$ corresponds to the evolving front $\Gamma(t)$. The interface evolution is described by: $\phi_{t}+\mathbf{u} \cdot \nabla \phi=0$. In the level set method, the function $\phi$ is initialized as the signed distance from the interface and solved with high-order numerical discretization schemes in time and space. To keep the values of $\phi$ close to the signed distance function, a reinitialization step is needed either in each time step or every several time steps by solving a transient partial differential equation to steady state [21]. The main drawback of the level set method is that it cannot satisfy the volume conservation, because of errors from advection calculation or reinitialization. The CLSVOF (coupled level set and volume-of-fluid) method [22], VOSET (volume-of-fluid and level set) method [23], and conservative level set method [24] have been proposed to resolve the problems arisen from the VOF or/and level set method. The implementation of these schemes is more complicated than the original VOF or level set methods. Even with a frequent re-initialization step the level set method tends to lead to mass loss in long time simulations [25].

The fourth method is the Phase field method [26], in which the thin boundary or interface is replaced by a transition region of small but fine width. The physical properties across the

DISTRIBUTION STATEMENT A: Approved for public release. Distribution is unlimited. 
interface vary steeply but continuously. The traditional phase field methods employs a phase-field variable $\phi$, to describe whether the material is liquid or solid. The behavior of this variable is governed by an equation that is coupled to equations for heat and solute transport. Interfaces between different phases are described by smooth but highly localized changes of this variable between fixed values that represent solid and liquid [26]. Phase-field method has the following several advantages [27]. First: modeling of fluid interfaces as having finite thickness greatly simplifies the handling of topological change of the interface, which can merge or break up while no extra coding is required. Second, the composition field has physical meaning not only on the interface but also in the bulk phase. Hence, this method can be applied to many physical states such as miscible, immiscible, and partially miscible phases. Third, the method is able to simulate contact line motion as the stress singularity in the immediate vicinity of the contact line is removed. Fourth, the explicit tracking of the interface is unnecessary and all governing equations can be solved over the entire computational domain without any a priori knowledge of the location of the interfaces.

Our current in-house high fidelity CFD and computational heat transfer (CHT) solver is based on immersed-boundary sharp interface method [16, 28], with the functionality to handle both prescribed and flow-induced inner boundary-moving problems. The dimensionless NavierStokes equations for flow and heat transfer can be written as:

$$
\begin{aligned}
& \nabla \cdot \mathbf{u}=-\frac{1}{\rho} \frac{D \rho}{D t} \\
& \frac{\partial \mathbf{u}}{\partial t}+\mathbf{u} \cdot \nabla \mathbf{u}=\frac{1}{\rho}\left(-\nabla p+\frac{1}{R e} \nabla \cdot(2 \mu \overline{\bar{D}})+\frac{1}{W e} F_{s}\right)+\frac{1}{F r^{2}} \boldsymbol{e}_{\boldsymbol{g}} \\
& \frac{\partial T}{\partial t}+\mathbf{u} \cdot \nabla T=\nabla^{2} T \\
& \mathbf{F}(t)=m \frac{d \mathbf{v}_{\mathbf{c}}}{d t}, \boldsymbol{\tau}_{C}(t)=\boldsymbol{I} \cdot \frac{d}{d t} \dot{\theta}+\dot{\theta} \times(\boldsymbol{I} \cdot \dot{\theta})
\end{aligned}
$$

in which $\mathbf{u}$ is the velocity, $p$ is the pressure, Tis the temperature, $\rho$ is the fluid density, and $v$ is the kinematic viscosity, respectively. Re is the Reynolds number, $W e$ is the Weber number, and $\mathrm{Fr}$ the Froude number. The mass and moment of inertia of the solid body are denoted by $m$ and $\boldsymbol{I}$, and $\mathbf{V}_{\mathbf{c}}$ and $\dot{\theta}$ stand for the linear velocity and angular velocity of the body. The instantaneous force $\mathbf{F}(t)$ and torque $\boldsymbol{\tau}_{C}(t)$ applied on the body are obtained from surface integration of pressure and shear stress calculated from the Navier-Stokes equations. The Navier-Stokes equations are discretized using a cell-centered, collocated (non-staggered) arrangement of the primitive variables $(\mathbf{u}, p)$ and integrated in time using fractional step method. In the first step, a modified momentum equation is solved and an intermediate velocity is obtained. A second-order Adams-Bashforth scheme is employed for the convective terms while the diffusion terms are discretized using an implicit Crank-Nicolson scheme which eliminates the viscous stability constraint. The second step is to solve the pressure Poisson equation based on multigrid method. The last step is the update of velocity based on calculated pressure gradient. More details on numerical algorithm and immersed boundary treatment, as well as validations on the DNS solver can be found in Mittal et al. [16]. The current immersed-boundary method based CFD and heat transfer solver can handle both the prescribed motion and fluid-body interaction problems [17, 29-32].

DISTRIBUTION STATEMENT A: Approved for public release. Distribution is unlimited. 


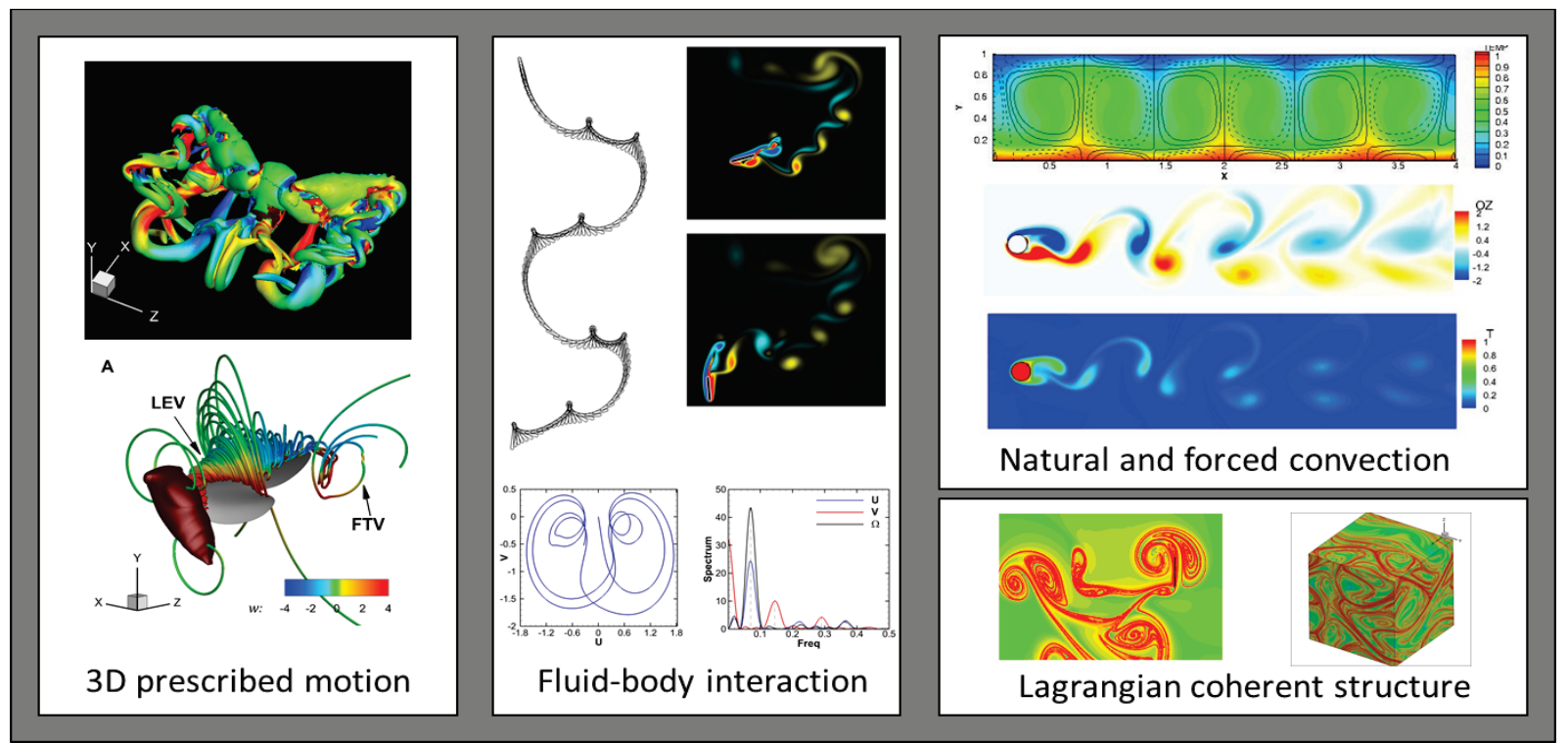

Figure 2-3: Capabilities of the In-house High-fidelity CFD/CHT Solver

(All pictures are generated using the in-house solver)

Figure 2-3 illustrates the capabilities of our in-house CFD solver. The continuous modeling approach and our in-house CFD/CHT solver were used in the studies described in Section 6, Section 7, Section 8, and Section 9. 


\section{TRANSPORT PROPERTIES OF WATER AND SODIUM DODECYL SULFATE FROM MD SIMULATIONS}

We performed an atomistic molecular dynamics study investigating the effect of surfactant concentration on the transport properties of bulk surfactant aqueous solutions, focusing on the anionic surfactant sodium dodecyl sulfate $[10,33]$. The surfactant self-diffusion and the thermal conductivity of bulk aqueous sodium dodecyl sulfate (SDS) solutions were computed at a range of concentrations at room and boiling temperatures. Additionally, MP2f [34], one of a new generation water potentials is assessed for its suitability in reproducing the transport and thermal properties of bulk water. The thermal conductivity of MP2f water model was found to be: 0.64 $\mathrm{W} /(\mathrm{m} \cdot \mathrm{K})$ at $298 \mathrm{~K}$ and $0.66 \mathrm{~W} /(\mathrm{m} \cdot \mathrm{K})$ at $373 \mathrm{~K}$, in much better agreement with the experimental values compared to both the rigid and the flexible TIP3P water model.

\subsection{Introduction}

Nucleate pool boiling is a very efficient and widely used cooling method with a wide variety of heat dissipation applications in industry and technology, e.g., in aircraft thermal management, electronics, and nanofluidics. A better understanding of the mechanisms and details of the multiscale phenomena involved in the process of boiling is needed to provide an insight into ways to control and enhance the boiling heat transfer. Additives have long been recognized and studied for the purposes of enhancing the boiling heat transfer [35-38] and heat transfer with surfactant additives in pool boiling is the topic of active research in thermal management [39-45], spray-cooling [46], micro and nano-fluidics [47-51]. Additives can enhance or diminish the effectiveness of boiling heat transfer depending on the chemistry or concentration of the additives $[35,37,38,44,48-50,52-56]$. On a macroscopic level, solution additives contribute to dynamic surface tension [36, 40,52, 57] and modify the surface wettability [58]. Since nucleate boiling is such ubiquitous thermal management method, there is a sustained interest in its enhancing, better understanding, and control [39, 42, 46, 47, 51, 59-61]. There have been a number of microscopic models attempting to describe nucleate boiling with additives [62-64] but there is still a need for better understanding on a molecular level. Recently, the effect of concentration, chemistry, temperature, and $\mathrm{pH}$ on thermal conductivity and viscosity of bulk aqueous surfactant solutions were studied experimentally by Zhou et al. [61]. It was observed that while the addition of any surfactant decreases the thermal conductivity of the solution, non-ionic surfactants reduce the thermal conductivity more than the ionic ones.

Our research interest is in the advancement of molecular-level understanding of the processes involved in the phenomenon of boiling with additives. The present effort attempts to address two specific aspects of the problem of interest: exploring the effect of adding the surfactant sodium dodecyl sulfate (SDS) on the thermal conductivity of the solutions at room and boiling temperature as well as the concentration dependence of the diffusion of SDS in water.

We carry out classical MD simulations [14], in which the individual atoms are approximated by spheres with van der Waals radii and partial charges, the intra-molecular interactions are approximated via (harmonic) potentials for chemical bonds, angles, and dihedrals, while the inter-molecular interactions are modeled by the long-range Lennard-Jones and Coulomb interactions.

At each simulation step, the Newtonian equations of motion are solved, i.e., numerically integrated for each atom in the potential field created by the rest of the atoms in the system. The force field (FF) parameters are usually optimized to correctly reproduce some experimental properties and/or are developed from $a b$ initio or density functional theory (DFT) calculations of

DISTRIBUTION STATEMENT A: Approved for public release. Distribution is unlimited. 
gas or condensed phase. There exist a large number of theoretical MD model potentials for water $[34,65-70]$ and each of them reproduces well only some of the water properties and only within certain thermodynamic conditions. The variety of water models can be broadly divided into: rigid, flexible, and polarizable.

Rigid models keep the bond lengths and angles at their equilibrium positions which significantly reduces the computational cost, especially for large systems. Additional computational savings come from the opportunity to use larger simulation steps. Since the timestep of the integration is chosen to be smaller than the period of the fastest motion in the system, usually, a timestep of $10^{-15} \mathrm{~s}$ is chosen to account for the fast motion of the hydrogen atoms in water molecules. If rigid bond models are used via algorithms like SHAKE [71] the timestep can be increased to $2 \times 10^{-15} \mathrm{~s}$.

Flexible water models allow the water molecule to deform, i.e., bond distances and the bond angle change in the course of the simulation, at the expense of increased computational cost. Polarizable force fields additionally allow for variable partial charges on the atoms of the water molecule in accordance to the changing environment and this is why they are exceptionally computationally costly. All force fields, therefore, account for the many-body effects to a varying degree since including the polarization effects is computationally demanding. Moreover, any classic force field description of quantum molecular bonds is inherently approximate [72]. On the other hand, there exist a variety of force fields that are parameterized for more or less specific system or class of systems in mind. For example, AMBER [73], CHARMM [74] and OPLS [75] force fields, to mention a few, are optimized for biomolecular simulations, while CLAYFF [76] was developed with the goal to be general enough and suitable for modeling aqueous solutions and interfaces of inorganic mineral materials. The complexity of the problem of choosing a water model and force field parameters to describe a molecular system is additionally increased by the specific suitability and compatibility of the water model with the model parameters to describe the rest of the simulated system. Therefore, the choice of a force field depends on its range of applicability and on the molecular system to be studied [67, 77].

Bulk liquid properties using rigid water models are extensively studied and available in the literature [34, 65, 78-80] [81]. Recently, the transport and thermal properties of well-established and widely used rigid and flexible water models were studied and compared. Mao and Zhang [77] evaluated the thermal conductivity, shear viscosity, and specific heat of seven rigid water models. The work of Sirk et al. [82] studied the thermal conductivity of common classic flexible water models, compared the computational approaches for evaluating thermal conductivity, and observed that although both rigid and flexible water models overestimate the thermal conductivity of neat water, the rigid water models provide values closer to experiment. It was hypothesized that the increased number of degrees of freedom of the flexible water models is responsible for the overestimation of the heat transfer. Although, the rigid water models are better suited for simulating heat transfer in bulk fluids, it was also suggested that the appropriateness of rigid vs. flexible water models at interfaces needs further investigation.

We report a computational study on the effect of surfactant concentration on the diffusion and thermal properties of aqueous SDS solutions at room and at boiling temperatures. We also assess the performance of a relatively new water model [34] derived from condensed phase $a b$ initio calculations, in simulating transport behavior of bulk water near boiling conditions along with room temperature. The model for SDS by Schweighofer et al. [83] was adopted which combines all-atom approach for the hydrophilic sulfate headgroup and united-atom course-grained approach for the hydrophobic tail of SDS. The diffusion coefficient of SDS and the thermal

DISTRIBUTION STATEMENT A: Approved for public release. Distribution is unlimited. 
conductivity of the aqueous surfactant solution is computed for different concentrations of SDS at room and boiling temperatures. The solvent model used is a standard TIP3P [80] water model. The results for the diffusion of SDS in water and the thermal conductivity of surfactant aqueous solutions are compared to experimental results. Further, we introduce the ab initio flexible water model developed by Akin-Ojo et al. [34] in 2008 using the relatively new adaptive force-matching method $[84,85]$ and compute the diffusion and thermal conductivity of bulk water predicted by this model.

\subsection{Transport Properties of Aqueous Surfactant Solutions at Room and Boiling Temperatures}

\subsubsection{Surfactant Model and Simulation Details}

In our work [10], we adopted the hybrid model of SDS [83, 86-88] which combines all-atom description of the hydrophilic sulfate headgroup of SDS with a united-atom approach to describe the hydrophobic aliphatic tail of SDS, bottom structure in Figure 3-1. The top structure of Figure 3-1 shows the atomic structure of the anionic surfactant SDS.

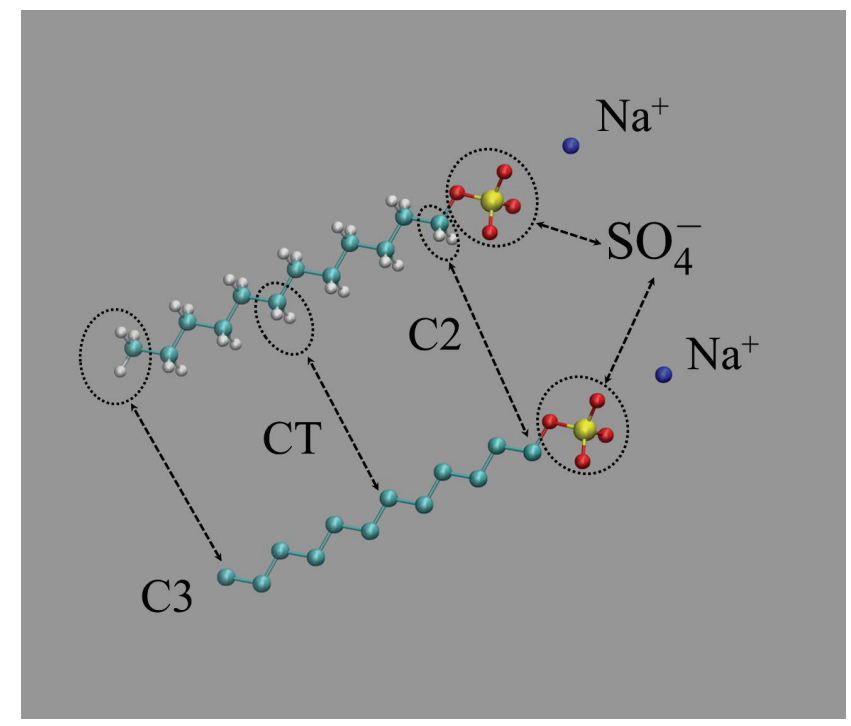

Figure 3-1: Molecular Models of SDS

((Top) All-atom model of sodium dodecyl sulfate. Color convention: red-oxygen, white-hydrogen, yellow - sulfur, cyan - carbon, dark blue - sodium ion. (Bottom) Hybrid all-atom united-atom model of $S D S$.)

An all-atom approach describes each atom with its own set of parameters and was used to describe the water molecules in this work. The united-atom approach is in a sense a coarse-graining approach in which a group of atoms is described as one united-atom with its own set of parameters. Only the hydrophobic tail is described by united-atom approach where the hydrogen atoms belonging to each carbon atom are grouped into a new united-atom. For example, the terminal $\mathrm{CH}_{3}$ group of SDS is represented by the $\mathrm{C} 3$ atom in united-atom approach. The $\mathrm{CH}_{2}$ groups are represented by a new atom type $\mathrm{CT}$, and the last $\mathrm{CH}_{2}$ group bonded to the hydrophilic head is designated as $\mathrm{C} 2$.

DISTRIBUTION STATEMENT A: Approved for public release. Distribution is unlimited. 


\subsubsection{Self-diffusion of SDS in Water}

The diffusion coefficient of a solute in water can be computed from the mean square displacement (MSD) of the center of mass of each solute molecule as follows:

$$
D=\frac{1}{6} \lim _{t \rightarrow \infty} \frac{d}{d t}\left\langle|\vec{r}(t)-\vec{r}(0)|^{2}\right\rangle
$$

where $\left\langle|\vec{r}(t)-\vec{r}(0)|^{2}\right\rangle$ is the mean square displacement averaged over all solute molecules and time origins.

In the simulations for obtaining the diffusion coefficient of SDS in water, the water potential used was TIP3P[80]. Two sets of simulations were performed: with rigid and flexible TIP3P, at room temperature, $298.15 \mathrm{~K}$, and at boiling temperature, $373 \mathrm{~K}$. The initial size of the simulation box was $100 \AA \times 100 \AA \times 100 \AA$, containing approx. $3.3 \times 10^{3}$ water molecules. The initial simulation boxes were built using Packmol [89] and were further minimized and equilibrated at the respective temperature. Periodic boundary conditions were imposed in all three directions to simulate bulk properties and particle-particle particle-mesh (PPPM) method as implemented in LAMMPS [90, 91], also related to Ewald summation [14, 92], was applied to correctly account for the long-range electrostatic interactions.

All simulations were carried out using the Large-scale Atomic/Molecular Massively Parallel Simulator, LAMMPS [91]. The input files for LAMMPS were prepared using VMD [93]. After a standard procedure of minimization and equilibration, we performed 2-ns equilibrium MD runs at constant volume and temperature of the system at $298.15 \mathrm{~K}$ and $373 \mathrm{~K}$, respectively, in order to obtain the self-diffusion of SDS in water.

\subsubsection{Thermal Conductivity}

Thermal conductivity can be computed from equilibrium MD simulations using the GreenKubo formula [94] or by non-equilibrium MD (NEMD) simulations by imposing a known heat flux in the system. We computed the thermal conductivity by carrying out NEMD simulations where heat flux is introduced in the system which leads to establishing a temperature gradient across the system as proposed by Müller-Plathe [95]. We used the Müller-Plathe method of imposing heat flux [96] which involves swapping the kinetic energy of the hottest particle in a predefined cold region of the system with the kinetic energy of the coldest particle in the predefined hot region of the system thus conserving the total energy of the system while inducing heat flux from the hot region (heat source) to the cold region (heat sink). In response to the heat flux through the system, a temperature gradient is established. After equilibrating the simulation systems at the respective temperature in NVT ensemble, NEMD runs on NVE ensemble were performed, during which a steady temperature gradient in z-direction was established. From the last 700 ps of the simulations, the temperature profiles were recovered and the temperature gradient, $d T / d z$, was computed from linear fits of the temperature profiles. The temperature gradient is induced by the heat flux, $J$, imposed on the system and the coefficient of proportionality is the thermal conductivity, $\lambda$, of the material: $J=-\lambda d T / d z$.

\subsubsection{Diffusion of SDS in Water: Results and Discussion}

When Sodium Dodecyl Sulfate $\left(\mathrm{NaC}_{12} \mathrm{H}_{25} \mathrm{SO}_{4}\right)$ dissolves in water, the ionic bond between $\mathrm{Na}^{+}$and $\mathrm{DS}^{-}\left(\mathrm{C}_{12} \mathrm{H}_{25} \mathrm{SO}_{4}{ }^{-}\right)$breaks and the sodium counterions dissociate. The degree of dissociation and the fraction of sodium ions that is associated in the first and second solvation shells can be determined from the sodium-sulfur radial distribution function [97]. For the purposes 
of computing the diffusion of SDS in water and comparing to the experimental values [98], and since the light sodium ion is far more mobile than the $\mathrm{DS}^{-}$anion, hereafter we consider the diffusion of the anion DS $^{-}$and refer to it as SDS. The diffusion coefficient of SDS was computed from the MSD of the center of mass of each molecule, according to Eq.(3-1). The computed values at 298.15 $\mathrm{K}$ are shown in Table 3-1 and compared with the experimental values from [98].

Table 3-1: Diffusion Coefficient $D_{s}$ of Aqueous SDS Solutions at 298.15 K

(The values of the diffusion coefficient $D_{\text {s }}$ are listed in $10^{-6} \mathrm{~cm}^{2} / \mathrm{s}$ and are computed for a range of concentrations. The experimental results (\$) are taken from Ref. [98].)

\begin{tabular}{llrr}
\hline $\mathrm{C}(\mathrm{mol} / \mathrm{L})$ & $\operatorname{Exp}^{\S}$ & TIP3P(f) & TIP3P(r) \\
\hline 0.0016 & - & 0.51 & 8.18 \\
0.0050 & - & 7.16 & 4.64 \\
0.0082 & - & 5.89 & 4.81 \\
0.0100 & 1.76 & 2.87 & 6.12 \\
0.0164 & 3.00 & 8.47 & 12.34 \\
0.0500 & 4.03 & 6.32 & 13.18 \\
0.1250 & 4.53 & 3.09 & 4.23 \\
\hline \hline
\end{tabular}

It should be noted that the computation of the MSD at low surfactant concentrations is inherently less accurate than at larger concentrations. Surfactant concentration of $0.0016 \mathrm{~mol} / \mathrm{L}$ corresponds to a single SDS molecule in our simulation box whereas a surfactant concentration of $0.125 \mathrm{~mol} / \mathrm{L}$ corresponds to $75 \mathrm{SDS}$ molecules in the simulation box which provide better statistics for MSD. From Table 3-1, it is clear that the computed values of the diffusion coefficient of SDS in water are of the correct order of magnitude. At higher concentrations, where we expect better accuracy, the rigid TIP3P water potentials systematically predicts larger values than the flexible one. A similar trend is also observed at boiling temperature, as can be seen from Figure 3-2, in which the diffusion coefficient of SDS computed at $373 \mathrm{~K}$ is plotted as a function of the surfactant concentration. Again, the diffusion coefficients of SDS in rigid TIP3P water are always larger than the values predicted by using flexible water potential.

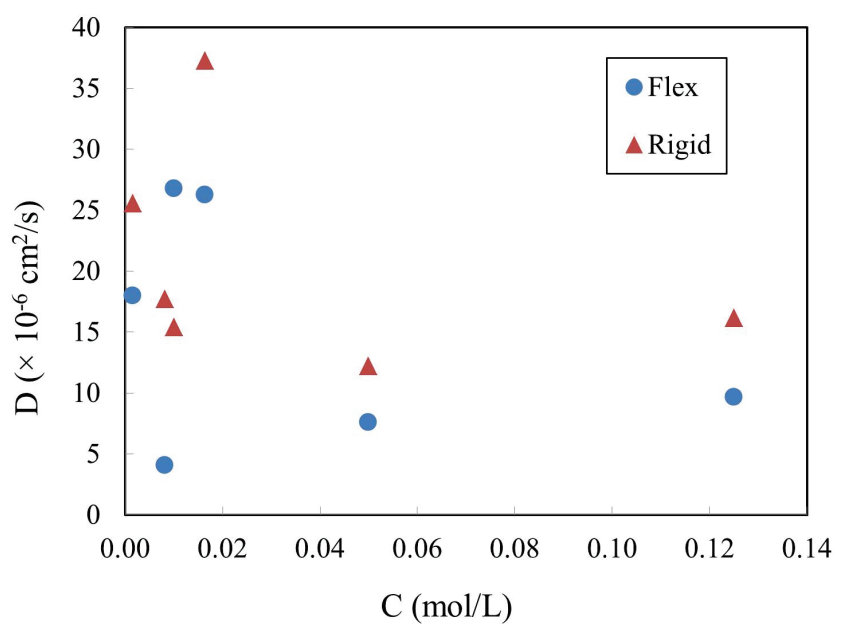

Figure 3-2: Diffusion Coefficient of SDS at Different Surfactant Concentrations at $373 \mathrm{~K}$ (The values were computed with the flexible TIP3P (Flex) and the rigid TIP3P (Rigid) water potential.)

11

DISTRIBUTION STATEMENT A: Approved for public release. Distribution is unlimited. 
The calculations from simulations with the rigid water model seem to systematically overestimate the diffusion coefficient of SDS at higher surfactant concentrations. At lower surfactant concentrations, the accuracy of the simulated results is lower, probably, due to insufficient statistics and simulations of longer duration and/or larger scale can improve the accuracy of the estimates.

\subsubsection{Thermal Conductivity of Aqueous SDS Solutions: Results and Discussion}

The thermal conductivities of aqueous SDS solutions at a range of surfactant concentrations were computed by Müller-Plathe's method $[95,96]$ from NEMD simulations. The values of the thermal conductivity of aqueous SDS solutions, computed with flexible and rigid TIP3P water model at room and boiling temperature and different surfactant concentrations are listed in Table $3-2$.

Table 3-2: Thermal Conductivity $\lambda\left[\frac{W}{m \cdot K}\right]$ of Aqueous SDS Solutions at 298.15 K and $373 \mathrm{~K}$

\begin{tabular}{lccccc}
\hline \hline C (mol/L) & TIP3P(f) & TIP3P(r) & TIP3P(f) & TIP3P(r) \\
\hline 0.0 & \multicolumn{2}{c}{$298.15 \mathrm{~K}$} & & $373 \mathrm{~K}$ & \\
0.0016 & 0.96 & 0.82 & 1.00 & 0.68 \\
0.0082 & 0.91 & 0.80 & 0.96 & 0.83 \\
0.0164 & 0.92 & 0.72 & 0.95 & 0.76 \\
0.0500 & 0.91 & 0.80 & 0.96 & 0.84 \\
0.1250 & 0.90 & 0.80 & 0.94 & 0.83 \\
\hline \hline
\end{tabular}

Recently, an experimental investigation of the effects of the surfactant concentration, temperature, and $\mathrm{pH}$ on the thermal conductivity of aqueous solutions of non-ionic, cationic, and anionic surfactants, was reported [61]. Compared to the reported values in Ref. [61], both water models lead to higher than experimental thermal conductivity of the aqueous SDS solutions. As in the case of pure water, the flexible water potential leads to an overestimate of the thermal conductivity of the solution. Both models correctly predict the increase of the thermal conductivity with increase of temperature.

In Ref. [61] it was experimentally observed that the room temperature thermal conductivity ratio $\left(\frac{\lambda}{\lambda_{\text {water }}}\right)$ of the ionic surfactants SDS and SDBS increases at lower concentrations, approx. $0.1 \mathrm{wt} \%$ for SDS, and that with increasing the concentration the thermal conductivity ratio gradually decreases. At lower concentrations, it was observed that the thermal conductivity of the ionic surfactant solution was larger than the thermal conductivity of neat water. Our simulations did not reproduce the enhancement of the thermal conductivity of the surfactant solutions at low concentrations but did reproduce the experimentally observed slow decrease trend at concentrations higher than the critical micelle concentration (CMC). Clearly, further simulations on a much larger scale are needed to better understand the thermal properties of low concentration surfactant solutions, as well as to explore the effects of the chosen surfactant and solvent models, simulation length and system size on the computational results. Studies with increasingly large simulation systems can be the subject of future works. 


\subsection{MP2f Water Model and Simulation Details}

With respect to reproducing the transport properties of water, and more specifically, the selfdiffusion of water, the modified TIP3P [80] water potential used in this work is the most commonly used and reliable force field as it was optimized to better reproduce not only the experimental water density, heat of vaporization, and dielectric constant but also the experimental bulk diffusion coefficient of water.

The flexible force field MP2f [34] was developed by the relatively new adaptive force matching method from condensed phase QM/MM calculations. The force matching (FM) method was first proposed in 1994 [84]. The FM method optimizes the force field parameters not to best reproduce any experimental bulk water properties, as is the case in the empirical force fields, nor to best fit single point energies from ab initio gas phase calculations, as is the case in the usual $a b$ initio force fields, but, instead, to reproduce $a b$ initio forces derived from density functional theory (DFT) calculations in the condensed phase [99]. The adaptive force matching (AFM) method is an improvement upon the original FM schemes in that it uses quantum-mechanics/molecularmechanics $(\mathrm{QM} / \mathrm{MM})$ calculations and avoids the need for expensive condensed phase electronic structure calculations [34]. Therefore, one major appeal of the AFM method lies in the fact that the force field parameters are derived from condensed phase calculations as opposed to the usual gas phase calculations, since those force field parameters are intended to be used to simulate bulk liquid water.

In order to investigate the transport properties of the MP2f water model, we computed the diffusion constant of the model and the thermal conductivity of water at room temperature 298.15 $\mathrm{K}$ and boiling temperature $373 \mathrm{~K}$.

\subsubsection{Self-diffusion of Bulk Water}

We performed 2-ns equilibrium MD runs to compute the diffusivity of water at two temperatures, $298.15 \mathrm{~K}$ and $373 \mathrm{~K}$, using MP2f water potential. The simulation step was $0.001 \mathrm{ps}$. The simulation runs were performed at constant volume and temperature using Nose-Hoover thermostat as implemented in LAMMPS [91]. Snapshots of the atomic positions and velocities were output every 100 simulation steps or every $0.1 \mathrm{ps}$. The self-diffusion coefficient of water is computed from the mean square displacement (MSD) of the center of mass of each water molecule or, to a very good approximation, the position of the oxygen atom in each water molecule, according to Eq. (3.1).

As it is well known that the computed values of the diffusion coefficient depend on the size of the simulation box [100-102] when periodic boundary conditions are applied, we set up a series of six simulation systems, containing 128, 256, 512, 1024, 2048, and 4096 water molecules, at normal water density of $33.0 \mathrm{~nm}^{-3}$. If $L$ is the length of the periodic water box, and $D_{L}$ is the computed diffusion coefficient in the same periodic water box, then the dependence of $D_{L}$ on the length $L$ is given by:

$D_{L}=D_{0}-\frac{2.837 k_{B} T}{6 \pi \eta L}$

In Eq. (3-2) $\eta$ is the shear viscosity of water. Therefore, we can extrapolate the size-independent diffusion coefficient $D_{0}$ and the viscosity of water from the series of simulations of increasing simulation box size. 


\subsubsection{Self-diffusion and Thermal Conductivity of MP2f Water Model: Results and Discussion}

The values of the self-diffusion coefficient of water obtained from simulations in different size periodic simulation boxes were fitted with a linear function of $1 / L$ as shown in Figure 3-2. From these fits, the size-independent values of the diffusion coefficients in infinitely large bulk system were extrapolated. The fitting procedure provides an estimate of the viscosity of the water model as well. The extrapolated size-independent values of the diffusion coefficient of MP $2 \mathrm{f}$ water model are shown in Table 3-3.

Table 3-3: Size-independent Diffusion Coefficient $\left(D_{0}\right)$ and Viscosity $(\eta)$ of MP2f Water Model at 298.15 $\mathrm{K}$ and $373 \mathrm{~K}$

(For comparison, estimates of the size-independent diffusivity of other water models and experimental values are provided. ${ }^{(a)}$ [103], ${ }^{(b)}$ [104], ${ }^{(c)}$ [101], ${ }^{(d)}$ [102], ${ }^{(e)}$ [77], ${ }^{(f)}$ [77]-this value was calculated at $363 \mathrm{~K}$.)

\begin{tabular}{lllll}
\hline \hline Model & $\mathrm{D}_{0}\left[\times 10^{-5} \mathrm{~cm}^{2} / \mathrm{s}\right]$ & \multicolumn{3}{l}{$\eta\left[\times 10^{-4} \mathrm{~kg} /(\mathrm{m} \cdot \mathrm{s})\right]$} \\
\hline MP2f & $298.15 \mathrm{~K}$ & $373 \mathrm{~K}$ & $298.15 \mathrm{~K}$ & $373 \mathrm{~K}$ \\
IP3P & 4.06 & 9.95 & 3.24 & 3.84 \\
SPC/E & $6.05^{(\mathrm{c})}$ & - & $3.1^{(\mathrm{e})}$ & $2.17^{(\mathrm{f})}$ \\
TIP4P/2005 & $2.97^{(\mathrm{d})}$ & - & $6.4^{(\mathrm{d})}$ & - \\
Exp. & $2.49^{(\mathrm{d})}$ & - & $8.3^{(\mathrm{d})}$ & - \\
\hline \hline
\end{tabular}

In the table, we also provide literature values obtained by the same extrapolation technique. The three literature water models are rigid. Our estimate of the viscosity of the MP2 $\mathrm{f}$ water models is: $3.24 \times 10^{-4} \mathrm{~kg} /(\mathrm{m} \cdot \mathrm{s})$ at $298.15 \mathrm{~K}$ and $3.84 \times 10^{-4} \mathrm{~kg} /(\mathrm{m} \cdot \mathrm{s})$ at $373 \mathrm{~K}$. From Table $3-3$, it can be observed that MP2 $\mathrm{f}$ water potential is somewhat better than rigid TIP3P with respect to the predicted value of the size-independent diffusion coefficient but SPC/E [105] and TIP4P/2005 [106] predict size-independent values of diffusion coefficient and viscosity of water in better agreement with experiment. Table 3-3 also lists recently reported values for the shear viscosities of a number of rigid water models computed using the Green-Kubo method [77].

The values of the computed thermal conductivity of water are shown in Table 3-4. It was reported that the computed value of the water thermal conductivity was found to be independent on the size of the simulation box; therefore, we performed all simulations in a periodic box containing $4.096 \times 10^{3}$ water molecules. The results in Table 3-4 confirm the observation from [82] that the flexible water models tend to overestimate the thermal conductivity of water, TIP3P(f) vs. TIP3P(r). Our results further show that the flexible MP2f water model performs exceptionally well in reproducing thermal conductivity of water at room and boiling temperature, compared to both the rigid and flexible TIP3P. 
Table 3-4: Computed Thermal Conductivity $\lambda$ in $W /(m \cdot K)$ of Neat Water at Room and Boiling Temperature

\begin{tabular}{lll} 
& (The experimental values are from: ${ }^{(a)}[107]$ and $\left.^{(b)}[108].\right)$ \\
\hline \hline & $298.15 \mathrm{~K}$ & $373 \mathrm{~K}$ \\
\hline TIP3P(f) & 0.96 & 1.00 \\
MP2f & 0.64 & 0.66 \\
TIP3p(r) & 0.82 & 0.68 \\
Exp. & $0.607^{(\mathrm{a})}$ & $0.6723^{(\mathrm{b})}$ \\
\hline \hline
\end{tabular}

In conclusion, the flexible MP2f water potential, derived by AFM method from condensed phase QM/MM calculations is shown to perform reasonably well in reproducing the diffusion coefficient of water and very well in reproducing the thermal conductivity of water at both room and boiling temperature.

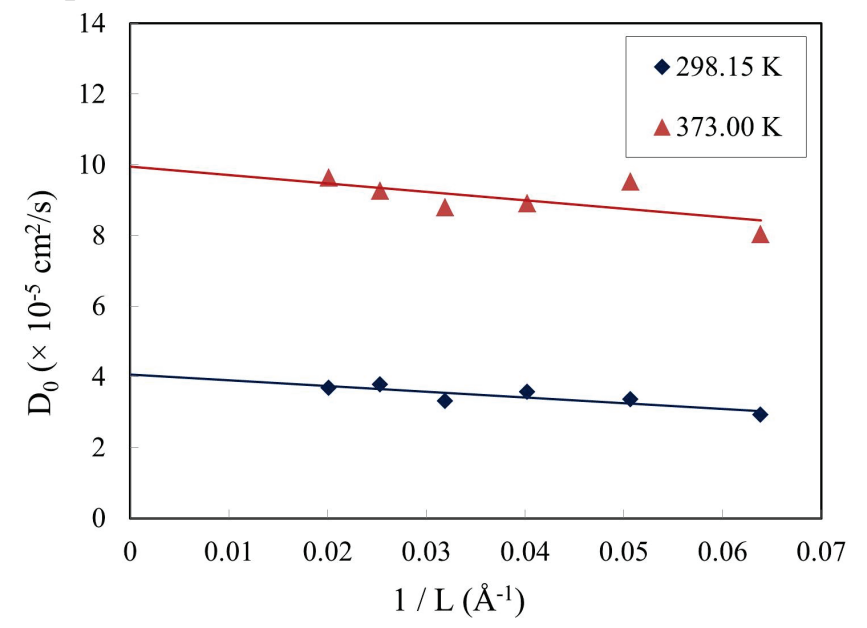

Figure 3-3: Diffusion Coefficient as a Function of the Inverse Length of the Periodic Simulation Box

(From a linear fit of the data, the size-independent diffusion coefficients for MP $2 f$ water model were extrapolated to be $4.06 \times 10^{-5} \mathrm{~cm}^{2} / \mathrm{s}$ at $298.15 \mathrm{~K}$ and $9.95 \times 10^{-5} \mathrm{~cm}^{2} / \mathrm{s}$ at $373 \mathrm{~K}$.)

\subsection{Conclusions}

We performed an extensive series of MD simulations computing the transport properties of bulk aqueous surfactant solutions at room and boiling temperatures for a range of surfactant concentrations. The effect of the water model on reproducing the surfactant diffusivity and the thermal conductivity of aqueous SDS solutions was investigated by using flexible and rigid TIP3P water. It was observed that at higher surfactant concentrations, the rigid water model overestimates the values for surfactant diffusion. At lower concentrations, the results were less reliable due to insufficient statistics and further simulations with larger systems are required to improve the accuracy of the estimates. It was recently observed in the case of pure water [82], that the flexible water models are not only more computationally costly but they tend to overestimate the thermal conductivity of neat water. Our results for the thermal conductivity of aqueous SDS solutions confirm this but also show that when the thermal conductivity enhancement of the solution with respect to pure water is concerned, the flexible water model reproduces the experimental trend more reliably, at least at concentrations higher than CMC. Future larger scale MD studies are 
needed to further explore and better understand the transport properties of surfactant solutions at low concentrations.

We also studied the ability of MP2f, an ab initio water potential derived from condensed phase calculations, to reproduce the diffusion coefficient and thermal conductivity of water at room and boiling temperatures. The size-independent diffusion coefficient of water was derived from a series of MD simulations with increasing size of the simulation system. The bulk water self-diffusion coefficient was estimated to be $4.05 \times 10^{-5} \mathrm{~cm}^{2} / \mathrm{s}$ at $298 \mathrm{~K}$ and $9.95 \times 10^{-5} \mathrm{~cm}^{2} / \mathrm{s}$ at 373 $\mathrm{K}$. The computed values of the thermal conductivity were: $0.64 \mathrm{~W} /(\mathrm{m} \cdot \mathrm{K})$ at $298 \mathrm{~K}$ and 0.66 $\mathrm{W} /(\mathrm{m} \cdot \mathrm{K})$ at $373 \mathrm{~K}$, in much better agreement with the experimental values compared to both the rigid and the flexible TIP3P. 


\section{SOLVENT EFFECTS IN THE THERMAL DECOMPOSITION REACTION OF AMMONIUM CARBAMATE}

The endothermic decomposition of ammonium carbamate has been proposed as a novel heat sink mechanism for aircraft thermal management [109]. The products of the reversible decomposition are carbon dioxide and ammonia which need to be efficiently removed from the carrier fluid in order to better control the reaction and thus the heat transfer. MD simulations can provide insight into the transport properties of carbon dioxide and ammonia in the carrier fluid. In this work, extensive classical non-reactive molecular dynamics simulations were carried out to explore the solvent effects in the thermal decomposition reaction of ammonium carbamate by studying the temperature and concentration dependence of relative solubility and diffusivity of carbon dioxide and ammonia in water, ethylene glycol, and their mixtures at standard temperature and pressure and at the elevated temperature of the thermal decomposition reaction of ammonium carbamate. This comparative study shows that ammonia is more soluble than carbon dioxide in either water or ethylene glycol and that both carbon dioxide and ammonia are more soluble in ethylene glycol than in water. Our simulations of water - ethylene glycol mixtures show that increasing the molar fraction of ethylene glycol leads to increased solubility of carbon dioxide and ammonia in the mixture. Even though this is a non-reactive study, it is able to capture the general solubility trends. Accounting for the reactions of carbon dioxide and ammonia in the solution would further amplify the observed trends by amplifying the retaining of $\mathrm{CO}_{2}$ and $\mathrm{NH}_{3}$ in the solution. Our work [110] presents a low-cost computational procedure for relative solubility evaluation that can be used in a broader engineering design context.

\subsection{Introduction}

The developments in modern aircraft and electronics pose ever increasing demands on thermal management applications and have prompted a heightened interest in the search of application-tailored phase change mechanisms and materials as heat sinks. Endothermic reactions represent novel opportunities for heat sinks and the implications and specific challenges they also present need further exploration. Ammonium carbamate has emerged as an expendable high energy density thermal management material in aircraft thermal management [111-114]. The reaction kinetics of the ammonium carbamate decomposition has proven to be elusive [115] and complex [116]. A Raman spectroscopy study on ammonium carbonate, ammonium bicarbonate, ammonium carbamate, and ammonium chloride with sodium carbonate allowed for an estimate of the equilibrium constants and thermodynamic parameters and showed the carbamic acid to be unstable with respect to decomposition into carbon dioxide and ammonia [117]. The relative concentration of carbonate decreased with increase of temperature compared to the concentration of bicarbonate and carbamate. The relative stabilities and optimal geometries of $\mathrm{H}_{2} \mathrm{NCOO}^{-}$, $\mathrm{H}_{2} \mathrm{NCOOH}^{+}, \mathrm{H}_{3} \mathrm{NCOO}^{-}$have been evaluated using ab initio restricted Hartree-Fock calculations

and it has been established that the zwitterion was the most probable intermediate for the decomposition of carbamic acid into ammonia and carbon dioxide, and that the zwitterion is an unstable species [117].

There has been a significant interest and focus on the $\mathrm{CO}_{2}+\mathrm{NH}_{3}$ reaction for the purposes of post-combustion carbon dioxide capture and storage and ammonium carbamate is studied as a product or intermediate in the $\mathrm{CO}_{2}$ capture in ammonia. All these studies help to provide better understanding of the reverse reaction, the decomposition of ammonium carbamate as well. Ammonia scrubbing has been proposed and explored for $\mathrm{CO}_{2}$ removal from flue gas [118-120]. A review paper by White et al. [121] discussed the methods being considered and employed for

DISTRIBUTION STATEMENT A: Approved for public release. Distribution is unlimited. 
separation, capture, and sequestration of $\mathrm{CO}_{2}$, including in large geological formations, see also Benson and Cole[122]. Dissolution of carbon dioxide in brine is studied in connection with $\mathrm{CO}_{2}$ sequestration in geological formations [123]. There is a large number of experimental [124-126] and theoretical investigations of carbon dioxide capture in ammonia. The reaction kinetics of absorption of $\mathrm{CO}_{2}$ in aqueous ammonia solutions has been studied at a range of temperatures [126131]. Jeon et al. [131] have experimentally shown that hydroxyl additives (including ethylene glycol) enhance the absorption rate of $\mathrm{CO}_{2}$ in aqueous ammonia as well as reduce the volatility of ammonia. Zwitter-ion mechanism $[132,133]$ was proposed to describe the reaction $\mathrm{CO}_{2}+\mathrm{NH}_{3}$ as a two-stage reaction. Choi et al. [134] experimentally investigated the role of temperature in the aqueous reaction $\mathrm{CO}_{2}+\mathrm{NH}_{3}$ and observed that at $278 \mathrm{~K}$, the dominant reaction becomes bicarbonation, while at $298 \mathrm{~K}$ the dominant reaction is carbamation.

A review by Zhao et al. [135] presents an overview of the current understanding and the fundamental research in post-combustion capture of $\mathrm{CO}_{2}$ in aqueous ammonia. Other solvents and mixtures are being considered for more efficient $\mathrm{CO}_{2}$ capture. For example, Puxty et al. [136] experimentally compared the rate of $\mathrm{CO}_{2}$ absorption in ammonia and monoethanolamine. More recently, ionic liquids are being explored as promising candidates for very efficient postcombustion $\mathrm{CO}_{2}$ capture [120,137-139] since the diffusivities of carbon dioxide in ionic liquids are one to two orders of magnitude less than in other liquid solvents [140]. The interest in carbon dioxide solubility in aqueous and non-aqueous solutions and mixtures is driven not only by the need to identify those solvents and mixtures providing enhanced capture but also by the need to consequently strip the captured gas in an efficient and controlled manner [141, 142].

The reaction of carbon dioxide and ammonia in solutions has proven to be a complex multistep process and the same is true for the reverse reaction of ammonium carbamate decomposition. Therefore, the goal of this work was to study the solvent effects in the thermal decomposition reaction of ammonium carbamate by employing classical non-reactive molecular dynamics simulations to quantify the solubility of carbon dioxide and ammonia in ethylene glycol, water, and their mixtures, the effects of temperature and carrier fluid composition, and to assess the suitability of ethylene glycol as a carrier fluid for the reaction of interest. This work focuses on an idealized and simplified situation as a first-approximation approach and further complexity can be added in future studies. Our approach does not take into account any reaction of the product gases and the carrier fluid but focuses, instead, at the solubility and diffusivity of the gases. The salt effects were also not the topic of this work as the experimental setup of the reactive heat exchanger does not involve additives but the effect of contaminants should also be taken into account in the design of the experimental setup.

\subsection{Simulation Method and Models}

In this study, we use classical MD simulations [14], in which each atom is modeled by the position of its nucleus, carries a partial electric charge, and its motion occurs in the potential field created by the rest of the atoms in the system. The interactions within a molecular system are approximated by intramolecular (bonded) interactions: bond stretching, bond bending, and torsions, and pairwise intermolecular (non-bonded) interactions: Lennard-Jones $\mathrm{R}^{-12}$ and $\mathrm{R}^{-6}$ interactions, and Coulomb interactions between any two charged particles. The set of parameters are usually called force field parameters and are either fitted to correctly reproduce some experimental properties (e.g., density and/or self-diffusion coefficient) or are derived from $a b$ initio or density functional theory calculations of gas or condensed phase of the material being modeled. 


\subsubsection{Solvents: Ethylene Glycol and Water}

Figure 4-1 shows the molecular structure of ethylene glycol (EG) in a ball-and-stick representation as well as the atomic labels that were used in this work to specify atom types. The carbon atoms are shown in cyan and labeled CT, the oxygen atoms are shown in red and labeled $\mathrm{OH}$, and all hydrogen atoms are white and labeled $\mathrm{OH}$ when they are in a hydroxyl group or $\mathrm{HC}$ when they are bonded to a carbon atom.

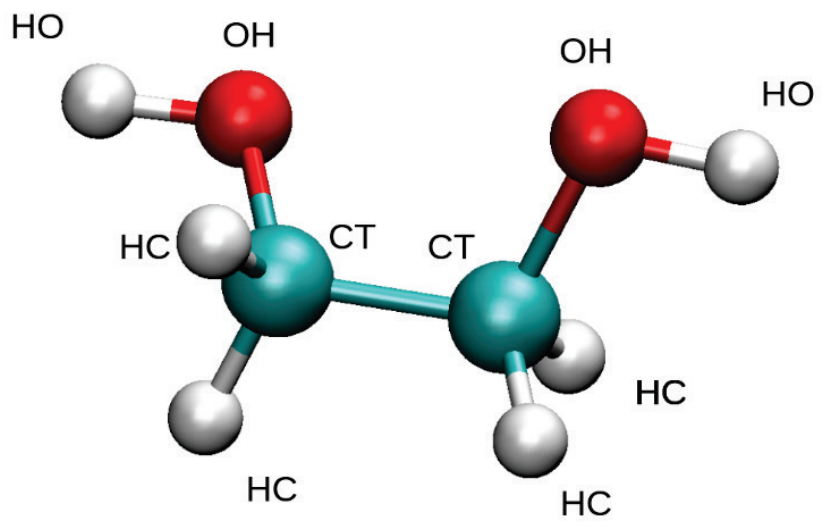

Figure 4-1. Atomic Structure of a Single Molecule of Ethylene Glycol

The performance of four different force fields with respect to predicting the structure of liquid ethylene glycol was evaluated by Saiz et al. [143]. In the study, the Lennard-Jones parameters for all models were the same while the partial charges were different and the results showed that the OPLS-based force fields reproduce more accurately the self-diffusion of ethylene glycol. Most recently, Szczerba et al. [144] used AMBER force field to describe ethylene glycol intercalation in smectites and flexible SPC force field for water. An earlier work by Kaiser et al. [145] used OPLS-AA force field and these are the force field parameters adopted in this work, namely, OPLS/AMBER force field [146-149]. List of all parameters used to model ethylene glycol in this work is provided in Table 4-1. The bond stretch energy for each pair of bonded atoms is:

$E_{\text {bond }}=k_{r}\left(r-r_{e q}\right)^{2}$

The bond bend (angle) energy is:

$E_{\text {angle }}=k_{\theta}\left(\theta-\theta_{e q}\right)^{2}$

And the torsion energy for each dihedral angle $\varphi$ is:

$E_{\text {tor }}=\frac{V_{1}}{2}(1+\cos \varphi)+\frac{V_{2}}{2}(1-\cos 2 \varphi)+\frac{V_{3}}{2}(1+\cos 3 \varphi)+\frac{V_{4}}{2}(1-\cos 4 \varphi)$

where $\varphi$ are the dihedral angles in the ethylene glycol molecule listed in Table 4-1. The nonbonded interactions are described by Lennard-Jones and Coulomb interaction energy as follows:

$E_{\text {non-bond }}=\sum_{i, j} 4 \varepsilon_{i j}\left(\left(\frac{\sigma_{i j}}{r_{i j}}\right)^{12}-\left(\frac{\sigma_{i j}}{r_{i j}}\right)^{6}\right)-\sum_{i<j} \frac{q_{i} q_{j}}{4 \pi \epsilon_{0} r_{i j}}$

where the mixing of the Lennard-Jones parameters is geometric: $\sigma_{i j}=\sqrt{\sigma_{i} \sigma_{j}}$ and $\varepsilon_{i j}=\sqrt{\varepsilon_{i} \varepsilon_{j}}$, and $\epsilon_{0}=8.9 \times 10^{-12} \mathrm{~F} / \mathrm{m}$ is the permittivity of free space. 


\section{Table 4-1. Values of the Force Field Parameters for Ethylene Glycol}

(The OPLS-AA force field values used in this work are from [145, 146]. The units are as follows: $\mathrm{m}$ [a.u], $\left.\mathrm{q}[\mathrm{e}], \sigma[\AA], \varepsilon[\mathrm{kcal} / \mathrm{mol}], k_{r}\left[\mathrm{kcal} /\left(\mathrm{mol} \cdot \AA^{2}\right)\right], r_{e q}[\AA], k_{\theta}\left[\mathrm{kcal} /\left(\mathrm{mol} \cdot \mathrm{rad}^{2}\right)\right], \boldsymbol{\theta}\left[{ }^{\circ}\right], V_{i}[\mathrm{kcal} / \mathrm{mol}], i=1, \ldots 4.\right)$

\begin{tabular}{|c|c|c|c|c|}
\hline Atoms & $\mathrm{m}$ & $\mathrm{q}$ & $\sigma$ & $\varepsilon$ \\
\hline $\mathrm{CT}$ & 12.01 & 0.145 & 3.500 & 0.066 \\
\hline $\mathrm{HC}$ & 1.008 & 0.060 & 2.500 & 0.030 \\
\hline $\mathrm{OH}$ & 15.999 & -0.700 & 3.070 & 0.170 \\
\hline $\mathrm{HO}$ & 1.006 & 0.435 & 0.000 & 0.000 \\
\hline Bonds & $\mathrm{k}_{r}$ & $\mathrm{r}_{\mathrm{eq}}$ & & \\
\hline $\mathrm{HO}-\mathrm{OH}$ & 553.0 & 0.945 & & \\
\hline $\mathrm{CT}-\mathrm{CT}$ & 268.0 & 1.526 & & \\
\hline $\mathrm{CT}-\mathrm{HC}$ & 340.0 & 1.090 & & \\
\hline $\mathrm{CT}-\mathrm{OH}$ & 320.0 & 1.410 & & \\
\hline Angles & $k_{\theta}$ & $\theta$ & & \\
\hline $\mathrm{CT}-\mathrm{OH}-\mathrm{HO}$ & 55.0 & 108.5 & & \\
\hline $\mathrm{CT}-\mathrm{CT}-\mathrm{HC}$ & 37.5 & 110.7 & & \\
\hline $\mathrm{HC}-\mathrm{CT}-\mathrm{HC}$ & 33.0 & 107.8 & & \\
\hline $\mathrm{CT}-\mathrm{CT}-\mathrm{OH}$ & 50.0 & 109.5 & & \\
\hline $\mathrm{HC}-\mathrm{CT}-\mathrm{OH}$ & 35.0 & 109.5 & & \\
\hline Dihedral angles & $\mathrm{V}_{1}$ & $\mathrm{~V}_{2}$ & $\mathrm{~V}_{3}$ & $\mathrm{~V}_{4}$ \\
\hline $\mathrm{OH}-\mathrm{CT}-\mathrm{CT}-\mathrm{OH}$ & 9.066 & 0.0 & 0.0 & 0.0 \\
\hline $\mathrm{HC}-\mathrm{CT}-\mathrm{CT}-\mathrm{OH}$ & 0.0 & 0.0 & 0.468 & 0.0 \\
\hline $\mathrm{HC}-\mathrm{CT}-\mathrm{CT}-\mathrm{HC}$ & 0.0 & 0.0 & 0.318 & 0.0 \\
\hline $\mathrm{CT}-\mathrm{CT}-\mathrm{OH}-\mathrm{HO}$ & 2.674 & -2.883 & 1.026 & 0.0 \\
\hline $\mathrm{HC}-\mathrm{CT}-\mathrm{OH}-\mathrm{HO}$ & 0.0 & 0.0 & 0.450 & 0.0 \\
\hline
\end{tabular}

The water model used in this work was the rigid extended single point charge (SPC/E) pair potential [105] which reproduces very well the density and diffusion constant of water at $300 \mathrm{~K}$ $[79,105]$. The bonds and the angle within the water molecules are kept rigid by employing the SHAKE algorithm [71] which does not compromise the transport properties of the simulated water as recent studies have shown that rigid models better reproduce the experimental diffusivity and thermal conductivity of water for the majority of the most popular water models $[10,82]$.

\subsubsection{Decomposition Products: Carbon Dioxide and Ammonia}

The molecular structure of a carbon dioxide molecule is shown on the left of Figure 4-2. The usual color convention is followed in which the carbon atom is shown in cyan and the oxygen atoms are red. Moultos et al. [150] computed the diffusivity of carbon dioxide in water at different temperatures and pressure. In a recent work, Garcia-Ratés et al. [151] used MD simulations to study the diffusion coefficients, ionic conductivity, and rotational relaxation of $\mathrm{CO}_{2}$ in aqueous ionic solutions (brines). More recently, Criscenti and Cygan [152] used the flexible force field to compare the cation solvation in water and supercritical carbon dioxide. The solubility of carbon dioxide in 1-ethyl-3-methylimidazolium Bis(trifluoromethylsulfonyl)imide was studied by means

DISTRIBUTION STATEMENT A: Approved for public release. Distribution is unlimited. 
of molecular dynamics simulations [153]. In this work, we use the fully flexible force field developed by Cygan et al. [154] and based on the original carbon dioxide force field by Harris and Yung [155]. The bond parameters are: $k_{r}=1008.963 \mathrm{kcal} /\left(\mathrm{mol} \cdot \AA^{2}\right)$ and $r_{\mathrm{eq}}=1.162 \AA$ and the angle parameters are: $k_{\theta}=108.0067 \mathrm{kcal} /\left(\mathrm{mol} \cdot \mathrm{rad}^{2}\right) \theta_{e q}=180^{\circ}$. The non-bonded force field parameters for carbon dioxide used in this work are listed in Table 4-2.
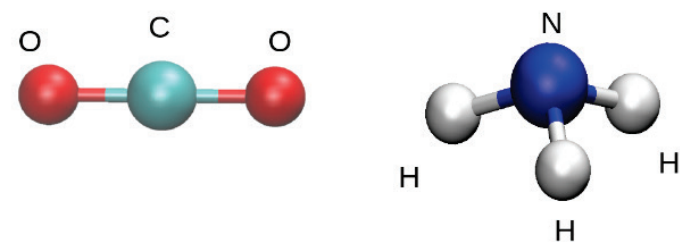

$\mathrm{CO}_{2}$

Figure 4-2. Molecular Structure and Atom Labels of a Carbon Dioxide Molecule (left) and an Ammonia Molecule (right)

Table 4-2. Non-bonded Parameters for Carbon Dioxide

\begin{tabular}{llrll}
\hline \hline Atoms & $\mathrm{m}[$ a.u. $]$ & $\mathrm{q}[e]$ & $\sigma[\AA]$ & $\varepsilon[\mathrm{kcal} / \mathrm{mol}]$ \\
\hline $\mathrm{C}$ & 12.010 & 0.6512 & 2.800 & 0.0559 \\
$\mathrm{O}$ & 15.999 & -0.3256 & 3.028 & 0.1597 \\
\hline \hline
\end{tabular}

The molecular structure of an ammonia molecule is shown on the right of Figure 4-2. The nitrogen atom is blue while the hydrogen atoms are white. Two sets of force field parameters are usually used for ammonia: OPLS all-atom (OPLS-AA) and TraPPE. OPLS-AA force field for ammonia was proposed by Rizzo and Jorgensen [147]. The TraPPE model of ammonia based on the 5-site model originally proposed by Impey and Klein [156], contains a fifth massless site carrying a negative charge and the bond lengths are rigid but the angles in the ammonia molecule are flexible [157]. The force field parameters for ammonia used in this work are from OPLS-AA [147] as follows, bond parameters: $k_{r}=434.0 \mathrm{kcal} /\left(\mathrm{mol} \cdot \AA^{2}\right), r_{\mathrm{eq}}=1.01 \AA$, angle parameters: $k_{\theta}=$ $43.60 \mathrm{kcal} /\left(\mathrm{mol} \cdot \mathrm{rad}^{2}\right), \theta_{e q}=106.4^{\circ}$, and the non-bonded parameters are listed in Table 4-3.

Table 4-3. Non-bonded Parameters for Ammonia

\begin{tabular}{ccccc}
\hline \hline Atoms & $\mathrm{m}$ [a.u.] & $\mathrm{q}[e]$ & $\sigma[\AA]$ & $\varepsilon[\mathrm{kcal} / \mathrm{mol}]$ \\
\hline $\mathrm{N}$ & 14.0065 & -1.02 & 0.17 & 3.42 \\
$\mathrm{H}$ & 1.008 & 0.34 & 0.0 & 0.0 \\
\hline \hline
\end{tabular}

\subsection{Simulation Systems and Details}

The relative solubilities of $\mathrm{CO}_{2}$ and $\mathrm{NH}_{3}$ were evaluated by considering the equilibrium volume expansion of simulation systems with added gas at different gas mole fraction under normal pressure of $1 \mathrm{~atm}$ which is the operational pressure of the proposed reaction heat exchanger. The prepared neat solvent systems consisted of 4096 water molecules and 2048 ethylene glycol molecules, respectively. To those neat solvent systems, a different number of gas molecules was added to achieve molar fraction $\mathrm{X}_{\text {gas }}$ of $1 \%, 5 \%, 10 \%, 20 \%, 30 \% \mathrm{CO}_{2}$ with additional $40 \%$ mole fraction of $\mathrm{NH}_{3}$ as shown in Table 4-4. In addition, the equilibrium volume expansion in $2 \%, 3 \%$, and $4 \%$ mole fraction of carbon dioxide in neat water was also considered. A number of water-

DISTRIBUTION STATEMENT A: Approved for public release. Distribution is unlimited. 
ethylene glycol systems were built corresponding to different ethylene glycol mole factions and, here, we report the results for two mixtures, ethylene glycol mole fraction of $10 \%$ and $75 \%$. The corresponding simulation systems were built using Packmol [89] by arranging 4096 water molecules and $480 \mathrm{EG}$ molecules in a random manner in the simulation box to achieve $\mathrm{X}_{\mathrm{EG}}=10 \%$ and 512 water molecules and $1536 \mathrm{EG}$ molecules for $\mathrm{X}_{\mathrm{EG}}=75 \%$.

All simulation systems were equilibrated for 100 ps under constant volume and temperature $(300 \mathrm{~K}$ or $333 \mathrm{~K})$, followed by 200 ps simulation time at constant pressure of $1 \mathrm{~atm}$ and constant temperature, and a final equilibration at constant volume and temperature for another $100 \mathrm{ps}$. The timestep of integration of the Newton's equations of motion was $0.001 \mathrm{ps}$. A snapshot of the simulated system was output every 500 simulation steps or every $0.5 \mathrm{ps}$. The equilibrium volume of the system was computed from the simulation box size at the end of the equilibration for each system. Periodic boundary conditions were used in all three dimensions to model bulk properties. The cutoff for the non-bonded interactions was $8 \AA$, smoothed over an additional angstrom [91]. Particle Mesh Ewald summation was used to account for the long-range Coulomb interactions beyond the cutoff. All simulation systems were built using Packmol [89] and all MD simulations were carried out using the Large-scale Atomic/Molecular Massively Parallel Simulator, LAMMPS [91].

Table 4-4. Number of Gas Molecules ( $\mathrm{CO}_{2}$ or $\left.\mathrm{NH}_{3}\right)$ in the Simulation Systems

(The simulations were set up to obtain the gas molar fraction $X_{\text {gas }}$ shown in the first row.)

\begin{tabular}{crrrrrr}
\hline \hline$X_{\text {gas }}$ & $1 \%$ & $5 \%$ & $10 \%$ & $20 \%$ & $30 \%$ & $40 \%$ \\
\hline In WAT & 41 & 202 & 440 & 1012 & 1716 & 2700 \\
In EG & 20 & 102 & 225 & 518 & 879 & 1350 \\
\hline \hline
\end{tabular}

\subsection{Results and Discussion}

\subsubsection{Equilibrium Volume and Equilibrium Volume Expansion}

The equilibrium volume of the simulation systems after the addition of $\mathrm{CO}_{2}$ or $\mathrm{NH}_{3}$ was compared to the original equilibrium volume of the neat solvent as a measure of the solubility of carbon dioxide and ammonia in pure water and pure ethylene glycol. The volume expansion of the liquid phase if defined as follows [158]:

$$
\frac{\Delta V}{V_{0}}=\frac{V-V_{0}}{V_{0}}
$$

where $V_{0}$ is the equilibrium molar volume of the neat solvent system and $V$ is the equilibrium molar volume of the liquid with added $\mathrm{CO}_{2}$ or $\mathrm{NH}_{3}$. The results for the equilibrium volume expansion at $300 \mathrm{~K}$ are shown in Figure 4-3. Here and in what follows, all volumes are permolecule. Figure 4-3 shows that both $\mathrm{CO}_{2}$ and $\mathrm{NH}_{3}$ are more soluble in ethylene glycol (EG) than in water, especially at higher mole fractions. Furthermore, $\mathrm{NH}_{3}$ is more soluble than $\mathrm{CO}_{2}$ in either water or ethylene glycol and the solubility of $\mathrm{CO}_{2}$ in water decreases at higher $\mathrm{CO}_{2}$ concentrations. Our simulations of the $\mathrm{CO}_{2}$ mole fractions of $20 \%$ and $30 \%$ showed spontaneous phase separation in the water - carbon dioxide system into water and $\mathrm{CO}_{2}$ phase. 


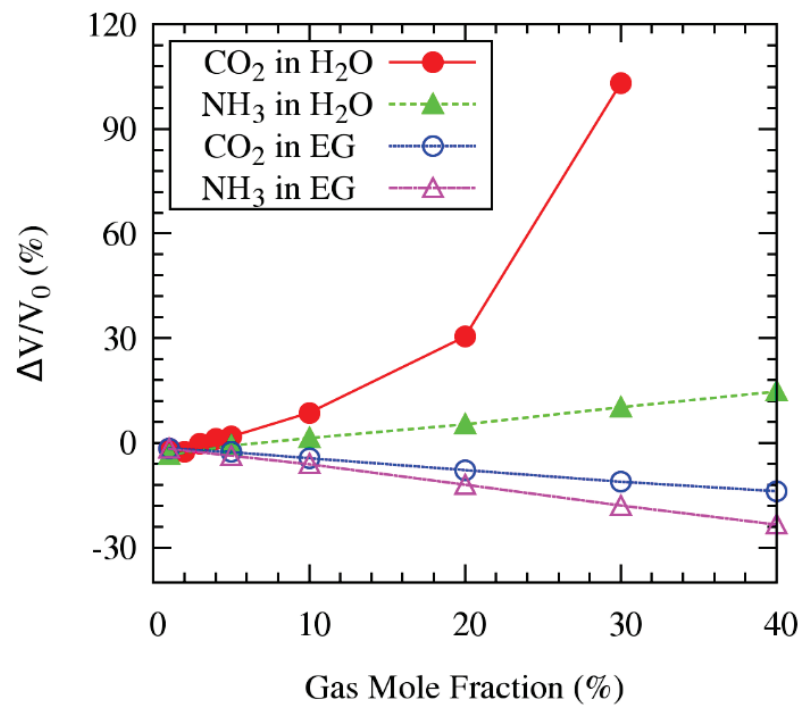

Figure 4-3: $\mathrm{CO}_{2}$ and $\mathrm{NH}_{3}$ Equilibrium Molar Volume Expansion as a Function of the Gas Mole Fraction in Water (Circles) and Ethylene Glycol (Triangles) at $300 \mathrm{~K}$.

(The values of the molar volume expansion are shown with filled symbols for $\mathrm{CO}_{2}$ and open symbols for $\mathrm{NH}_{3}$. The lines are provided as a guide to the eye.)

The solubility of carbon dioxide in water-ethylene glycol mixtures was also explored and Figure 4-4 compares the molar volume expansion when adding carbon dioxide to two water-EG mixtures, $10 \%$ mole fraction $\mathrm{EG}$ and $75 \%$ mole fraction $\mathrm{EG}$, with pure water and pure ethylene glycol. The results confirmed that the solubility of $\mathrm{CO}_{2}$ increases with the increase of the ethylene glycol mole fraction as it is clear from Figure 4-4.

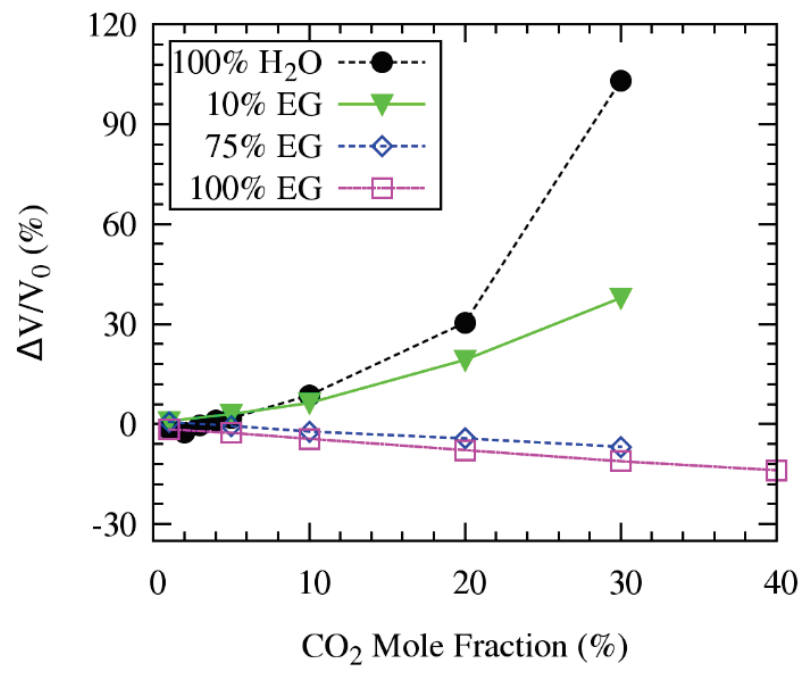

Figure 4-4: Concentration Dependence of the Equilibrium Molar Volume Expansion of $\mathrm{CO}_{2}$ in $\mathrm{H}_{2} \mathrm{O}$-EG Mixtures at $300 \mathrm{~K}$

(The equilibrium molar volume expansion as a function of $\mathrm{CO}_{2}$ mole fraction in water - ethylene glycol mixtures of increasing EG mole fraction at $300 \mathrm{~K}$. The lines are provided as a guide to the eye.) 
Figure 4-5 shows the temperature dependence of the equilibrium molar volume expansion of carbon dioxide and ammonia dissolved in pure water and pure ethylene glycol. A decrease in the solubility of ammonia was observed at the ammonium carbamate reaction temperature ( $333 \mathrm{~K}$ ) compared to the ammonia solubility at $300 \mathrm{~K}$ both in water and ethylene glycol. This effect has been observed experimentally $[159,160]$. In the case of carbon dioxide, however, the solubility in water decreases drastically at higher $\mathrm{CO}_{2}$ concentrations (above $10 \%$ mole fraction where the phase separation was observed) at the temperature of the decomposition reaction. Thus, the results of our simulations show that for the purposes of thermal management where the goal is to drive the reaction toward the thermal decomposition of ammonium carbamate and to efficiently remove the product gases, ethylene glycol is a poor choice of carrier fluid. Using a mixture of water and ethylene glycol would decrease the solubility of the product gases and may help with their removal. Screening of other solvents in which both $\mathrm{CO}_{2}$ and $\mathrm{NH}_{3}$ are less soluble as candidate carrier fluids for the thermal decomposition reaction of ammonium carbamate could be the subject of future work.
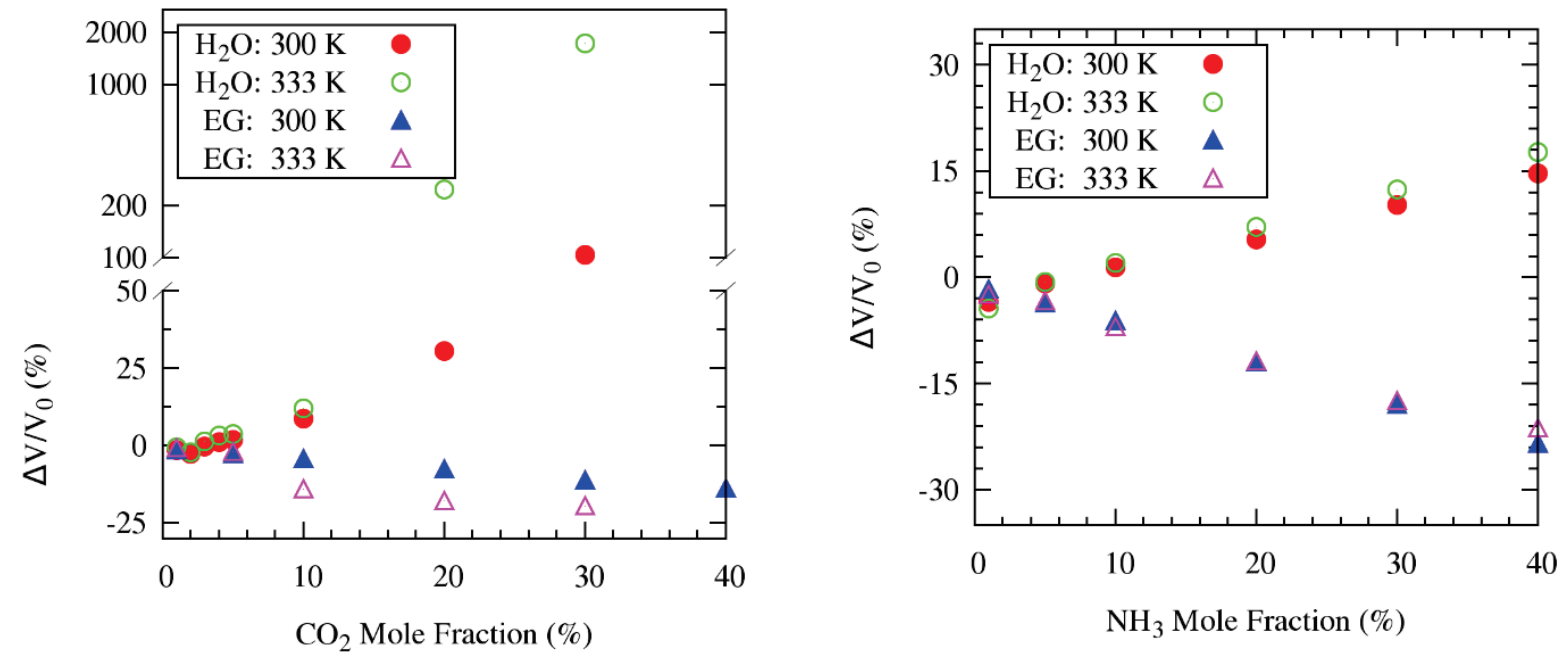

Figure 4-5: Equilibrium Molar Volume Expansion of $\mathrm{CO}_{2}$ and $\mathrm{NH}_{3}$ in $\mathrm{H}_{2} \mathrm{O}$ and EG

(Equilibrium molar volume expansion of $\mathrm{CO}_{2}$ (left) and $\mathrm{NH}_{3}$ (right) at different mole fraction in water (circles) and ethylene glycol (triangles) at $300 \mathrm{~K}$ (filled symbols) and $333 \mathrm{~K}$ (open symbols).)

\subsubsection{Temperature and Concentration Dependence of $\mathrm{CO}_{2}$ and $\mathrm{NH}_{3}$ Diffusivity in Water- Ethylene Glycol Mixtures}

After equilibration at pressure of 1 atm and room temperature $(300 \mathrm{~K})$ or the reaction temperature $(333 \mathrm{~K})$, classical $\mathrm{MD}$ simulations were performed for 1 nanosecond in NVE ensemble for all systems and the simulation results were used to compute the diffusivities of carbon dioxide and ammonia in the solutions from the mean squared displacement (MSD) of the center of mass of all gas molecules of each type as follows:

$$
D=\frac{1}{6} \lim _{t \rightarrow \infty} \frac{d}{d t}\left\langle|\vec{r}(t)-\vec{r}(0)|^{2}\right\rangle
$$

where $\left\langle|\vec{r}(t)-\vec{r}(0)|^{2}\right\rangle$ is the mean squared displacement averaged over all gas molecules of each type and over all time origins. The computed diffusion coefficients of carbon dioxide and ammonia

DISTRIBUTION STATEMENT A: Approved for public release. Distribution is unlimited. 
in pure ethylene glycol and pure water and in two mixtures, i.e., $10 \%$ and $75 \%$ molar fraction of ethylene glycol, at room temperature, are listed in Table $4-5$. The values for $20 \%$ and $30 \% \mathrm{CO}_{2}$ are in parentheses since phase separation was observed during the simulation and the computed diffusivities do not correspond to diffusion in water but rather in the gas phase. The computed values for the diffusivities of $\mathrm{CO}_{2}$ in neat water are comparable with the ones obtained by Moultos et al. [150] using the SPC/E water model even though the force field models used for carbon dioxide were different.

\section{Table 4-5: Values of the Computed Diffusion Coefficients of $\mathrm{CO}_{2}$ and $\mathrm{NH}_{3}$ in Water-EG Mixtures}

(The values are of the computed diffusion coefficients $\left(\times 10^{-5} \mathrm{~cm}^{2} / \mathrm{s}\right)$ of $\mathrm{CO}_{2}$ and $\mathrm{NH}_{3}$ in water-ethylene glycol mixtures at different gas mole fractions $\left(X_{\text {gas }}\right.$ ) at $300 \mathrm{~K}$. the first row lists the mole fraction $X_{E G}$

(\%) of ethylene glycol (EG) in the solvent mixture. The first column lists the values of the gas mole fraction in the solution.)

\begin{tabular}{lrrllll}
\hline \hline \multicolumn{1}{c}{$X_{E G} \rightarrow$} & \multicolumn{2}{c}{$0 \%$} & $10 \%$ & $75 \%$ & $100 \%$ & \\
\multicolumn{1}{c}{$X_{\text {gas }}$} & $\mathrm{CO}_{2}$ & $\mathrm{NH}_{3}$ & $\mathrm{CO}_{2}$ & $\mathrm{CO}_{2}$ & $\mathrm{CO}_{2}$ & $\mathrm{NH}_{3}$ \\
\hline $1 \%$ & 2.92 & 3.41 & 1.64 & 0.09 & 0.05 & - \\
$5 \%$ & 2.52 & 2.66 & 1.77 & 0.15 & 0.10 & 0.07 \\
$10 \%$ & 2.91 & 2.54 & 1.89 & 0.23 & 0.14 & 0.13 \\
$20 \%$ & $(7.28)$ & 2.81 & 3.52 & 0.43 & 0.28 & 0.25 \\
$30 \%$ & $(26.52)$ & 3.29 & 8.68 & 0.67 & 0.49 & 0.42 \\
$40 \%$ & & 3.79 & & & & 0.83 \\
\hline \hline
\end{tabular}

Additionally, Figure 4-6 compares the diffusivities on $\mathrm{CO}_{2}$ and $\mathrm{NH}_{3}$ in neat water and neat ethylene glycol at $300 \mathrm{~K}$ (left panel) and $333 \mathrm{~K}$ (right panel). Higher diffusivities are observed for both gases in water than in ethylene glycol. An increase of the diffusivity at elevated temperature was observed. For the case of carbon dioxide molar fraction in water above $20 \%$, when spontaneous phase separation was observed in our simulations, the diffusivity of $\mathrm{CO}_{2}$ increases dramatically because of the phase separation. Thus the computed values for the diffusion coefficients do not correspond to diffusivity in water but rather in the gas phase and, therefore, cannot be compared with the values computed for diffusion in the condensed phase. At low concentrations, the diffusivity of $\mathrm{CO}_{2}$ is somewhat higher than the diffusivity of $\mathrm{NH}_{3}$ in ethylene glycol, while in water both gases show similar diffusivities. Furthermore, the concentration dependence of the diffusivity of ammonia in both water and ethylene glycol is very weak at least to the extent of the concentrations that were considered in this study. Thus, even though the simulations did not account for the recombination reaction of carbon dioxide and ammonia, nor the reaction of the gases with the solvent, the computed diffusivity trends support the observations and conclusions made in the previous section when the equilibrium volume expansion was considered, namely, that in comparison to ethylene glycol, water is a better carrier fluid for the purposes of promptly removing the product gases of the thermal decomposition reaction of ammonium carbamate. 

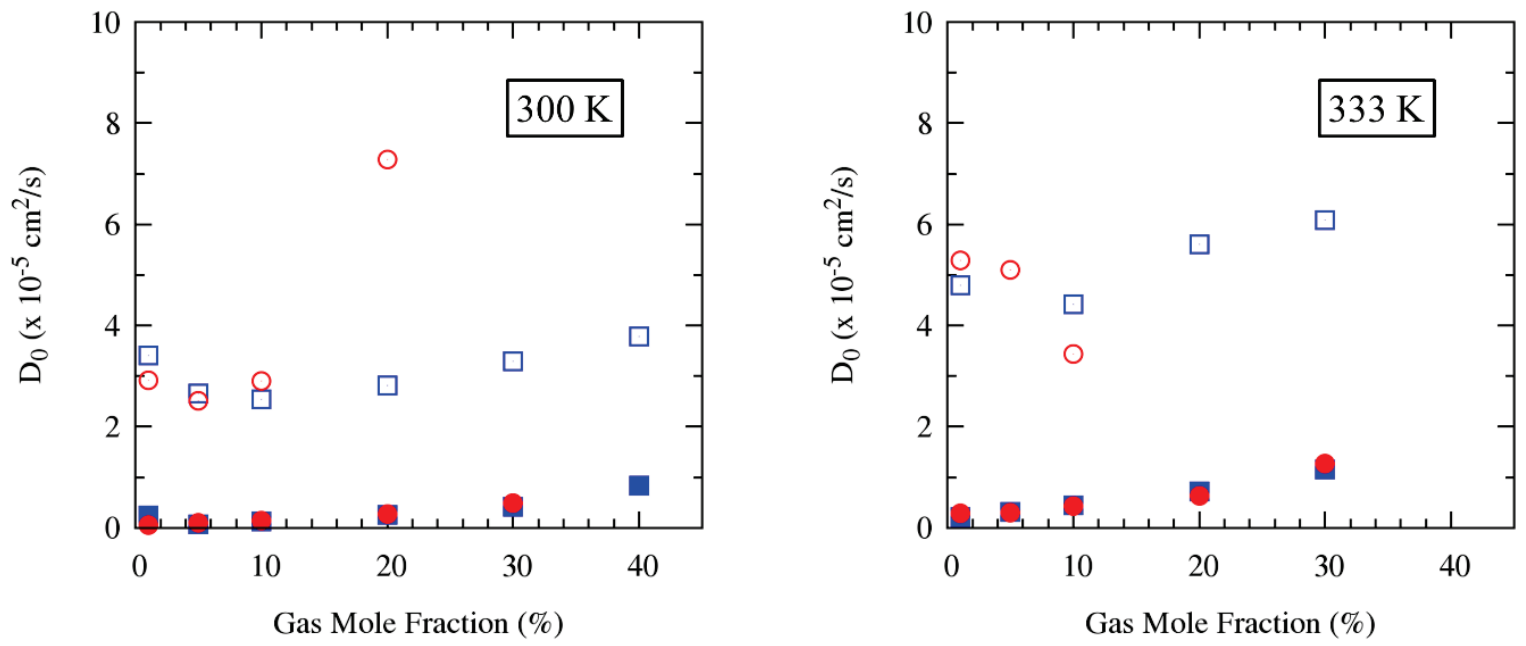

Figure 4-6: Diffusion Coefficients $\left(D_{0}\right)$

(Diffusion coefficients of $\mathrm{CO}_{2}$ (circles) and $\mathrm{NH}_{3}$ (squares) in neat water (open symbols) and ethylene glycol (filled symbols) at $300 \mathrm{~K}$ (left) and $333 \mathrm{~K}$ (right).)

\subsection{Conclusions}

An extensive set of MD simulations was carried out with the purpose of studying the liquid volume expansion and diffusion coefficients of the products of the thermal decomposition of ammonium carbamate, carbon dioxide and ammonia, in water and ethylene glycol at standard conditions and at the elevated temperature of the decomposition reaction. Solvent mixtures of water and ethylene glycol were also considered. The goal of this study was to compare the solvent compositions for the purposes of using the mixtures as carrier fluid in a heat exchanger utilizing the endothermic decomposition reaction of ammonium carbamate. The modeling and simulation results presented in this paper show that addition of hydroxyl groups, i.e., ethylene glycol, to aqueous solutions increases the solubility of carbon dioxide. This study points to the observation that compared to water, ethylene glycol is not as well suited to be a carrier fluid for the thermal decomposition reaction of ammonium carbamate. Addition of water could improve the properties of ethylene glycol as a carrier fluid by decreasing the solubility of the product gases and assisting with their removal. Alternatively, a different solvent or mixture can be a suitable carrier fluid in which either both ammonia and carbon dioxide are less soluble or their solubility decreases rapidly at higher gas mole fractions within the temperature range of the decomposition reaction. Thus, this study underlines that molecular dynamics simulations offer a powerful low-cost tool in fast throughput and engineering design that can be useful in a broader context of solvent screening for better solubility and heat transfer control beyond the more specific thermal decomposition reaction of ammonium carbamate. 


\section{MOLECULAR DYNAMICS STUDY OF OCTANE CONDENSATION COEFFICIENT AND EVAPORATIVE MASS FLUX AT LIQUID-VAPOR INTERFACES}

The mass accommodation coefficient $\alpha$ of liquids, including alkanes, is assumed to be 1.0 in fluid models of thin film evaporation even though it is known that it approaches unity only at low temperatures and decreases with increasing liquid temperature. The temperature range where $\alpha=$ 1 is valid, is yet to be understood in its entirety along with the nature of its dependence on liquid and substrate chemistry and surface roughness. In this work, the macroscopic mass accommodation coefficient of liquid octane was estimated by employing large scale equilibrium classical molecular dynamics simulations and computing the condensation probability as the ratio of the number of octane molecules that condense in the liquid phase to the number of octane molecules incident at the interface from the gas phase. At equilibrium, the evaporation coefficient is equal to the condensation (accommodation) coefficient. The effect of the model details on the estimated value of the accommodation coefficient was explored by using two different parameterizations of the octane molecule, the all-atom OPLS force field and the united-atom TraPPE. The condensation probabilities were estimated in the temperature range from $290 \mathrm{~K}$ to $350 \mathrm{~K}$. Compared to the all-atom molecular model, the united-atom model was found to consistently predict higher liquid density within the studied temperature range. The united-atom model also predicted higher condensation coefficients and evaporation rates at bulk liquid temperatures around room temperature (290 through $310 \mathrm{~K}$ ). Upper bounds of the evaporation rates per unit surface area were also estimated at the above temperatures within the two molecular models.

\subsection{Introduction}

Heat and mass transfer in thin evaporating liquid films is of interest in a variety of technological and industrial applications as part of the broader shift in thermal management towards exploiting the large heat fluxes provided by phase change processes. Numerical modeling of evaporative heat and mass transfer in thin films presents a multiscale problem where the intermolecular interactions between the substrate and the adsorbed thin film and within the liquid film and their effect on the evaporation should be correctly accounted for in the macro-scale model of fluid flow. The macroscopic mass accommodation coefficient, $\alpha_{c}$, can be defined as the condensation probability at the liquid-gas interface, equal to the ratio of the number of molecules that condense in the liquid and the total number of gas-phase molecules that reach the gas-liquid interface [161, 162]. The accommodation coefficient appears as an empirical parameter in the fluidic models of thin film evaporation and its value is customarily taken to be one but the question of the range of validity of this assumption has not been adequately explored. The atomic-scale knowledge of the molecular structure and intermolecular interactions of the evaporating liquid allow for inferring the value of the accommodation coefficient via molecular dynamics simulations. At equilibrium, the evaporation and condensation are reversible processes and can be studied by molecular dynamics simulations and statistical methods. Since at equilibrium, the evaporation and condensation coefficients are the same, throughout this work, mass accommodation and condensation coefficient are used interchangeably. The mass uptake and interactions at the liquid-gas interfaces are important and studied in other areas including physics of atmospheric aerosols [162-164].

MD simulations [14] have proven to be a powerful tool for providing atomic-scale insight into the structure, dynamics, and energetics underlying interfacial properties and processes. Molecular dynamics simulations have been applied to study the condensation coefficient of

DISTRIBUTION STATEMENT A: Approved for public release. Distribution is unlimited. 
Lennard-Jones liquids [165-167], water [162, 163, 168-175] and, more recently, to melting and condensation of octane [161,165, 176-178]. Still, molecular modeling is underutilized in thermal management. There have been two major approaches to obtaining the water evaporation/condensation coefficient from atomistic-detail simulations. One approach is through quantum mechanical methods - to consider water evaporation a rare event and to study it by means of transition path sampling (TPS) [171, 172, 175]. Another approach is to study condensation probabilities statistically $[162,170,173,174]$ and this is the approach adopted in this work, specifically, so that the results are comparable with the recent results for octane at higher temperatures [161]. The two approaches predict accommodation coefficient of water very close to unity independent of the specific water model being used. While the accommodation coefficient of the water has been extensively studied, there are not many studies of the condensation or evaporation of non-polar liquids and hydrocarbons [161, 165, 179], and the sampled temperature range does not include room temperature which is important for cooling of electronics.

In this study, we explore the validity of the common assumptions for the value of accommodation coefficient at vapor-liquid interfaces by studying the condensation probability of liquid octane around room temperature via classical molecular dynamics simulations. We explore the effect the molecular parameterization models have on the interfacial properties and on the computed macroscopic accommodation coefficient by using two force fields. A united-atom model with no partial charges and an all-atom model with partial atomic charges were used for octane and simulations of liquid octane were performed in a slab geometry at liquid temperature of 290 $\mathrm{K}, 300 \mathrm{~K}, 310 \mathrm{~K}, 330 \mathrm{~K}$, and $350 \mathrm{~K}$.

\subsection{Molecular Model of Octane}
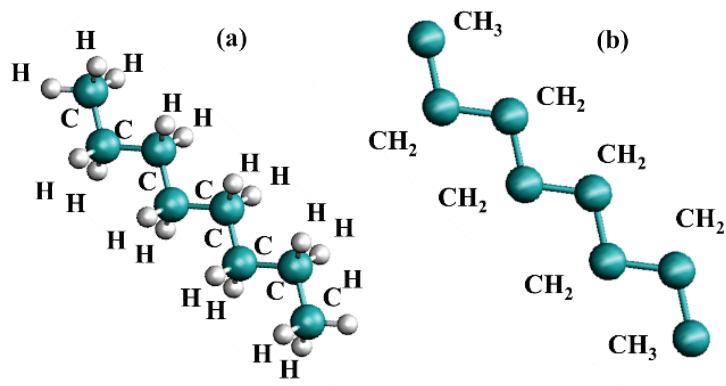

Figure 5-1: All-Atom and United-Atom Models of an Octane Molecule

((a) All-atom model of an octane molecule. The carbon atoms are shown in cyan and the hydrogen atoms are shown in gray. (Not all hydrogen atoms are labeled.); (b) United-atom model of an octane molecule.

Both the terminal methyl groups $\left(\mathrm{CH}_{3}\right)$ and the methylene groups $\left(\mathrm{CH}_{2}\right)$ are shown as united-atoms.)

The atomic structure of a single octane molecule $\mathrm{C}_{8} \mathrm{H}_{18}$ is illustrated in Figure 5-1 (a). An all-atom model of octane assigns to each atom a set of parameters (FF parameters), which describe how the atom interacts with the other atoms within the molecule (bonded interactions: bond, angle, and dihedral parameters) as well as with atoms belonging to other molecules (non-bonded interactions: Coulomb and Lennard-Jones). The bonded interactions contribution to the potential energy of the system comes from harmonic bond stretching, $K_{r}\left(r-r^{e q}\right)^{2}$, harmonic bond bending $K_{\theta}\left(\theta-\theta^{e q}\right)^{2}$, and the torsional energy for a dihedral angle $\varphi$ defined by four bonded atoms, $V^{(0)}+V^{(1)}(1+\cos \varphi)+V^{(2)}(1-\cos 2 \varphi)+V^{(3)}(1+\cos 3 \varphi)$. Here $K_{r}$ and $K_{\theta}$ are the spring constants in the harmonic description pf bond stretching and angle bending, respectively; $r^{e q}$ and

DISTRIBUTION STATEMENT A: Approved for public release. Distribution is unlimited. 
$\theta^{e q}$ are the equilibrium bod length and equilibrium angle, respectively; and $V^{(0)}, V^{(1)}, V^{(2)}$, and $V^{(3)}$ are the torsion parameters. The non-bonded contribution to the potential energy is given in Eq. $(5-1)$ :

$$
V=\sum_{i<j}^{a t o m s}\left[\frac{q_{i} q_{j}}{4 \pi \varepsilon_{0} R_{i j}}+4 \epsilon_{i j}\left(\left(\frac{\sigma_{i j}}{R_{i j}}\right)^{12}-\left(\frac{\sigma_{i j}}{R_{i j}}\right)^{6}\right)\right]
$$

The first part in the above Eq. (5-1) is the Coulomb electrostatic interaction between atoms $i$ and $j$ with charges $q_{i}$ and $q_{j}$, respectively, the distance between the atoms is $R_{i j}$, and $\varepsilon_{0}=8.9 \times$ $10^{-12} \mathrm{~F} / \mathrm{m}$ is the permittivity of free space. The second part in Eq. (1) is the Lennard-Jones interaction between each pair of atoms, where $\epsilon_{i j}$ and $\sigma_{i j}$ are the minimum of the interaction energy and the zero-energy separation, respectively. Therefore, the force field parameters of a model, together with the partial charges assigned to each atom, define the interaction energy of a system of molecules. In this work, we used both the all-atom OPLS [75, 180] and the united-atom TraPPE [181, 182] octane force fields to model liquid-vapor interface and condensation and evaporation of octane. The values of the force field parameters are listed in Table 5-1. In a molecular dynamics simulation, Newton's equations of motion are solved for each atom in the interaction potential with the rest of the atoms in the system, $V_{\text {bonded }}+V_{\text {non-bonded }}$. The trajectories of all atoms are output with predefined frequency and can be further analyzed to extract relevant information. A united-atom model of octane, for example, treats each of the methylene $\left(\mathrm{CH}_{2}\right)$ and methyl $\left(\mathrm{CH}_{3}\right)$ groups as larger atoms of new types, Figure 5-1 (b). Grouping the hydrogen atoms together with the carbon atom to which they are bonded allows for an increase of the integration timestep when solving the equations of motion while simultaneously reducing the total number of interaction sites in the simulated system. At the same time, it is important to assess how well a united-atom model reproduces experimental properties of the molecular system in comparison with an all-atom model. The octane molecule is a non-polar molecule and the TraPPE force field describes it via non-charged united atoms while the all-atom OPLS model assigns partial charges on each atom, which add up to a neutral molecule. 
Table 5-1: Force Field Parameters

(The united-atom TraPPE [181, 182] and the all-atom OPLS [75, 180] are used in this section. The units

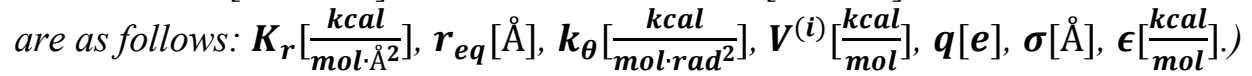

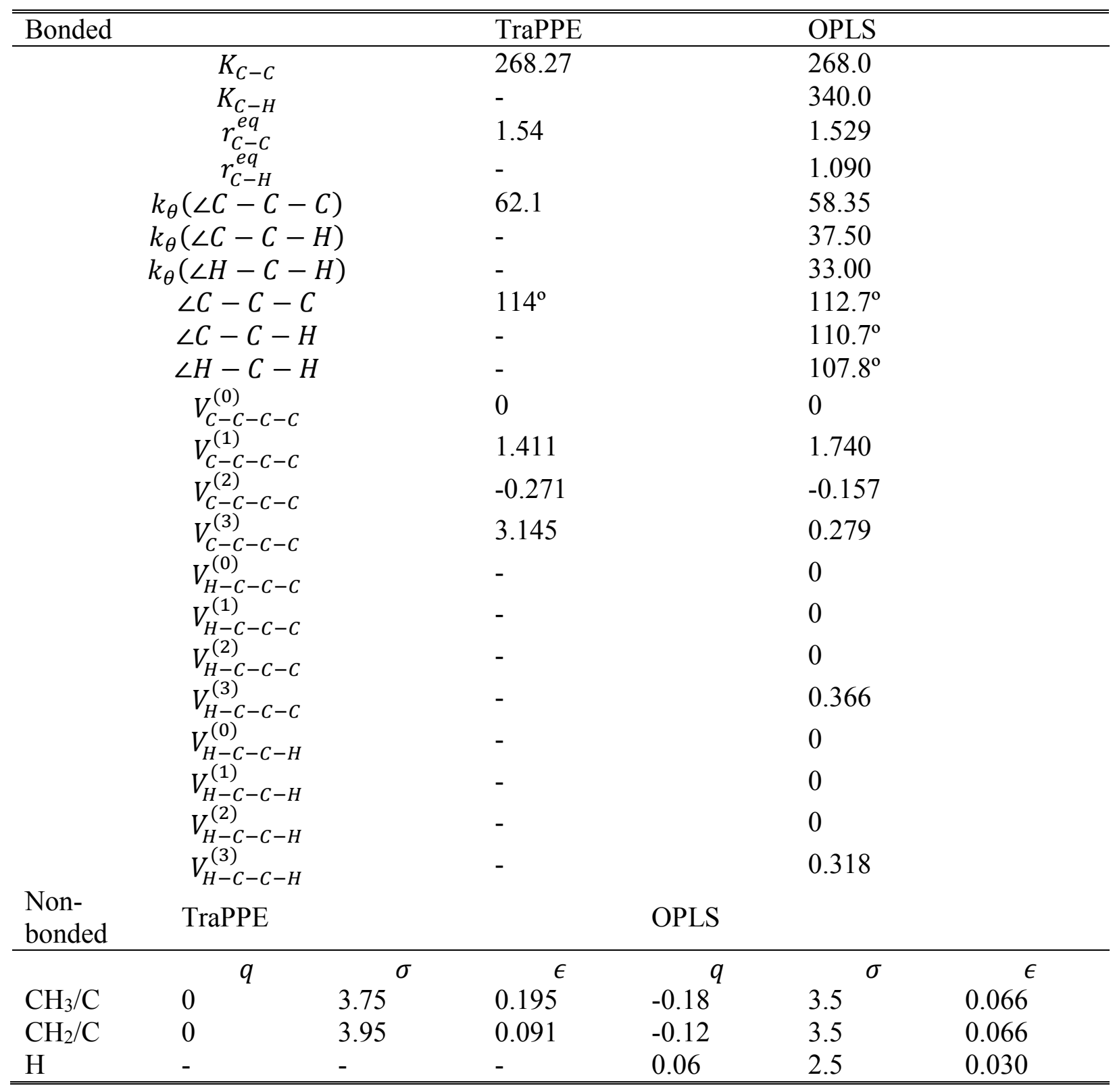

\subsection{Molecular Dynamics Simulation Setup}

In this section, the simulation of liquid-vapor interfaces was carried out in planar slab geometry [161, 168, 183]. Using the program Packmol [89], $2.224 \times 10^{3}$ united-atom (UA) octane molecules were arranged in a slab geometry in a rectangular simulation box of approximate size $100 \AA(x) \times 100 \AA(y) \times 300 \AA(z)$. An analogous simulation system containing 506 all-atom (AA) octane molecules was prepared in a simulation box of approximate size: $40 \AA(x) \times 40 \AA(y) \times 300$ $\AA(z)$. The liquid-vacuum interfaces were within the $x y$-plane and perpendicular to the $z$-direction. The width of the liquid slabs was about $80 \AA$ for the AA systems and $60 \AA$ for the UA systems,

DISTRIBUTION STATEMENT A: Approved for public release. Distribution is unlimited. 
which in both cases provides sufficient separation between the liquid-vapor interfaces. Additionally, the periodic boundary conditions in all three directions allowed for the simulation of slabs of liquid separated by about $200 \AA$ of vacuum. All simulations in this work were performed using the Large-scale Atomic/Molecular Massively Parallel Simulator, LAMMPS [91]. After energy minimization, the systems were equilibrated at constant temperature and volume, and timestep of $10^{-3}$ ps for at least $1 \mathrm{~ns}$ and up to $10 \mathrm{~ns}$. Snapshots from the equilibration simulation, stored 100 ps apart, were used as statistically independent staring configurations, a standard approach used before, see for example Viecely et al. [162]. The scattering simulations which provided the statistics of incident, condensed, and scattered molecules were run without a thermostat and in energy conserving ensemble. The scattering simulations were carried out by positioning a single molecule from the bulk at specified spatial coordinates in the gas phase halfway between the liquid slabs while the velocities of the molecule and its atoms were preserved (option available in LAMMPS) which does not change the total kinetic energy of the simulated system and only the potential energy is slightly affected but the differences are within the fluctuations of the total energy or system temperature. Figure 5-2 illustrates this by sampling the system temperature during arbitrary collections of 100 energy-conserving scattering simulations of 1-ns length each and each followed by constant temperature equilibration run of $0.2 \mathrm{~ns}$ at the respective target temperature. Figure 5-2 shows that the temperature fluctuations within each the energy conserving runs and between them, are within the usual limits for molecular dynamics simulations. This allowed us to pull individual molecules from the liquid slab and position them one at a time in the vapor phase equidistant from the two liquid-vapor interfaces. Since the molecules were pulled from the liquid phase, their velocities sampled the velocity distribution of the molecules in the bulk liquid which was previously equilibrated at constant volume and the target temperature. Furthermore, the $x$ - and $y$-position of the molecule was not changed, only the $z$-position and this allowed for the incident molecules to essentially randomly sample the $(x, y)$ starting positions within the simulation box. After positioning the molecule in the middle of the vapor phase, MD simulation was run for $1 \mathrm{~ns}$ in NVE ensemble to ensure energy conserving dynamics and collisions. Between the runs, the systems were again equilibrated in constant volume simulation and constant target temperature. The condensation probability, $\alpha_{c}$, was computed as $\alpha_{c}=\frac{N_{\text {condensed }}}{N_{\text {incident }}}$, where $N_{\text {condensed }}$ was the total number of adsorbed and absorbed octane molecules, and $N_{\text {incident }}$ included all adsorbed, absorbed, scattered, and desorbed octane molecules [164, 184]. Five bulk temperatures, $290 \mathrm{~K}, 300 \mathrm{~K}, 310 \mathrm{~K}, 330 \mathrm{~K}$, and $350 \mathrm{~K}$, were considered to explore the range of existing experimental setup [185] and to extend the range of available literature data [161]. All simulations were repeated at the five temperature values to assess the effect of temperature on the condensation and evaporation of octane. The condensation probability or macroscopic condensation coefficient is expected to decrease with increasing temperature and approach one at low temperatures $[161,186]$ and this work adds to the recent study of Nagayama et al. [161] by exploring effects within a temperature range not considered previously. 


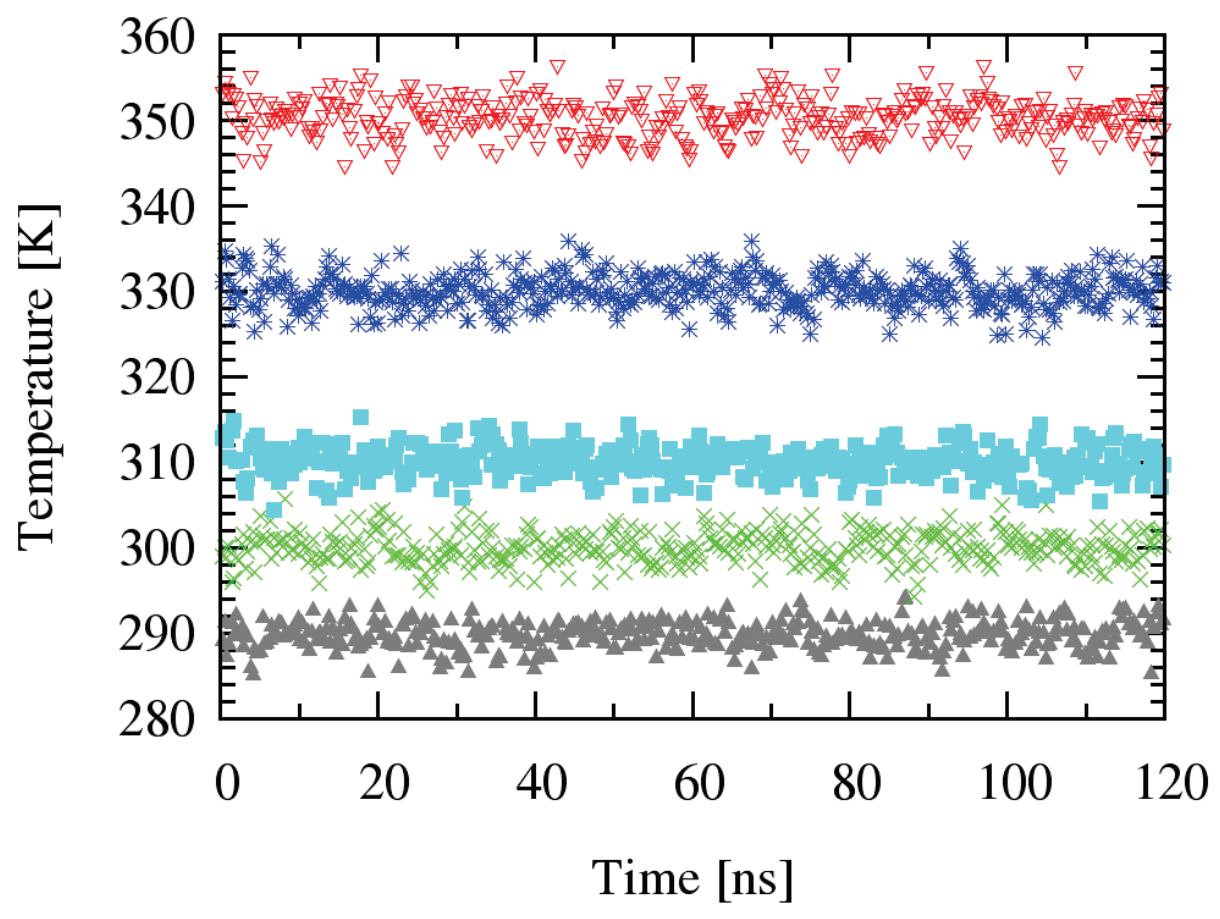

Figure 5-2: Evolution of the Systems Temperature

(Evolution of the temperature of the simulated systems during 100 scattering and 100 equilibrium simulations with TraPPE FF. Each 1-ns run is shown followed by a shorter equilibration run at the respective target temperature.)

\subsection{Simulation Results and Discussion}

\subsubsection{Structure of the Liquid-vapor Interfaces}

The density profiles in $z$-direction were computed over 1-ns simulation runs for each simulation temperature. The liquid-vapor interfaces can be further characterized by fitting the density profiles with the expression in the following equation $[164,187,188]$ :

$\rho(z)=\frac{\rho_{l}+\rho_{v}}{2}-\frac{\rho_{l}-\rho_{v}}{2} \tanh \left(\frac{z-z^{(G D S)}}{\delta}\right)$

which allows us to determine the position of the Gibbs dividing surface, $z^{(G D S)}$, as well as the liquid density within the slab, $\rho_{l}$, and the width of the interface, $\delta$, for each 1-ns simulation. The vapor density $\rho_{v}$ was taken to be zero. The instantaneous position of the Gibbs dividing surface (GDS) and the interface width can be used to determine if an incident molecule has reached the interface and if this molecule has adsorbed or reflected off the liquid surface, see Section 5.4.2 in what follows. Comparisons of the averaged liquid densities and interfacial widths predicted by the two octane models at the five simulation temperatures is listed in Table 5-2 and Table 5-3, respectively, and show that the non-polar united atom TraPPE model predicts somewhat higher liquid densities and wider interfacial widths compared to the all-atom OPLS force field.

DISTRIBUTION STATEMENT A: Approved for public release. Distribution is unlimited. 
Table 5-2: Liquid Densities, in $\mathrm{g} / \mathrm{cm}^{3}$, Computed at the Five Simulation Temperatures

\begin{tabular}{cccccc}
\hline \hline & $290 \mathrm{~K}$ & $300 \mathrm{~K}$ & $310 \mathrm{~K}$ & $330 \mathrm{~K}$ & $350 \mathrm{~K}$ \\
\hline TraPPE & 0.695 & 0.687 & 0.676 & 0.658 & 0.638 \\
OPLS & 0.693 & 0.685 & 0.672 & 0.653 & 0.630 \\
\hline \hline
\end{tabular}

Table 5-3: Interfacial Widths, in $\AA$, Computed at the Five Simulation Temperatures

\begin{tabular}{cccccc}
\hline & $290 \mathrm{~K}$ & $300 \mathrm{~K}$ & $310 \mathrm{~K}$ & $330 \mathrm{~K}$ & $350 \mathrm{~K}$ \\
\hline TraPPE & 4.0 & 4.3 & 4.5 & 5.2 & 5.7 \\
OPLS & 3.3 & 3.5 & 3.6 & 4.3 & 4.8 \\
\hline \hline
\end{tabular}

A plot of the density profiles of one representative liquid-vapor interface modeled with TraPPE at the five liquid temperatures is shown in Figure 5-3 and illustrates the slight decrease of liquid slab density and simultaneously increase of the interfacial width with increasing temperature. An analogous trend is observed for the OPLS force field.

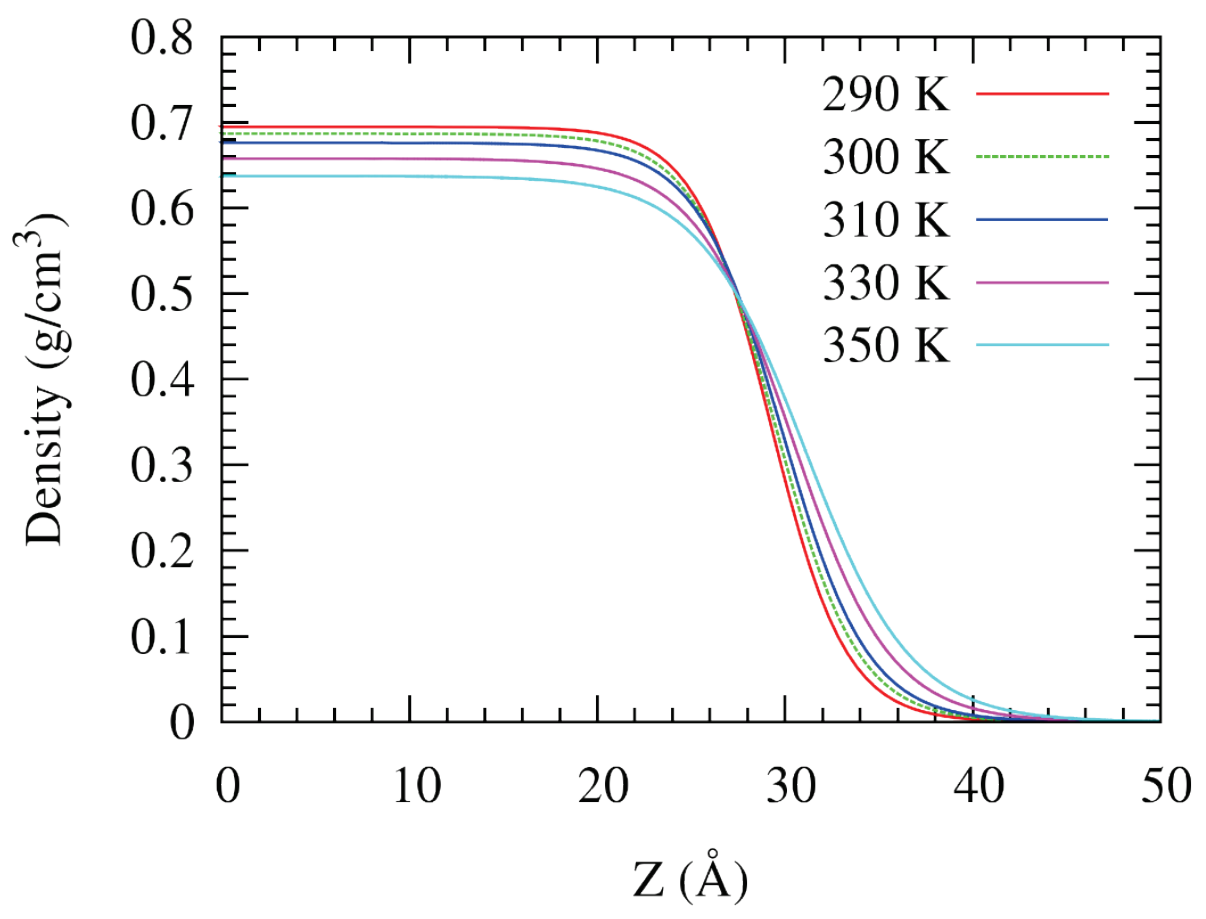

Figure 5-3: Density Profiles

(Density profiles of the system simulated with the united-atom TraPPE force field at $290 \mathrm{~K}, 300 \mathrm{~K}, 310 \mathrm{~K}$, $330 \mathrm{~K}$, and $350 \mathrm{~K}$.)

The bulk densities of octane, $0.694 \mathrm{~g} / \mathrm{cm}^{3}$ predicted by the TraPPE model and $0.685 \mathrm{~g} / \mathrm{cm}^{3}$ for the OPLS model, are in good agreement with the experimental liquid octane density of 0.703 $\mathrm{g} / \mathrm{cm}^{3}$ at normal conditions, temperature of $300 \mathrm{~K}$ and pressure of $1 \mathrm{~atm}$. When MD simulations are performed in a slab geometry, no external pressure is enforced and the observed liquid density is entirely due to the cohesive properties of the used force field. This is why, the observed liquid densities in slab geometry are slightly lower than the bulk densities at normal conditions.

DISTRIBUTION STATEMENT A: Approved for public release. Distribution is unlimited. 


\subsubsection{Condensation Probabilities, Evaporation Rates, and Scattering Events}

The evolution of the z-coordinate of each of the incident molecules was tracked during the 1-ns simulations. Figure 5-4 shows the time evolution of the z-position of all incident molecules which originated from the gas phase, halfway between the liquid surfaces for the system modeled with the TraPPE force field and equilibrated at $300 \mathrm{~K}$. These trajectories were obtained from independent simulations and were shown in different colors for clarity. From Figure 5-4, it can be observed that a continuum of velocities was well sampled. During the simulation, some of the incident molecules were lost due to insufficient velocity in z-direction which made the 1-ns simulation insufficient for the octane molecule to reach one of the liquid surfaces. The small speed in z-direction was due either to the initial conditions or to scattering from other gas-phase octane molecules. As a result the total number of incident molecules varies somewhat for the different simulation system. Gas phase molecules due to spontaneous evaporation events or desorption were observed for both octane models.

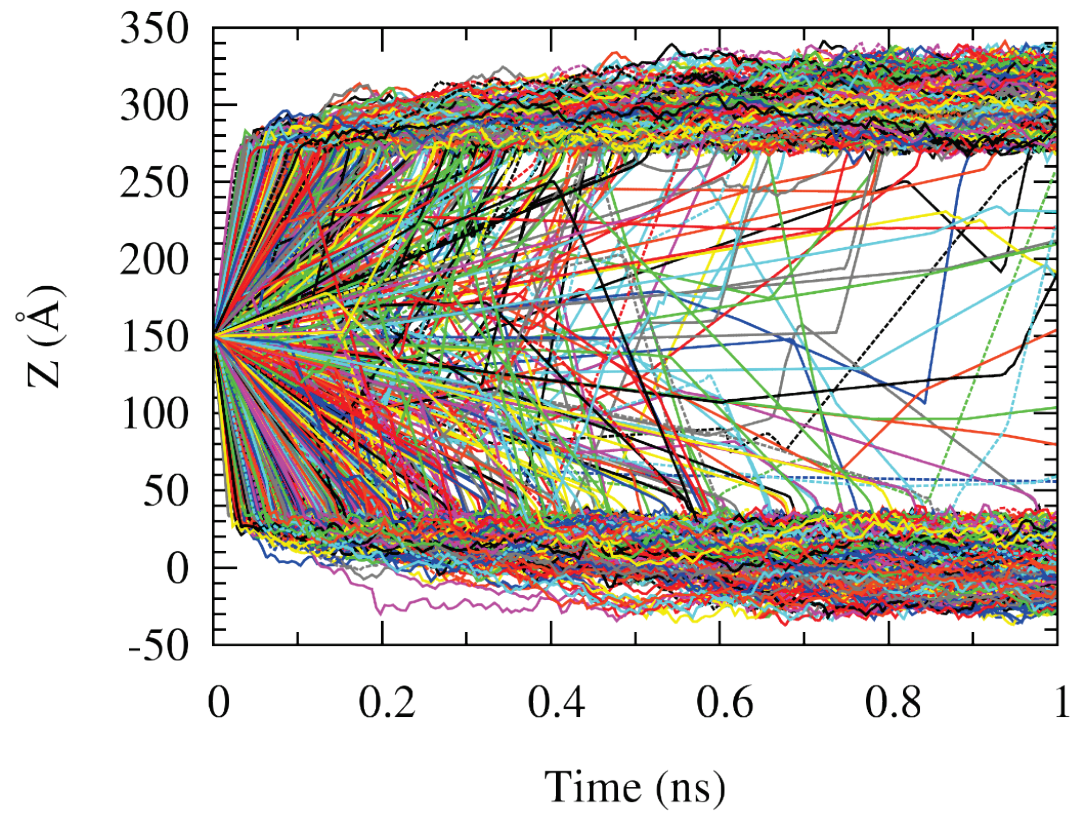

Figure 5-4: Time Evolution of the Z-coordinates of the Incident Molecules

(The time evolution of all incident gas phase octane molecules with starting positions halfway (150 A) between the liquid surfaces at approx. $30 \AA$ and $270 \AA$. The position of each molecule was determined through an independent run and the trajectories of different molecules were shown in different colors. The systems were originally equilibrated at $300 \mathrm{~K}$ and modeled with the united-atom TraPPE force field.)

The evolution of the z-position of all octane molecules was tracked and plotted for each 1ns simulation. Inspection of the collection of the evolution of the z-positions of the octane molecules for each temperature and octane model shows that the spontaneous evaporation increases with the increase of the liquid temperature and that the desorption or spontaneous evaporation events are more frequent for the non-polar UA force field. The evaporation, condensation, and scattering events were all considered at the instantaneous liquid-vapor interface defined by the Gibbs dividing surface, $z^{(G D S)}$, Eq. (5-2). More specifically, an octane molecule was considered: adsorbed at the liquid-vapor interface if $z^{(G D S)}-2 \delta \leq z \leq z^{(G D S)}+2 \delta$; condensed in the bulk if $z<z^{(G D S)}-2 \delta$; reflected or scattered off the interface if $z>z^{(G D S)}+$

DISTRIBUTION STATEMENT A: Approved for public release. Distribution is unlimited. 
$2 \delta$ after interacting with it, i.e., having been adsorbed for no more than $2 \mathrm{ps}$; and evaporated or desorbed if $z>z^{(G D S)}+2 \delta$ after being adsorbed or in the bulk for more than $2 \mathrm{ps}$. A summary of the observed evaporation and condensation events is shown in Table 5-4. Due to the slab geometry, the surface area available for evaporation, approx. $3.708 \times 10^{3} \AA^{2}$ for the AA model systems and $2.1383 \times 10^{4} \AA^{2}$ for the UA model systems, is twice the area of the simulation box in $x y$-plane. With the temperature increase, the number of evaporating events increases which increases of the number of incident molecules. As a result, the number of observable events improves at higher temperature. The statistical results were further analyzed using reservoir sampling with replacement, a bootstrapping technique, to randomly generate $10^{4}$ independent sets of $100 \mathrm{~ns}$ total simulation time for each temperature and force field. The accommodation coefficient and evaporative mass flux were computed for each 100 -ns simulation set. The observed statistical distributions of the accommodation coefficient and the evaporative mass flux were normalized and fitted with Gaussian distributions for each temperature and octane model. Normalized distributions of the accommodation coefficient are shown on Figure 5-5 for OPLS-AA model, together with the corresponding Gaussian fits (shown with lines).

Table 5-4: Summary of the Total Number of Observed Incident Molecules $N_{\text {inc, }}$ Evaporation ( $\mathbf{N}_{\text {evap}}$ ) and Condensation ( $\left.\mathbf{N}_{\text {cond }}\right)$ Events, and Total Observation Time $\tau$ [ns]

\begin{tabular}{llrrrr}
\hline \hline & & $\mathrm{N}_{\text {inc }}$ & $\mathrm{N}_{\text {cond }}$ & $\mathrm{N}_{\text {evap }}$ & $\tau$ \\
\hline $290 \mathrm{~K}$ & UA & 3010 & 2982 & 2759 & 151 \\
$300 \mathrm{~K}$ & $\mathrm{UA}$ & 5062 & 4996 & 4750 & 169 \\
$310 \mathrm{~K}$ & $\mathrm{UA}$ & 11302 & 11138 & 10940 & 262 \\
$330 \mathrm{~K}$ & $\mathrm{UA}$ & 1759 & 4574 & 4296 & 199 \\
$350 \mathrm{~K}$ & $\mathrm{UA}$ & & 12504 & 11692 & 295 \\
& & & & \\
$290 \mathrm{~K}$ & $\mathrm{AA}$ & 2140 & 2114 & 1508 & 610 \\
$300 \mathrm{~K}$ & $\mathrm{AA}$ & 2929 & 2882 & 2399 & 577 \\
$310 \mathrm{~K}$ & AA & 3987 & 3910 & 3420 & 496 \\
$330 \mathrm{~K}$ & AA & 3523 & 3390 & 3174 & 194 \\
$350 \mathrm{~K}$ & AA & 10111 & 9598 & 8880 & 272 \\
\hline \hline
\end{tabular}




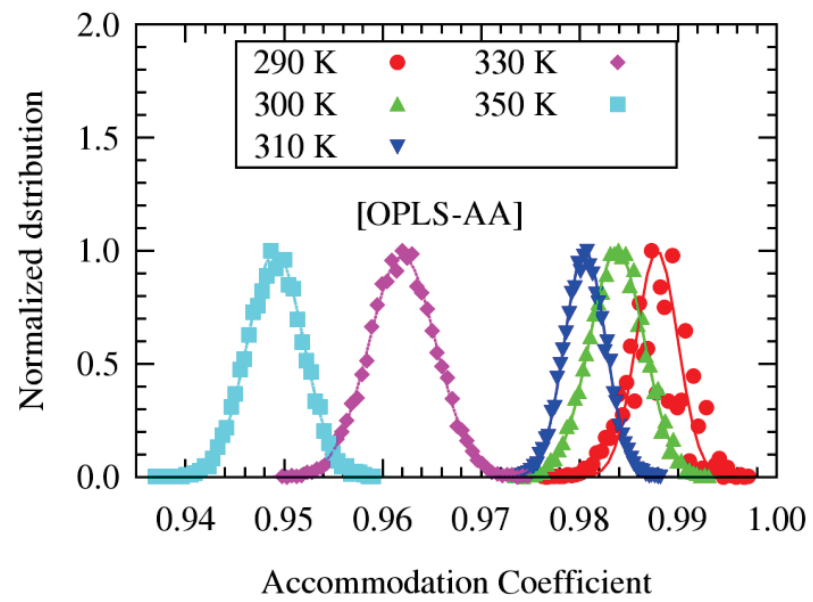

Figure 5-5: Normalized Distributions of the Computed Accommodation Coefficient

(Normalized distributions of the computed accommodation coefficient from $10^{4}$ randomized samples of duration $100 \mathrm{~ns}$ from simulations with the all-atom OPLS-AA force field at the five simulated temperatures. The corresponding Gaussian fits are also shown with lines.)

The condensation probabilities of octane were estimated at the five different values of the equilibrium liquid temperature: $290 \mathrm{~K}, 300 \mathrm{~K}, 310 \mathrm{~K}, 330 \mathrm{~K}$, and $350 \mathrm{~K}$. The results obtained with the two force field models are shown in Table 5-5. A graphical representation of our results together with the available literature values [161] are shown in Figure 5-6. The results of this work extend into the room temperature range and show the accommodation coefficient leveling off and approaching unity at low temperatures. The difference in the computed values between the two models, the non-polar united-atom model and the all-atom model with partial charges, is less than $1 \%$ and shows that for a prediction of the macroscale accommodation coefficient, the use of a united-atom model is well justified by the computational savings and achieved accuracy. Our results extend and complement the available values at higher temperatures [161] and align in a very good agreement within the expectation that at lower temperatures, the macroscopic condensation probability approaches unity.

Table 5-5: Macroscopic Accommodation Coefficient $\left(\alpha_{c}\right)$ and Evaporative Mass Flux of Liquid Octane

(The results were computed at different equilibrium temperatures and with the two molecular force field models.)

\begin{tabular}{llllll}
\hline \hline & $290 \mathrm{~K}$ & $300 \mathrm{~K}$ & $310 \mathrm{~K}$ & $330 \mathrm{~K}$ & $350 \mathrm{~K}$ \\
\hline UA & 0.999 & 0.998 & 0.997 & 0.995 & 0.983 \\
AA & 0.998 & 0.996 & 0.995 & 0.994 & 0.984 \\
\hline \hline
\end{tabular}

During the simulations, the number of evaporation events, i.e., octane molecules leaving the bulk phase, was also recorded and used to provide an estimate of the octane evaporative mass flux in $\left[\mathrm{g} /\left(\mathrm{cm}^{2} \mathrm{~s}\right)\right]$ and its temperature and model dependence, Table 5-6. The evaporative mass flux increased with increase of the liquid temperature and predictions of the two octane models were consistent below $350 \mathrm{~K}$. At the temperature of $350 \mathrm{~K}$, the non-polar united atom model predicted somewhat lower mass flux, $17.6 \mathrm{~g} /\left(\mathrm{cm}^{2} \mathrm{~s}\right)$ vs. $19.7 \mathrm{~g} /\left(\mathrm{cm}^{2} \mathrm{~s}\right)$ predicted by the all-atom model. Additional simulations and temperatures between $330 \mathrm{~K}$ and $350 \mathrm{~K}$, and above $350 \mathrm{~K}$ with

DISTRIBUTION STATEMENT A: Approved for public release. Distribution is unlimited. 
the both models could be used to evaluate if the observed difference in the predicted evaporative mass flux at $350 \mathrm{~K}$ reflects a fluctuation or an inherent difference between the two models. The evaporation events observed in our molecular dynamics simulations correspond to evaporation in vacuum (no atmospheric pressure present) but also in confinement (the distance between the liquid slabs was about $300 \AA$ ) and, therefore, represent an idealized situation that does not directly correspond to free surface evaporation but is more applicable to microfluidic systems where evaporation occurs in confinement. To the best of our knowledge, this is the first theoretical estimate of the evaporative mass flux of octane obtained from molecular dynamics simulations.

\section{Table 5-6: Evaporative Mass Flux (in $\mathrm{g} / \mathrm{cm}^{2} \mathrm{~s}$ ) of Liquid Octane}

(Computed at different equilibrium temperatures and with the two molecular FF models.)

\begin{tabular}{cccccc}
\hline \hline & $290 \mathrm{~K}$ & $300 \mathrm{~K}$ & $310 \mathrm{~K}$ & $330 \mathrm{~K}$ & $350 \mathrm{~K}$ \\
\hline UA & 1.7 & 2.4 & 4.2 & 9.0 & 17.6 \\
AA & 1.3 & 2.3 & 3.8 & 9.0 & 19.7 \\
\hline \hline
\end{tabular}

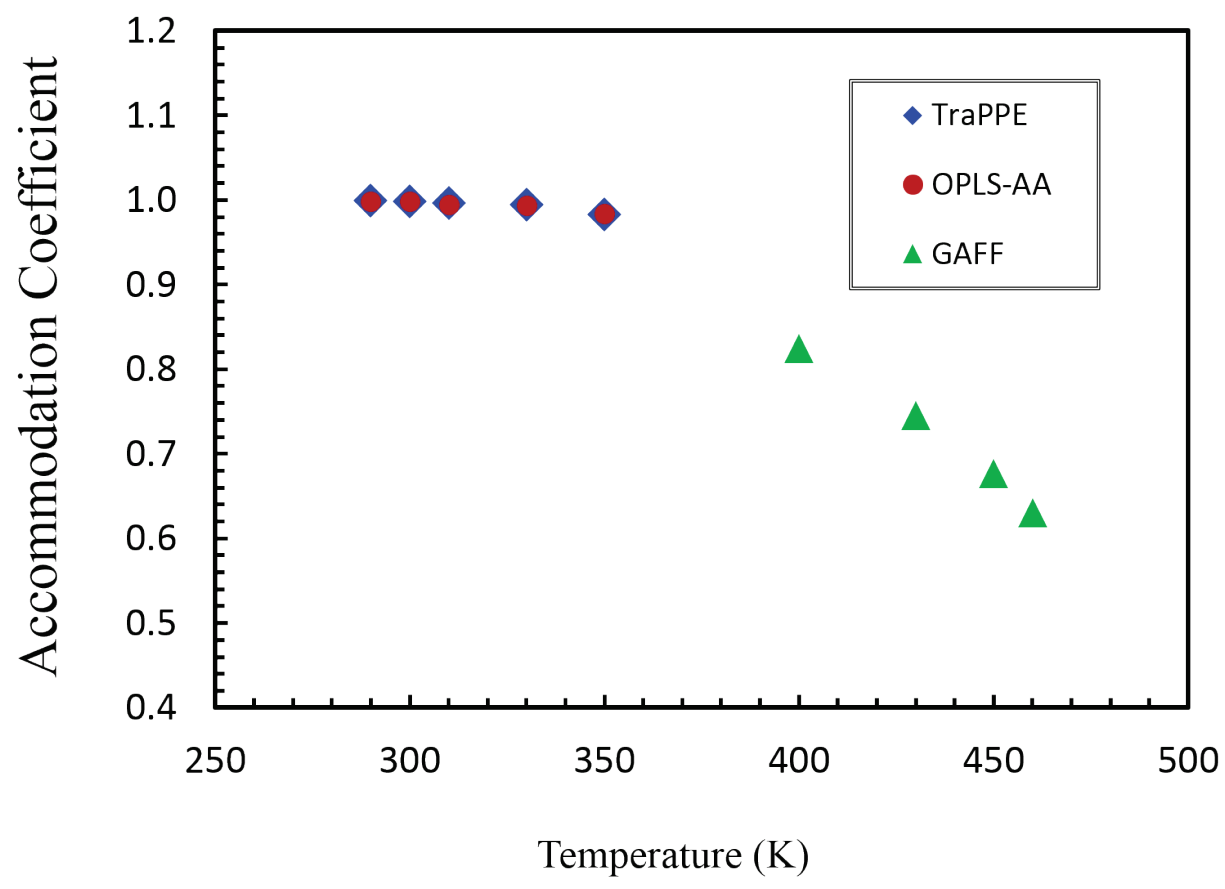

Figure 5-6: Macroscopic Accommodation Coefficient

(The accommodation coefficents were computed with the two octane molecular models, TraPPE and OPLS-AA. The literature values from Nagayama et al. [161] are shown labeled by the GAFF model used in their work.)

\subsection{Conclusions}

We have used extensive MD simulations to study the condensation probabilities of octane. A statistical estimate of the mass accommodation coefficient of octane at five liquid temperatures: $290 \mathrm{~K}, 300 \mathrm{~K}, 310 \mathrm{~K}, 330 \mathrm{~K}$, and $350 \mathrm{~K}$ was provided. The condensation probability of octane was observed to be consistently 0.99 at room temperature (290 to $310 \mathrm{~K}$ ) and decreasing at higher temperatures. The two octane molecular models, TraPPE and OPLS-AA, provided very close values for the condensation probability even though one was a neutral coarse-grained united atom

DISTRIBUTION STATEMENT A: Approved for public release. Distribution is unlimited. 
model and the other one was an all-atom model with partial charges, suggesting that in an estimate of the macroscopic condensation coefficient the use of a coarse-grained model is sufficient and justified by the computational savings. Our results are consistent with available data from other simulations and, for the first time, extend to lower temperatures where the condensation probability approaches unity. This work confirms that the accommodation coefficient of octane can be considered equal to 1.00 at and around room temperature. We have found that the accommodation coefficient decreases below the value $\left(\alpha_{c}=1\right)$ above $330 \mathrm{~K}$. For the first time, using atomicscale molecular dynamics simulations, an estimate of the evaporative flux of octane in vacuum was derived. 


\section{MODELING PHASE CHANGE MATERIAL IN MICRO-FOAM UNDER CONSTANT TEMPERATURE CONDITION}

\subsection{Introduction}

Metal or graphite foams filled with phase change materials (PCM) are attractive for thermal energy storage (TES) particularly in high heat flux applications as they provide both high storage capacity and short time constants. The latent heat of PCM provides high heat capacity whereas the foam structure increases the effective thermal conductivity. In earlier studies of phase change materials in foams, the volume-averaged (macroscopic) approach such as one-temperature and two-temperature models has been used, in which either local thermal equilibrium (LTE) between PCM and foams was assumed, or the simplified Newton's cooling law was applied $[189,190]$. The volume-averaged simulation can be effective for porous structure with simple geometry, however, this method can be restrictive for the modeling of foams with complex geometry. The complex foam structure which affects the effective thermal conductivity and interstitial heat transfer coefficient and subsequently the PCM melting/solidification needs to be accurately modeled to provide a better understanding of the heat transfer behavior of the system. The conventional method of effective thermal conductivity derived from packed bed with discrete solid particles as is done for simple porous structures cannot be applied here without modification and more accurate methods such as direct numerical simulation are required.

We investigate the phase change in a low porosity micro-foam using both volume-averaged and direct numerical simulation [191-193]. The foam was modeled as an ideal structure with BCC unit lattice and spherical micro-pores. The temperature at the top surface of the porous structure was kept constant. The objective of this work is to compare various simulation methodologies and identify one that can model the heat transfer process in PCM and micro-foam structure with sufficient efficiency and accuracy. The volume-averaged simulations were carried out using oneand two-temperature models, where as in the DNS, separate energy equations were applied to micro-foam and PCM domains and conjugate heat transfer was considered on the interface. Results including melted PCM volume fraction, temperature difference between PCM and micro-foam, instantaneous temperature profile, and heat flux are discussed.

\subsection{Model and Methods}

\subsubsection{Physical Model}

The physical model and geometry of the simulated micro-foam is shown in Figure 6-1. A cube with the side length of $2.56 \mathrm{~cm}$ was used for volume-averaged simulations (Figure 6-1a). The top surface was held at several constant temperatures $\left(T_{\text {top }}=334,351\right.$, or $\left.368 \mathrm{~K}\right)$, and the other surfaces were insulated. In direct numerical simulation (Figure 6-1b), the micro-foam geometry was composed of a series of unit cells and each was represented by a solid cube (side length of $400 \mu \mathrm{m}$ ) with nine spherical pores arranged in a BCC lattice [193-195]. The length in $Y$-direction was kept the same as that in the volume-averaged simulations and comprises of 64 unit cells. The $X$ and $Z$ lengths are much smaller $(0.02 \mathrm{~cm})$ to keep the mesh size and computation time at an affordable level. The top surface of the unit-cell array is specified with the same temperature condition as that in the volume-averaged simulations. The bottom surface of the unit-cell array was insulated. Symmetric conditions were specified at other surfaces. The initial temperature of $\mathrm{PCM} /$ micro-foam system was $300 \mathrm{~K}$ in both volume-averaged and direct simulations. 


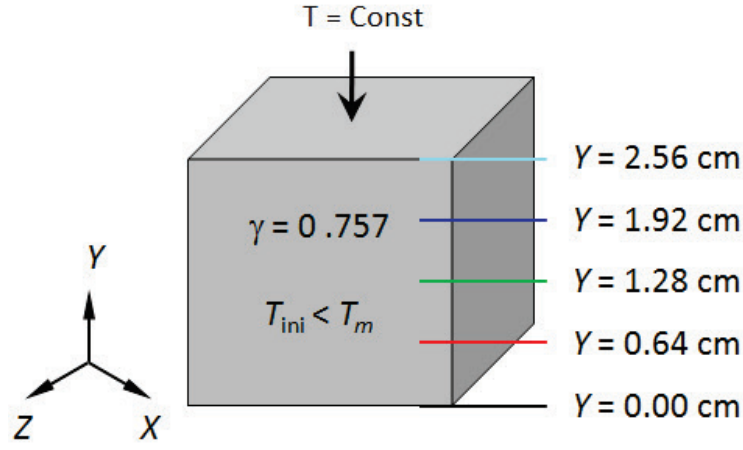

(a)
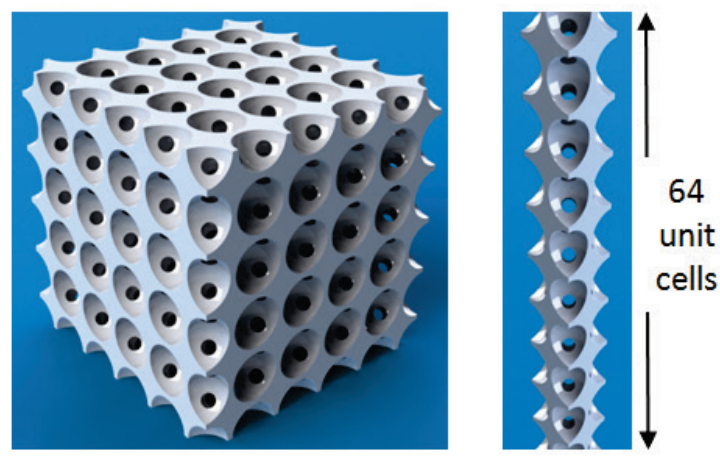

(b)

Figure 6-1: Geometry and Microstructure of the Simulated Micro-Foam

((a) Geometry and thermal boundary conditions in volume-averaged simulation; (b) Ideal foam microstructure (PCM not shown) in direct simulation. The top surface is held at a constant temperature.)

We considered aluminum foam and docosane (paraffin wax with $\mathrm{C}_{22} \mathrm{H}_{46}$ composition) PCM whose melting temperature of $317 \mathrm{~K}$ is below the top-wall temperature. It was assumed that the micro-foam is fully saturated with docosane and there is no volume change of PCM. All thermo-physical parameters of these two materials (Table 6-1) were kept constant in the simulations.

Table 6-1: Thermo-Physical Parameters for PCM and Micro-foam

\begin{tabular}{llllll}
\hline \hline & $\begin{array}{c}\rho \\
\left(\mathrm{kg} / \mathrm{m}^{3}\right)\end{array}$ & $\begin{array}{c}c_{p} \\
(\mathrm{~kJ} / \mathrm{kg}-\mathrm{K})\end{array}$ & $\begin{array}{l}k \\
(\mathrm{~W} / \mathrm{m}-\mathrm{K})\end{array}$ & $\begin{array}{l}L \\
(\mathrm{~kJ} / \mathrm{kg})\end{array}$ & $\begin{array}{l}T_{m} \\
(\mathrm{~K})\end{array}$ \\
\hline PCM & 785 & 2.89 & 0.4 & 260 & 317 \\
$\begin{array}{l}\text { Micro- } \\
\text { foam }\end{array}$ & 2719 & 8.71 & 202.4 & $\mathrm{~N} / \mathrm{A}$ & $\mathrm{N} / \mathrm{A}$ \\
\hline \hline
\end{tabular}

The foam porosity was 0.757 and pore size is $360 \mu \mathrm{m}$ for the current simualtions. The fluid flow within the melted PCM region can be neglected because such low porosity micro-foam provide a dominant resistance force to suppress the flow driven by the natural convection [196]. Thus, only the energy equations were solved in the simulations.

\subsubsection{Direct Numerical Simulation of PCM in Foam}

Without the need for extra ad hoc assumptions such as LTE, direct numerical simulations are considered as much closer to the real situation. In DNS, various energy equations were applied to PCM and the foam domains respectively, as shown in the following:

$\rho_{f} c_{p, f} \frac{\partial T_{f}}{\partial t}=\nabla \cdot\left(k_{f} \nabla T_{f}\right)-\rho_{f} L \frac{\partial f_{l}}{\partial t}$

$\rho_{s} c_{p, s} \frac{\partial T_{s}}{\partial t}=\nabla \cdot\left(k_{s} \nabla T_{s}\right)$ 
Conjugate heat transfer was considered at the PCM/micro-foam interface:

$$
\left.\begin{array}{c}
T_{f}=T_{s} \\
k_{f} \frac{\partial T_{f}}{\partial n}=k_{s} \frac{\partial T_{s}}{\partial n}
\end{array}\right\}
$$

Subscripts $f$ and $s$ in Eqs. (1-3) stand for PCM and foam respectively; $\rho, c_{p}, T, t, k$, and $L$ are density, specific heat, temperature, time, thermal conductivity and latent heat of fusion. In the right hand side of Eq. (6-1), $f_{l}$ is the volume fraction with the value of 1 in the melted PCM region, 0 in the solid PCM region, and between 0 and 1 in the mushy region. Effects of porosity and pore size have been directly included in the ideal foam geometry.

\subsubsection{One-temperature Simulation of Phase Change in Foam}

One-temperature model assumes that PCM and the micro-foam have the same temperature. Therefore, only one energy equation is applied for the whole system:

$\left(\gamma \rho_{f} c_{p, f}+(1-\gamma) \rho_{s} c_{p, s}\right) \frac{\partial T}{\partial t}=\nabla \cdot\left(k_{e f f} \nabla T\right)-\rho_{f} \gamma L \frac{\partial f_{l}}{\partial t}$

where $\gamma$ is the porosity, with value 1 standing for pure solid. The effective thermal conductivity $k_{e f f}$ is a function of porosity, thermal conductivity of each component, and more importantly, the detail structure of the foam. The simplest and commonly used expression $k_{e f f}=\gamma k_{f}+(1-\gamma) k_{s}$ is essentially the arithmetic mean. In PCM/micro-foam system, a more accurate $k_{e f f}$ can be obtained by experiments [197-199], or by DNS of real or ideal foam geometries [193-195, 200202]. In this paper, we have carried out DNS to find the effective thermal conductivity, which matches the Progelhoff model [15] $k_{e f f}=\gamma k_{f}+(1-\gamma)^{\alpha} k_{s}$ with $\alpha=1.31$ through the curve fitting, when pores in ideal foam structure is arranged in a BCC lattice [203].

\subsubsection{Two-temperature Simulation of Phase Change in Foam}

In two-temperature model, energy equations for PCM and foam need to be considered separately because of the local thermal non-equilibrium (LTNE). Their heat transfer is modeled by the Newton's law of cooling:

$$
\begin{aligned}
& \gamma \rho_{f} c_{p, f} \frac{\partial T_{f}}{\partial t}=\nabla \cdot\left(k_{f, e f f} \nabla T_{f}\right)-\rho_{f} \gamma L \frac{\partial f_{l}}{\partial t}-h_{V}\left(T_{f}-T_{s}\right) \\
& (1-\gamma) \rho_{s} c_{p, s} \frac{\partial T_{s}}{\partial t}=\nabla \cdot\left(k_{s, e f f} \nabla T_{s}\right)-h_{V}\left(T_{s}-T_{f}\right)
\end{aligned}
$$

where effective thermal conductivities for PCM $k_{f, \text { eff }}$ and for the foam $k_{s, \text { eff }}$ are still related to foam structure. Here we used two sets of effective thermal conductivities: one corresponds to the arithmetic mean such that $k_{f, e f f}=\gamma k_{f}$ and $k_{s, e f f}=(1-\gamma) k_{s}$. The other corresponds to the Progelhoff model, with $k_{f, e f f}=\gamma k_{f}$ and $k_{s, e f f}=(1-\gamma)^{\alpha} k_{s}$ respectively. The volumetric heat transfer coefficient $h_{V}=A_{f s} h_{f s}$, where the specific surface area $A_{f s}$ is the ratio of interstitial surface area to the representative elementary volume (REV), and $h_{f s}$ is the interstitial heat transfer coefficient between PCM and foam. Both $h_{f s}$ and $A_{f s}$ are highly dependent on the foam structure. In this work, the specific surface area $A_{f s}=1.08 \times 10^{4} \mathrm{~m}^{-1}$ is obtained from the foam structure 
described in Section 6.2.1. The interstitial heat transfer coefficient is defined as $h_{f s}=q_{f s}^{\prime \prime} /\left(\bar{T}_{s}-\right.$ $\bar{T}_{f}$ ), where $q_{f s}^{\prime \prime}$ is the heat flux at the fluid/foam interface, $\bar{T}_{s}$ is the volume-weighted average temperature for micro-foam and $\bar{T}_{f}$ is the mass-weighted average temperature for fluid. We applied direct simulation on the unit-cell level, and based on the calculated heat flux across the interface and the average PCM and foam temperatures we obtained the value of interstitial heat transfer coefficient $h_{f s}=9.09 \times 10^{3} \mathrm{~W} /\left(\mathrm{m}^{2} \mathrm{~K}\right)$.

\subsubsection{Problem Setup}

For these simulations, the commercial CFD package ANSYS FLUENT 13.0 was used with double precision. Parallel and batch processing were carried in the AFRL DoD Supercomputer Resource Center (DSRC). To apply the two-temperature model in FLUENT, a user-defined scalar (UDS) was used for micro-foam temperature. The convergence criterion of absolute scaled residual for energy equation was set to be $10^{-9}$ for volume-averaged simulations and $10^{-11}$ for DNS. Grid- and timestep-independent results were ensured first. We found that the grid with 90000 cells and time step size $d t=0.02 \mathrm{~s}$ are enough for one- and two-temperature simulations, while we used the grid with 9 million cells and $d t=0.008 \mathrm{~s}$ for direct simulations. A typical DNS needed over 30,000 CPU hours (i.e., $192 \mathrm{CPU}$ cores for around 160 hours), much more expensive than volume-averaged simulations.

\subsection{Result and Discussion}

The direct numerical simulations show very complicated micro-scale evolutions of melting front and temperature distributions, which are very different from the one-dimensional macroscale behavior distributions generated by the volume-averaged simulations. In order to compare different simulation techniques, we studied heat transfer behavior such as the volume fraction of melted PCM, average temperature at different locations, temperature difference between microfoam and PCM, and the top-wall heat fluxes.

\subsubsection{Volume Fraction of Melted PCM}

Figure 6-2 shows the profiles of melted PCM volume fraction with top surface temperature fixed at $334 \mathrm{~K}$ and $368 \mathrm{~K}$, respectively. Here "1-T" and "2-T" stand for one- and two-temperature simulations. "Arithmetic Mean" and "Progelhoff" refer to the arithmetic mean and Progelhoff models for the effective thermal conductivity. First, we can see that one- and two-temperature simulations with the arithmetic mean predict higher melting rate than those from Progelhoff model and the DNS. This is because the arithmetic mean generates higher effective thermal conductivity, thus enables system absorbing more heat to melt PCM quickly. Second, one-temperature and twotemperature simulations accurately predict volume fraction of melted PCM, when the Progelhoff model is used for the effective thermal conductivity $k_{e f f}$. Hence, modeling of $k_{e f f}$ plays a key role in the volume-averaged simulation. As prescribed by Progelhoff model, $k_{e f f}$ nonlinearly depends on the porosity, and is dominated by the thermal conductivity of micro-foam. As the enforced top surface temperature is raised (Figure 6-2b), the temperature gradient at the top surface increases and induces an earlier melting of PCM. 


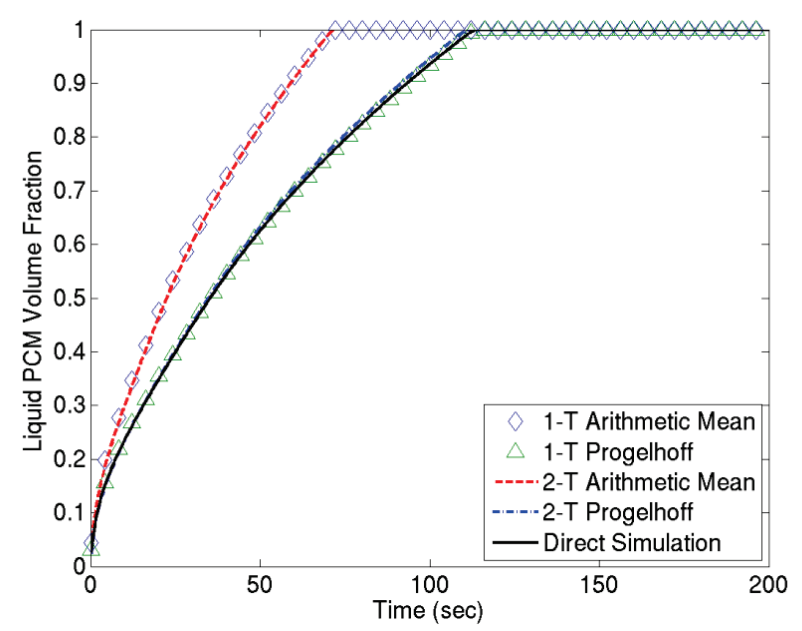

(a)

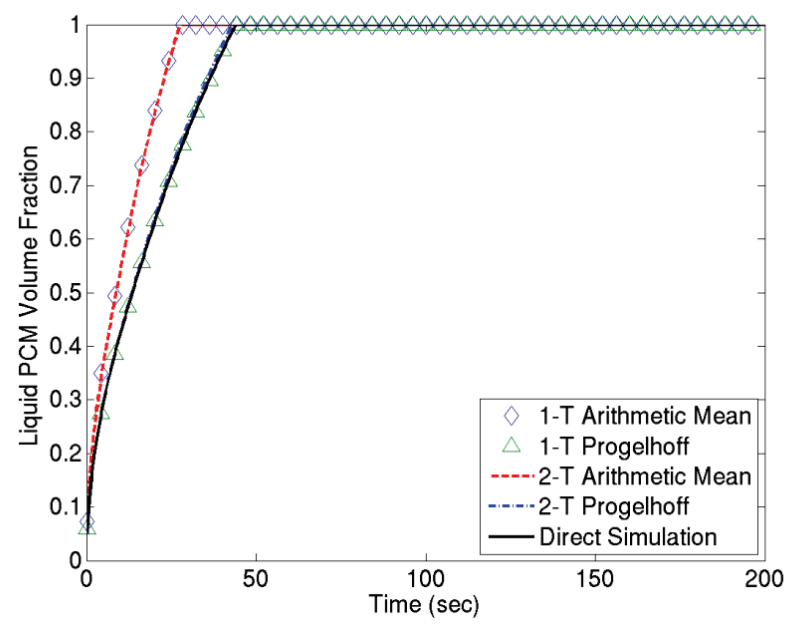

(b)

Figure 6-2: Profiles of Melted PCM Volume Fraction (a) $T_{\text {top }}=334 \mathrm{~K}$; (b) $T_{\text {top }}=368 \mathrm{~K}$

\subsubsection{Average Temperature at Different $Y$ Cross Sections}

Figure 6-3 shows the time history of area-weighted average temperature at two cross sections in the foam, with the top-surface temperature fixed at $334 \mathrm{~K}$. At a cross section close to the top surface (Figure 6-3a), the temperature simulated from volume-averaged method and Progelhoff model reasonably fit the results from DNS. Arithmetic model of effective thermal conductivity, however, seemingly over-estimates the temperature change by $12 \%$ in the phase change region compared to DNS.

At the bottom of the system (Figure 6-3b), this over-estimation gets worse. When the effective thermal conductivity is calculated from arithmetic mean, the volume-averaged simulation predicts earlier starting time of phase change compared to direct simulation (Figure 6-3b), indicating the over-estimation of the effective thermal conductivity of PCM/foam system by arithmetic mean. When the Progelhoff model is applied to calculate $k_{e f f}$, the temperature variation is well captured, no matter whether one-temperature or two-temperature simulation is conducted. It is noteworthy that there is a temperature plateau at the structure bottom (Figure 6-3b), in which heat transfer is primarily dominated by phase change. In contrast, the average temperature

DISTRIBUTION STATEMENT A: Approved for public release. Distribution is unlimited. 
at the cross section near the top surface continuously increases, even during the phase change process (Figure 6-3a), indicating the fast rising temperature of foam structure at this cross section due to its high conductivity. As the cross section moves toward the bottom, the heat conduction through foam structure slows down, and the phase change process is more pronounced.

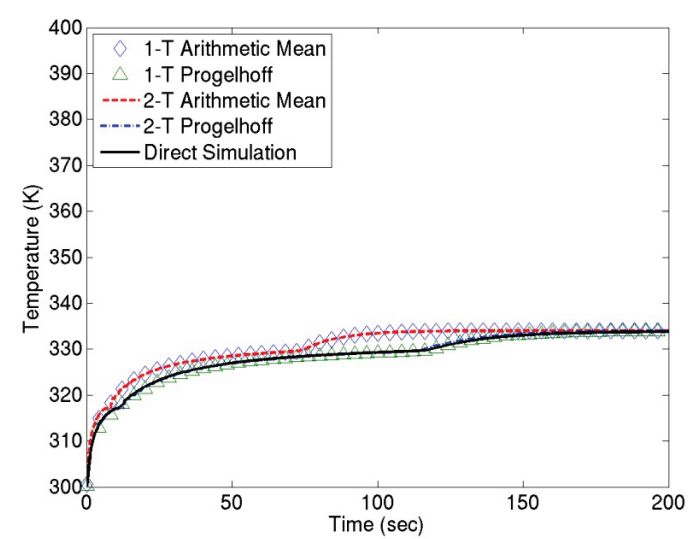

(a)

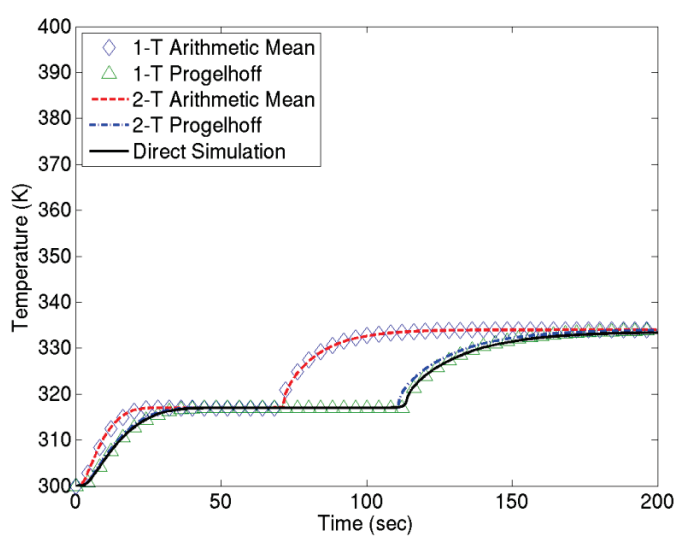

(b)

Figure 6-3: Average Temperature Profiles

(At (a) cross section $Y=1.92 \mathrm{~cm}$, and (b) bottom plane $Y=0.0 \mathrm{~cm}$. The top-surface temperature $T_{\text {top }}=$ $334 \mathrm{~K}$.)

\subsubsection{Temperature Difference between Micro-foam and PCM}

In two-temperature and direct numerical simulations, the effective thermal conductivity and the interstitial heat transfer are two competing factors affecting the thermal process between micro-foam and PCM. Larger differences in values of effective thermal conductivities between micro-foam and PCM induces larger temperature difference between them; while a stronger interstitial heat transfer makes the temperature jump smaller. Comparison of temperature difference cross the micro-foam and PCM interface $\delta T=T_{s}-T_{f}$ will help us better evaluate the two-temperature simulation.

The temperature differences at two cross sections $(Y=1.92 \mathrm{~cm}$ and $0.0 \mathrm{~cm})$ from twotemperature and direct simulations are shown in Figure 6-4. The onset of phase change (melting) is indicated by the peak, which separates pre- and post-melting stages. With results from direct simulations as the reference, we can see that the two-temperature simulation using arithmetic mean model predicts earlier melting stages in both $Y$-locations, and the peak magnitude is dramatically over-estimated at the cross section close to the top surface $(Y=1.92 \mathrm{~cm})$. However, twotemperature simulations using Progelhoff model predicts acceptable time onset of melting process and the temperature difference. With the cross section further away from the top-wall (e.g., at $Y=$ $0.0 \mathrm{~cm}$ ), the discrepancy in the melting onset is more obvious.

Temperature difference under a higher top-wall temperature $\left(T_{\text {top }}=368 \mathrm{~K}\right)$ is presented in Figure 6-5. The melting onset is advanced as the top-wall temperature is increased. We can see that two-temperature simulation with Progelhoff model predicts nearly the same starting time of phase change as that from direct simulation in both cross sections. 


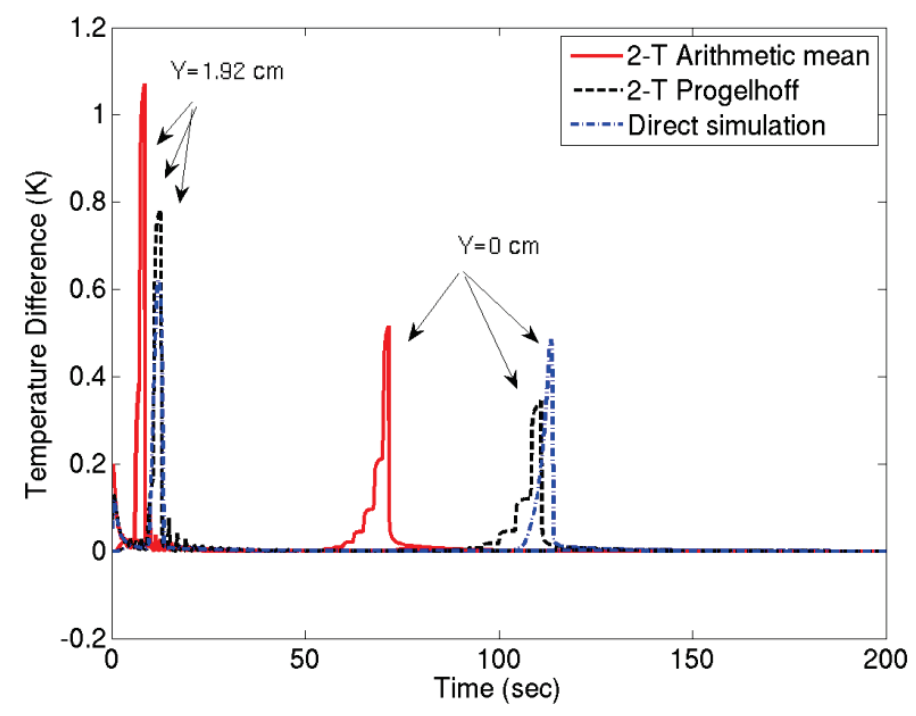

Figure 6-4: Comparison of Temperature Difference between Micro-foam and PCM ( $\mathrm{T}_{\text {top }}=$ $334 \mathrm{~K})$

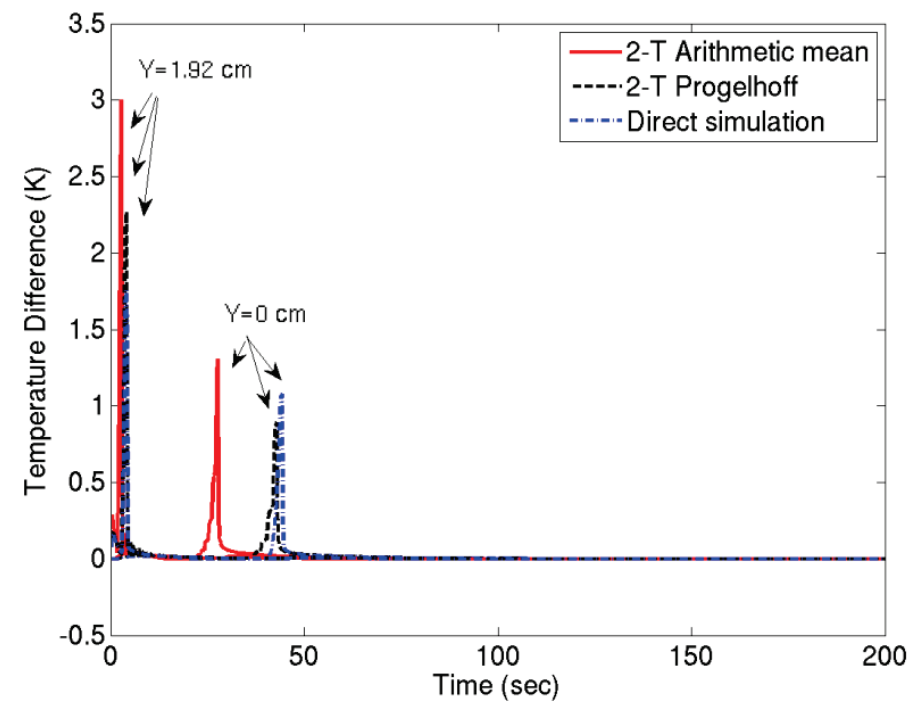

Figure 6-5: Comparison of Temperature Difference between Micro-foam and PCM ( $T_{\text {top }}=$ $368 \mathrm{~K})$

\subsubsection{Heat Flux at Top Surface}

We further calculated the area-weighted average heat flux at top surface using various simulation methodologies. In one-temperature simulation, only the overall top-wall heat flux can be calculated. In two-temperature simulation and DNS, we can calculate the top-wall heat flux for each component, i.e., the amount of heat flows through the PCM and through micro-foam respectively. The overall heat flux at the top surface is just the sum of heat flux from PCM and the foam, i.e., $q_{t o p}^{\prime \prime}=s q_{f, t o p}^{\prime \prime}+(1-s) q_{s, t o p}^{\prime \prime}$, where $s$ is the area ratio of PCM surface area to the total on the top surface. The heat flux of the foam is defined as $q_{s, t o p}^{\prime \prime}=\frac{k_{e f f, s}\left(T_{s, \text { cell@top }}-T_{t o p}\right)}{r_{d}}$, in which 
$T_{s, \text { cell@top }}$ is the foam temperature at the centroid of a cell near the top surface, and $r_{d}$ is the distance from the cell centroid to the top surface. In the current study, both the overall and individual heat fluxes at the top surface are normalized by the upper limit heat flux, which is defined as $q_{\text {max }}^{\prime \prime}=H\left\{\gamma \rho_{f} L+\left(\gamma \rho_{f} c_{p, f}+(1-\gamma) \rho_{s} c_{p, s}\right)\left(T_{\text {top }}-T_{\text {ini }}\right)\right\} . H$ is the distance from the top to the bottom surface, and is the cube length in our case.

Figure 6-6 (a) shows the normalized overall heat flux at top surface. Volume-averaged simulations predict the heat flux reasonably well. However, the effective thermal conductivity calculated from Progelhoff model generates more accurate results than from arithmetic mean model (Figure 6-6 (a) insert). The heat flux of individual material components is presented in Figure 6-6 (b). From the insert, it can be seen that the heat flux from the micro-foam is much larger than that from PCM, since the thermal conductivity of the micro-foam is three orders of magnitude higher than that of PCM. (Table 6-1). 
(a)

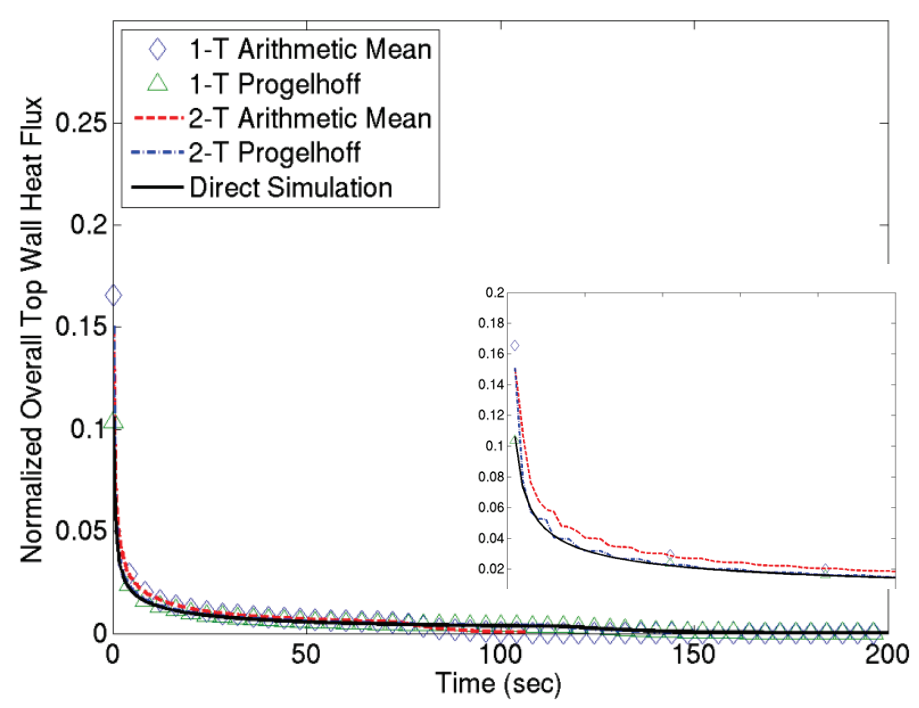

(b)

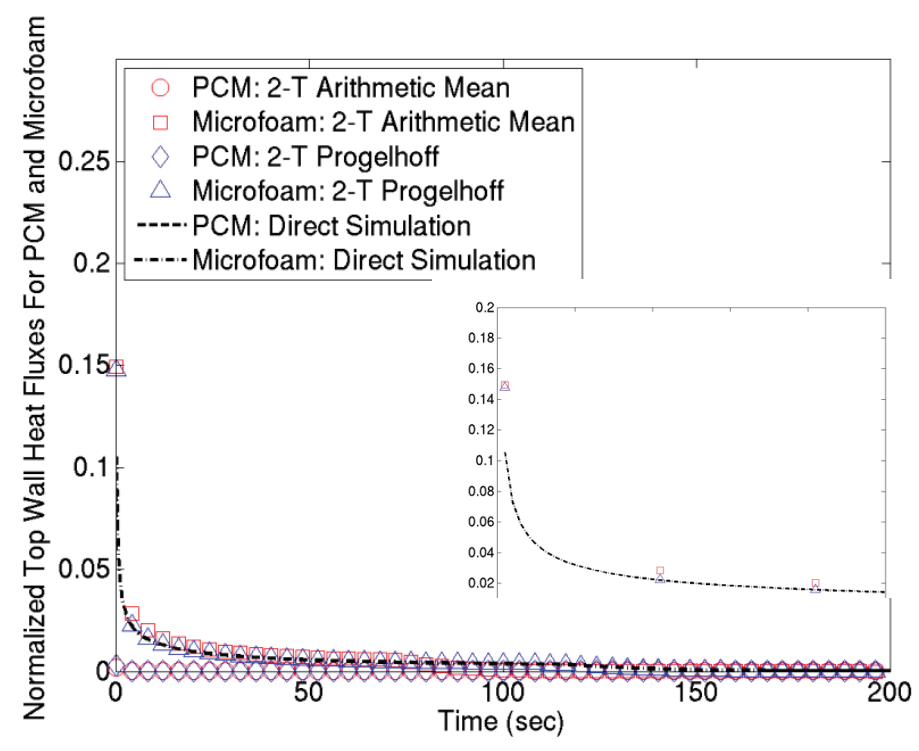

Figure 6-6: Heat Flux at Top Surface

((a) Overall; (b) Individual Component; $\boldsymbol{T}_{\text {top }}=334 \mathrm{~K}$ )

We also calculated the overall and individual heat fluxes on the top surface, at which the temperature $T_{\text {top }}$ is raised to $368 \mathrm{~K}$ (Figure 6-7). The flux is increased at the top surface, contributed principally by the micro-foam. It is worth mentioning that, unlike the temperature at various cross sections, the heat flux gradually and slowly approaches a steady value. This can be ascribed to the varying effective thermal conductivity and temperature gradient in the system. 
(a)

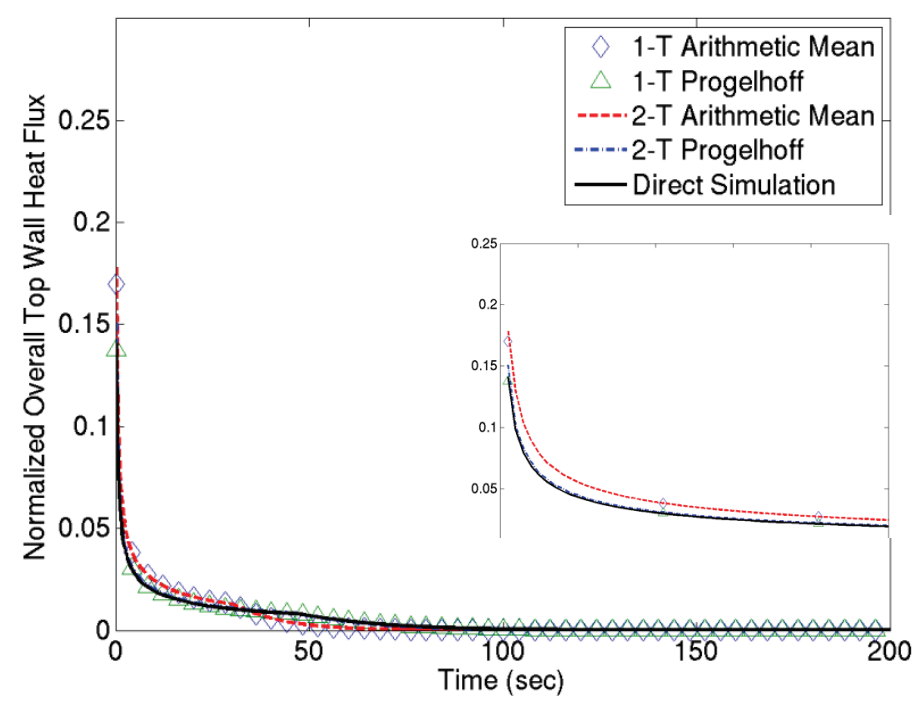

(b)

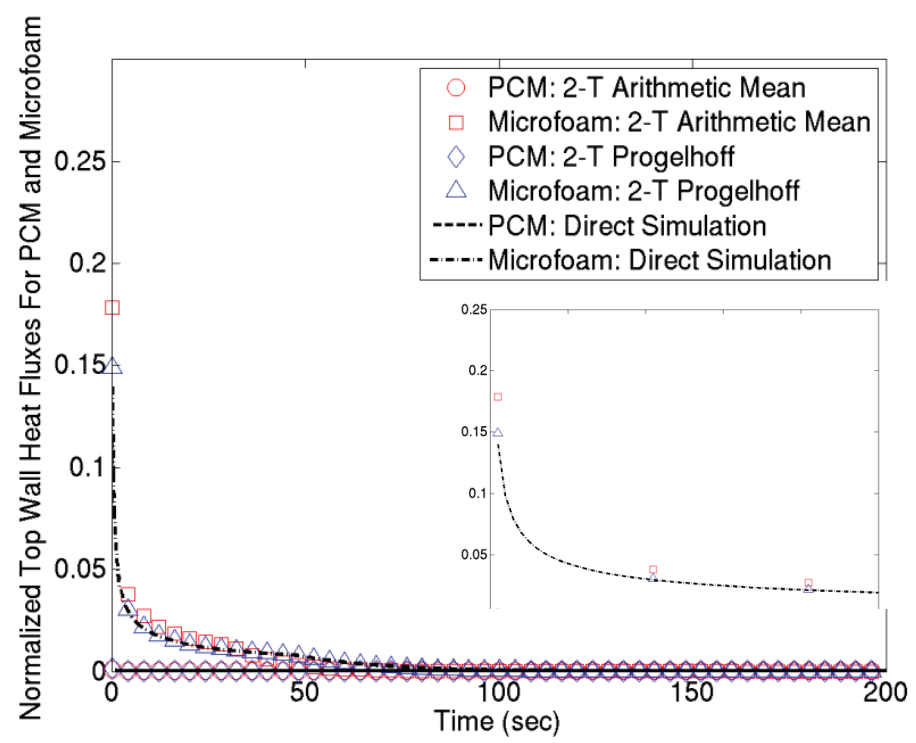

Figure 6-7: Heat Flux at Top Surface (a) Overall (b) Individual Component $\left(T_{\text {top }}=368 \mathrm{~K}\right)$

\subsection{Conclusions}

In this work, we numerically studied the heat transfer behavior of a phase change material in an open-cell micro-foam using both volume-averaged and direct simulations. Heat transfer process was simulated for a spherical-pore embedded micro-foam, and its top surface was kept isothermal. By examining the volume fraction of melted PCM, average temperature at different locations, temperature jump across micro-foam and PCM interface, and average heat flux at the top surface, we find that volume-averaged simulations can reasonably predict the heat transfer process in the micro-foam with complex geometry, as long as accurate thermo-physical parameters such as effective thermal conductivity are provided. In the present study, the effective thermal conductivity of the PCM/micro-foam system was obtained from direct numerical simulation of a unit cell. The simulations results supported the Progelhoff model of effective thermal conductivity

DISTRIBUTION STATEMENT A: Approved for public release. Distribution is unlimited. 
where the effective thermal conductivity can be expressed as a power law of porosity and a power factor of 1.3 was calculated for the model structure. We found that the heat flux near the enforced isothermal boundary is dominated by the micro-foam due to its high thermal conductivity. The phase change heat transfer becomes gradually more pronounced in locations away from the enforced high temperature boundary. 


\section{NUMERICAL MODELING OF HEAT TRANSFER IN OPEN-CELL MICRO-FAM WITH PHASE CHANGE MATERIAL}

\subsection{Introduction}

Paraffin wax-based PCM have been widely used in TES devices. Their thermal properties such as suitable melting temperatures, good storage densities, high specific heats, little or no subcooling, and good chemical, thermal and cycling stabilities [204] make them valuable for use in these devices. However, one primary drawback of these PCMs is their low thermal conductivities. To increase the effective thermal conductivities, several thermal conductivity enhancers (TCE) such as fin or partition [205-209], packed particles [210], micro- or macro-encapsulation [211, 212], and metal [213] or graphite foams [214] are used. Of these various PCM/TCE approaches, PCM/foam systems show great potential for use in TES devices particularly those requiring fast thermal transient response [112]. The foam helps to transport the heat from the source to the PCM and its large interfacial surface area distributes the heat more effectively in the PCM. The complex microstructure of the foam however leads to complicated heat transfer properties and many experimental studies have been devoted to obtaining their effective thermo-physical properties of fluid/foam systems. Paek et al. [198] investigated aluminum foams with different porosities and cell sizes and found that while the effective thermal conductivity is mainly affected by the porosity, the permeability is determined by both porosity and cell size. Hong and Herling $[215,216]$ studied PCM saturated open-cell metal foam and found that the specific surface area (or surface area density) directly affects the system cooling time. They also observed that both porosity and cell size have strong influences on the thermal transport behavior. Calmidi and Mahajan [197] measured the effective thermal conductivity of metal foams filled with air and water. Their experimental results match well with those from theoretical modeling of ideal 2D hexagonal foams. Similar work has also done by Bhattacharya et al. [199] for more complicated foam structures.

Numerical simulation studies have considered the microstructure to varying degrees and have provided great insight into the influence of porosity and cell size on thermal properties. However, these studies have been mainly limited to single phase flows. Druma et al. used the finite element method to calculated the effective thermal conductivity of an ideal foam with periodic unit cells and body-centered-cubic (BCC) pore distribution [193]. Their simulation results matched the theoretical prediction [217] at low porosity levels, but significant differences were observed at high porosity levels. Maruyama et al. [200] calculated the effective thermal conductivity of carbon foam based on 3D rendered foam geometry. Krishnan et al. [194, 218] used different ideal foam structures filled with air and water to calculate the effective thermal conductivity, permeability and interstitial heat transfer coefficient, and their results agree well with available experimental data [219]. Bodla et al. [201] calculated the thermo-physical parameters of a metal foam with a rendered geometry from micro-computed tomography ( $\mu-\mathrm{CT})$. They also proposed a resistance network model for faster computation of effective thermal conductivity by employing an analogy between electrical and thermal conduction [202].

Unlike single-phase flow, the study of phase change in foams has only recently received attention. Majority of the simulations have been done using idealized volume average models. Volume-averaged simulations based on one-temperature model [189, 220] using the LTE assumption have found the results to be inaccurate, for cases where the difference of thermal conductivity between the foam and PCM is very large or when PCM is under rapid heating or cooling especially for small Sparrow numbers [221]. Subsequently, volume-averaged twotemperature model with local thermal non-equilibrium (LTNE) assumption has been utilized.

DISTRIBUTION STATEMENT A: Approved for public release. Distribution is unlimited. 
Krishnan et al. [222, 223] carried out a parametric study of phase change with natural convection in metal foams under constant or pulsed heating and discussed the behavior of melt front evolution and melting rate. Other two-temperature simulations on phase change in metal foam have also been carried out [190, 224, 225]. These studies are all based on volume-average methods that do not fully represent the effects of porosity and cell size. As previously discussed, both of these effects have been found to have significant influences on the effective thermal conductivities and interstitial heat transfer coefficient, which in turn affect the heat transfer characteristics.

In order to better understand the effect of the foam microstructure on PCM phase change behavior, we have used DNS and compared with results from volume average studies. Our previous preliminary study [192] under constant temperature conditions found that the heat transfer prediction using volume average methods can be improved by using thermo-physical parameter such as effective thermal conductivity from DNS. In the current study we extend the investigation to constant heat flux conditions. Thermo-physical properties were extracted from DNS simulations. These were then used in volume-averaged one-temperature (LTE assumption) and two-temperature (NLTE assumption) simulations. The effectiveness of these models in phase change modeling in micro-foam was evaluated.

\subsection{Methods and Models}

The fixed-grid enthalpy-porosity method [226] was used to simulate the solid-liquid phase change process in the PCM. A description of DNS, one-temperature model based on LTE assumption, and two-temperature model based on LTNE assumption is provided below.

\subsubsection{Direct Numerical Simulation}

Two sets of governing equations are applied to the PCM and the micro-foam. Conjugate heat transfer is considered at the PCM/micro-foam interface and it is assumed that there is no contact resistance or heat source/sink. The control equations and interface thermal boundary conditions can be expressed as:

$$
\begin{aligned}
& \frac{\partial \rho_{f}}{\partial t}+\nabla \cdot\left(\rho_{f} \boldsymbol{u}_{f}\right)=0 \\
& \frac{\partial\left(\rho_{f} \boldsymbol{u}_{f}\right)}{\partial t}+\nabla \cdot\left(\rho_{f} \boldsymbol{u}_{f} \boldsymbol{u}_{f}\right)=-\nabla p_{f}+\nabla \cdot\left(\mu_{f} \nabla \boldsymbol{u}_{f}\right)+\boldsymbol{f}_{b}+\boldsymbol{S}_{u} \\
& \rho_{f} c_{p, f}\left(\frac{\partial T_{f}}{\partial t}+\boldsymbol{u}_{f} \cdot \nabla T_{f}\right)=\nabla \cdot\left(k_{f} \nabla T_{f}\right)-\rho_{f} L \frac{\partial f_{l}}{\partial t} \\
& \rho_{s} c_{p, s} \frac{\partial T_{s}}{\partial t}=\nabla \cdot\left(k_{s} \nabla T_{s}\right) \\
& \left\{\begin{array}{l}
T_{f}=T_{s} \\
k_{f} \nabla T_{f} \cdot \boldsymbol{n}=k_{s} \nabla T_{s} \cdot \boldsymbol{n} \quad \text { at the PCM/foam interface }
\end{array}\right.
\end{aligned}
$$

where, subscripts $f$ and $s$ stand for PCM and solid foam, respectively. $\rho, t, \boldsymbol{u}, p, \mu, \boldsymbol{f}_{b}, c_{p}, T, k, L$, and $\boldsymbol{n}$ are density, time, velocity, pressure, dynamic viscosity, body force, specific heat capacity, temperature, thermal conductivity, latent heat of fusion, and normal unit vector, respectively. The momentum source term in Eq. (7-2) is calculated using the Kozeny-Carman relationship $\boldsymbol{S}_{\boldsymbol{u}}=$ $-A \frac{\left(1-f_{l}\right)^{2}}{f_{l}{ }^{3}} \boldsymbol{u}_{f}$ with a large coefficient $A=10^{5} \sim 10^{6}$. The liquid volume fraction $f_{l}$ in Eq. (7-3) is 1 in 
liquid PCM, 0 in solid PCM, and between 0 and 1 in the mushy region [226]. The foam structure is a series of unit cells, with each represented by a solid cube with nine spherical pores arranged in a BCC lattice $[193,194,218]$.

\subsubsection{One-temperature Simulation}

In the one-temperature volume-averaged method, the foam is simply treated as a porous medium, and the momentum equation are modified accordingly as:

$$
\frac{\partial\left(\rho_{f} \boldsymbol{u}_{f}\right)}{\partial t}+\nabla \cdot\left(\rho_{f} \boldsymbol{u}_{f} \boldsymbol{u}_{f}\right)=-\nabla p_{f}+\nabla \cdot\left(\mu_{f} \nabla \boldsymbol{u}_{f}\right)+\boldsymbol{f}_{b}+\boldsymbol{S}_{u}+\boldsymbol{F}_{R}
$$

in which the resistant force $\boldsymbol{F}_{R}$ is described by the Forchheimer's extension of Darcy's law [227]:

$$
\boldsymbol{F}_{R}=-\frac{\mu_{f}}{K} \boldsymbol{u}_{f}-\frac{\rho_{f} F}{\sqrt{K}}\left|\boldsymbol{u}_{f}\right| \boldsymbol{u}_{f}
$$

The permeability $K$ and the inertial coefficient $F$ can be determined from the porosity and cell size of the foam structure, through either experiments [213] or DNS [194, 218]. Additional discussion is provided in Section 7.2.4.

Assuming that there is no temperature difference between the PCM and the foam, the onetemperature energy equation can be written as:

$$
\left(\gamma \rho_{f} c_{p, f}+(1-\gamma) \rho_{s} c_{p, s}\right) \frac{\partial T}{\partial t}+\gamma \rho_{f} c_{p, f} \boldsymbol{u}_{f} \cdot \nabla T=\nabla \cdot\left(k_{e f f} \nabla T\right)-\rho_{f} \gamma L \frac{\partial f_{l}}{\partial t}
$$

in which the effective thermal conductivity $k_{e f f}$ is a function of porosity $\gamma$, the thermal conductivity of each component, and the foam structure. $k_{e f f}$ can be simply calculated using arithmetic mean, i.e., $k_{e f f}=\gamma k_{f}+(1-\gamma) k_{s}$, or more accurate values can be extracted from experiments [197-199] or DNS [193, 194, 200-202, 218].

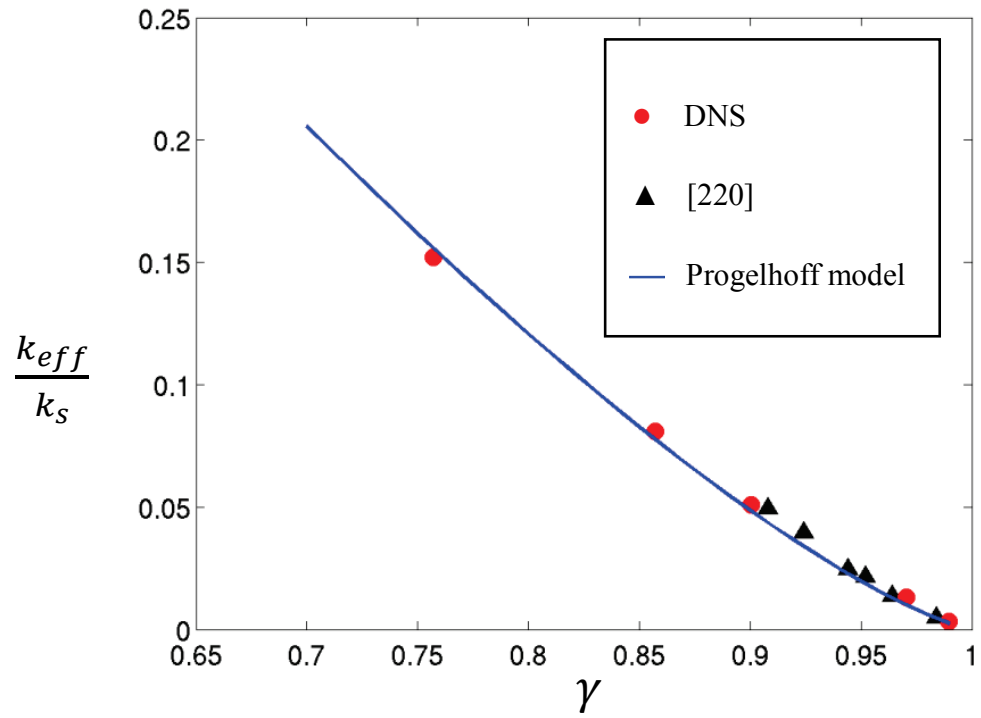

Figure 7-1: Effective Thermal Conductivity as a Function of Porosity

(The nonlinear relationship is well fitted by Progelhoff model.)

DISTRIBUTION STATEMENT A: Approved for public release. Distribution is unlimited. 
We used DNS to obtain the effective thermal conductivity of a fluid/foam system using a similar approach as that used by Druma et al. [193]. Aluminum foam with a micro- structure consisting of a periodic unit cell array was used. Five cell sizes with pore diameters 360, 380, 390, $410,420 \mu \mathrm{m}$ and corresponding porosities $0.7570,0.8570,0.9006,0.9704$, and 0.9898 , were considered. The extracted effective thermal conductivity is shown as a function of porosity in Figure 7-1. It can be seen that our results agree well with the experimental results from Krishnan et al. [194]. The calculated $k_{e f f}$ was found not to be a linear function of $\gamma$ and therefore instead of using the arithmetic mean, the Progelhoff model $k_{e f f}=\gamma k_{f}+(1-\gamma)^{\alpha} k_{s}$ was used to fit the data and the parameter $\alpha$ was determined to be 1.3142. The Progelhoff model which allows for inclusion of information regarding the foam microstructure was found to be more accurate than the widely used arithmetic mean.

\subsubsection{Two-temperature Simulation}

In the two-temperature model, a local temperature difference between PCM and the microfoam is considered. Energy equations for the PCM and the foam are considered separately. The heat transfer between the PCM and foam is modeled by the Newton's law of cooling:

$$
\begin{aligned}
& \gamma \rho_{f} c_{p, f}\left(\frac{\partial T_{f}}{\partial t}+\boldsymbol{u}_{f} \cdot \nabla T_{f}\right)=\nabla \cdot\left(k_{f, e f f} \nabla T_{f}\right)-\rho_{f} \gamma L \frac{\partial f_{l}}{\partial t}-h_{V}\left(T_{f}-T_{s}\right) \\
& (1-\gamma) \rho_{s} c_{p, s} \frac{\partial T_{s}}{\partial t}=\nabla \cdot\left(k_{s, e f f} \nabla T_{s}\right)-h_{V}\left(T_{s}-T_{f}\right)
\end{aligned}
$$

The effective thermal conductivity was calculated using both arithmetic mean and Progelhoff model with $\alpha=1.3142$. The volumetric heat transfer coefficient $h_{V}$ was expressed as $h_{V}=A_{f s} h_{f s}$, where $A_{f s}$ is the specific surface area (the ratio of interstitial surface area to the representative elementary volume), and $h_{f s}$ is the interstitial heat transfer coefficient between PCM and foam. Both $h_{f s}$ and $A_{f s}$ are foam structure dependent. In this study, $A_{f s}=10802 \mathrm{~m}^{-1}$ and was obtained for $\gamma=0.757$, from the ideal foam structure with unit cell with BCC pore distribution.

One of the principal challenges in the two-temperature model is determining $h_{f s}$ or the local Nusselt number $N u_{d}=h_{f s} d_{p} / k_{f}$ based on the cell-size $\left(d_{p}\right)$. Various analytical and empirical formulae have been developed to correlate $N u_{d}$ with porosity, local Reynolds number $R e_{d}$, Prandtl number $P r$, and Rayleigh number when natural convection dominates [222]. We have calculated $h_{f s}$ for water flowing through aluminum foam using an approach similar to earlier studies [194, 218]. Varying mass flow rates and pressure drops were considered and periodic velocity boundary condition was applied. We also varied the heat fluxes on the top and bottom foam surfaces. The interstitial heat transfer coefficient $h_{f s}$ can be expressed as $h_{f s}=q_{f s}^{\prime \prime} /\left(\bar{T}_{s}-\bar{T}_{f}\right)$, where $q_{f s}^{\prime \prime}$ is the heat flux at the fluid/foam interface, $\bar{T}_{s}$ is the volume-weighted average temperature for micro-foam, and $\bar{T}_{f}$ is the average temperature for fluid. The relationship between local Nusselt and Peclet numbers $P e_{d}=R e_{d} P r$ is shown in Figure 7-2. We see nonzero values of local Nusselt number at zero velocity due to the consideration of conjugate heat transfer between micro-foam and fluid in the conduction limit, which is different from earlier studies [194]. We calculated the interstitial heat transfer coefficient $h_{f s}=9.09 \times 10^{3} \mathrm{~W} /\left(\mathrm{m}^{2} \mathrm{~K}\right)$ for $\gamma=0.757$. It should be noted that this correlation between Nusselt number and interstitial heat transfer coefficient in two-temperature models is valid primarily in the steady state. 


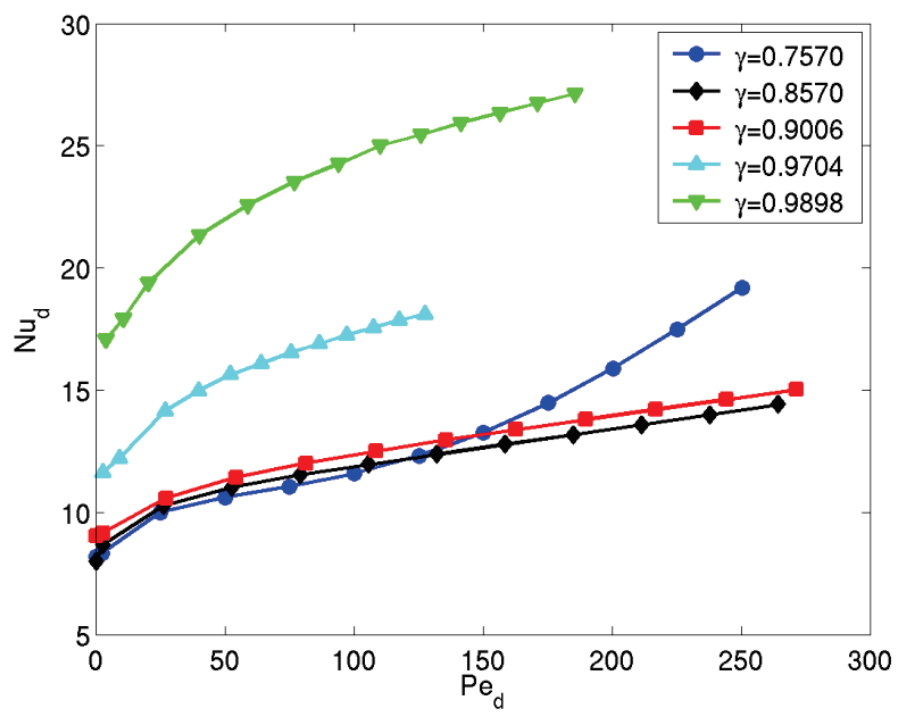

Figure 7-2: Relationship between the Local Nusselt and Peclet Numbers

(The local Nusselt number increases with local Peclet number for micro-foam, with porosity as a parameter.)

\subsubsection{Effect of natural convection in low-porosity micro-foam}

Effect of natural convection on heat transfer has been experimentally and numerically investigated for PCM/packed-bed system [210]. However, this effect might be suppressed in $\mathrm{PCM}$ /foam system due to the high resistant forces (Eq. (7)) provided by the micro-foam structure. To evaluate this resistance in micro-foams, we carried out DNS to calculate pressure drops of steady-state flows passing through micro-foams of different porosities [194]. Figure 7-3 shows that the pressure-drop rises with increased averaged inlet velocity and decreased micro-foam porosity.

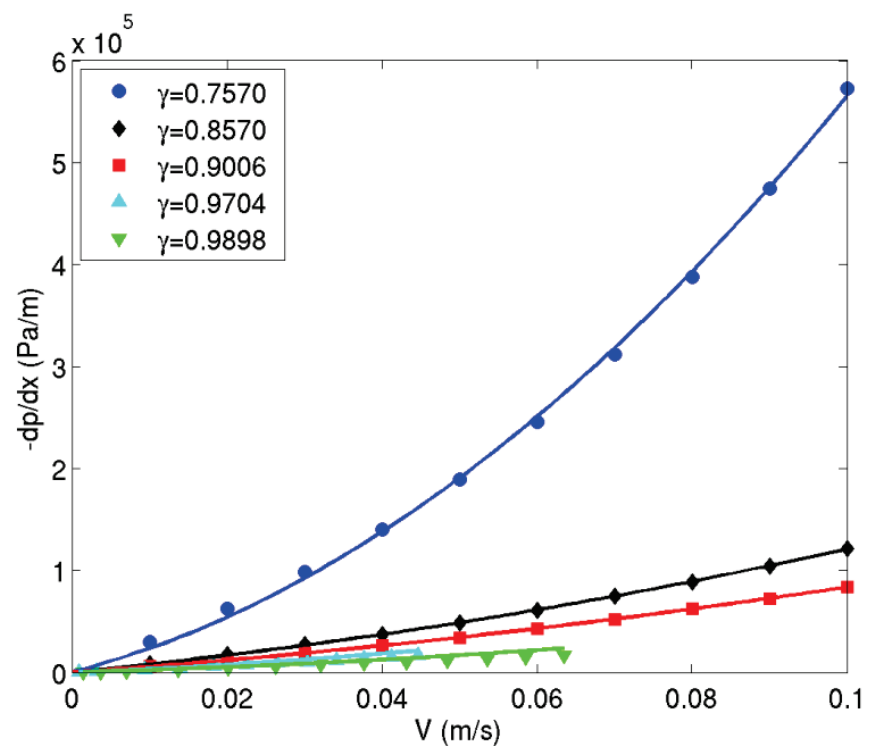

Figure 7-3: Pressure-Drop Rises with Increased Inlet Velocity 
Table 7-1: Permeability and Inertial Coefficient at Different Micro-Foam Porosities

\begin{tabular}{cll}
\hline \hline$\gamma$ & $K\left(\mathrm{~m}^{2}\right)$ & \multicolumn{1}{l}{$F$} \\
\hline 0.7570 & $5.07765 \times 10^{-10}$ & 0.83089 \\
0.8570 & $1.33843 \times 10^{-9}$ & 0.16874 \\
0.9006 & $1.87374 \times 10^{-9}$ & 0.13183 \\
0.9704 & $3.34780 \times 10^{-9}$ & 0.09103 \\
0.9898 & $4.84120 \times 10^{-9}$ & 0.05886 \\
\hline \hline
\end{tabular}

The pressure drop $\left(\frac{d p}{d x}=-\frac{\mu_{f}}{K} V-\frac{\rho_{f} F}{\sqrt{K}} V^{2}\right)$ was used to calculate the permeability $K$ and inertial coefficient $F$ for the foam porosities listed in Table 7-1. It can be seen that as the porosity increases, the permeability rises and the inertial coefficient decreases, which means that viscous and inertial resistance reduce at larger porosity. The inertial coefficient at porosity 0.9898 is very close to the value obtained by Vafai and Tien [213]. For the porosity $(0.757)$ and the permeability used in the current micro-foam, the calculated Darcy-modified Rayleigh number $\left(\frac{g \rho \beta K H \Delta T}{\alpha \mu}\right)^{\frac{1}{2}}$ is far less than 1 , indicating that the heat transfer is conduction-dominant [228]. It has also been observed in recent experiments on metal foams [196] that the buoyancy-driven flow in melted paraffin wax is totally counteracted by resistances once the porosity is lower than 0.884 . Therefore, natural convection can be reasonably neglected in the current study.

\subsubsection{Problem Setup}

The physical model and geometry of the micro-foam used in the DNS and volumeaveraged simulations is shownFigure 7-4. Figure 7-4(a) shows the micro-foam structure which is an array of solid cube cells with nine spherical pores arranged in a BCC lattice. The size of the simulated structure is $0.2 \mathrm{~cm} \times 2.56 \mathrm{~cm} \times 0.2 \mathrm{~cm}$ to keep the computational cost affordable. In the Y-direction, there are 64 unit cells with each unit cell of $400 \mu \mathrm{m}$. The pore size is $360 \mu \mathrm{m}$ in diameter and the foam porosity is 0.757 . The $2.56-\mathrm{cm}$ cube in Figure $7-4$ (b) is a simplified model used for the volume-averaged simulation. In both DNS and volume-averaged simulations, the boundary conditions specified are constant heat flux $\left(q_{\text {top }}^{\prime \prime}=2,4\right.$, or $\left.6 \mathrm{~W} / \mathrm{cm}^{2}\right)$ for the top surface and adiabatic for the bottom surface. In the volume-averaged simulations, the side walls are insulated. In DNS simulations, symmetric boundary conditions are specified on side walls. As will be shown in Section 7.3, the melting front evolves to be one-dimensional from volume-averaged simulations, thus the smaller cross-section of the $\mathrm{X}-\mathrm{Z}$ plane in the model is not expected to affect the comparison between the simulations. Aluminum foam and docosane (paraffin wax with $\mathrm{C}_{22} \mathrm{H}_{46}$ ) PCM were considered and their thermophysical properties are listed in Table 7-2. 

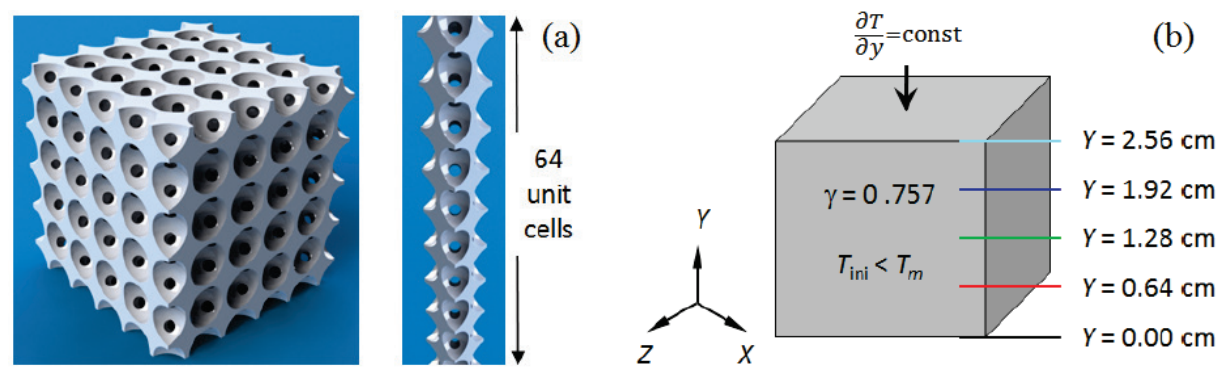

Figure 7-4: Physical Model Used for (a) DNS and (b) Volume-averaged Simulations

Table 7-2: Thermophysical Parameters for PCM and Micro-foam

\begin{tabular}{|c|c|c|c|c|c|}
\hline & $\begin{array}{c}\rho \\
\left(\mathrm{kg} / \mathrm{m}^{3}\right)\end{array}$ & $\begin{array}{l}\quad c_{p} \\
(\mathrm{~kJ} / \mathrm{kg}- \\
\mathrm{K})\end{array}$ & $\begin{array}{l}k \\
(\mathrm{~W} / \mathrm{m}-\mathrm{K})\end{array}$ & $\begin{array}{l}L \\
(\mathrm{~kJ} / \mathrm{kg})\end{array}$ & $\begin{array}{l}T_{m} \\
(\mathrm{~K})\end{array}$ \\
\hline PCM & 785 & 2.89 & 0.4 & 260 & 317 \\
\hline Micro-foam & 2719 & 0.871 & 202.4 & N/A & N/A \\
\hline
\end{tabular}

It was assumed that the micro-foam is fully saturated with docosane and the volume change of PCM on melting was not taken into account. The initial temperature was $300 \mathrm{~K}$, which is below the melting temperature of PCM. From the analysis in Section 7.2.4, we know that the effect of natural convection is not important, thus only heat conduction in the micro-foam was considered.

In the two-temperature model, the PCM and the micro-foam on the top wall have different surface areas, $A_{f}$ and $A_{s}$ respectively. The heat fluxes for the two is proportionate to their surface areas, such that $q_{f, t o p}^{\prime \prime}=\frac{q_{t o p}^{\prime \prime} A_{f}}{A_{f}+A_{s}}$ and $q_{s, t o p}^{\prime \prime}=\frac{q_{t o p}^{\prime \prime} A_{s}}{A_{f}+A_{s}}$. Note that the surface area fraction $\frac{A_{f}}{A_{f}+A_{s}}$ is not equal to the porosity $\gamma$, which is the local volume fraction. For the BCC type ideal foam structure we have $\frac{A_{f}}{A_{f}+A_{s}}=0.636$.

The commercial CFD package ANSYS FLUENT 13.0 was used and simulations were carried using the supercomputing facilities at the AFRL DoD Supercomputer Resource Center (DSRC). To apply the two-temperature model in FLUENT, $T_{s}$ in equation (10) was implemented as a user-defined scalar and unsteady, diffusion, and source terms (heat transfer between PCM and the micro-foam) were implemented as user defined functions. The convergence criterion of absolute scaled residual for energy equation was $10^{-9}$ for volume-averaged simulations and $10^{-11}$ for the DNS. Grid- and timestep independent studies were carried out. For volume-averaged simulations (both one- and two-temperature models), three sets of grids with $30^{3}, 45^{3}$, and $60^{3}$ cells were used, and it was found that the grid with $45^{3}$ cells were sufficient to produce grid-independent result. Also, three timesteps $d t=0.04,0.02$, and $0.01 \mathrm{~s}$ were chosen, and $d t=0.02 \mathrm{~s}$ was found to be sufficient to get timestep independent result. All subsequent simulations were run with $45^{3}$ cells and $d t=0.02 \mathrm{~s}$. The simulations were carried out on a SGI Altix 4700 supercomputer, with 18 nodes and $512 \mathrm{CPU}$ cores per node. Using $32 \mathrm{CPU}$ cores, it takes about 8 hours to complete a typical volume-averaged two-temperature simulation.

For DNS, three sets of grids of $6.3,9.1$, and 12.5 million cells were conducted and it was found that 9.1 million cells were adequate to obtain grid-independent results. Five timesteps $d t=$ $0.01,0.008,0.004,0.002$, and $0.001 \mathrm{~s}$ were tested and $d t=0.008 \mathrm{~s}$ was found to be sufficient to

DISTRIBUTION STATEMENT A: Approved for public release. Distribution is unlimited. 
obtain timestep independent results. All subsequent simulations were run with $9.1 \times 10^{6}$ grid points and $0.008 \mathrm{~s}$ timestep. On the same supercomputer, a typical simulation on $192 \mathrm{CPU}$ cores takes about 172 hours to complete.

\subsection{Results and Discussions}

\subsubsection{One-Temperature Simulation}

Results from one-temperature volume-averaged simulation with Progelhoff effective thermal conductivity are shown in Figure 7-5. Typical PCM melting front were found to be essentially one-dimensional (Figure 7-5a.). Figure 7-5(b) shows the temperature history of five different Y-planes for $q_{\text {top }}^{\prime \prime}=4 \mathrm{~W} / \mathrm{cm}^{2}$. The temperature variation on any Y-plane can be divided into four stages: the pre-melting stage, the melting stage, the waiting stage (in this stage the local melting has completed but not all the PCM in the system has melted), and the post-melting stage. The temperature in the melting stage keeps nearly constant due to phase change. On the top wall $(\mathrm{Y}=2.56 \mathrm{~cm})$, it can be seen that the pre-melting stage is short, and it takes approximately $10 \mathrm{~s}$ for the temperature to reach the melting point. The melting process takes additional 8 seconds to melt all the PCM on the top surface. In the waiting state, which takes about $145 \mathrm{~s}$, the temperature rises mildly, since most of the heat flux is being used to melt the solid PCM underneath. In the post-melting state, due to sensible heat transfer, the temperature rises with a relatively higher rate. The temperature profiles on other Y-planes are similar except that as the planes move away from the top surface, the pre-melting stage gets longer and waiting stage gets shorter. It can be estimated that the melting of PCM for the whole structure was completed approximately within $145 \mathrm{~s}$. As expected, in the post melting stage where the temperature rise is only due to sensible heating, the different planes show the same rate of increase of temperature. A similar four-stage temperature profiles are also observed for $q_{\text {top }}^{\prime \prime}=6 \mathrm{~W} / \mathrm{cm}^{2}$. In the case of $q_{\text {top }}^{\prime \prime}=2 \mathrm{~W} / \mathrm{cm}^{2}$, the $200 \mathrm{~s}$ simulation time was not sufficient to complete PCM melting and a four-stage temperature variation was not observed.
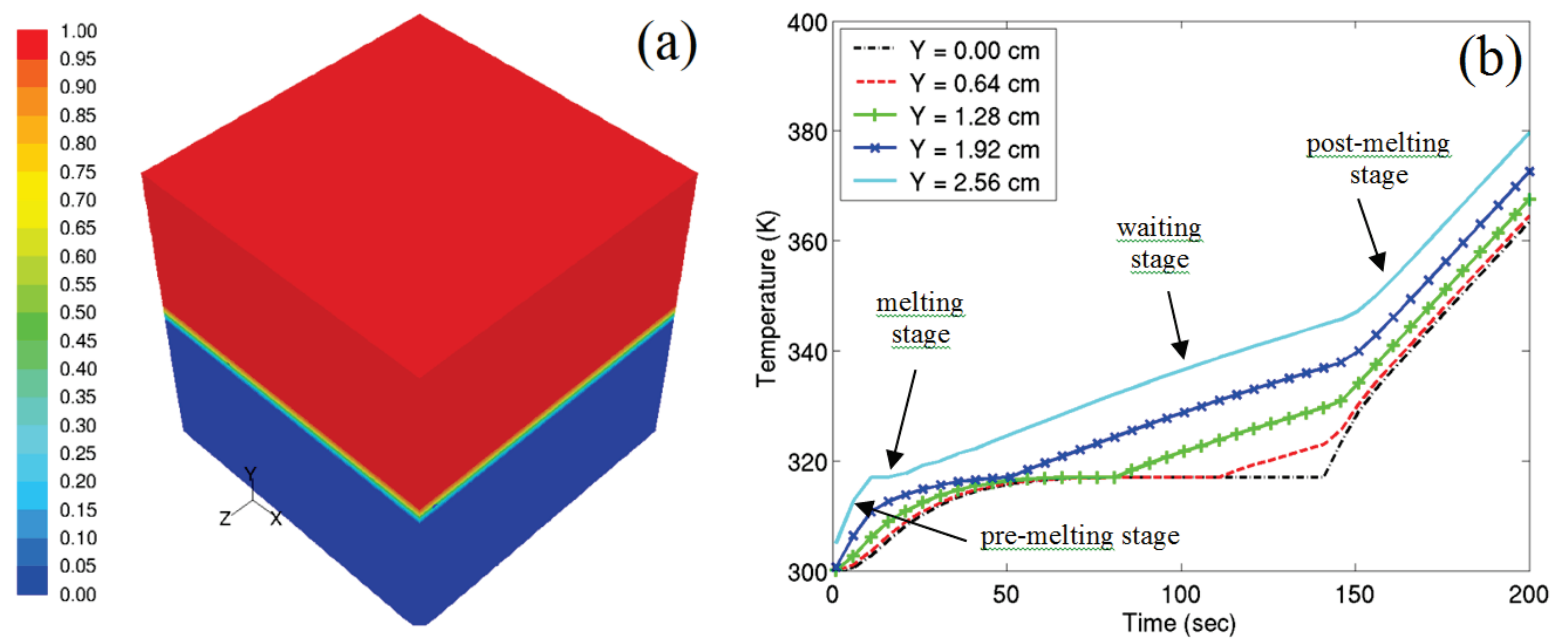

Figure 7-5: One-Temperature Simulation

( $q_{\text {top }}^{\prime \prime}=4 \mathrm{~W} / \mathrm{cm}^{2}$ with the Progelhoff model used for effective thermal conductivity. (a) Onedimensional melting front with half of the PCM melted. 0 and 1 stand for pure solid and pure liquid, respectively; (b) Four-stage temperature profiles.)

DISTRIBUTION STATEMENT A: Approved for public release. Distribution is unlimited. 


\subsubsection{Two-Temperature Simulation}

In the two-temperature models, the micro-foam and the PCM have different temperatures at the interface. The simulated melting front and the temperature profiles are similar to those in Figure 7-5, are hence not presented here. Figure 7-6 shows the difference of the area-average temperature, $\delta \bar{T}=\bar{T}_{s}-\bar{T}_{f}$, in which $\bar{T}_{s}$ and $\bar{T}_{f}$ are respectively the average temperature of the micro-foam and the PCM on specified Y-planes (top-wall heat flux $q_{\text {top }}^{\prime \prime}=4 \mathrm{~W} / \mathrm{cm}^{2}$ ). The thermal conductivities were calculated using both arithmetic mean and the Progelhoff model and their influence on the computed temperature difference is presented in Figure 7-6. On the top wall $(\mathrm{Y}=$ $2.56 \mathrm{~cm})$, the temperature difference $\delta \bar{T}$ is negative. The effective thermal conductivity of PCM $\left(k_{f, e f f}\right)$ is less than the effective thermal conductivity of micro-foam $\left(k_{s, e f f}\right)$. Therefore, the temperature gradient in the PCM region is larger and, correspondingly, $\bar{T}_{s}<\bar{T}_{f}$ for the same heat flux. This $\delta \bar{T}$ decreases rapidly at $t \sim 10 \mathrm{~s}$ when PCM starts to melt, during which the rise in temperature of PCM slows down as most of the absorbed heat goes towards melting, but the microfoam temperature still rises steadily. Once the PCM on the top wall finishes melting (approximately at $t=20 \mathrm{~s}$ ), $\delta \bar{T}$ shows a peak and then levels off until all the PCM in the system is melted. The two-temperature simulation using the Progelhoff model predicts earlier phase change initiation compared to using arithmetic mean. This is consistent with lower effective thermal conductivity calculated by Progelhoff model resulting in higher average temperature.

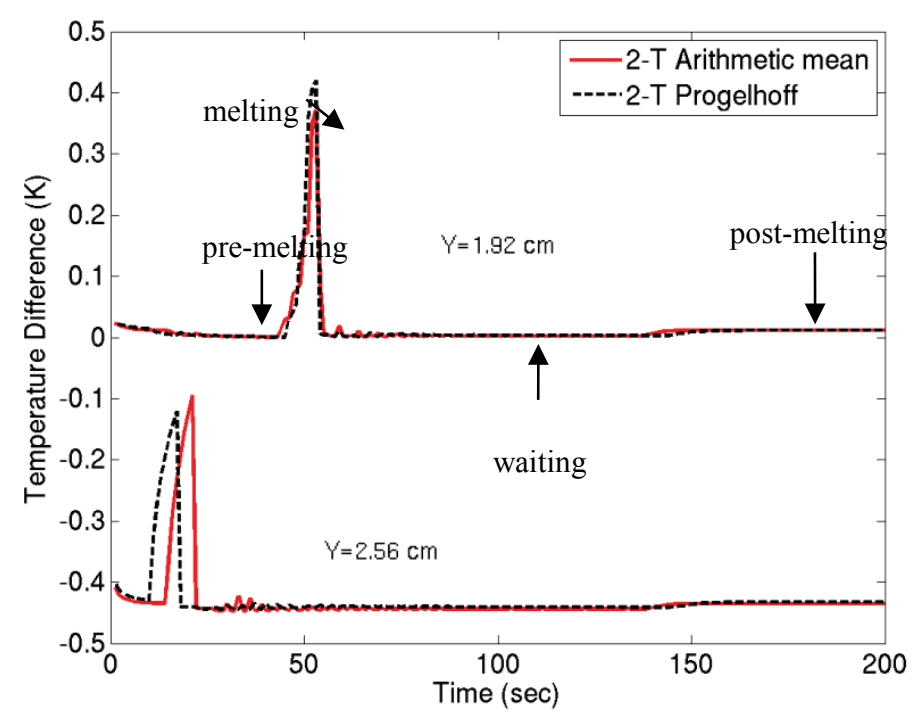

Figure 7-6: Difference in Average Temperatures of Micro-foam and PCM

(Shown: on planes $Y=2.56 \mathrm{~cm}$ and $Y=1.92 \mathrm{~cm}$ for $q_{\text {top }}^{\prime \prime}=4 \mathrm{~W} / \mathrm{cm}^{2}$ using two-temperature simulations.)

Similar time history of $\delta \bar{T}$ is obtained on the plane $\mathrm{Y}=1.92 \mathrm{~cm}$. As expected, the peak in $\delta \bar{T}$ occurs at a delayed time compared to that for the top wall and both Progelhoff model and arithmetic mean exhibit overlapping peaks. Due to the higher thermal conductivity of the microfoam than that of PCM, the foam reaches higher temperature earlier than the PCM, giving rise to a positive $\delta \bar{T}$. 


\subsubsection{Direct Numerical Simulation}

As seen inFigure 7-7, DNS captured the evolution of a three-dimensional melting front and temperature distribution due to the presence of the complex micro-foam structure. InFigure 7-7, we show the liquid PCM volume fraction for a five unit cell system under constant heat flux $q_{\text {top }}^{\prime \prime}=$ $4 \mathrm{~W} / \mathrm{cm}^{2}$. We can see that PCM in the pore of the foam on the top wall starts melting from the center of the pore whereas PCM away from the top wall first melts at the PCM/micro-foam interface and then the melting front spreads to the center of solid PCM balls in the pores. The liquid PCM domain grows with time until all the PCM is melted. It can be clearly seen that even as the PCM is not fully melted in a certain layer, heat is conducted to the micro-foam layer beneath it. The melting front is thus much more complicated than the one-dimensional profile obtained from volume-averaged simulations.
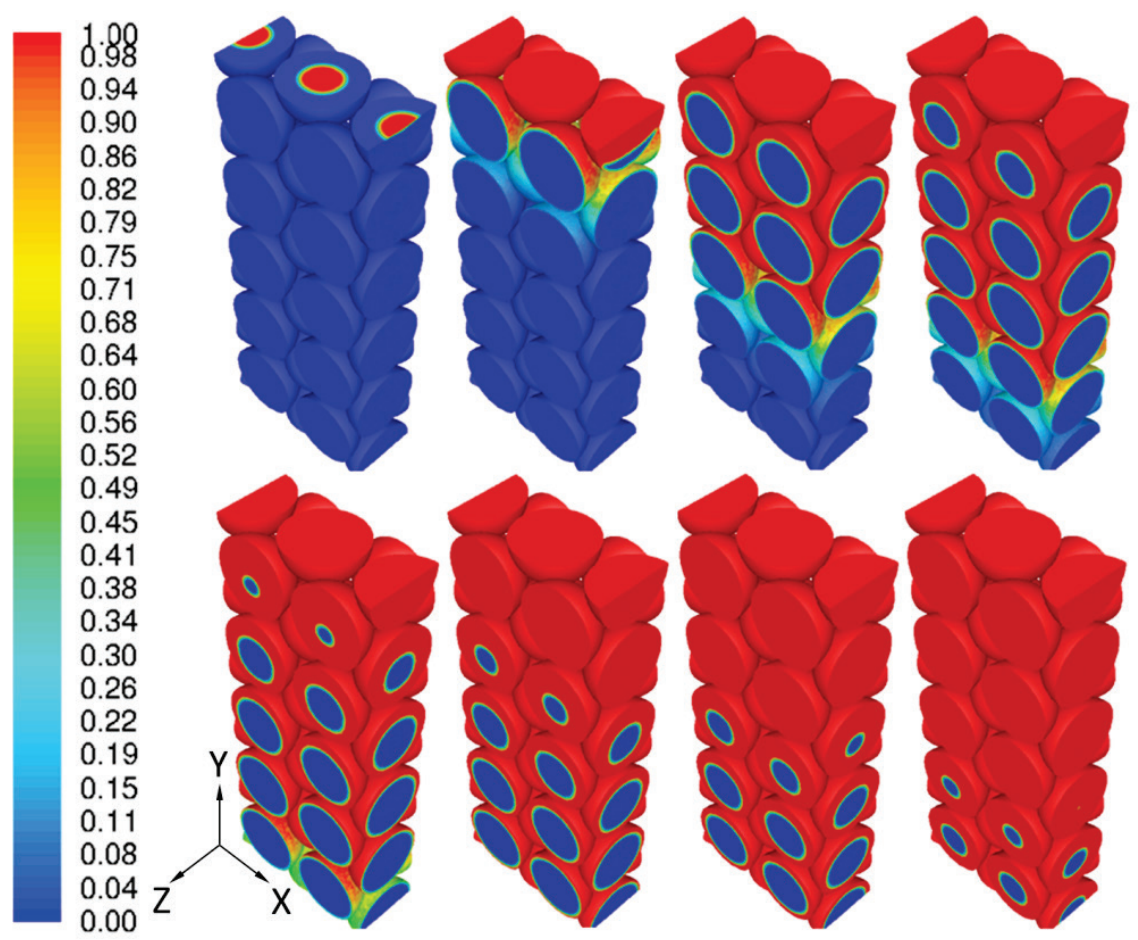

Figure 7-7: Evolution of a 3-D Melting Front and Temperature Distribution

(Left to right, top to bottom: Liquid PCM volume fraction at $t=1.2,2.4,3.6,4.8,6.0,7.2,8.4$, and 9.6 seconds under $q_{\text {top }}^{\prime \prime}=4 \mathrm{~W} / \mathrm{cm}^{2}$ in a five-unit-cell (Y-axis) PCM/micro-foam system. For clarity, the foam is not shown and mirror images of unit cells are shown along the $x$ axis. A contour value of 1 (red) stands for melted PCM and 0 (blue) for solid PCM.)

The temperature distribution in the PCM/foam structure is shown inFigure 7-8. On the top surface, the PCM holds a higher temperature than the micro-foam, since it has a lower thermal conductivity than the foam. However, this temperature difference drops quickly as we move away from the top surface. Similar results were observed for simulations with 64 unit cells. The average temperature difference $\delta \bar{T}$ is shown in Figure $7-9$, in which a peak in $\delta \bar{T}$ is observed during melting. The temperature difference $\delta \bar{T}$ on the top wall $(\mathrm{Y}=2.56 \mathrm{~cm})$ is negative but it is higher in magnitude compared to results obtained from the two-temperature simulations. 


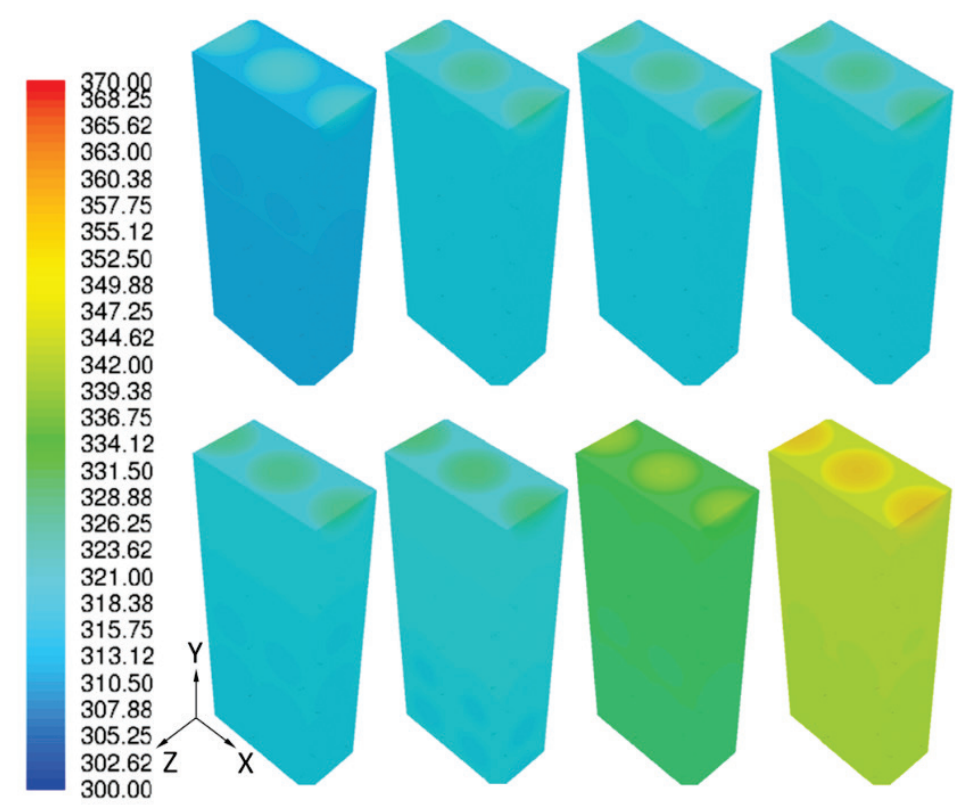

Figure 7-8: Temperature Distribution in the PCM/Foam Structure

(Left to right, top to bottom: Temperature distribution at $t=1.6,3.2,4.8,6.4,8.0,9.6,11.2$, and 12.8 seconds under $q_{\text {top }}^{\prime \prime}=4 \mathrm{~W} / \mathrm{cm}^{2}$ in a five-unit-cell (Y-axis) PCM/micro-foam system.)

This temperature difference gets smaller as we move away from the top surface and is close to zero for $Y=1.92 \mathrm{~cm}$. We also find that for $Y=1.92 \mathrm{~cm}$, the magnitude and position of the peak matches with that from the two-temperature volume averaged simulations, indicating that away from the top surface the two-temperature volume average method is able to capture the gross thermal behavior.

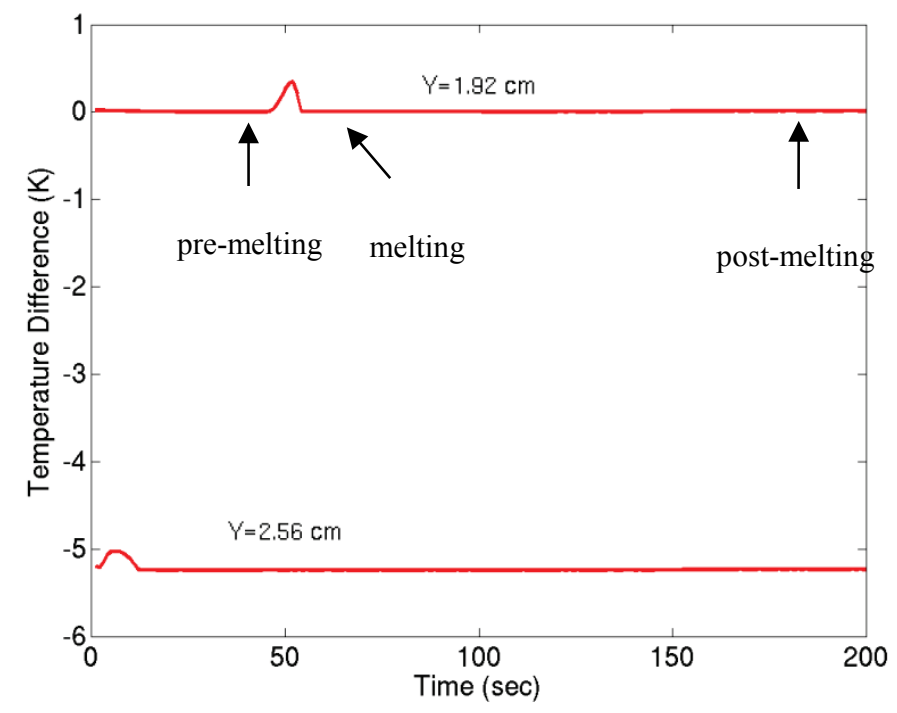

Figure 7-9: Difference in Average Temperature of Micro-Foam and PCM

(Shown: on planes $Y=2.56 \mathrm{~cm}$ and $Y=1.92 \mathrm{~cm}$ for $q_{\text {top }}^{\prime \prime}=4 \mathrm{~W} / \mathrm{cm}^{2}$ using DNS.) 


\subsubsection{Comparison of Temperature Profiles}

Figure 7-10shows the average temperature on the top wall $(\mathrm{Y}=2.56 \mathrm{~cm})$ obtained from the three different methods. As expected, the PCM melts faster when a higher heat flux is applied. For the small heat flux case $\left(q_{\text {top }}^{\prime \prime}=2 \mathrm{~W} / \mathrm{cm}^{2}\right)$ (Figure 7-10a), $200 \mathrm{~s}$ was found to be not long enough to melt the total PCM in the system. As the heat flux $q_{\text {top }}^{\prime \prime}$ increased to $4 \mathrm{~W} / \mathrm{cm}^{2}$ (Figure $7-10 \mathrm{~b}$ ) and $6 \mathrm{~W} / \mathrm{cm}^{2}$ (Figure 7-10c), it takes about $140 \mathrm{~s}$ and $100 \mathrm{~s}$, respectively, to melt the total PCM. It can be seen that both the one-temperature and the two-temperature volume-averaged simulations predict nearly identical results when they use the same effective thermal conductivity model. Comparing the instantaneous temperatures, we can see that DNS predicts the highest temperature, and the volume-averaged simulations with the effective thermal conductivity calculated from arithmetic mean generate the lowest average temperature. 
(a)

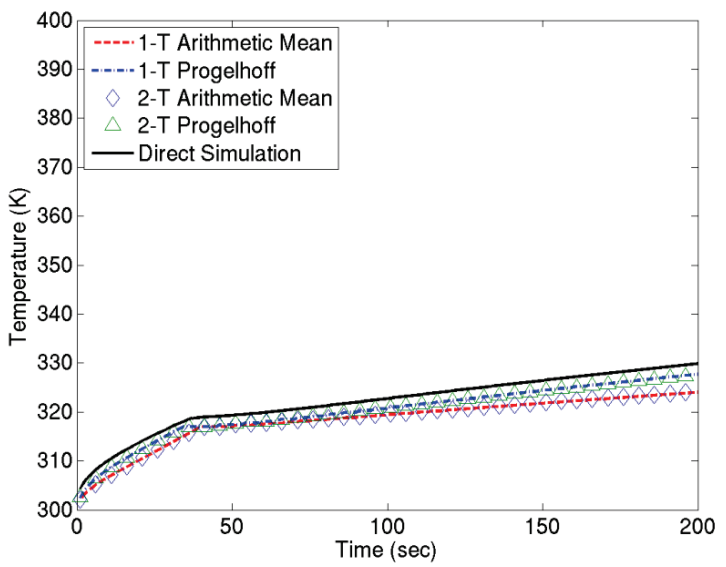

(b)

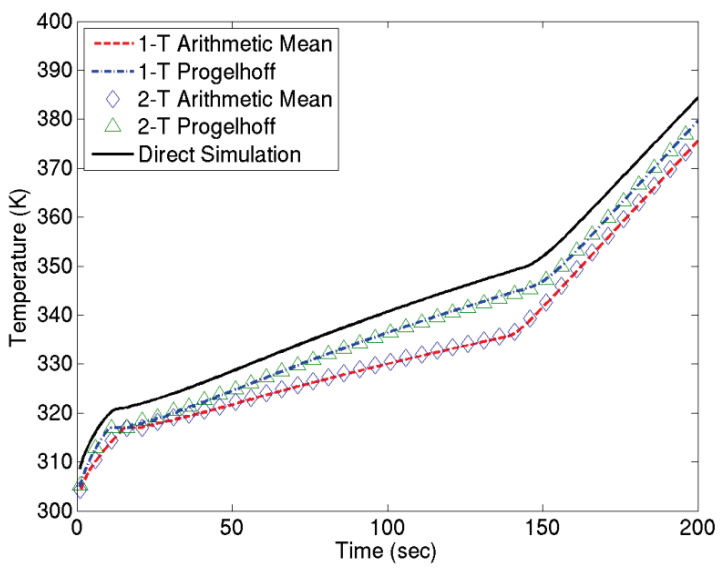

(c)

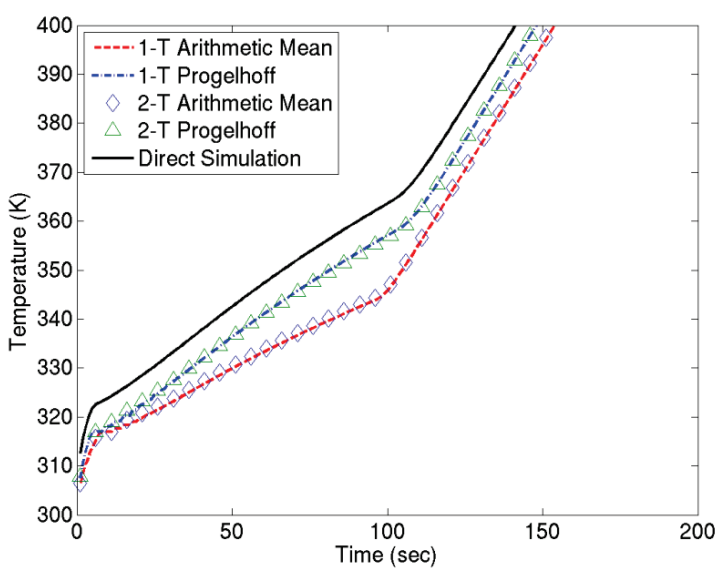

Figure 7-10: Temperature Profile on the Top Wall $(Y=2.56 \mathrm{~cm})$

( (a) $q_{\text {top }}^{\prime \prime}=2 \mathrm{~W} / \mathrm{cm}^{2}$; (b) $q_{\text {top }}^{\prime \prime}=4 \mathrm{~W} / \mathrm{cm}^{2}$, and (c) $q_{\text {top }}^{\prime \prime}=6 \mathrm{~W} / \mathrm{cm}^{2}$.) 
(a)

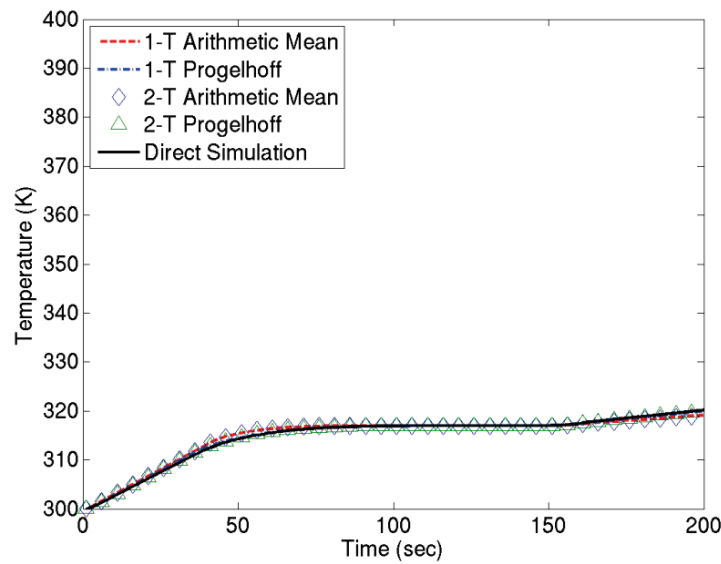

(b)

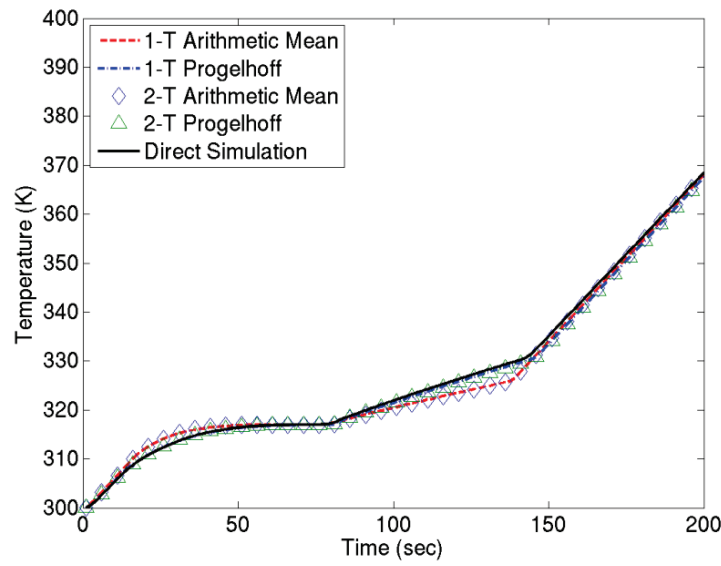

(c)

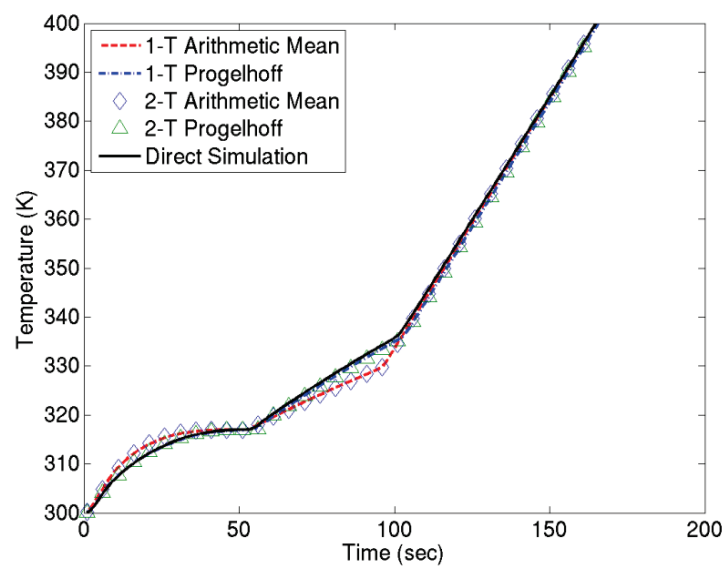

\section{Figure 7-11: Temperature Profile on the Mid-Plane ( $Y=1.28 \mathrm{~cm})$}

$$
\text { ((a) } q_{\text {top }}^{\prime \prime}=2 \mathrm{~W} / \mathrm{cm}^{2} \text {; (b) } q_{\text {top }}^{\prime \prime}=4 \mathrm{~W} / \mathrm{cm}^{2} \text {; (c) } q_{\text {top }}^{\prime \prime}=6 \mathrm{~W} / \mathrm{cm}^{2} \text {.) }
$$

Figure 7-11 shows the temperature profile on the mid-plane $(\mathrm{Y}=1.28 \mathrm{~cm})$. It can be seen that the temperature predictions from three methods are much closer to each other compared to those at the top wall (Figure 7-10). For the low heat flux $\left(q_{t o p}^{\prime \prime}=2 \mathrm{~W} / \mathrm{cm}^{2}\right)$, the temperature profiles obtained from the different methods are almost identical. As the input heat flux rises 
(Figure 7-11b and Figure 7-11c), melting of the PCM in the mid-plane is faster, the waiting stage is shorter, and the slope of the temperature increase in the post-melting stage is steeper.

On the bottom wall $\mathrm{Y}=0 \mathrm{~cm}$ (Figure 7-12), melting stage is directly followed by the post melting stage. The three methods predict similar temperatures except that the volume-averaged simulation with effective thermal conductivity calculated from arithmetic mean, have the highest thermal conductivity and therefore overestimate the transition temperatures.

Figure 7-13(a)-(c) shows the comparison of liquid PCM volume fraction between the DNS and volume-averaged simulations. At the low energy input $\left(q_{\text {top }}^{\prime \prime}=2 \mathrm{~W} / \mathrm{cm}^{2}\right)$ as shown in Figure $7-13$ (a), only $70 \%$ of the total PCM is melted over 200 -second simulations. Higher heat fluxes such as $4 \mathrm{~W} / \mathrm{cm}^{2}$ and $6 \mathrm{~W} / \mathrm{cm}^{2}$ approximately take $140 \mathrm{~s}$ and 100 s to melt the PCM in the whole structure. 
(a)

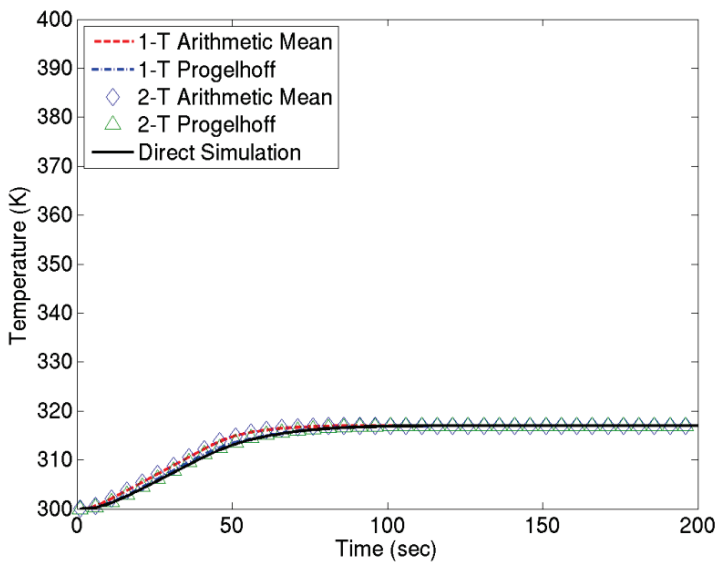

(b)

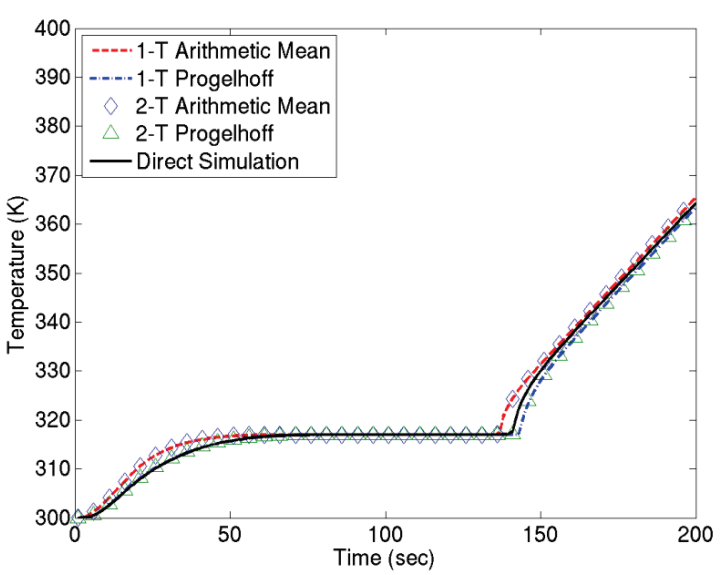

(c)

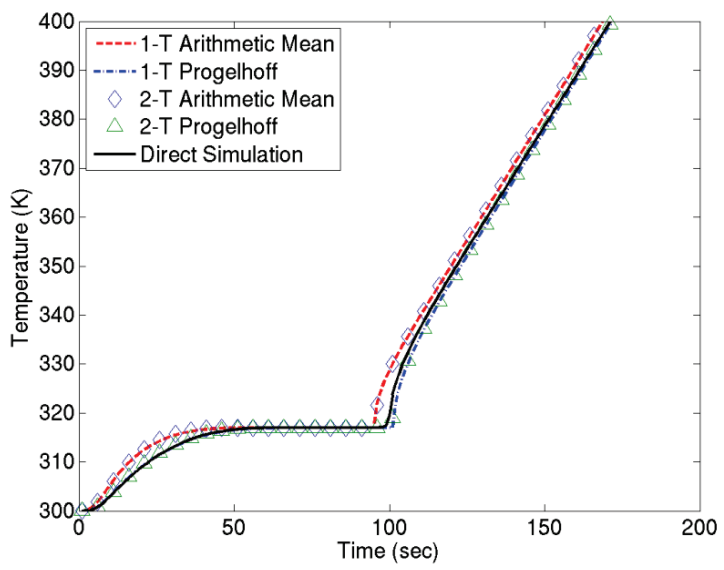

Figure 7-12: Temperature Profile on the Bottom Wall $(\mathrm{Y}=0 \mathrm{~cm})$

((a) $q_{\text {top }}^{\prime \prime}=2 \mathrm{~W} / \mathrm{cm}^{2}$; (b) $q_{\text {top }}^{\prime \prime}=4 \mathrm{~W} / \mathrm{cm}^{2}$; (c) $q_{\text {top }}^{\prime \prime}=6 \mathrm{~W} / \mathrm{cm}^{2}$.) 
(a)

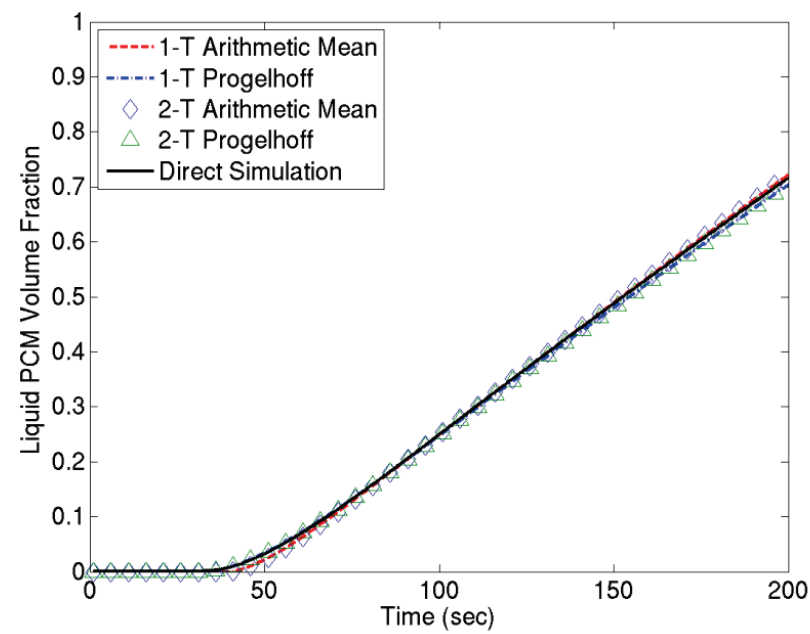

(b)

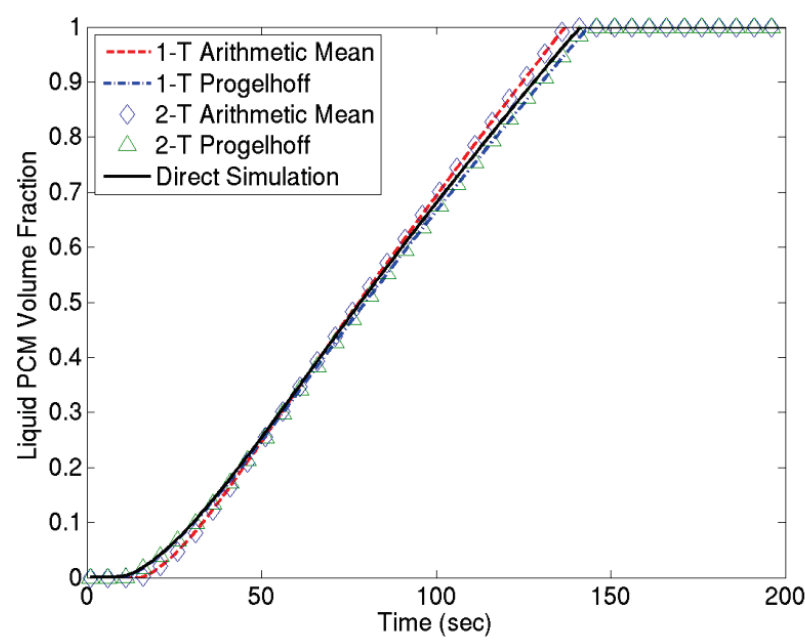

(c)

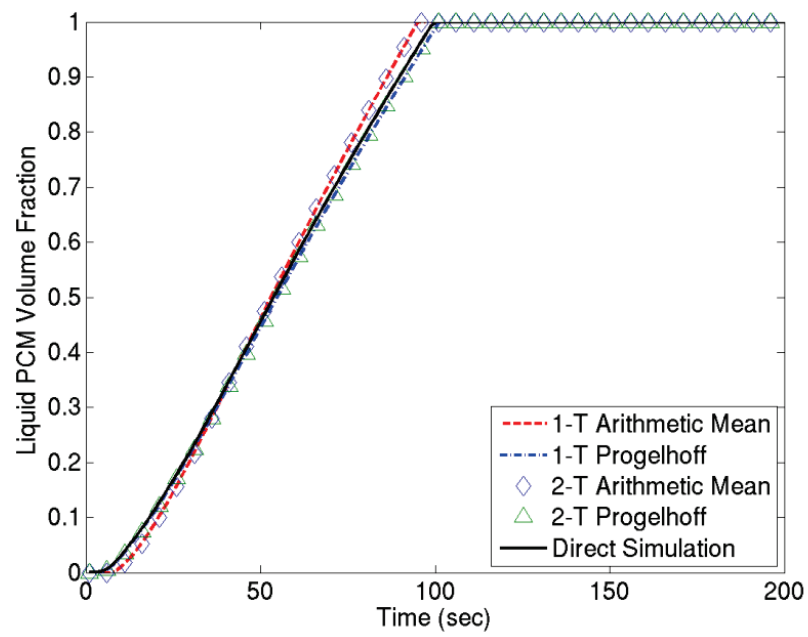

Figure 7-13: Liquid PCM Volume Fraction Profile

(For (a) $q_{\text {top }}^{\prime \prime}=2 \mathrm{~W} / \mathrm{cm}^{2}$; (b) $q_{\text {top }}^{\prime \prime}=4 \mathrm{~W} / \mathrm{cm}^{2}$; and (c) $q_{\text {top }}^{\prime \prime}=6 \mathrm{~W} / \mathrm{cm}^{2}$.) 


\subsection{Conclusions}

Direct numerical simulations provided insight into the complicated heat transfer behavior of PCM/foam systems and a three-dimensional PCM melting front and temperature distribution was observed to evolve under constant heat flux. The pressure drops and interstitial heat transfer coefficients were calculated and it was determined that for a micro-foam porosity of $\gamma=0.757$, the heat transfer is conduction dominated and the effects of natural convection can be neglected. DNS results were used to extract thermo-physical parameters for use in volume averaged methods and the effective thermal conductivity was found to be a non-linear function of the micro-foam porosity and the thermal conductivities of the foam and the PCM. The temperature profiles and PCM melt fraction were compared from results from DNS and one- and twotemperature volume average simulations. Two-temperature volume averaged simulations with effective thermal conductivity based on the nonlinear Progelhoff model with power factor derived from DNS was found to match the DNS results. Both DNS and two-temperature volume-averaged simulations show a four-stage temperature profile during heating: pre-melting, melting, waiting, and post-melting. Considering the much lower computational cost, the two-temperature volumeaveraged method with suitable effective thermal conductivity can be a viable method to provide reasonable prediction of the heat transfer behavior in the PCM/micro-foam system and PCM phase change. 


\section{NATURAL CONVECTION IN A VERTICAL SQUARE ENCLOSURE WITH INSERTED CYLINDERS}

\subsection{Introduction}

Typical natural convection in a rectangular or square enclosure with two differentially heated walls can be roughly classified as horizontal enclosure problem or vertical enclosure problem, depending on whether the applied temperature gradient is perpendicular or parallel to the gravity [229]. The flow motion in enclosures is driven by the buoyancy force and counteracted by the viscosity and thermal diffusion of fluids. Moreover, the flow and heat transfer process are also affected by the aspect ratio and boundary conditions of the enclosure. Therefore, many studies of natural convection in a cavity focus on the effects of relative strength of buoyancy and viscous forces [230], various fluids properties [231-233], aspect ratio [230, 234, 235], and boundary conditions [236-238].

Some studies have also been done on the natural convection in an enclosure with inserted solid bodies [239-245]. For example, the effect of a centered, square, heat-conducting body on natural convection in a vertical square enclosure was examined by House et al. [239]. Depending on the ratio of thermal conductivity between the body and the surrounding fluid, the heat transfer across the enclosure may be enhanced (or reduced) by the body with a thermal conductivity ratio less (or greater) than unity, in comparison to that in the absence of a body. In other words, high thermal conductivity body can reduce the heat transfer across the enclosure. Cesini et al. [244] studied the natural convection of a hot cylinder in air-filled enclosure with cold side walls. The bottom and top walls of the enclosure are set to adiabatic and conductive respectively. They used cavities with height-to-width ratios from 0.5 to 0.9 , and found that non-stationary and oscillating solution were encountered when the Rayleigh number $(R a)$ is above $10^{5}$. Kim et al. [242] investigated the similar problem, but with isothermal cold walls. They placed the cylinder at various locations along the vertical centerline, and found that in the Rayleigh number $(R a)$ range of $10^{3}-10^{6}$, the flow and thermal fields eventually reach steady states with the symmetric shape about the vertical centerline. They also pointed out the effect of the inner cylinder position on fluid flow and heat transfer is important at high Rayleigh number $\left(R a>10^{5}\right)$. Using an adiabatic square cylinder (with an area of 1/9 of the enclosure), Lee et al. [246] studied the Prandtl number (Pr) dependency of the natural convection in a vertical enclosure. The Prandtl number was varied from 0.01 to 7 , and they found that the heat transfer rate increases with the Prandtl number in the range they studied.

There also have some studies been done in the aspect of the size of the cylinder placed in the enclosure. Angeli et al. [241] investigated buoyancy-induced flow regime for the case of a hot cylinder co-axially centered into an air-filled square cavity with isothermal cold walls. They considered the cylinder size effect by varying its non-dimensional diameter from 0.2 to 0.8 , and derived a correlating equation of the average Nusselt number on the cylinder as a function of both the Rayleigh number and the diameter-to-side ratio, in the steady-state regime. Bhave et al. [247] put an adiabatic square block in an horizontal enclosure heated and cooled by vertical walls. It was found that for each Rayleigh number and Prandtl number, there exists an optimum block size, below which the wall heat transfer increases with the block size, and above which the wall heat transfer decreases. They also reported that a steady-state heat transfer enhancement of $10 \%$ was obtained for some Rayleigh and Prandtl numbers. Yoon et al. [245] vertically placed two identical hot cylinders of different sizes in a square cavity with cold walls, and studied the natural convection in the regime of steady flow. They found that the heat transfer on horizontal walls is monotonically enhanced as the diameter of the cylinders increases.

DISTRIBUTION STATEMENT A: Approved for public release. Distribution is unlimited. 
Most previous literature studies of the process of natural convection in an eclsousre with inserted block(s) focus on the steady flow regime. Also, the inserted bodies are usally big in the size or the diameter, and can even account for $10 \%$ of the cross-section area in some studies. Such a big inserted body sometimes is hard to be implimented in an engineeing application. In the present study, we mainly investigate the unsteady flow and heat transfer problem in an vertical enclusure with and without the existence of small cylinder(s). The diameter of each cylinder is one-tenth of the characteristic size of the enclousre, therefore the cross-section area of each cylidner takes only $0.79 \%$ area of the enclosure. The Rayleigh number in our study is varied from from $10^{3}$ (covnection regime) to $10^{6}$ (conduction regime). The fluid media we used are air, water, liquid gallium, therefore, cover the Prandtl number around unity, greater, and smaller than unity respectively.

\subsection{Mathematical Formulation}

With incompressible flow and Boussinesq approximation applied, natural convection problems can be summarized as the following control equations,

$$
\begin{gathered}
\nabla \cdot \mathbf{u}^{*}=0 \\
\frac{\partial \mathbf{u}^{*}}{\partial t^{*}}+\mathbf{u}^{*} \cdot \nabla \mathbf{u}^{*}=-\frac{1}{\rho_{0}} \nabla p^{*}+v \nabla^{2} \mathbf{u}^{*}+\mathbf{g} \alpha\left(\Delta T^{*}\right) \\
\frac{\partial T^{*}}{\partial t^{*}}+\mathbf{u}^{*} \cdot \nabla T^{*}=\kappa \nabla^{2} T^{*}
\end{gathered}
$$

in which the primitive variables $\mathbf{u}^{*}, p^{*}$ are dimensional velocity and pressure respectively. $T^{*}$ is the dimensional temperature. The thermal expansion coefficient, thermal diffusivity, kinematic viscosity, and the density of the fluid are denoted by $\alpha, \kappa, v$, and $\rho_{0}$ respectively. Taking the length of the square cavity $L$ as the characteristic length, the characteristic velocity and the characteristic time can be given as $\kappa / L$ and $L^{2} / \kappa$. The non-dimensional Navier-Stokes equations and thermal equation then have the form of:

$$
\begin{gathered}
\nabla \cdot \mathbf{u}=0 \\
\frac{\partial \mathbf{u}}{\partial t}+\mathbf{u} \cdot \nabla \mathbf{u}=-\nabla p+\operatorname{Pr} \nabla^{2} \mathbf{u}+\operatorname{RaPr} T \\
\frac{\partial T}{\partial t}+\mathbf{u} \cdot \nabla T=\nabla^{2} T
\end{gathered}
$$

in which the non-dimensional Rayleigh number, $R a\left(=g \alpha \Delta T L^{3} / \nu \kappa\right)$, is the ratio of the buoyancy force to the viscous force. The Prandtl number $\operatorname{Pr}(=v / \kappa)$, the ratio of the kinematic to the thermal diffusivity, is a property depending on fluids only. The flow and heat transfer process in the enclosure are highly dependent on $R a, P r$, the aspect ratio (AR), and the boundary conditions [235]. The velocity boundary condition at all walls in our study are non-slip and impermeable, i.e., $\mathbf{u}=0$. The temperatures at the bottom and top walls are kept as unity and zero respectively. Adiabatic boundary conditions are applied on side walls. The temperature on the inside cylinder is set to be zero. The local Nusselt number is defined as $N u=-\frac{L}{\Delta T} \frac{\partial T}{\partial n}$, indicating the ratio of convective to conductive heat transfer across the surface. The surface-averaged Nusselt number, $N u_{A}$, is then calculated by integrating the local Nusselt number along the surface. For convection 
dominated heat transfer, the Nusselt number is far greater than unity. The coordinate origin is placed at the left, low corner of the enclosure.

\subsection{Numerical Method and Validation}

\subsubsection{Numerical Method}

The Navier-Stokes equations are discretized using a cell-centered, collocated arrangement of the primitive variables $(\mathbf{u}, p)$ and integrated in time using fractional step method [16]. Secondorder central difference schemes in space are employed to both convection and diffusion terms in momentum and temperature equations. More details on numerical algorithm and immersed boundary treatment, as well as validations on the CFD solver can also be found in [28].

\subsubsection{Initial Temperature Dependence}

We first studied the initial temperature dependence using a cavity horizontal temperature difference and adiabatic top and bottom walls. The Prandtl number and Rayleigh number are 0.71 and $10^{3}$ respectively. The temperature fields were initialized as uniform zero and linear distribution (in x-direction), respectively. Figure 8-1shows the time course of the velocity and temperature at the cavity center (a), and the surface-averaged Nusselt number $N u_{A}$ at the cold wall $(x=1)$.

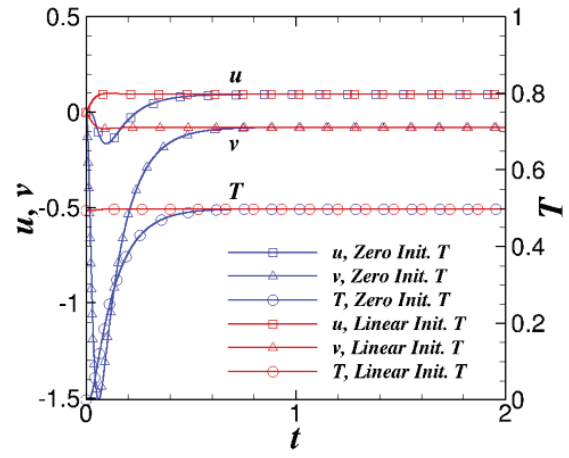

(a) Velocity and temperature history at the cavity center

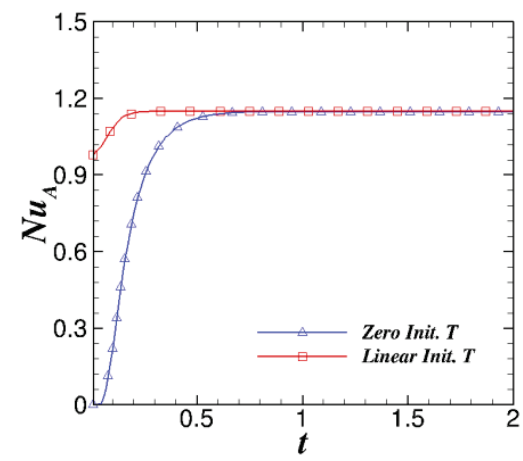

(b) Surface-averaged Nusselt number at the cold wall

Figure 8-1: Temperature Field Dependence on Initial Conditions

$$
\left(\operatorname{Pr}=0.71, R a=10^{3}\right. \text {.) }
$$

Since the Rayleigh number is below the critical number, the heat transfer in the cavity is dominated by conduction. The temperature at the cavity center is therefore around 0.5 . It can be seen that the two initial temperature distributions mainly affect the results in the transient stage. The linear temperature distribution approaches steady state around three times faster than the zero initial temperature distribution. Results from the two initial conditions converge to almost the same value after $t>0.8$. The $N u_{A}$ values at steady state have a difference of $0.16 \%$ between the two initial temperature distributions, for this particular case. We thus use the linear initial temperature distribution in all the following simulations.

\subsubsection{Validation}

\subsubsection{Square cavity with horizontal temperature gradient $(P r=0.71)$}

Table 8-1 lists the surface-averaged Nusselt number at the hot wall, and the comparison with previous literature results is reasonably good. 
Table 8-1: Comparison on Nusselt Number for a Square Cavity with Lateral Temperature Difference

\begin{tabular}{lllll}
\hline$R a$ & $10^{3}$ & $10^{4}$ & $10^{5}$ & $10^{6}$ \\
\hline Ref. [239] & 1.118 & 2.254 & 4.561 & 8.923 \\
Ref. [247] & 1.111 & 2.24 & 4.502 & 8.8 \\
Ref. [248] & 1.117 & 2.238 & 4.509 & 8.717 \\
Ref. [249] & 1.127 & 2.247 & 4.523 & 8.805 \\
Present & 1.135 & 2.256 & 4.531 & 8.815 \\
\hline
\end{tabular}

Simulation of a square cavity with horizontal temperature gradient was conducted as a validation. The Prandtl number of the fluid was 0.71 , and the Rayleigh number was varied from $10^{3}$ to $10^{6}$. Figure $8-2$ shows the temperature field and the streamlines in the cavity at the selected Rayleigh numbers. At the lower left and upper right ends of the cavity, the isotherms are almost parallel to the side wall, indicating a substantial temperature gradient near these regions. In other words, the flow is driven by the strong buoyancy force in these two corners; while the flow in the interior region of the cavity is a consequence of the continuity requirement $[250,251]$.
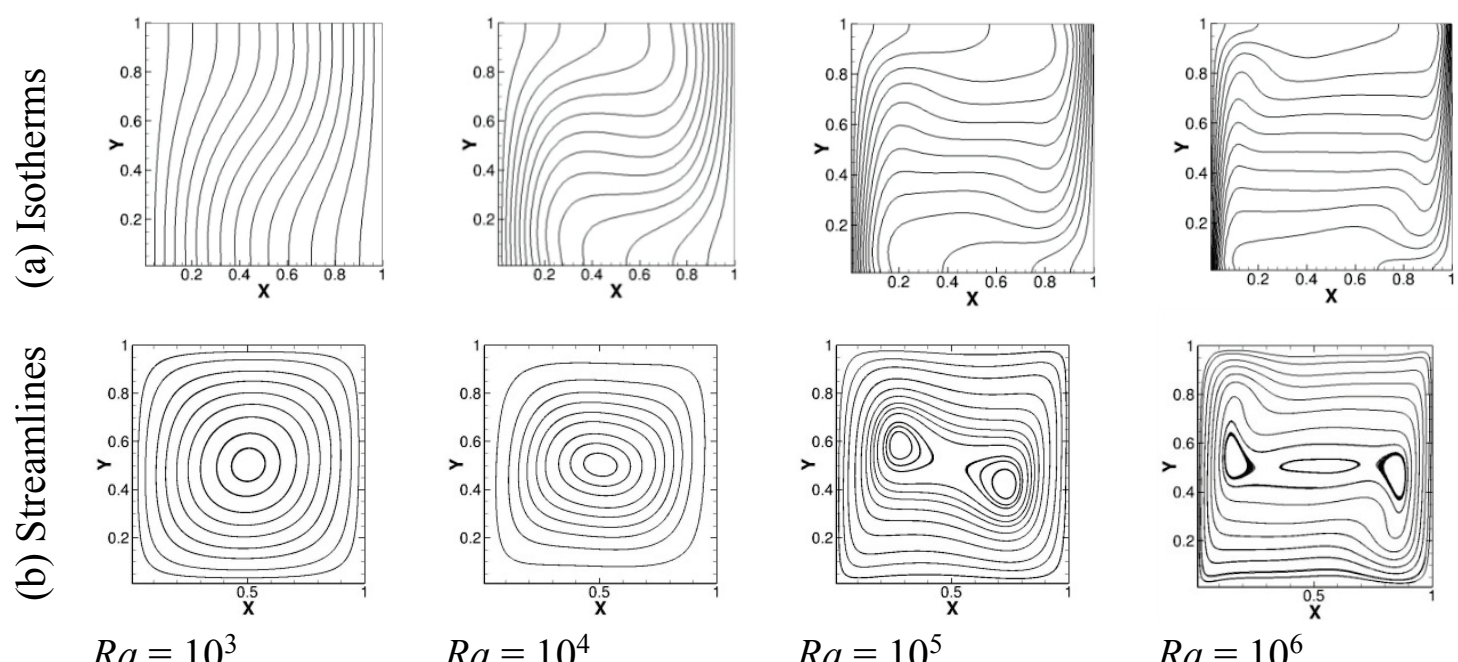

$R a=10^{4}$

$R a=10^{5}$

$R a=10^{6}$

Figure 8-2: Validation I

(Natural convection in a square cavity with horizontal temperature gradient. $\operatorname{Pr}=0.71, \operatorname{Ra}=10^{3}-10^{6}$. (a) Isotherms (b) Streamlines.)

\subsubsection{Isothermal Square Cavity with a Hot Cylinder Inside $(P r=0.71)$}

The second validation shown here was conducted against Kim et al. [242], in which a hot cylinder was placed in a square enclosure with isothermal cold walls. The diameter of the cylinder is one-third of the side of the enclosure. In this case, only steady solution was obtained. The streamlines and isotherms at $R a=10^{3}$ and $R a=10^{6}$, as shown in Figure 8-3are very similar to those from Kim et al. [242]. The Nusselt number along the wall surface also presents a good match.

DISTRIBUTION STATEMENT A: Approved for public release. Distribution is unlimited. 

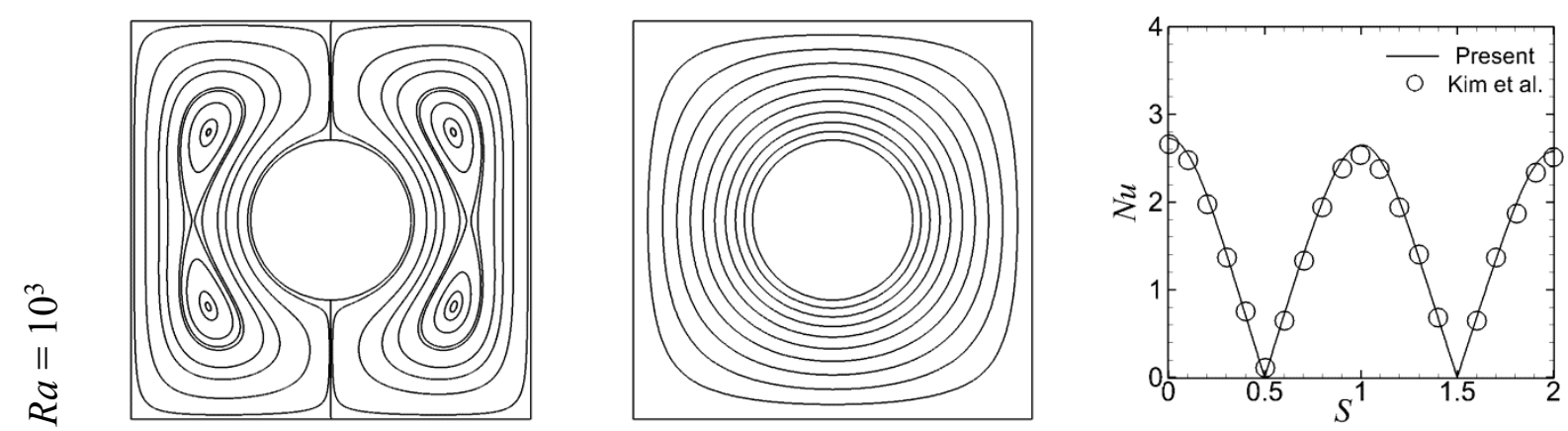

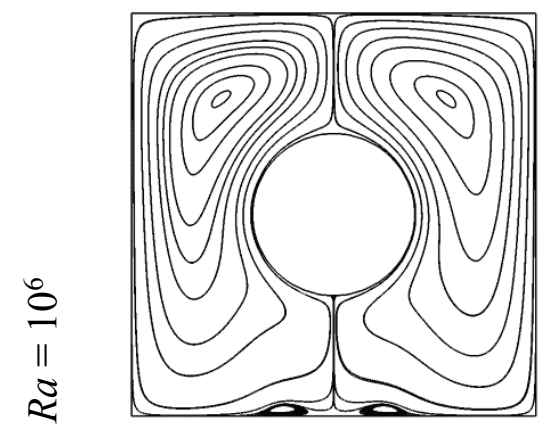

Streamlines

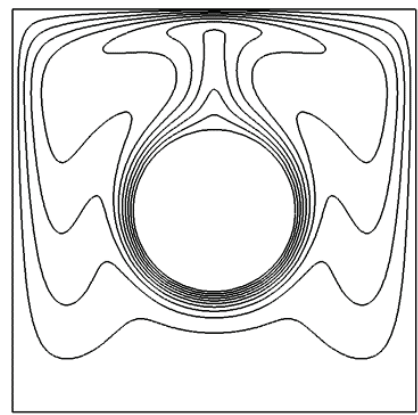

Isotherms

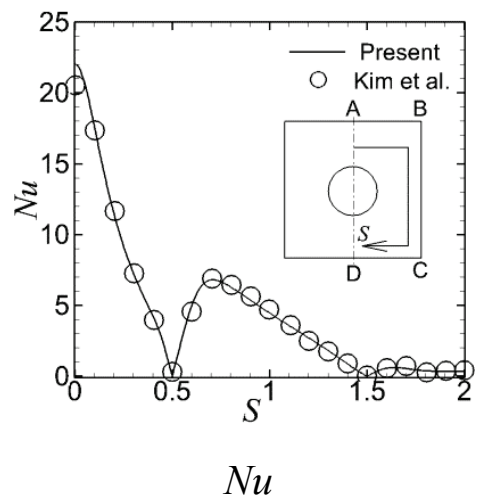

Figure 8-3: Validation II

(A hot cylinder in an enclosure with cold walls. $\mathrm{Pr}=0.71$. The diameter of the cylinder is one-third of the length of the square enclosure.)

\subsubsection{Flow of Low Prandtl Number Fluid in a Horizontal Cavity of Aspect Ratio 2}

The third validation we show here was about the flow in an enclosure with low Prandtl number [252], in which $P r=0.00715$ (corresponding to liquid germanium) and $R a=2300$. The aspect ratio of the enclosure is 2 . High (black) and low (white) temperatures are fixed at the right and the left sides respectively, and the bottom and top surfaces are adiabatic. The obtained temperature profile, streamlines, and vorticity field are shown inFigure 8-4, which are similar to the results in [252]. The solid and dash lines represent positive and negative vorticity respectively. As can be seen, a primary counterclockwise vortex cell tilted in diagonal direction is circulating in the enclosure. Negative vorticity is generated from the four solid boundaries, especially at the bottom and top surfaces, due to the strong shear flow nearby. 


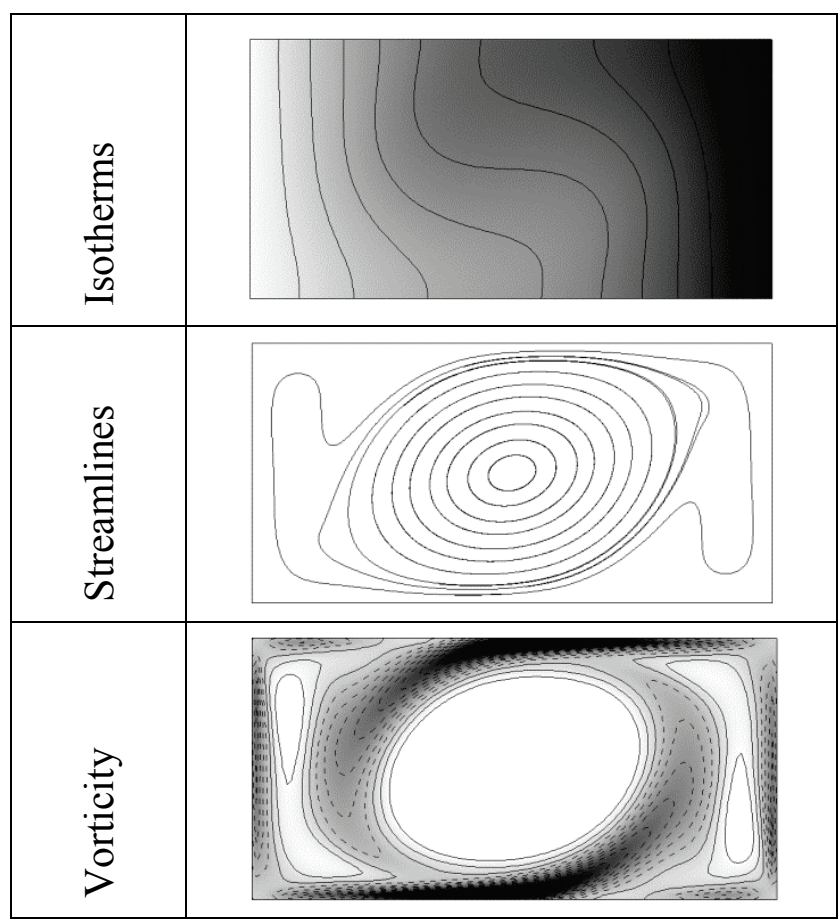

Figure 8-4: Validation III

(Horizontal cavity with $\operatorname{Pr}=0.00715, R a=2300, A R=2$.)

\subsection{Results and Discussion}

In this work, we inserted cold cylinders in a square enclosure, and studied the induced flow motion and heat transfer effect. The diameter of the cylinders in the current study is one-tenth of the enclosure side length. Thus, the area of each cylinder takes only about $0.79 \%$ the enclosure area. When three cylinders are inserted, the area adds up to $2.4 \%$ of the cavity area. If five cylinders are placed in the cavity, the cylinder area is less than $4 \%$. However, we will see that the changes of heat transfer are influential. The cylinders are symmetrically and uniformly placed on the horizontal centerline $(y=L / 2)$ of the enclosure. For example, the cylinder is placed at $(L / 2, L / 2)$ for the one-cylinder case. If two cylinders are inserted, they are placed at $(L / 3, L / 2)$ and $(2 L / 3, L / 2)$ respectively. For three-cylinder case, they are at $(L / 4, L / 2),(L / 2, L / 2)$, and $(3 L / 4, L / 2)$. Positions of more cylinders can be deduced similarly. We started with the two most popular fluid medium, the air $(\operatorname{Pr}=0.71)$ and the water $(\operatorname{Pr}=7.1)$; we then used $\operatorname{Pr}=0.021$ (liquid gallium) as an example of small Prandtl number fluids. In all computational cases, the empty enclosure without any inserted cylinders is taken as the references.

\subsubsection{Pr Number 0.71 (Air)}

\subsubsection{1 $P r=0.71, R a=10^{3}$}

At the low Rayleigh number $10^{3}$, the heat transfer is dominated by the conduction (Figure 8-5). In the empty enclosure without any cylinder inserted, the isotherms are parallel to the bottom and the top surfaces of the enclosure. The steady state surface-averaged Nusselt number $N u_{A}$ at the bottom and top surfaces are close to 1 and are identical, since the energy cross these two surfaces is conserved when the side walls are adiabatic. As one cold cylinder is placed at the enclosure center, the isotherms are bended near the cylinder, and the heat conduction is enhanced in the region from the cylinder to the bottom surface. When the number of cylinders is increased 
to three, $N u_{A}$ is about 2, due to the increase of the temperature gradient between the bottom wall and the center line of the enclosure. The heat conduction process basically occurs in the lower half enclosure, and the top half enclosure is almost shielded by the three cylinders.
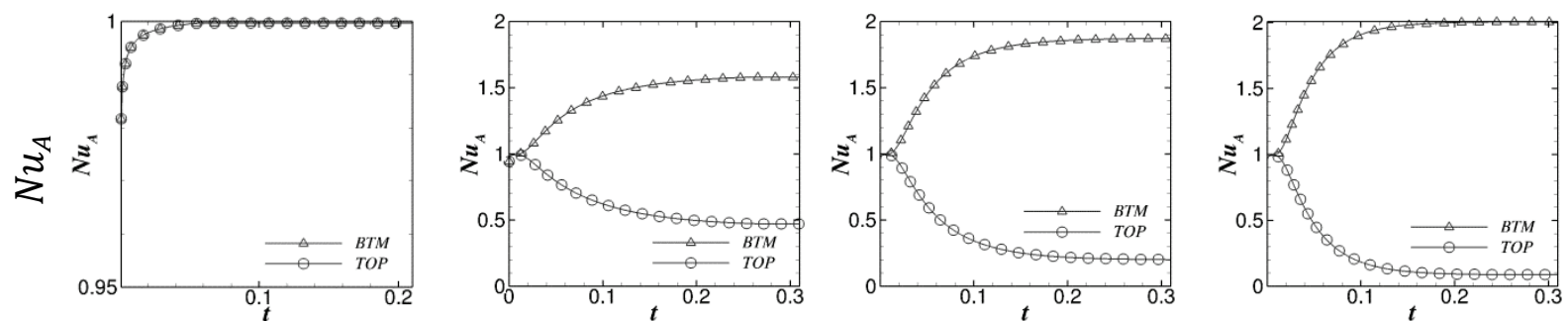

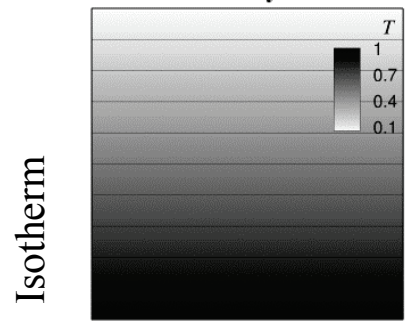

Ref.

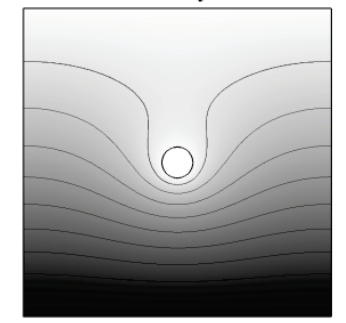

1 Cyl.

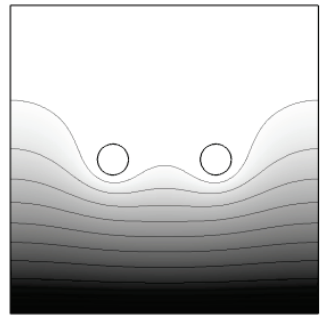

2 Cyls.

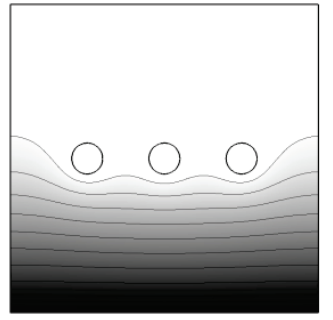

3 Cyls.

Figure 8-5: Surface-averaged Nusselt Number $N u_{A}$ and Isotherms for $\operatorname{Pr}=0.71$ and $R a=$ $10^{3}$

\subsubsection{2 $P r=0.71, R a=10^{4}$}

At $R a=10^{4}$, the heat convection sets in. In all cases, the flow in the empty enclosure is characterized by the bicellular non-oscillatory motion (Figure 8-6). The flow and temperature fields are symmetric about the vertical centerline $(x=0.5)$ of the enclosure. As one or two cylinders are inserted, the heat transfer on the bottom surface is enhanced, with two cylinders give more improvement. When three cylinders are placed, the flow in the enclosure is actually suppressed compared to the two-cylinder case, and the surface-averaged Nusselt number is reduced due to the diminished heat convection. In the one- and three-cylinder cases, the flow around the center cylinder is similar to the creeping flow (no separation). 

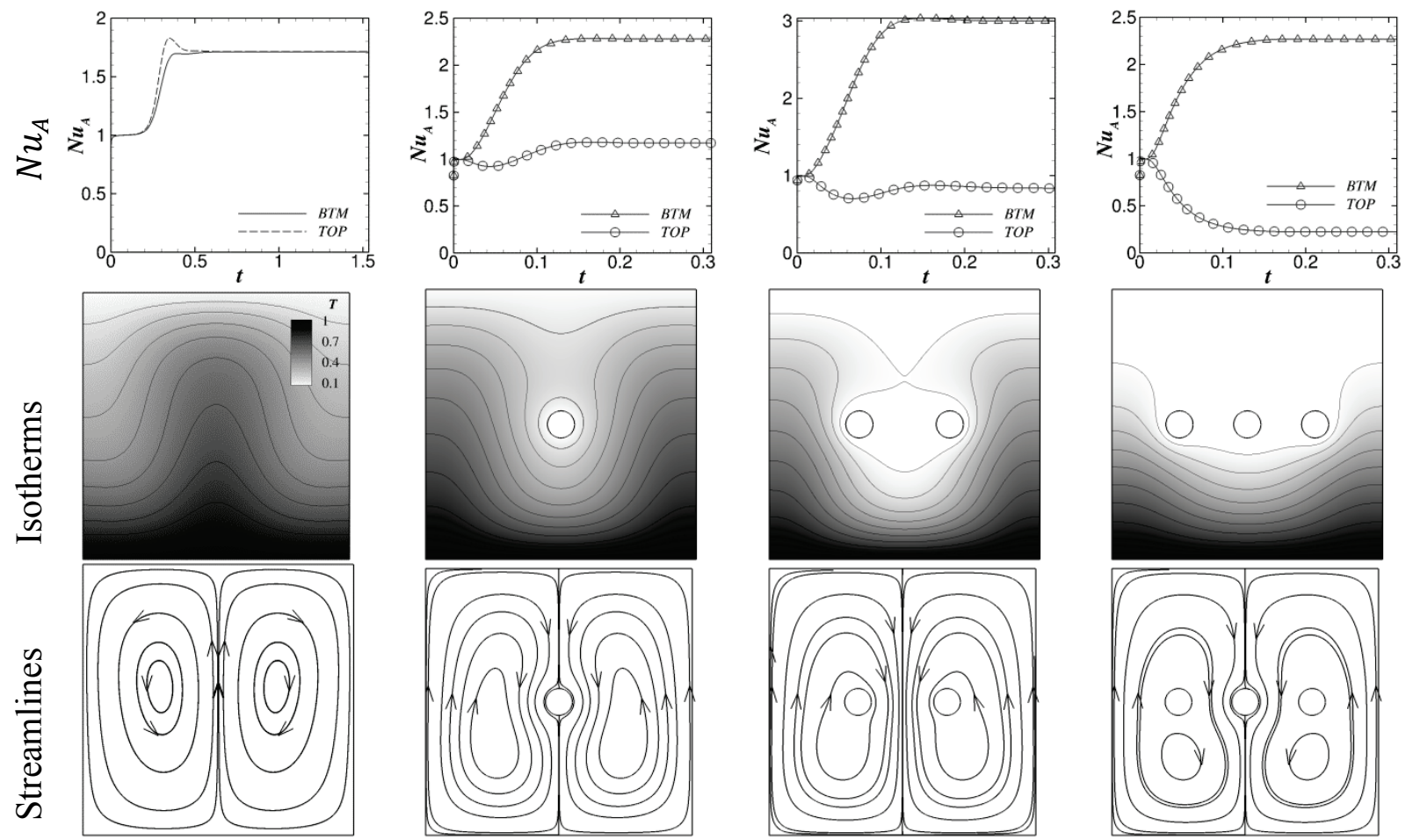

(a) Ref.

(b) 1 Cyl.

(c) 2 Cyls.

(d) 3 Cyls.

Figure 8-6 $\mathrm{Nu}_{A}$, Isotherms and Streamlines for $\mathrm{Pr}=0.71$ and $R a=10^{4}$

\subsubsection{3 $\operatorname{Pr}=0.71, \operatorname{Ra}=10^{5}$}

As the Rayleigh number increased to $10^{5}$, the flow symmetry is broken and the flow structure in the empty enclosure is dominated by a single cell (Figure 8-7). Two small corner vortices are aligned diagonally. Similar streamlines and isotherms were also reported in Huelsz et al. [253]. The flow structure is antisymmetric about the center of the enclosure. Note both the single cell at $R a=10^{5}$ and the bicellular structure at $R a=10^{4}$ shown in Figure 8-6 are stable [229]. Therefore, once the steady flow is developed, the obtained Nusselt number at the hot surface is a constant around 4.1, which is similar to the result of Corcione [236].

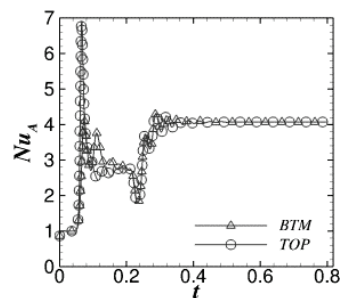

(a) $N u_{A}$

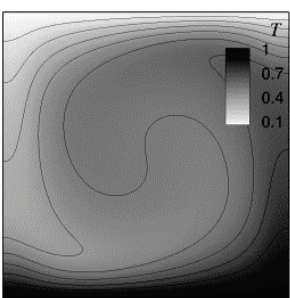

(b) Isotherms

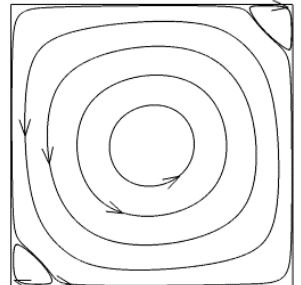

(c) Streamlines

Figure 8-7: Reference Case of $P r=0.71$ and $R a=10^{5}$

Figure 8-8 shows the surface-averaged Nusselt number for the case of $P r=0.71$ and $R a=$ $10^{5}$, with one cylinder in the enclosure. The flow experiences a short quasi-steady status (Figure $8-8$ b), which is unstable and eventually an oscillation solution is developed (Figure 8-8c). The isotherms and flow structures are shown in Figure 8-9. At the very beginning (Figure 8-9a), bicellular flow is formed, similar to the structure at $R a=10^{4}$. Each vortex expands to the whole depth of the enclosure, and the flow and thermal plume rise along the two adiabatic side walls. As

DISTRIBUTION STATEMENT A: Approved for public release. Distribution is unlimited. 
the thermal induced buoyancy drops at the upper part of the enclosure, two symmetric corner vortex cells are generated (Figure 8-9b). The two corner cells further expand and a four-cell structure appears in the enclosure (Figure 8-9c). However, this four-cell structure is unstable, and the cells at diagonal positions start to merge (Figure 8-9d) and form a big counter-clockwise oriented diagonal vortex. The rolls originally at the first and the third quadrants are compressed and retreat to the corners. The symmetry of the flow structure is lost due to the interaction of vortices. The flow is then characterized by the alternative formation of the diagonal cell through the expanding and merging of corner cells (Figure 8-9e-h).

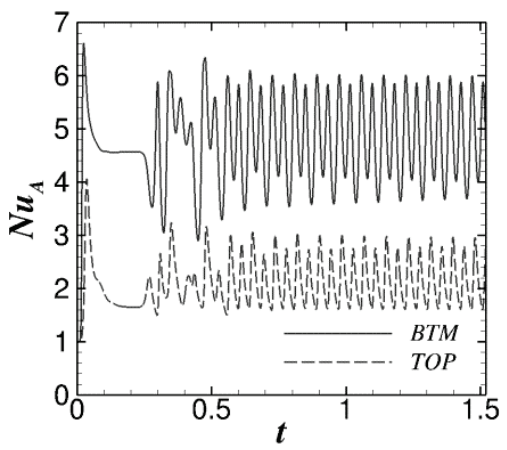

(a)

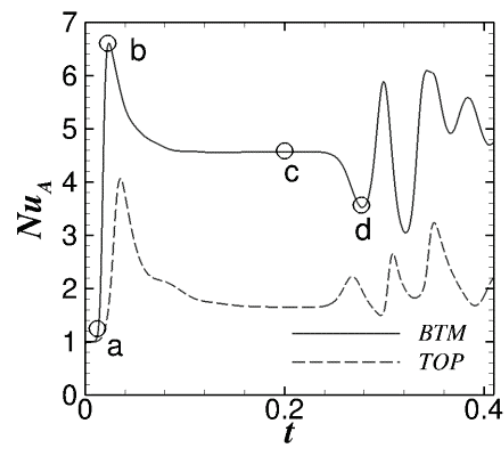

(b)

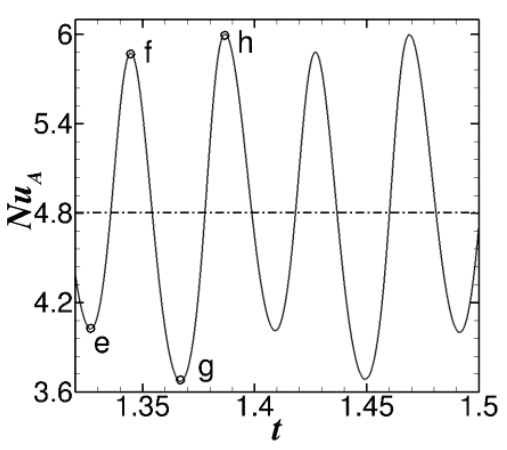

(c)

Figure 8-8: $N u_{A}$ at $\operatorname{Pr}=0.71$ and $\operatorname{Ra}=10^{5}$

(Shown: with one inserted cylinder. (a) Time course of Nusselt number at the hot and cold surfaces. (b) Transient state (c) Periodic state.) 

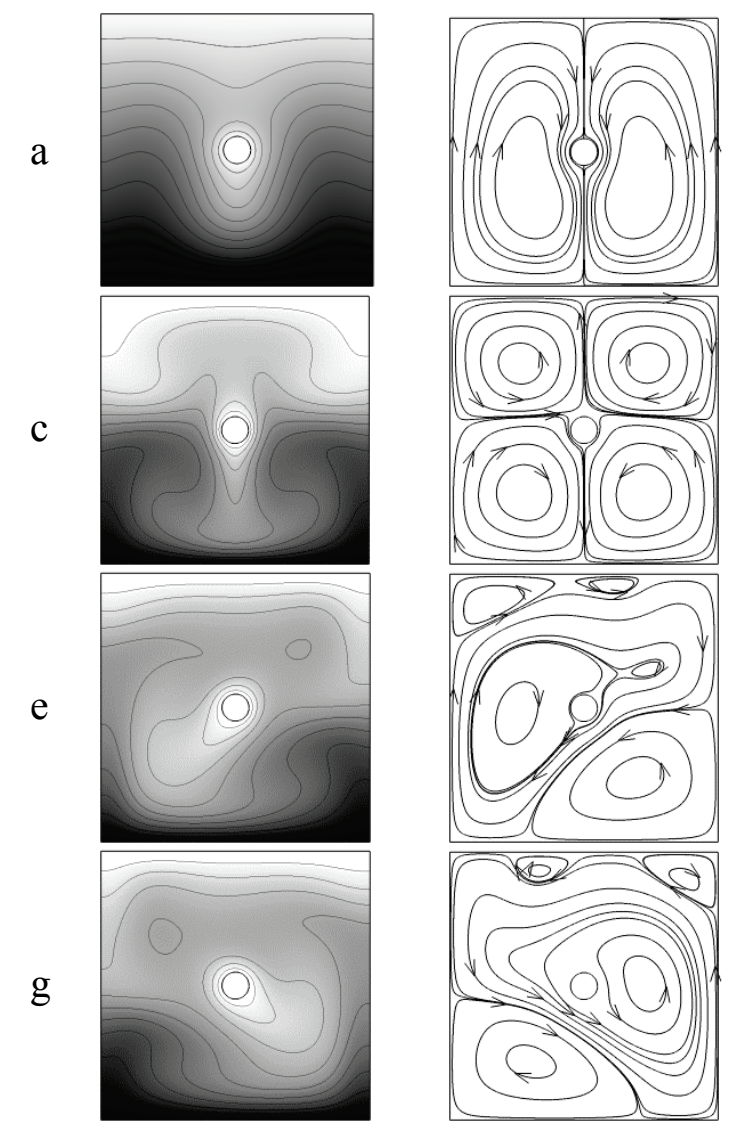

$\mathrm{b}$

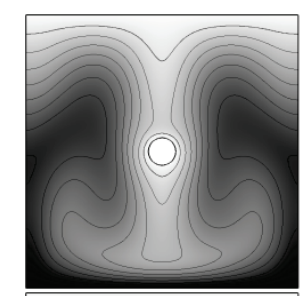

d

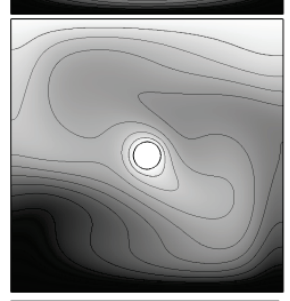

$\mathrm{h}$
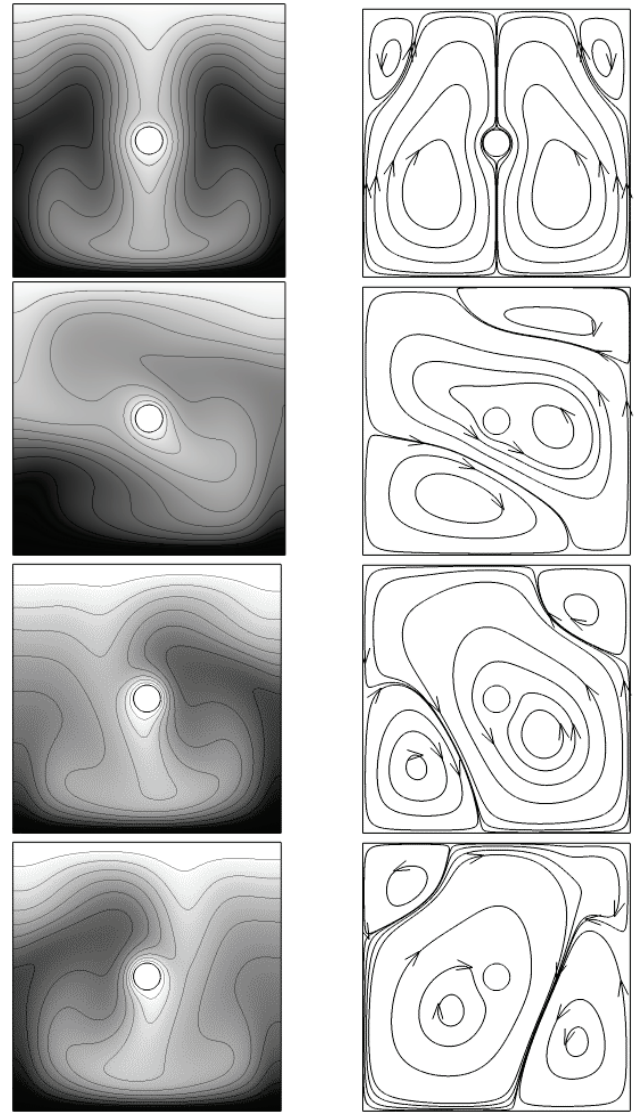

Figure 8-9: Isotherms and Streamlines at $\mathrm{Pr}=0.71$ and $\mathrm{Ra}=10^{5}$, with One Inserted Cylinder

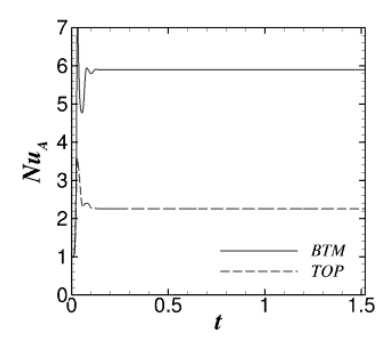

(a) $N u_{A}$

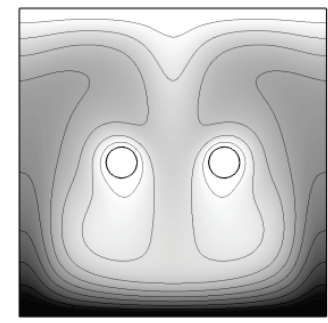

(b) Isotherms

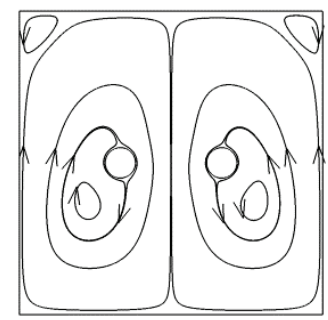

(c) Streamlines

Figure 8-10: $N u_{A}$ and Isotherms at $\operatorname{Pr}=0.71$ and $\operatorname{Ra}=10^{5}$, with Two Inserted Cylinders

In the case of two cylinders inserted into the enclosure with $\operatorname{Pr}=0.71$ and $R a=10^{5}$, the flow in the container is stabilized after a short transient process (Figure 8-10).

Symmetric bicellular flow is formed as a final state, with only two small cells at the top corners. The surface-averaged Nusselt number on the hot surface remains at a constant, with a higher value than that in the one-cylinder case. When three cylinders are placed in the enclosure, periodic solution is obtained (Figure 8-12), after a longer transient process compared to that in the reference and the one-cylinder cases. The time course of $N u_{A}$, and its variation in the transient process and the periodic state are shown inFigure 8-12. The temperature profiles and vortex structures in the transient process are presented in Figure 8-12(a) - (h), in which more complex vortex structure is formed in transient process, compared to that in the one-cylinder case. But

DISTRIBUTION STATEMENT A: Approved for public release. Distribution is unlimited. 
similarly, the symmetry of flow structure is lost as a result of the merging of diagonal cells (Figure 8-12f, g). Eventually a bicellular structure develops in the enclosure (Figure 8-12h, i, j), however, the two cells are asymmetric around the vertical center line of the enclosure, unlike the case of $R a$ $=10^{4}$. Considered both one-cylinder and three-cylinder cases, the oscillation of the heat convection is induced by the flow past over the center cylinder.

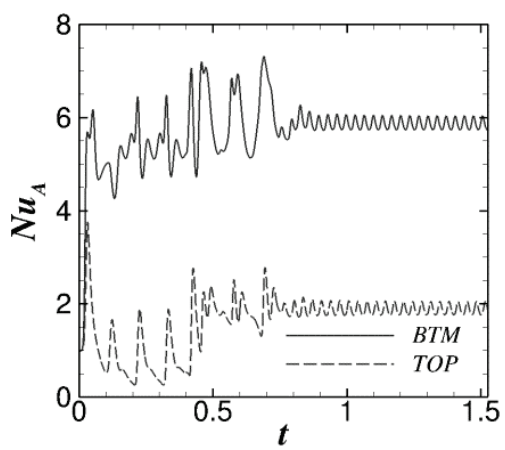

(a)

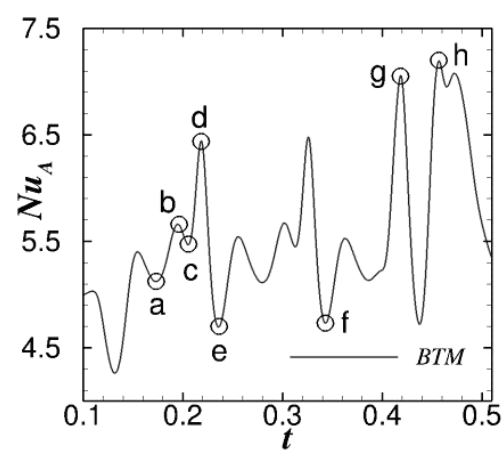

(b)

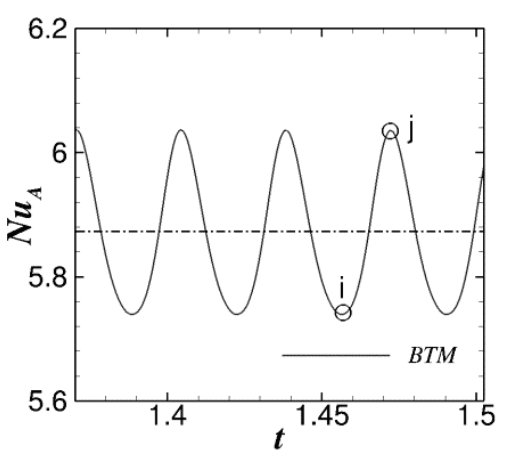

(c)

Figure 8-11: $N u_{A}$ at $\operatorname{Pr}=0.71$ and $\operatorname{Ra}=10^{5}$, with Three Inserted Cylinders

((a) Time course of Nusselt number at the hot and cold surfaces. (b) Transient state (c) Periodic state (the dash-dot line indicates the time average).) 

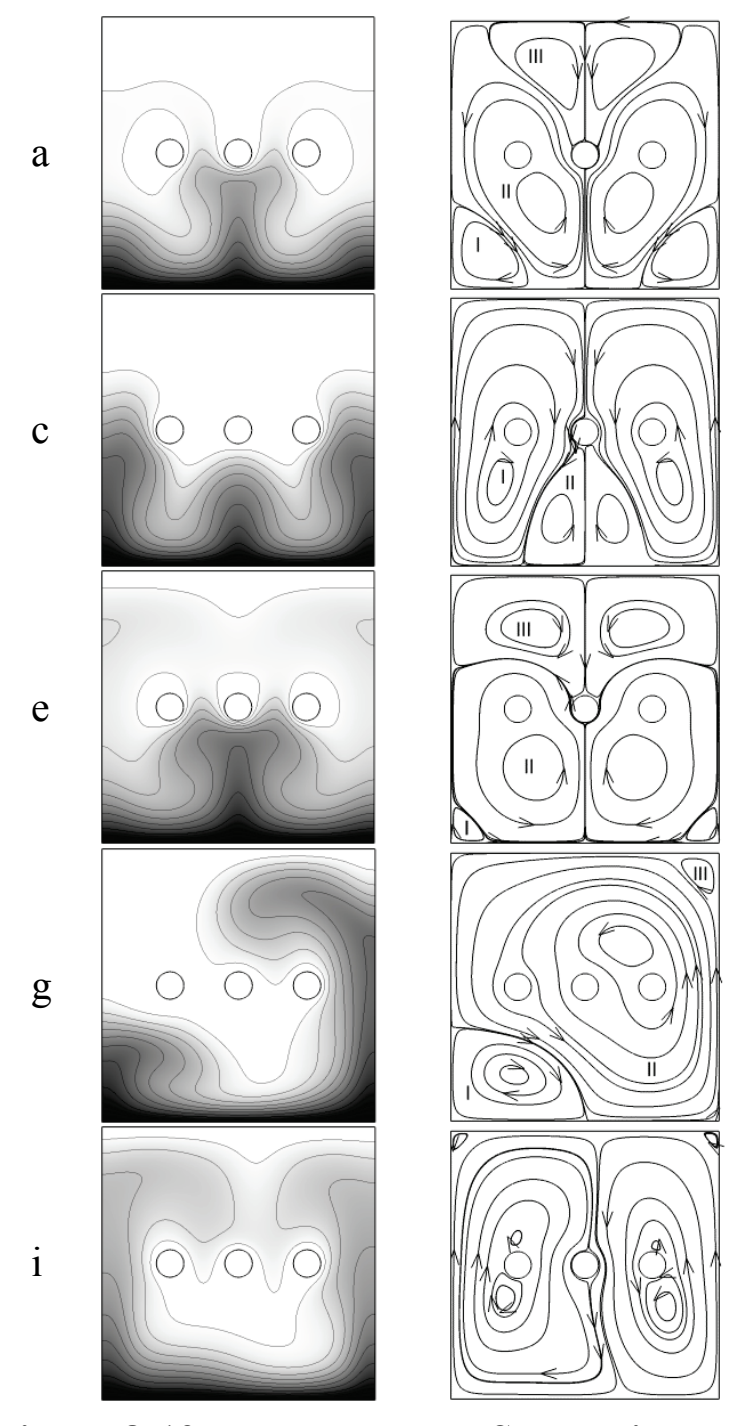

$\mathrm{b}$

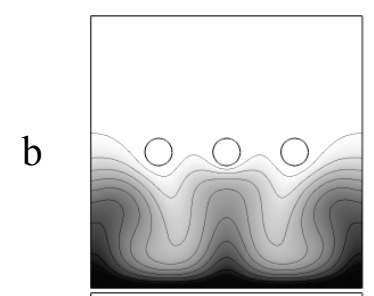

d

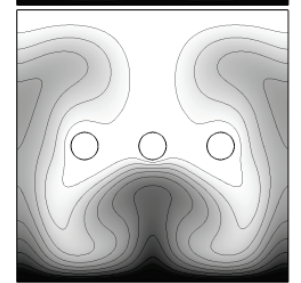

$\mathrm{h}$

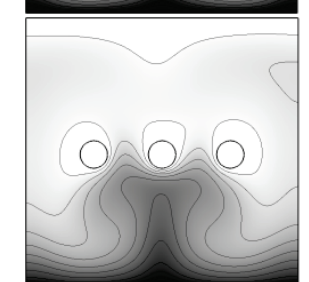

h
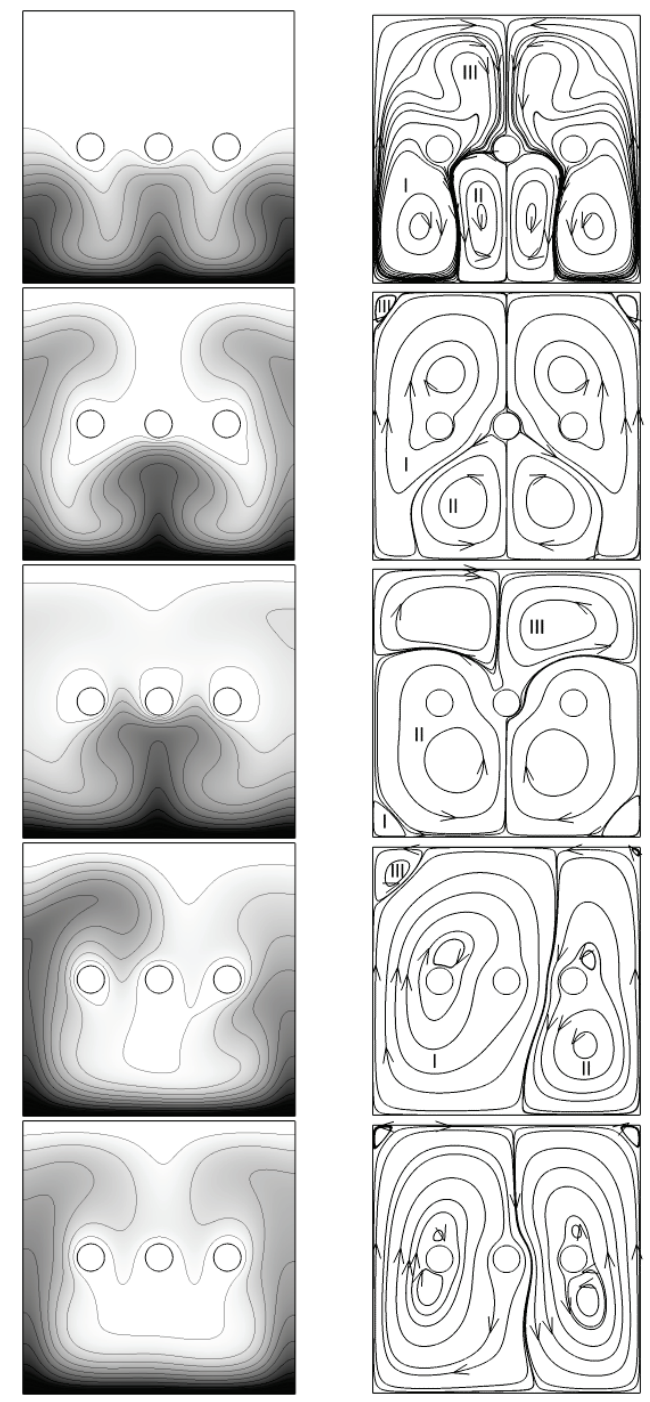

Figure 8-12: Isotherms and Streamlines at $\mathrm{Pr}=0.71$ and $\mathrm{Ra}=10^{5}$, with Three Inserted Cylinders

\subsubsection{4 $\operatorname{Pr}=\mathbf{0 . 7 1}, \mathrm{Ra}=10^{6}$}

In an empty enclosure, as the Rayleigh number increases to $10^{6}$, a periodic solution is obtained (Figure 8-13 a and b). Note $N u_{A}$ at the top and bottom surfaces are not instantaneously equal in this case, instead, they are identical in a sense of time average. Similar phenomena of $N u_{A}$ is also recently reported in a square container with a thin depth (1/10) at $\operatorname{Pr}=0.786$ and $\operatorname{Ra}=10^{6}$ [234]. Figure 8-13b shows that the surface-averaged Nusselt number at the top and bottom surfaces almost have a phase difference of $180^{\circ}$. The Nusselt number distribution along the hot surface at the maximum and minimum extremes shows the same profile, but with the different peak values as shown (Figure 8-13c). Figure 8-13d and e present the flow structure at the instant of minimum and the maximum $N u_{A}$ on the bottom surface. The size of the two vertically aligned principal vortices, especially the size of the cell in the lower bottom, affects the heat convection on the bottom surface quite a bit. The oscillation of $N u_{A}$ is induced by the expansion and contraction of the two vortex cells. Since the expansion of one cell is associated with the contraction of the other, $N u_{A}$ on hot and cold surfaces are out of phase for about a half cycle. As the bottom cell is compressed, $N u_{A}$ at the hot surface is minimum, corresponds to the label a in Figure 8-13b. As the

DISTRIBUTION STATEMENT A: Approved for public release. Distribution is unlimited. 
bottom cell expanding in the vertical direction, $N u_{A}$ increases and reaches its maximum value (label $\mathrm{b}$ in Figure 8-13b). Figure 8-13f presents the time-averaged isotherms in the enclosure. Due to the vertical aligned vortex structure, the lower part of the enclosure is mainly dominated by hot temperature.

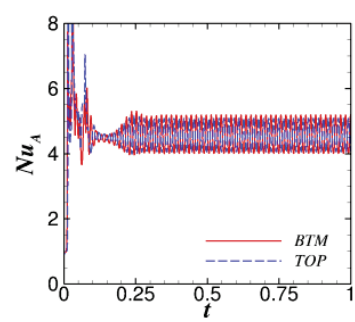

a

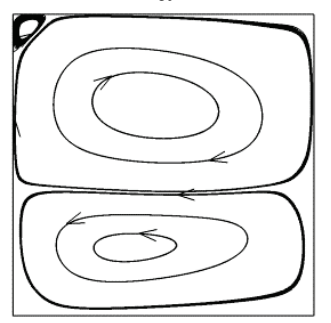

d

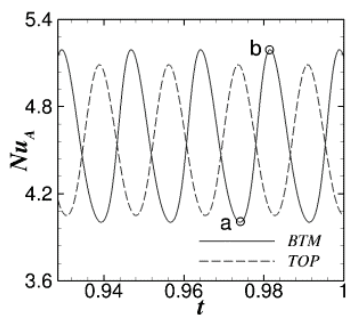

$\mathrm{b}$

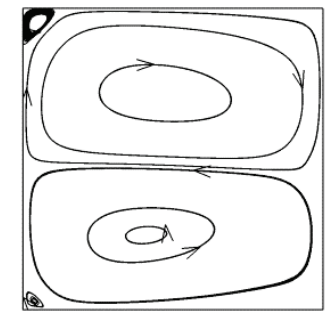

e

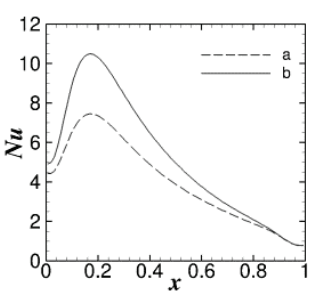

C

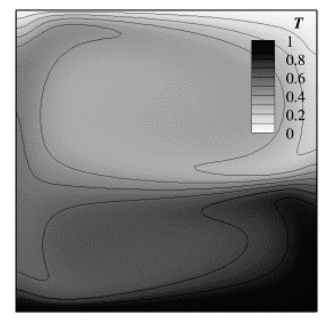

$\mathrm{f}$

Figure 8-13: Reference Case of $\operatorname{Pr}=0.71$ and $\operatorname{Ra}=10^{6}$

((a) Surface-averaged Nusselt number at the bottom and top surfaces. (b) $N u_{A}$ in periodic state. (c) Nusselt number distribution along the hot surface at time instants $a$ and $b$. (d) Streamlines at point $a$. (e) Streamlines at point $b .(f)$ Time-averaged temperature contour.)

As cylinders inserted in the enclosure, the flow inside is totally disturbed and unsteady. The surface-averaged Nusselt numbers show aperiodic convection at the bottom and top surfaces. The flow in the enclosure shows very complex vortices generation and interaction, and it is hard to categorize the motion of the cell structure, even in a sense of time average. Therefore, we here only present the surface-averaged Nusselt number $N u_{A}$ at the bottom and top surfaces (Figure 8-14).
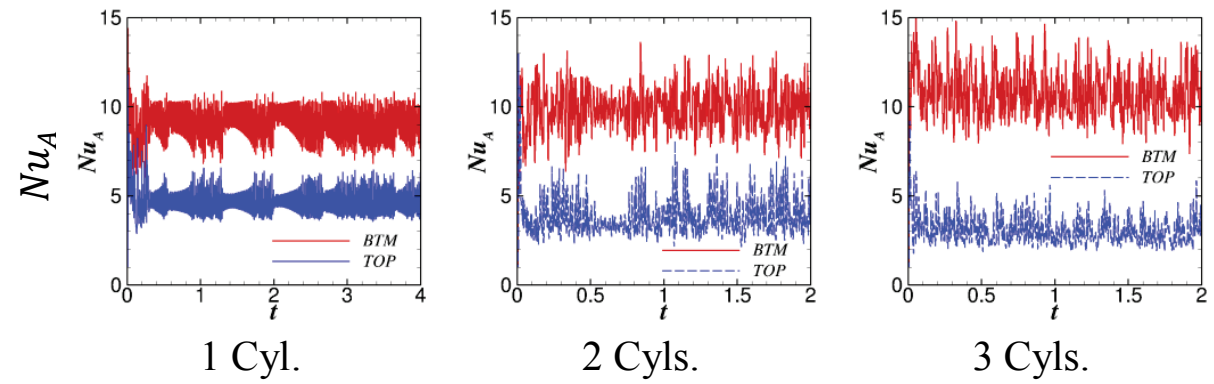

Figure 8-14: Surface-averaged Nusselt Number at $\operatorname{Pr}=0.71$ and $\operatorname{Ra}=10^{6}$

Table 8-2 summarized the surface-averaged Nusselt number $N u_{A}$ at the bottom surface for $\operatorname{Pr}=0.71$, at various Rayleigh numbers. At low $R a$, in which conduction dominates, the threecylinder case improves the heat transfer at the enclosure bottom by a factor of 2 . Further increasing the number of cold cylinders can only bring minor improvement, since the heat transfer process is now already constrained in the lower half enclosure. At $R a=10^{4}$ and $R a=10^{5}$, the tow-cylinder

DISTRIBUTION STATEMENT A: Approved for public release. Distribution is unlimited. 
case gives the best enhancement on $N u_{A}$ at the bottom surface. $N u_{A}$ at the three-cylinder case at these two Rayleigh number is actually reduced, due to the suppression of the heat convection. As the Rayleigh number is increased to $10^{6}$, one cylinder can totally disturb the two-cell flow structure as obtained in the reference case, and $N u_{A}$ at the hot surface is almost doubled. The two-cylinder and tthree-cylinder cases can still enhance the heat transfer at the bottom by inducing strong convections nearby, but we believe the heat transfer enhancement will be saturated, and then dropped down, when heat convection in the enclosure is suppressed if more cold cylinders are inserted.

Table 8-2: $N u_{A}$ at the Hot Surface for $\operatorname{Pr}=0.71$, at Various Rayleigh Numbers

\begin{tabular}{lllll}
\hline Ra & $10^{3}$ & $10^{4}$ & $10^{5}$ & $10^{6}$ \\
\hline Ref. & 0.991 & 1.713 & 4.017 & $4.535 \pm 0.612$ \\
1 Cyl. & 1.581 & 2.281 & $4.805 \pm 0.771$ & $8.972 \pm 0.699$ \\
2 Cyls. & 1.874 & 2.653 & 5.899 & $9.913 \pm 0.930$ \\
3 Cyls. & 1.997 & 2.269 & $5.873 \pm 0.112$ & $10.625 \pm 0.971$ \\
\hline \hline
\end{tabular}

\subsubsection{Pr number 7.1 (Water)}

\subsubsection{1 $\operatorname{Pr}=7.1, \mathrm{Ra}=10^{5}$}

The heat transfer is principally dominated by the conduction when $R a$ is small. In the case of $R a=10^{5}$, convection plays a major role in the heat transfer in the enclosure. In the reference case, the thermal plume rises from the middle of the hot surface due to the formation of the two vortices. This result is consistent with the vortex structure discussed in Ref. [254] at this Rayleigh number. The flow reaches the steady state, and the surface-averaged Nusselt number at the hot surface stabilized at a constant value around 2.7 after the initial oscillation fades out. When one cylinder is inserted at the center of the enclosure, the temperature distribution in the enclosure is inverted, compared to that in the reference case. The temperature gradient in the middle region of the hot surface is increased. From the time courses of $N u_{A}$, we can see that the two-cylinder and three-cylinder case comparably enhance the heat transfer process on the hot surface. The oscillation in the transient process of the three-cylinder case is, however, smaller than that in the two-cylinder case. Furthering increasing the number of cylinder will totally smear of the oscillation in the transient process, and $N u_{A}$ monotonically approaches to the steady value. 

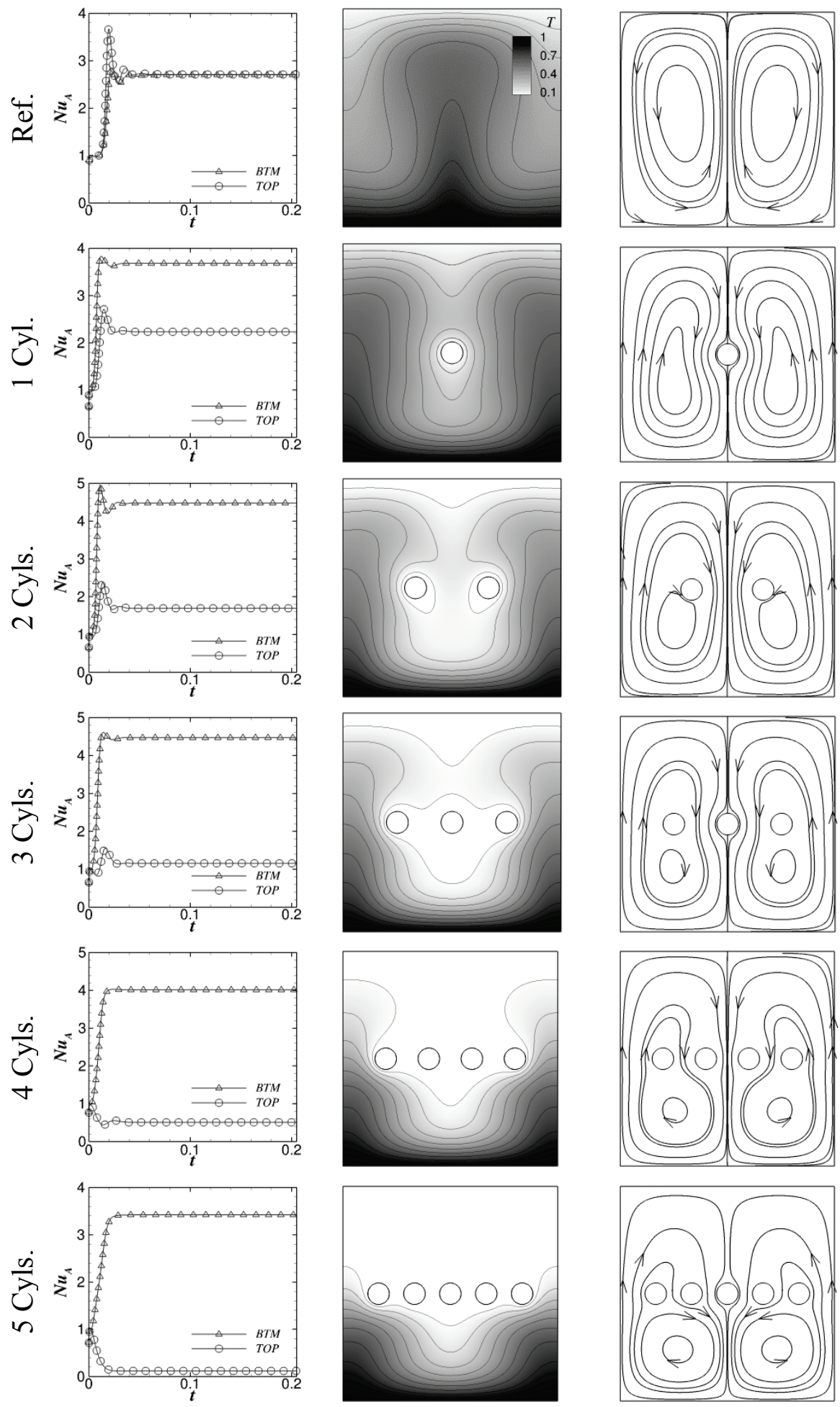

(a) $N u_{A}$

(b) Isotherms

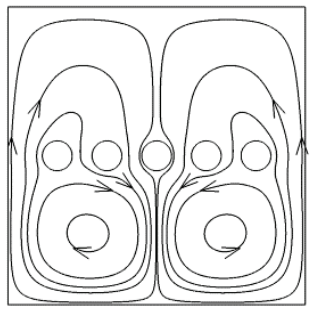

(c) Streamlines

Figure 8-15: $\mathbf{N} \boldsymbol{u}_{\boldsymbol{A}}$ Isotherms and Streamlines for $\mathrm{Pr}=\mathbf{7 . 1}$ and $\mathbf{R a}=\mathbf{1 0}^{5}$

(Simulations conducted up to $t=1$. Results of $t \leq 0.2$ are shown for a clearer view of the transient process.) 
Figure 8-16 presents the Nusselt number distribution along the hot and the cold surfaces at $R a=10^{5}$. In the empty enclosure, the convection in the central region of the bottom (hot) surface is small compared to the region near edges, while the convection in the mid-region of top surface is higher than that at corner regions. As code cylinders are inserted, convection in the central region of the bottom surface is highly enhanced. Two and three cylinders shows the similar effect on the Nusselt number distribution, and further increasing number of cylinders actually inversely affect the heat convection on the bottom. On the cold surface, heat transfer is reduced when cold cylinders are placed. As more cylinders inserted, the top surface is more likely shielded by the cylinders, and the heat transfer process is more constrained in the lower half of the enclosure.

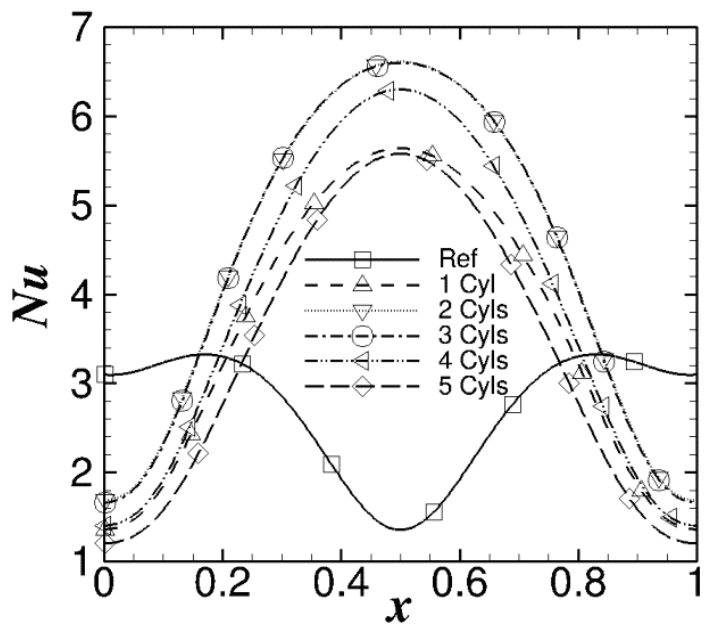

(a) Bottom surface

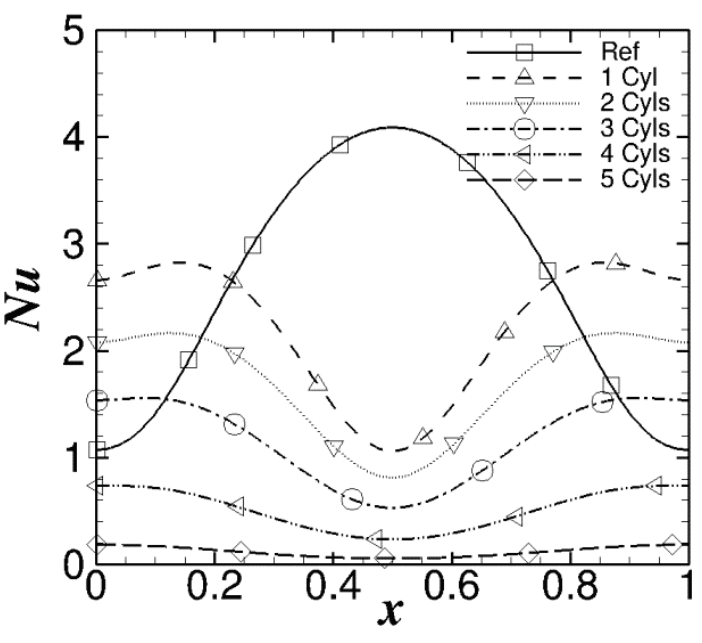

(b) Top surface

Figure 8-16: Nusselt Number Distribution at the Bottom (hot) and Top (cold) Surfaces

$$
\left(\operatorname{Pr}=7.1, R a=10^{5} .\right)
$$

\subsubsection{2 $\operatorname{Pr}=$ 7.1, $R a=10^{6}$}

Figure 8-17 shows the Nusselt number for the reference case of $\operatorname{Pr}=7.1, R a=10^{6}$. The surface averaged Nusselt number $N u_{A}$ shows periodic oscillation after the transient process fading out. The details of the oscillation are shown in Figure 8-17(b). Again, $N u_{A}$ at the hot and cold surfaces are identical in the sense of time average, instead of instantaneously. The Nusselt number distributions along the bottom surface are drawn in Figure 8-17 for several time instants selected at minimum, mean, and maximum $N u_{A}$. The corresponding isotherms and streamlines are presented in Figure 8-18. In Figure 8-18, the flow structure is characterized by a single rolling cell, whose center itself is circulating in a clockwise direction. 


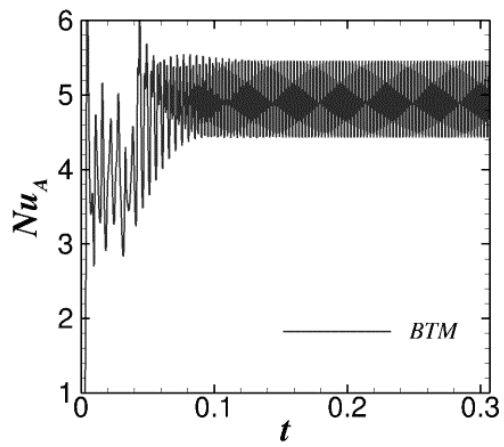

(a)

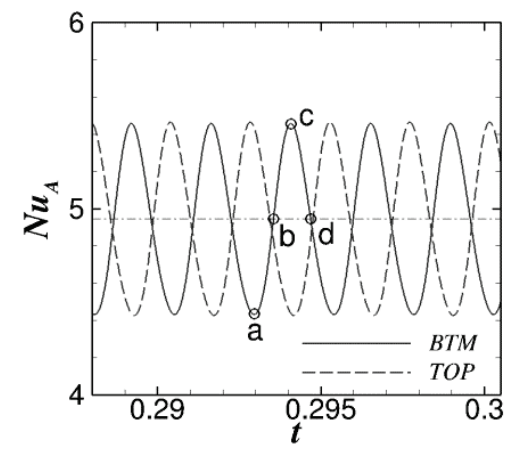

(b)

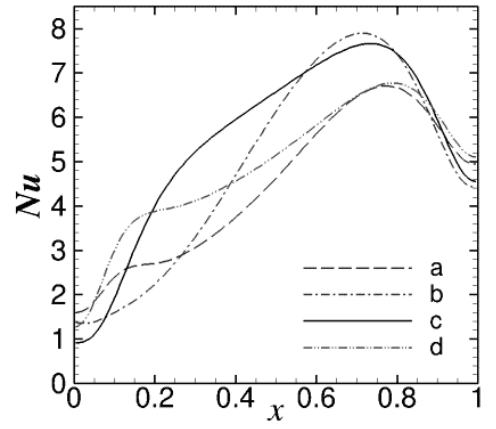

(c)

Figure 8-17: $N u_{A}$ at the Bottom (hot) and Top (cold) Wall

$\left(\operatorname{Pr}=7.1, \mathrm{R} a=10^{6}\right.$. The simulation was conducted until $t=1$, however, results of only $t<0.3$ are shown for a clearer view.)

a

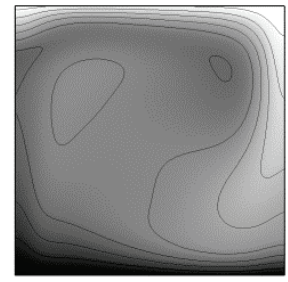

c

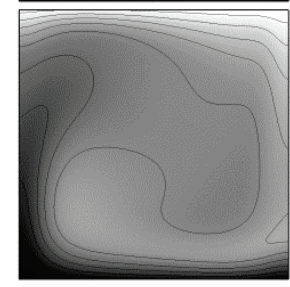

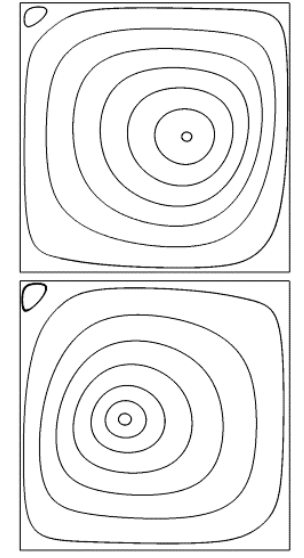

$\mathrm{b}$

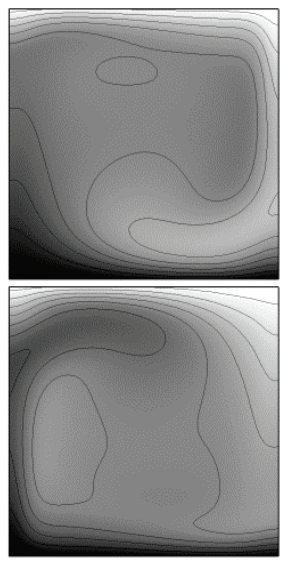

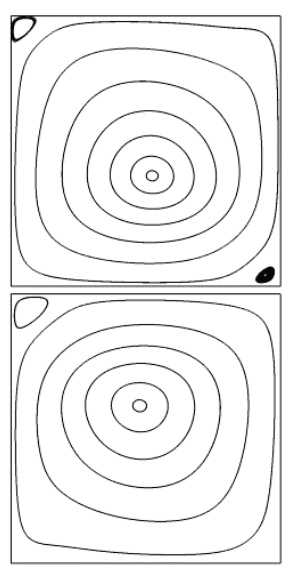

Figure 8-18: Isotherms and Streamlines for the Reference Case of $\operatorname{Pr}=7.1, \operatorname{Ra}=10^{6}$

When one cylinder is inserted in the enclosure, the transient process is greatly reduced and a quasi-periodic state is quickly reached (Figure 8-19).

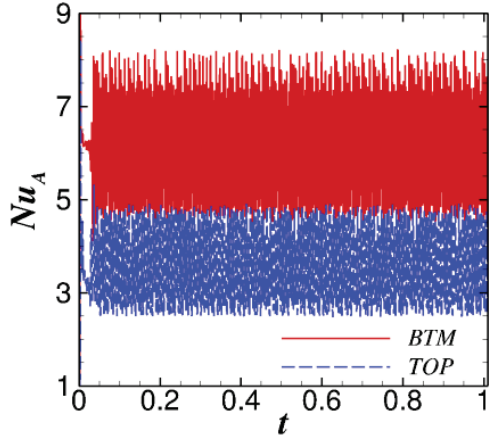

(a)

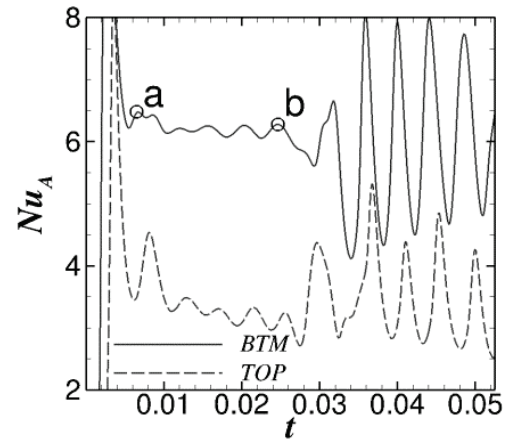

(b)

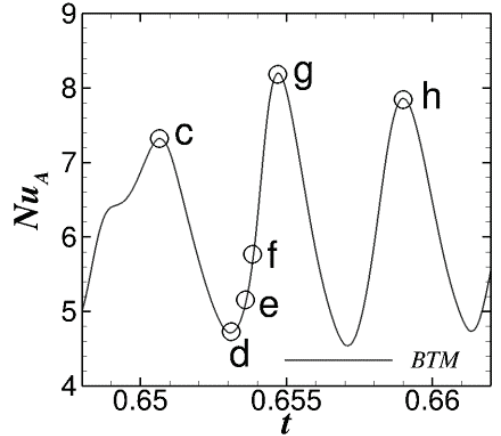

(c)

Figure 8-19: Nusselt Number at the Bottom (hot) and Top (cold) Wall, One Cylinder in a Cavity, $\operatorname{Pr}=7.1, \operatorname{Ra}=10^{6}$

((a) Time course of Nusselt number (b) transient process (c) quasi-periodic state.) 
The zoomed-in $N u_{A}$ at the transient process and at the quasi-periodic state are shown in Figure 8-19 (b) and (c) respectively. The corresponding flow patter and thermal contour are presented in Figure 8-20.
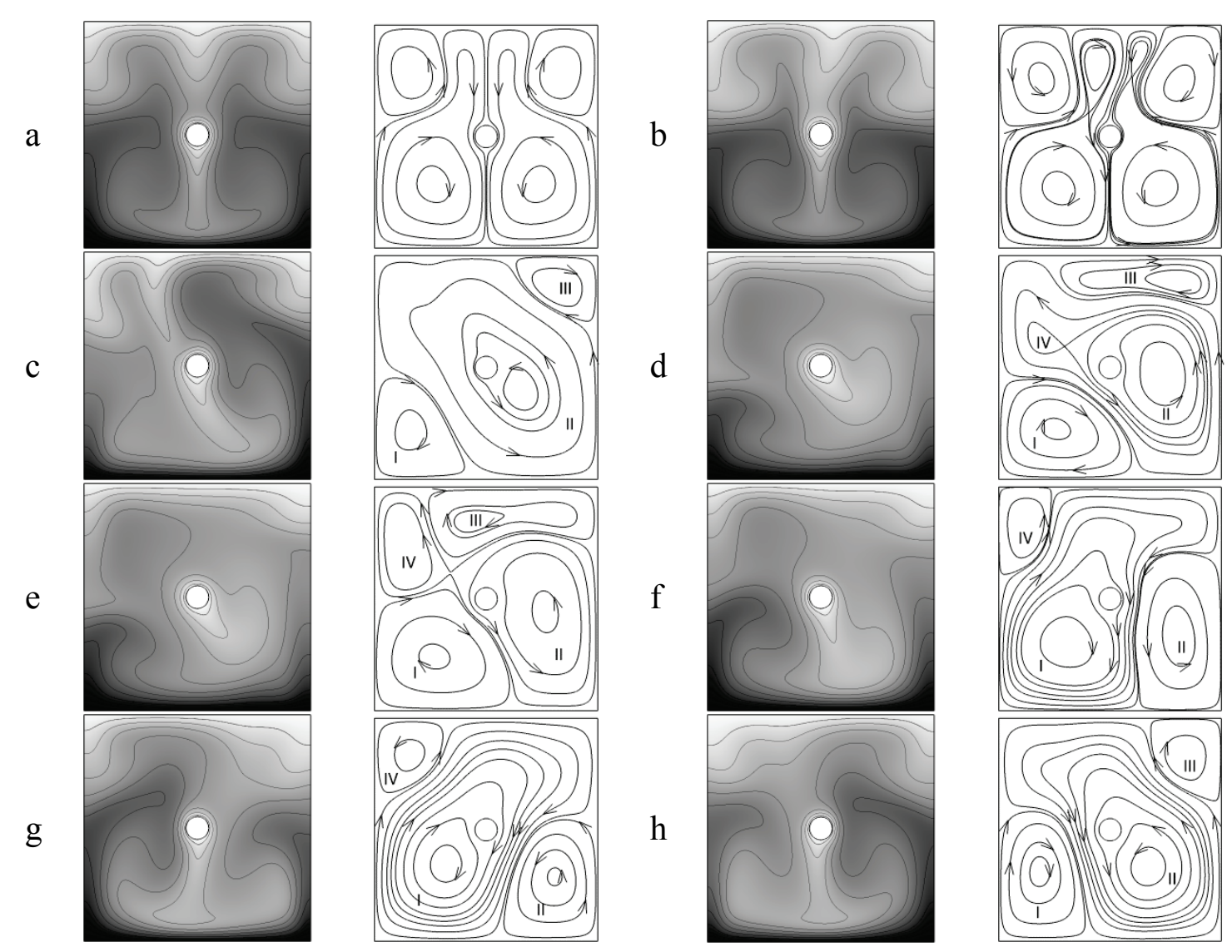

Figure 8-20: Isotherms and Streamlines in a One-cylinder Enclosure, $\operatorname{Pr}=7.1, \mathrm{Ra}=10^{6}$

In the transient process, the flow pattern is initially symmetric around the vertical centerline $\mathrm{x}=0.5$ (Figure 8-20). However, this symmetric pattern is unstable, and perturbation grows as the vortices in the upper half enclosure starts to deform and merge. The flow structure is characterized by two corner vortices (e.g., I and III in Figure 8-20c) and a diagonal vortex (vortex II). As the corner vortex grows, the diagonal vortex is squeezed and split two vortices (II and IV Figure 8-20d). The vortices I and III then keep growing and merges to form a new diagonal vortex (I in Figure 8-20(f) and (g)), but aligned with an opposite direction as compared to the one in Figure 8-20(c). The corner vortices II and IV then starts to grow and another half cycle starts.

When two cylinders are placed in the enclosure, the surface-averaged Nusselt number $N u_{A}$ is more separated (Figure 8-21), as compared to that in the case of one cylinder. $N u_{A}$ is aperiodic, indicating the flow is strongly disturbed by the two cylinders. However, the time-averaged flow field and temperature field basically show a symmetry pattern around the vertical centerline of the enclosure. As more cold cylinders are inserted, the flow is actually stabilized, and steady solution is approached quickly. 

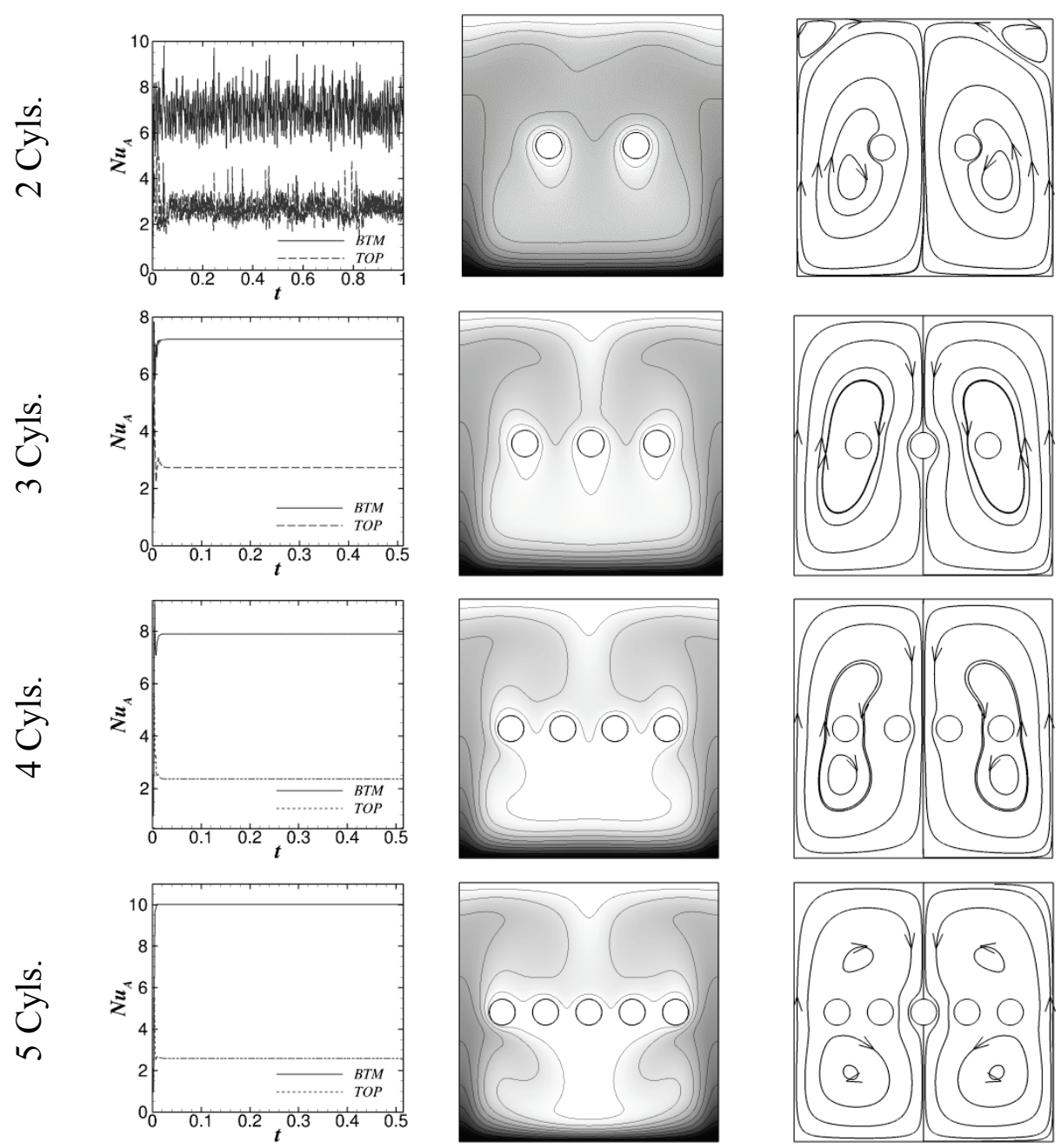

(a) $N u_{A}$

(b) Isotherms

(c) Streamlines

Figure 8-21: $N u_{A}$ and Isotherm at $\operatorname{Pr}=7.1$ and $\operatorname{Ra}=10^{6}$

(The two-cylinder case shows oscillation in $\mathbf{N u}_{\boldsymbol{A}}$. The isotherms and streamlines for the 2-Cyls. case are time-averaged results from $t=0.8$ to $t=1.0$.)

Table 8-3: Nusselt Number at the Hot Surface for $\operatorname{Pr}=7.1$, at Various Ra, and Number of Cylinders

\begin{tabular}{llll}
\hline$R a$ & $10^{4}$ & $10^{5}$ & $10^{6}$ \\
\hline Ref & 0.992 & 2.707 & $4.945 \pm 0.512$ \\
1 Cyl. & 1.573 & 3.685 & $6.188 \pm 1.041$ \\
2 Cyls. & 1.847 & 4.479 & $6.935 \pm 0.581$ \\
3 Cyls. & 1.974 & 4.469 & 7.220 \\
4 Cyls. & 2.043 & 4.014 & 7.899 \\
5 Cyls. & 2.083 & 3.421 & 10.018 \\
\hline
\end{tabular}


The surface averaged Nusselt number $N u_{A}$ at the hot surface is listed in the Table 8-3 for $\operatorname{Pr}$ 7.1. At $R a=10^{4}$, the conduction dominants the heat transfer process. As the number of the cylinder increases to $5, N u_{A}$ is about doubled compared to the reference case, adding the cylinders does not change the heat transfer mechanism in the low Rayleigh numbers. At $R a=10^{5}$, the twocylinder and three-cylinder cases generated comparable enhancement on the hot surface. Further inserting the cylinders inversely affect the heat convection thereby. As $R a$ is as high as $10^{6}$, the five-cylinder case produces most improvement in $N u_{A}$. We also conducted simulation for the case of seven-cylinder (not listed in the table), the value of $N u_{A}$ is lowered to 8.074.

\subsubsection{Small Pr Number $(\mathrm{Pr}=\mathbf{0}$.021)}

Liquid metals usually possess small Prandtl numbers [255]. Here we selected $\operatorname{Pr}=0.021$ (liquid gallium) as an example to study its heat transfer properties in an enclosure with vertical temperature gradient. The details of steady convective flows in a cavity containing liquid gallium was studied by Braunsfurth et al. [256], in which a horizontal temperature gradient was applied through the cavity. The onset of oscillatory convection in a horizontal cavity was studied by Hof et al. [257]. When low Prandtl number medium is used, the thermal diffusivity is far greater than the fluid viscosity, and the heat transfer is dominated by the heat conduction, especially in the range of small and medium Rayleigh numbers [258].

\subsubsection{1 $P r=0.021$, Small $R a$}

In our study of gallium, the heat transfer in an empty enclosure is dominated by the conduction when side walls are adiabatic (Table 8-4), in the Ra range of $10^{3} \sim 10^{4}$. At low Rayleigh number such as $R a=10^{3}$, heat transfer is dominated by the conduction. The isotherm is similar to that of $\operatorname{Pr}=0.71$, and $R a=10^{3}$. For the case of $R a=10^{4}$, as one or two cold cylinders are inserted, the heat transfer in the enclosure is more convection-dominated. The flow is symmetric and is characterized by the two principal vortex structures. Although the secondary cells exist at the corners, they may not help much in the heat transfer. As three cold cylinders are placed, the flow in the enclosure is stabilized. The convection effect is diminished, and the heat transfer is mainly occurs between the bottom surface and the inserted three cylinders. From Figure 8-22 we can see that the two-cylinder case approaches the stead state faster than the one-cylinder and three-cylinder cases. This could be ascribed to the generated disturbance as flow passing over the cylinder at the enclosure center. 

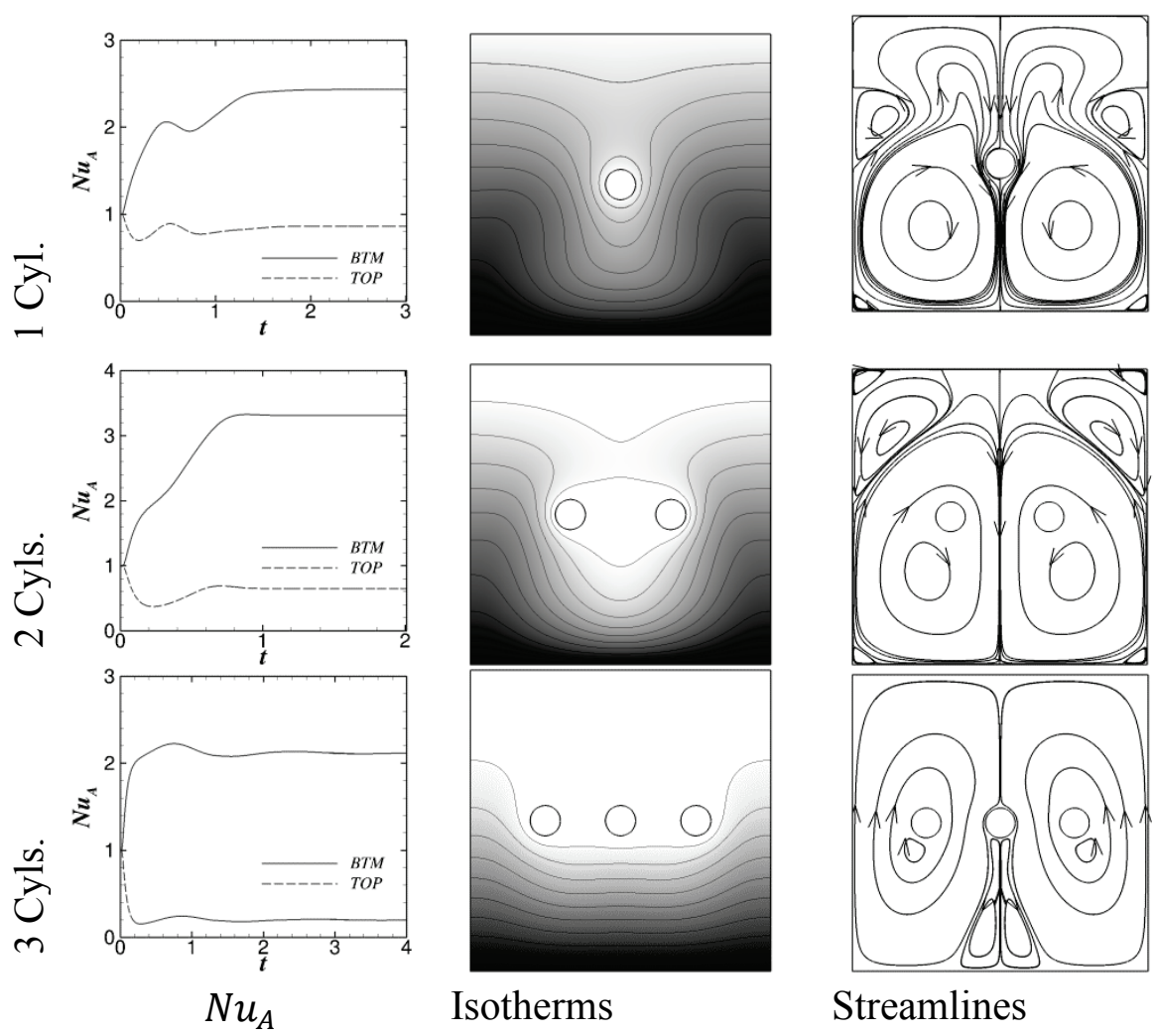

Isotherms

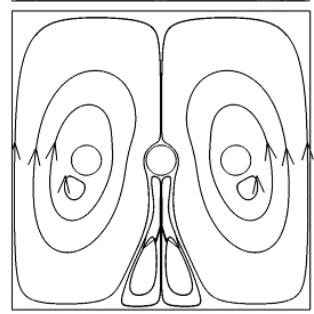

Streamlines

Figure 8-22: Surface-averaged Nusselt Number, Isotherms, and Streamlines at $\mathrm{Pr}=\mathbf{0 . 0 2 1}$, $R a=10^{4}$

\subsubsection{2 $P r=0.021, R a=10^{5}$}

Figure 8-23 presents the surface-averaged Nusselt number $N u_{A}$ at the bottom in an empty enclosure when $P r=0.021$ and $R a=10^{5}$. It can be seen that the heat transfer initially reaches a quasi-steady state, then it develops to a periodic state. Four cells are generated in the quasi-steady state, and the flow structure is symmetric around the center of the enclosure (Figure 8-23a). The diagonal vortices than start to merge and form a big one (Figure 8-23b). Eventually, the flow structure is characterized by a unicellular circulation, with four small vortices at corners.

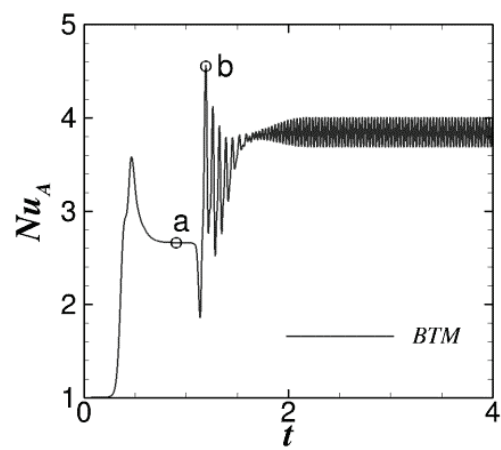

(a)

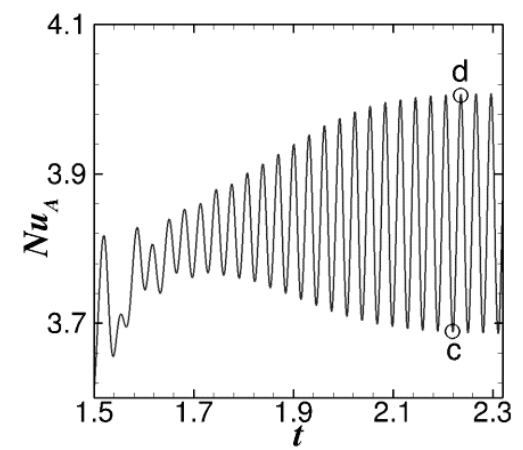

(b)

Figure 8-23: $\mathrm{Nu}_{\boldsymbol{A}}$ at the Bottom Surface for the Reference Case at $\mathrm{Pr}=\mathbf{0 . 0 2 1}$ and $\mathrm{Ra}=\mathbf{1 0}^{5}$

((a) Time courses of the Nusselt number; (b) Transient state; (c) Periodic state.) 


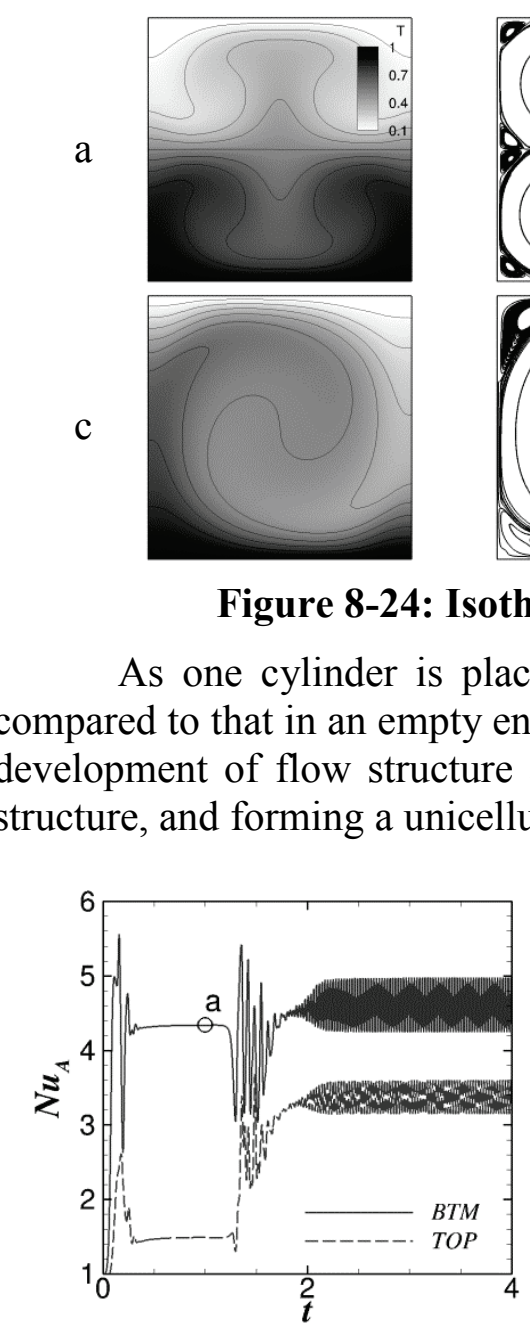

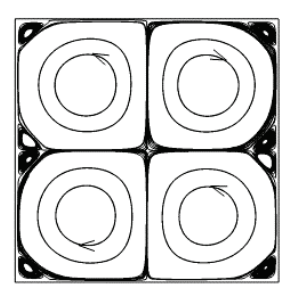

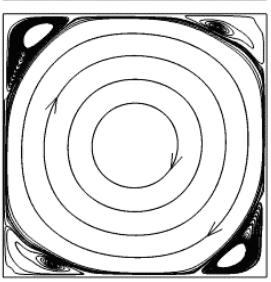

$\mathrm{b}$

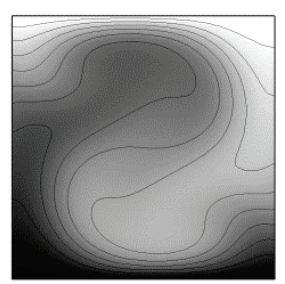

d

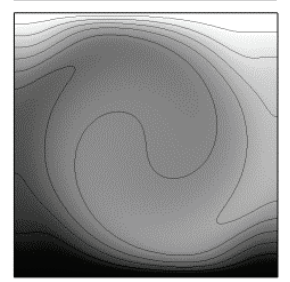

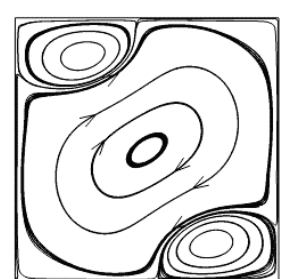

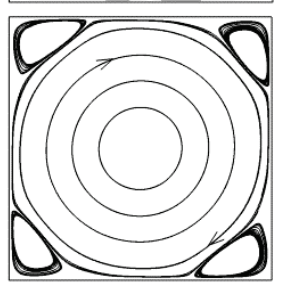

Figure 8-24: Isotherms and Streamlines at $P r=0.021$ and $R a=10^{5}$ compared to that in an development of flow structure also presents a similar trend, staring with an unstable four-cell structure, and forming a unicellular structure with four corner cells eventually.

(a)

Figure 8-25: $N u_{A}$ at the Bott

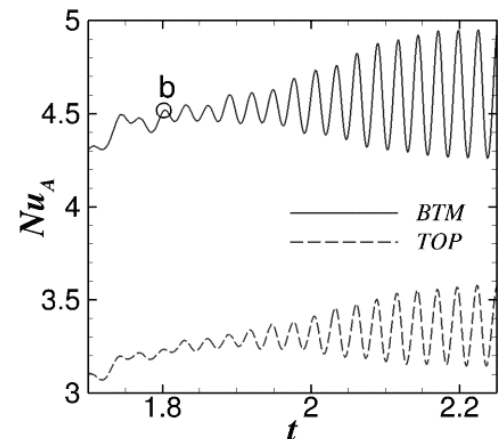

(b)

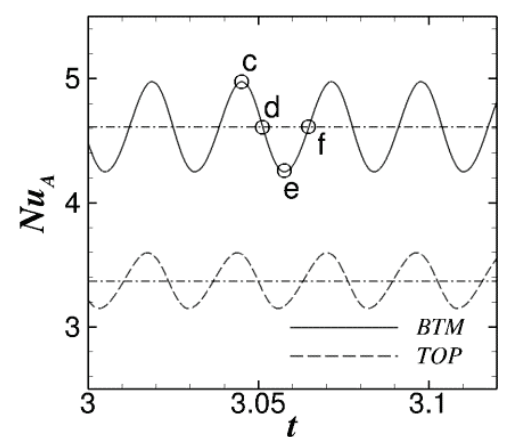

(c)

Figure 8-25: $\mathbf{N} \boldsymbol{u}_{\boldsymbol{A}}$ at the Bottom and Top Surfaces

( $P r=0.021$ and $R a=10^{5}$, with one cylinder in the center of the enclosure. (a) Time courses of the Nusselt number; (b) Transient state; (c) Periodic state.) 

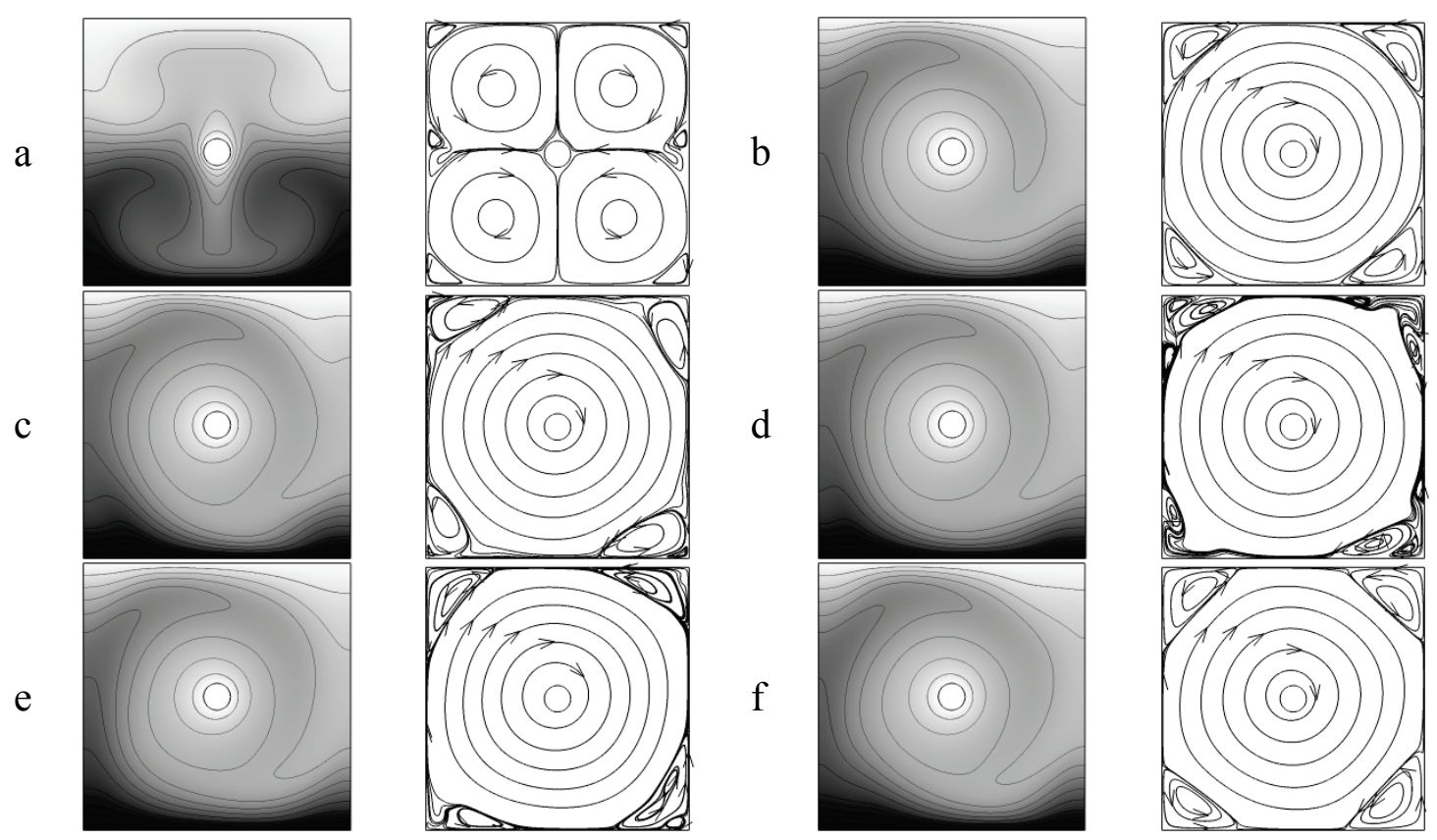

Figure 8-26: $N u$ and Isotherm at $P r=0.021$ and $R a=10^{5}$

(The transient state seems interesting.)

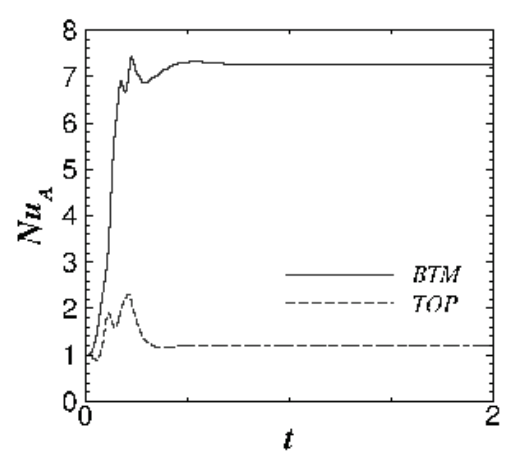

(a)

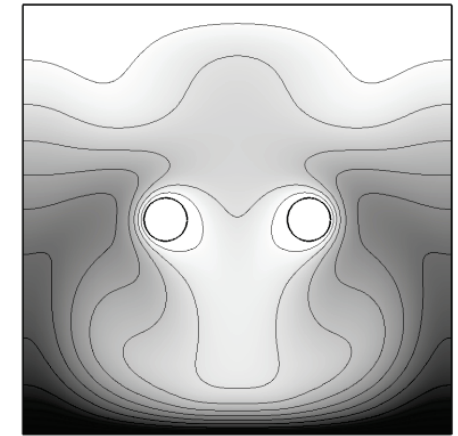

(b)

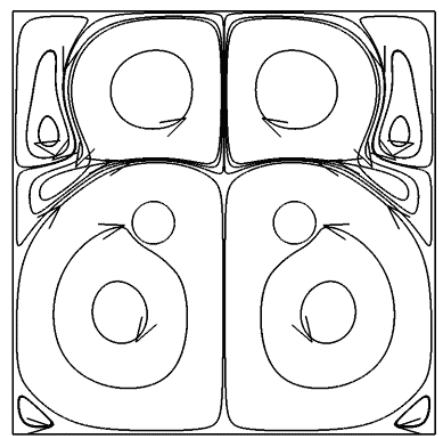

(c)

Figure 8-27: $\boldsymbol{N} \boldsymbol{u}_{\boldsymbol{A}}$ at the Bottom and Top Surfaces

( $P r=0.021$ and $R a=10^{5}$, with two cylinders in the center.)

When two cylinders are placed in the enclosure, the steady state is easily approached, and the flow structure is symmetric around the vertical centerline $(x=0.5)$ (Figure 8-27). 


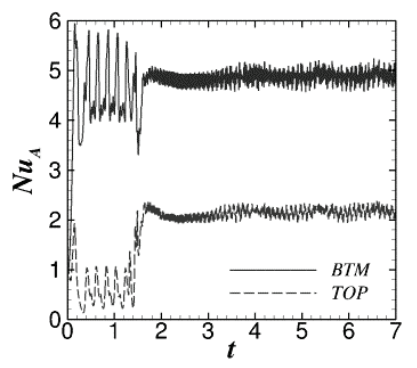

(a)

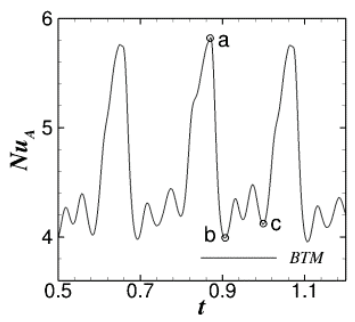

(b)

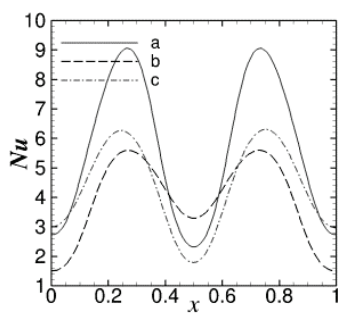

(c)

Figure 8-28: $\operatorname{Pr}=0.021$ and $R a=10^{5}$

((a) Surface-averaged Nusselt number $N u_{A}$; (b) Surface-averaged Nusselt number in stage one; (c) Nusselt number distribution along the hot bottom. Three cylinders are in the enclosure.)

Similar to the reference case and the one-cylinder case, the three-cylinder case also shows a two-stage process as presented Figure 8-28(a) by the surface-averaged Nusselt number. Figure $8-28$ (b) shows a section in the first state. The time instants for the Nusselt number distribution in Figure 8-28(c) and the flow in Figure 8-28 are labeled in Figure 8-28(b). In the first stage, the flow structure in the enclosure and the Nusselt number distribution on the hot bottom are almost symmetric around the centerline $x=0.5$; therefore, the Nusselt number distribution along the hot bottom shows two peaks. At time instant ' $a$ ', at which the surface-averaged Nusselt number is instantaneously high, the flow structure in each side of the enclosure is characterized by a big vortex (II), which crosses over the whole depth of the enclosure. Two relatively small vortices I and III at located at the corner and the top of the center cylinder respectively. The big vortex II is then compressed (Figure 8-29b) due to the growth of vortices I and III. In Figure 8-29 (c), vortex II was cut and split into vortices II and IV. Vortices I and III are merged to form a big vortex (for simplicity, labeled by I). The vortex II retreats to a small region under the center cylinder.
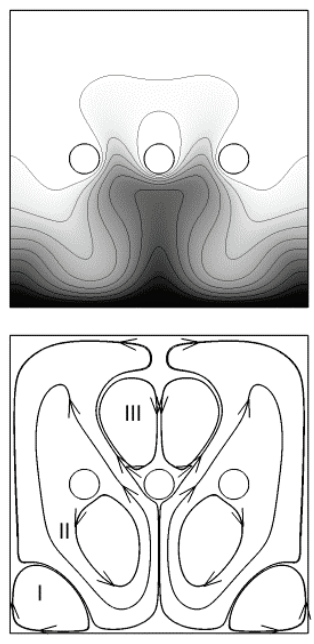

(a)
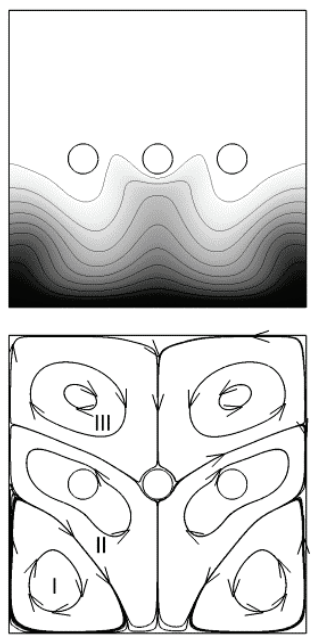

(b)
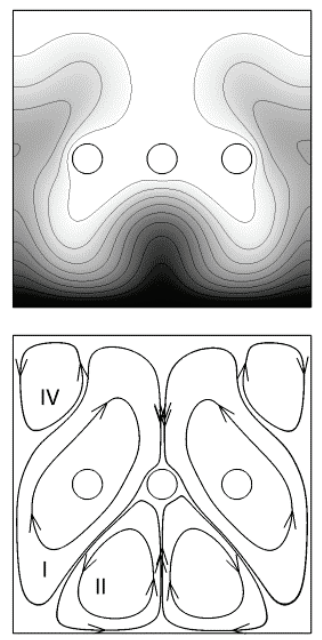

(c)

Figure 8-29: Isotherms and Flow Structure at Time Instants Corresponding to Figure 8-28, $\operatorname{Pr}=0.021$ and $R a=10^{5}$

(The time instants are labeled in Figure 8-28(b).) 


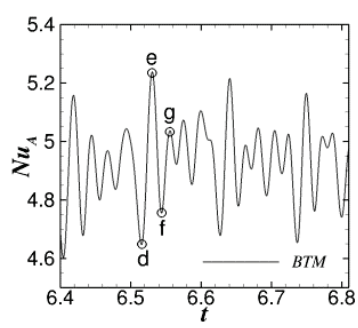

(a) $N u_{A}$

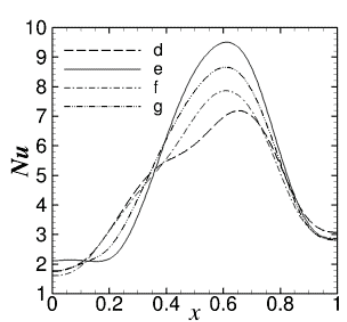

(b) $\mathrm{Nu}$

Figure 8-30: Surface-averaged $N u_{A}$ and the Nusselt Number Distribution along the Hot Surface, $P r=0.021$ and $R a=10^{5}$

(Three cylinders are in the enclosure.)

Once the symmetry is broken due to certain disturbances, new flow topology develops for the second stage, as depicted in Figure 8-31. The most distinctive flow structure is the big clockwise single cell rotating around the three cylinders. Vortex shedding is generated as the result of flow separation when the rotating flow past over the side cylinders, as shown in the region labeled by ' $\mathrm{S}$ '. The surface-averaged Nusselt number in a section of the second stage and the $N u$ distribution at the labeled points are shown in Figure 8-30. The Nusselt number along the hot bottom has only one peak around $x=0.6$ (as labeled by ' $A$ ' in Figure $8-31 \mathrm{~b}$ ), at which a flow injection is induced by vortex I and the corner vortex III. Note the vortex III is stable compared with the corner cortex II, whose shape various dramatically (Figure 8-31). The Nusselt number near the right wall mildly varies, compared to that near the left wall (Figure 8-30b).
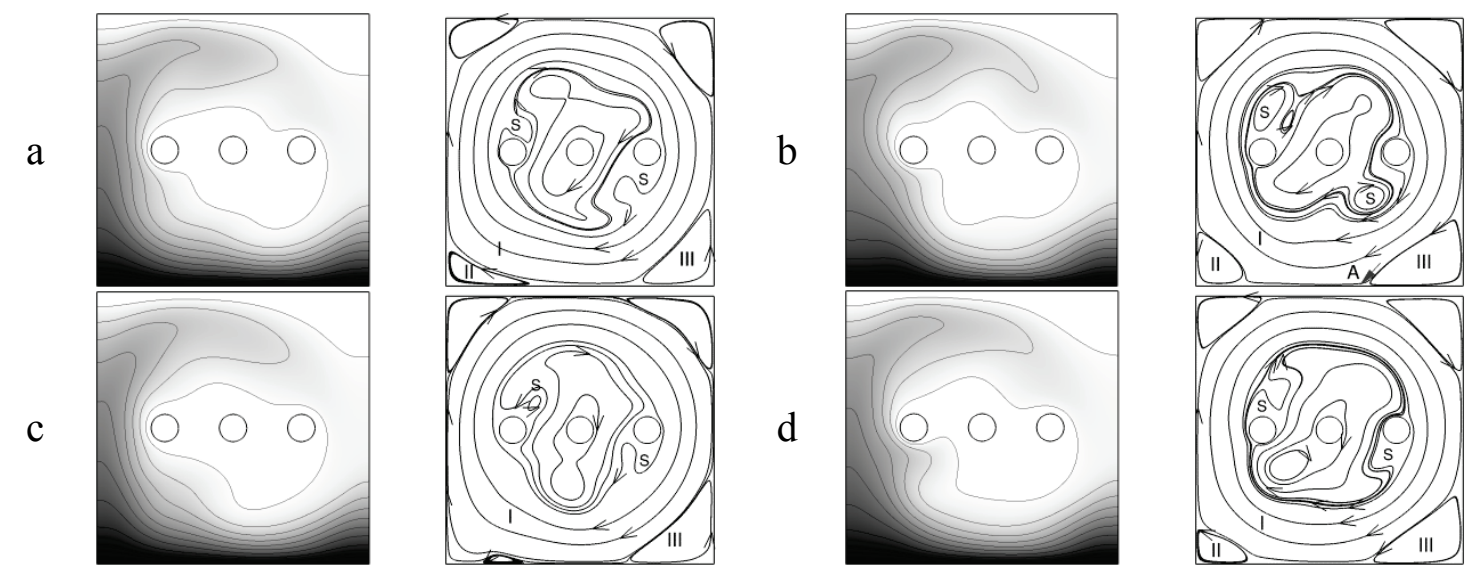

Figure 8-31. $N u$ and Isotherm at $P r=0.021$ and $R a=10^{5}$

(The transient state seems interesting.)

\subsubsection{3 $P r=0.021, R a=10^{6}$}

For the case of the empty enclosure with $P r=0.021$ and $R a=10^{6}$, the surface-averaged Nusselt number is shown in Figure 8-32. The flow structure shown in Figure 8-33 starts with a four-cell structure, and eventually develops into a unicellular structure. However, the center of the enclosure is characterized by a pair of vortices rotating in a clockwise direction. Since the two vortices rotate in the same direction, they chase each other around the center of the enclosure. Figure 8-33 b-f show approximately a half cycle of the vortex structure development in the enclosure. 


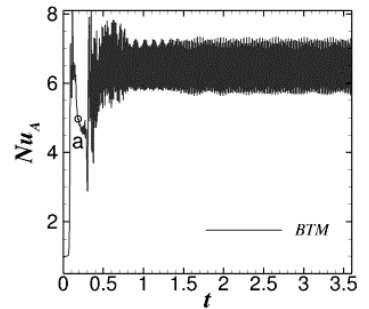

(a) $N u_{A}$

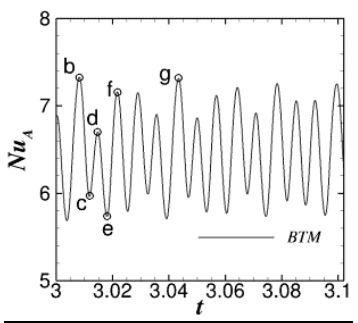

(b)

Figure 8-32: $N u_{A}$ and Isotherm at $P r=0.021$ and $R a=10^{6}$ for the Reference Case

a

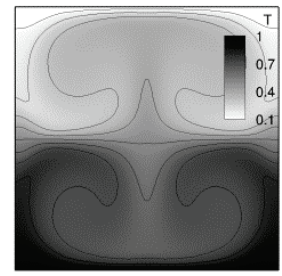

c

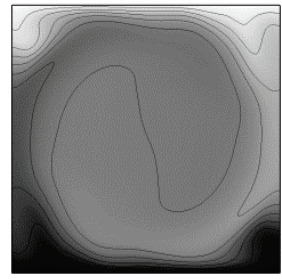

e

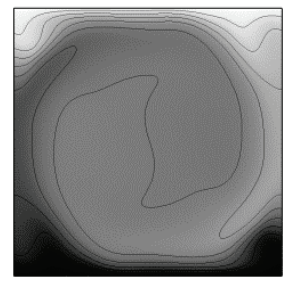

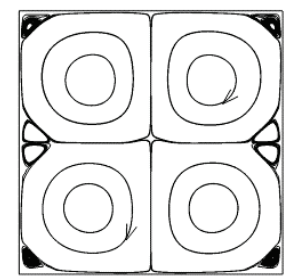
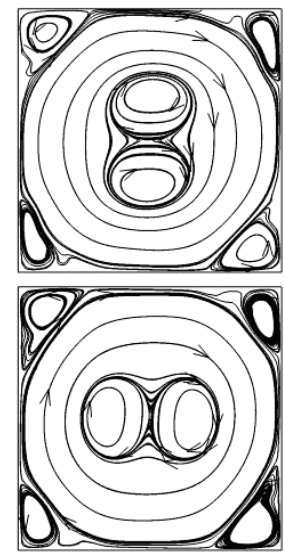

$\mathrm{b}$

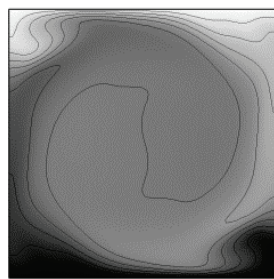

d
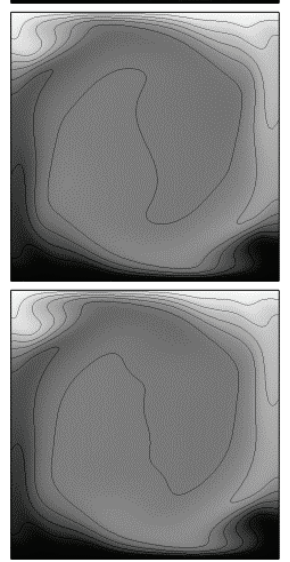
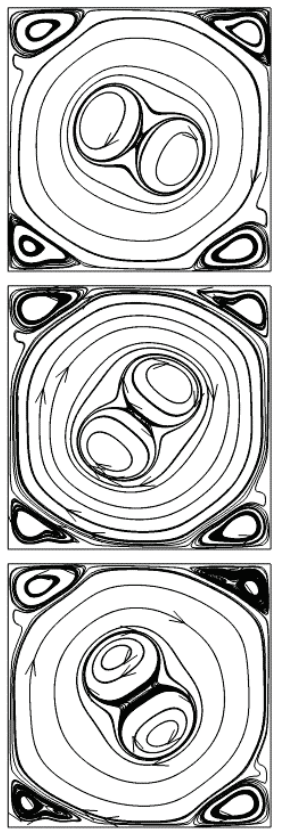

Figure 8-33. Isotherms and Streamlines of the Reference Case at $P r=0.021$ and $R a=10^{6}$

As one cylinder is inserted, a four-cell structure is initially formed (the first stage, Figure 8-34 b), then the diagonal vortices are merged (Figure 8-34d) and the flow is dominated by a unicellular circulation (the second stage, Figure 8-34c). The time-averaged isotherms and streamlines are presented in Figure 8-34 (e) and (f), respectively. 


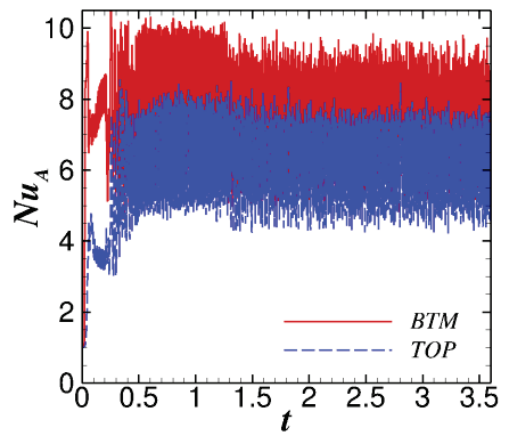

(a)

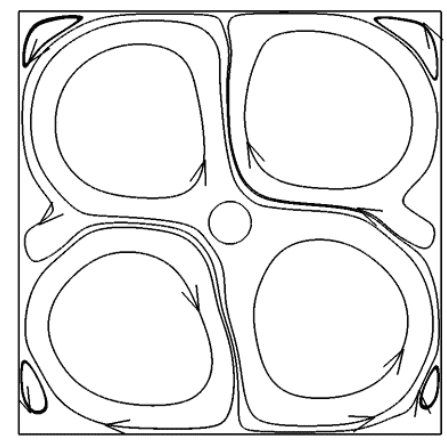

(d)

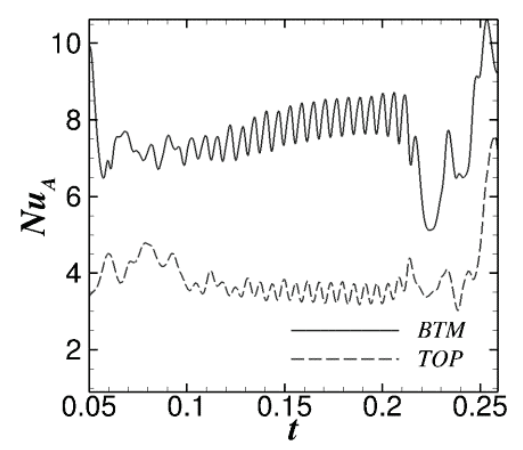

(b)

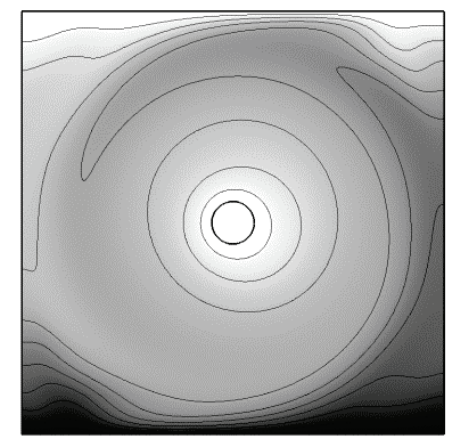

(e)

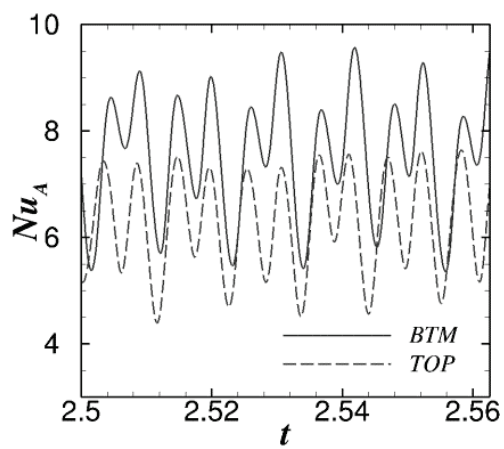

(c)

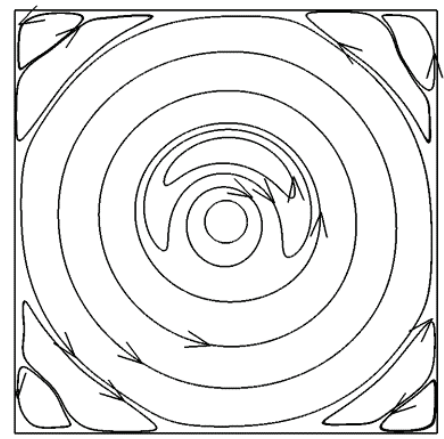

(f)

Figure 8-34: $\mathbf{N u _ { A }}$ at the Bottom and Top Surfaces

$$
\text { ( } P r=0.021 \text { and } R a=10^{6} \text {, with one cylinder in the center.) }
$$

As two cylinders inserted in the enclosure, the surface-averaged Nusselt number shows periodic oscillation (Figure 8-35). The time-averaged isotherms and streamlines are presented in Figure 8-35 (c) and (d). 


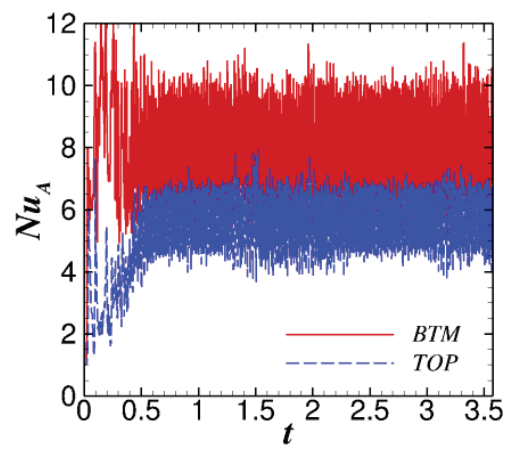

(a)

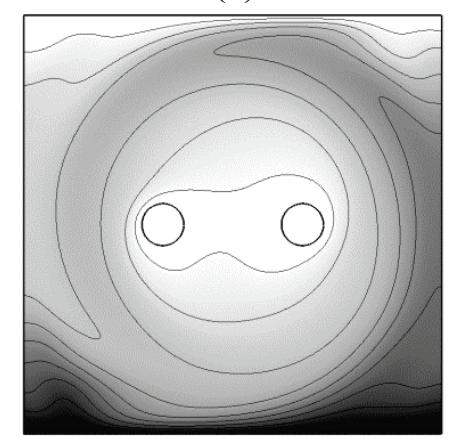

(c)

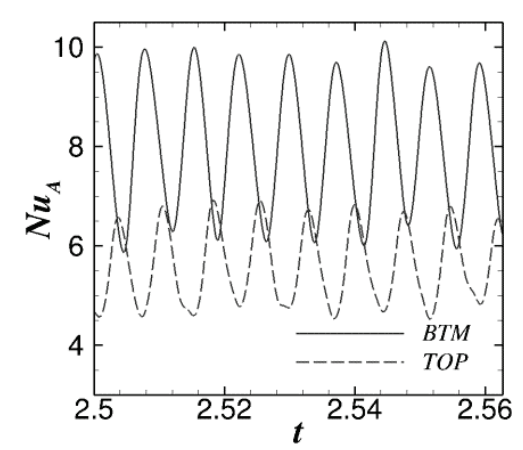

(b)

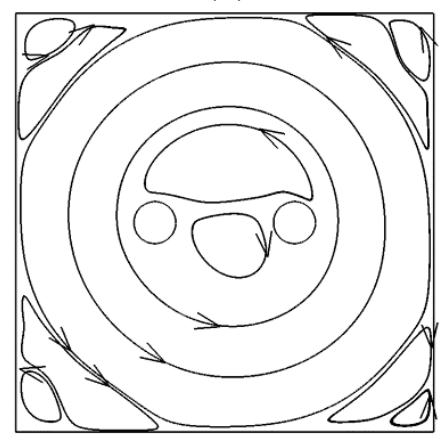

(d)

Figure 8-35: Surface-Averaged Nusselt Number Showing Periodic Oscillation

$\left(\mathrm{Pr}=0.021\right.$ and $\mathrm{Ra}=10^{6}$, with two cylinders in the center. (a) $\mathrm{Nu}$ at the bottom and top surfaces; (b) Periodic solution; (c) time averaged isotherms; (d) Time averaged streamlines.)

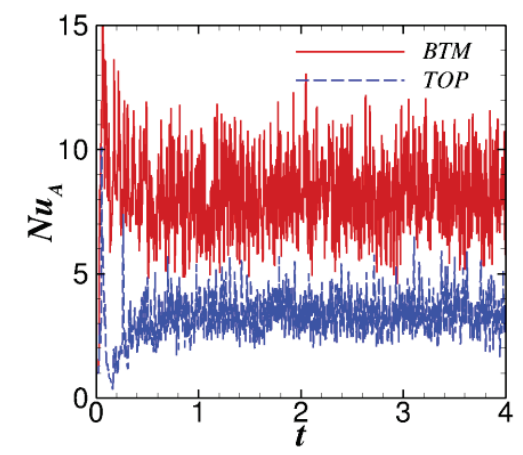

(a)

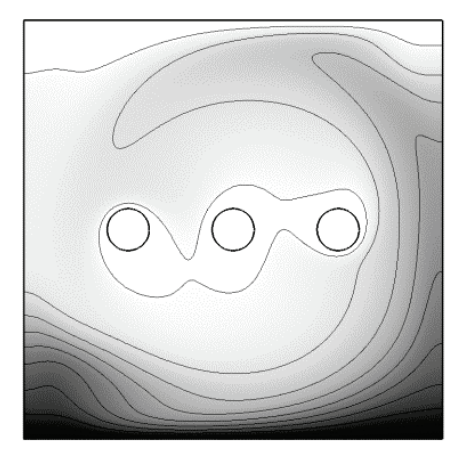

(b)

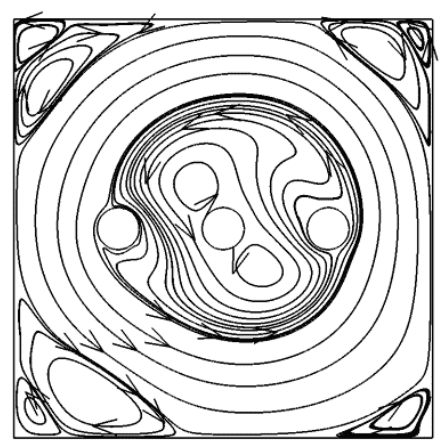

(c)

Figure 8-36: Time-averaged Isotherms and Streamlines

$\left(\mathbf{N u _ { A }}\right.$ at the bottom and top surfaces. $\mathrm{Pr}=0.021$ and $\mathrm{Ra}=10^{6}$, with three cylinders in the center. (a) $\mathbf{N} \boldsymbol{u}_{\boldsymbol{A}}$ at the bottom and top surfaces. (b) time averaged isotherms (d) time averaged streamlines.)

As three cylinders are inserted in the enclosure, the heat transfer on the bottom surface is further enhanced, as shown in the Figure 8-36.

Table 8-4 summarized the surface-averaged Nusselt number at the hot surface for the cases of $\operatorname{Pr}=0.021$. At the low Rayleigh number $10^{4}$, heat transfer in an empty enclosure is dominated by the conduction. Inserting cold cylinders, however, can induce the initiation of heat convection and therefore enhance the heat transfer at the hot bottom. At $R a=10^{5}$ and $R a=10^{6}$, inserting cylinders can induce more vigorous convection.

DISTRIBUTION STATEMENT A: Approved for public release. Distribution is unlimited. 
Table 8-4: Nusselt Number at $\operatorname{Pr}=\mathbf{0 . 0 2 1}$, Various $R a$, and Number of Cylinders

\begin{tabular}{|l|l|l|l|l|}
\hline$R a$ & $10^{3}$ & $10^{4}$ & $10^{5}$ & $10^{6}$ \\
\hline Ref. & 0.991 & 1.008 & $3.844 \pm 0.162$ & $6.465 \pm 0.408$ \\
\hline 1 Cyl. & 1.574 & 2.012 & $4.611 \pm 0.363$ & $7.668 \pm 0.946$ \\
\hline 2 Cyl. & 1.877 & 3.311 & 7.257 & $8.056 \pm 1.174$ \\
\hline 3 Cyls. & 1.992 & 2.210 & $4.901 \pm 0.108$ & $8.509 \pm 1.179$ \\
\hline
\end{tabular}

\subsection{Conclusions}

In this study, we investigated the natural convection in a vertical enclosure with and without inserted small circular cylinder(s). The ranges of the Rayleigh number and the Prandtl number are $10^{3}-10^{6}$, and $0.021-7.1$, respectively.

At low Rayleigh number such as $10^{3}$, at which the heat transfer in the enclosure is in the heat conduction regime, inserting cold cylinders along the horizontal center line can enhance the heat transfer at the bottom surface by the increased thermal gradient. Three uniformly placed cylinders can basically double the surface-averaged Nusselt number at the hot bottom.

At Rayleigh number $10^{4}$, the heat transfer mechanism and its variation after cylinders being inserted depend on the Prandtl number. Especially, heat convection can be induced in the enclosure filled with low Prandtl number $(P r=0.021)$ fluids by cold cylinders, as opposed to the heat conduction occurred in the counterpart empty enclosure.

When $R a$ is increased to $10^{5}$, at which head convection dominates in the enclosure. Inserting two or three cylinders in the enclosure can comparably enhance the heat transfer at the bottom surface, for all the three Prandtl numbers we studied. Placing more cylinders along the horizontal centerline can actually diminish the heat convection in the enclosure.

As $R a=10^{6}$, flow oscillation are obtained for all the three Prandtl numbers studied here. However, the flow structures are totally different among the three fluids. As cold cylinders are inserted into the enclosure, the heat transfer is enhanced through the induced flow disturbance. At this Rayleigh number, five or seven cylinders may be needed to get the most enhancements in terms of the surface-averaged Nusselt number at the hot bottom. 


\section{THERMAL EFFECTS ON VORTEX-INDUCED VIBRATIONS}

\subsection{Introduction}

Vortex-induced vibration (VIV) of structures is of practical interest to many engineering fields. The suppression of VIV can be achieved passively or actively. In the category of passive control of VIV, methods such as surface protrusion (strakes, wires etc.), shrouds (e.g., axial rods), and near wake stabilizers (splitters, slits cuts etc.) were discussed [259, 260]. For example, Owen et al. [261] studied passive control of VIV and drag reduction of cylinders with hemispherical bumps. Using tripping wires near a cylinder as near wake stabilizers, Hover et al. [262] found that the wire introduces significant changes, as functions of the reduced frequency. Strykowski et al. [263] found that the vortex shedding behind circular cylinders can be suppressed by properly placed small control cylinder in the near wake of the main cylinder. Vortex shedding is associated with temporally unstable eigenmodes which are heavily weighted by the near field [263]. Kwon et al. [264] used splitter plates to control the laminar vortex shedding behind a circular cylinder. They found that the vortex shedding completely disappeared when the length of the splitter plate is larger than a critical length. As one of the most widely used devices to suppress VIV, the helical strake, has the disadvantage that it increases drag. In the category of active control of VIV, suppression of VIV was studied using blowing/suction-based flow control [265, 266]. Ffowcs Williams et al. [267] proposed a method to actively control vortex shedding using acoustic feedback system, and this method was further investigated by Hiejima et al. [268, 269].

We propose a method to suppress the vortex-induced vibration of a cylinder by varying its temperature [270,271]. The flow direction and the thermal induced buoyancy force are aligned to fully take advantage of the thermal effect. When the flow past a cylinder with elevated temperature, essentially mixed-convection occurs, in which both natural and forced convection are involved. The relative importance of the natural convection and the forced convection is characterized by the Richardson number. The flow separation around the cylinder and suppression of VIV at various Richardson number are numerically studied for structures with different stiffness.

\subsection{Mathematical Formulations}

With incompressible flow and Boussinesq approximation, the Navier-Stokes equation and thermal equation have the form of:

$$
\begin{gathered}
\nabla \cdot \mathbf{u}^{*}=0 \\
\frac{\partial \mathbf{u}^{*}}{\partial t^{*}}+\mathbf{u}^{*} \cdot \nabla \mathbf{u}^{*}=-\frac{1}{\rho_{0}} \nabla p^{*}+\nu \nabla^{2} \mathbf{u}^{*}+\mathbf{g} \beta\left(\Delta \theta^{*}\right) \\
\frac{\partial \theta^{*}}{\partial t^{*}}+\mathbf{u}^{*} \cdot \nabla \theta^{*}=\kappa \nabla^{2} \theta^{*}
\end{gathered}
$$

in which the primitive variables $\mathbf{u}^{*}, p^{*}$ are dimensional velocity and pressure respectively, and $\theta^{*}$ is the dimensional temperature. The thermal expansion coefficient, thermal diffusivity, kinematic viscosity, and the density of the fluid are denoted by $\beta, \kappa, v$, and $\rho_{0}$ respectively.

Taken the diameter of the cylinder $D$ as the characteristic length, the free stream velocity $U$ as the characteristic velocity, the non-dimensional Navier-Stokes equation and thermal equation have the form of:

$$
\nabla \cdot \mathbf{u}=0
$$




$$
\begin{gathered}
\frac{\partial \mathbf{u}}{\partial t}+\mathbf{u} \cdot \nabla \mathbf{u}=-\nabla p+\frac{1}{R e} \nabla^{2} \mathbf{u}+R i \theta \\
\frac{\partial \theta}{\partial t}+\mathbf{u} \cdot \nabla \theta=\frac{1}{R e P r} \nabla^{2} \theta
\end{gathered}
$$

in which the Reynolds number $\operatorname{Re}(=U D / v)$, is the ratio of the inertial force to the viscous force. The Prandtl number $\operatorname{Pr}(=v / \kappa)$, the ratio of the kinematic to the thermal diffusivity, is a property depending on the fluid medium only. In this study, the fluid medium is water, and its Prandtl number is 7.1. The Richardson number $R i\left(=g \beta \Delta \theta D / U^{2}\right)$ characterizes the buoyancy force and the inertial force. When the heat transfer between the cylinder and the fluid medium is dominated by the forced convection, the Richardson number is far below the unity, i.e., $R i \ll 1$. On the other end, when the natural convection dominates heat transfer, $R i \gg 1$. The term $R e P r$ in equation (9$6)$ can also be written as the Peclet number $P e(=R e P r)$. The velocity boundary condition on the cylinder surface in our study are non-slip and impermeable, i.e., $\mathbf{u}=0$. The temperature of the cylinder and the upcoming flow are set to 1 and 0 , respectively.

The general equation of motion of an elastically mounted cylinder is as follows:

$$
m \frac{\partial^{2} Q_{i}}{\partial t^{2}}+c \frac{\partial Q_{i}}{\partial t}+k Q_{i}=F_{i}
$$

$Q_{i}$ is the i-th component of the position (displacement) vector of the cylinder center, and $c$ is the dimensional damping coefficient.

The non-dimensional equation of body motion is then:

$$
\frac{\partial^{2} Q_{i}}{\partial t^{2}}+\frac{4 \pi \zeta}{U_{r e d}} \frac{\partial Q_{i}}{\partial t}+\frac{4 \pi^{2}}{U_{r e d}^{2}} Q_{i}=\frac{1}{2 M_{r e d}} C_{i}
$$

in which the reduced velocity $U_{\text {red }}$ is defined as $U_{\text {red }}=\frac{U}{f D}$, and the reduced mass $M_{\text {red }}=\frac{m}{\rho_{f} D^{2}}$. The natural frequency of the cylinder is denoted by $f$, which is defined as $f=\frac{1}{2 \pi} \sqrt{k / m}$. The subscript ' $i$ ' stands for the motion direction. The structure is getting more flexible, and the natural frequency of the structural vibration is smaller, when the reduced velocity increases. $C_{i}$ in the body motion equation is the hydrodynamic force coefficient. The damping coefficient $c$ or $\zeta$ is set to be zero in the current study. The Navier-Stokes equations and the body motion equation are coupled in a similar way as that in $[17,31]$.

\subsection{Numerical Method and Validation}

\subsubsection{Numerical Method}

The Navier-Stokes equations are discretized using a cell-centered, collocated arrangement of the primitive variables $(\mathbf{u}, p)$ and integrated in time using fractional step method [16]. Secondorder central difference schemes in space are employed to both convection and diffusion terms in momentum and temperature equations.

\subsubsection{Validaton}

Validations were conducted against cases in [272], in which flow past an elastically mounted cylinder with reduced mass $M_{\text {red }}=2$ under various reduced velocities $U_{\text {red }}$ (related to 
structural stiffness). The Reynolds number used in the simulation is 150. Figure 9-1 shows the phase changes between the lift coefficient $\left(C_{L}\right)$ and the cross-flow vibration amplitude at two selected structural stiffness. At larger $U_{\text {red }}$ (e.g., 8.0), the vertical-direction vibration and the lift coefficient vary out-of-phase. As the lift coefficient is positive, the displacement of the cylinder is, however, negative, and vice versa. At smaller $U_{\text {red }}$ (e.g., 4.0), the lift coefficient and the vibration in vertical direction vary in phase. The phase plots obtained in the present work are similar to those in the studies of Ahn et al. [272].

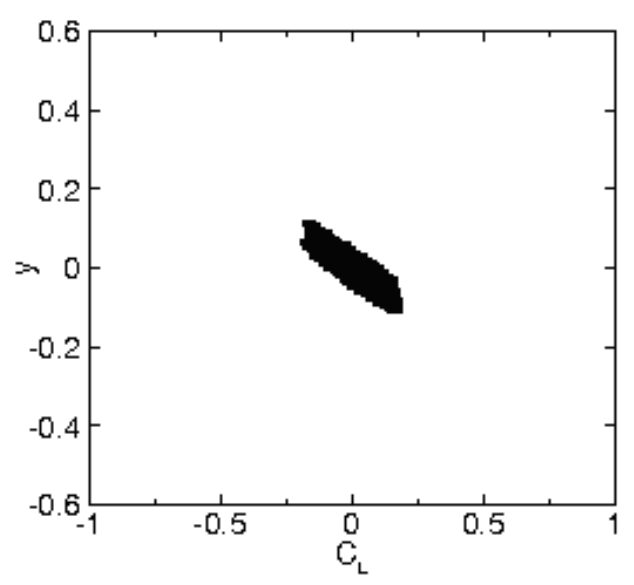

(a) $U_{\text {red }}=8.0$

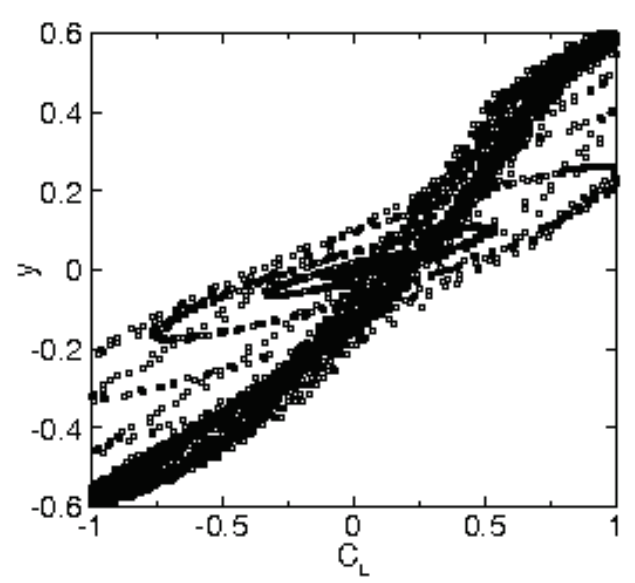

(b) $U_{\text {red }}=4.0$

Figure 9-1: Phase Changes between Lift Coefficient and Vibration Amplitude, $M_{\text {red }}=2$
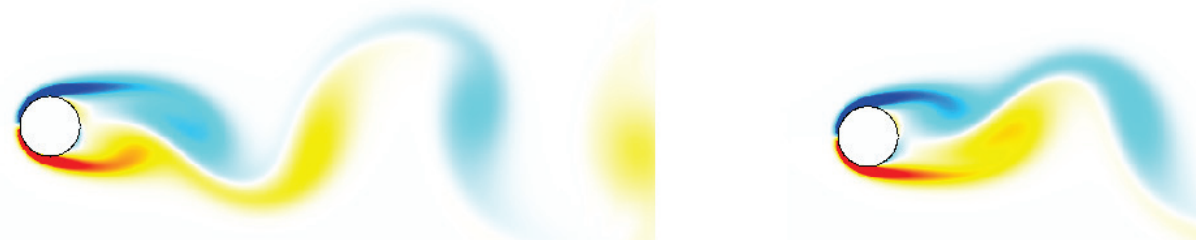

(a) $y_{\max }$

(b) $y_{\min }$

Figure 9-2: Vortex Structure at Extreme Positions (2S mode), $R e=150, M_{\text {red }}=2.0, U_{\text {red }}=$ 8.0 


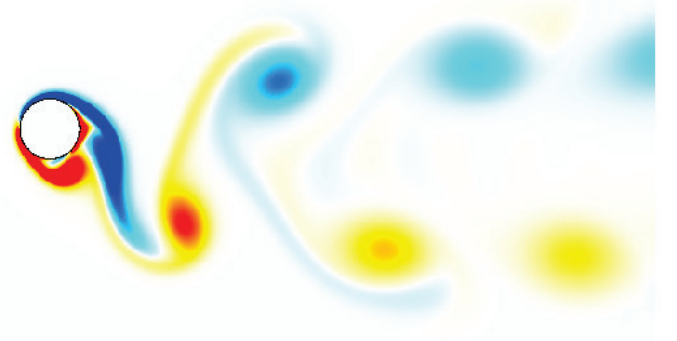

(a) $y_{\max }$

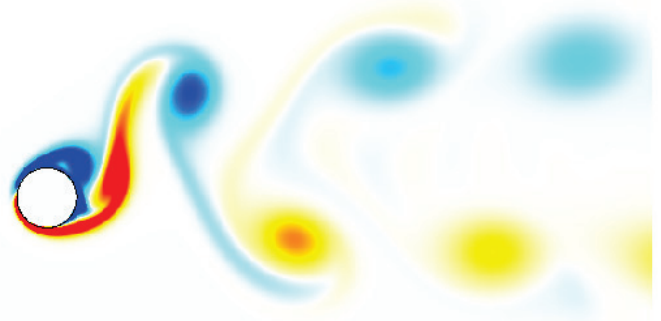

(b) $y_{\min }$

Figure 9-3: Vortex Structure at Extreme Positions, $R e=150, M_{\text {red }}=2.0, U_{\text {red }}=4.0$

The flow structures of the two selected reduced velocities are shown in Figure 9-2 and Figure 9-3, respectively. At $U_{\text {red }}=8.0$, the structural stiffness is lower. The oscillation of the cylinder is small, as shown in Figure 9-1(a). The corresponding flow structure, presented in Figure 9-2 for the cylinder instantaneously located at the uppermost and lowermost positions, is therefore similar to the von Karman vortex street obtained from flow past a fixed cylinder. The wake structure in Figure 9-2 is consisted of single vortices (S) with opposite signs, and is identified as $2 \mathrm{~S}$ mode [273]. At $U_{\text {red }}=4.0$, the oscillation of the cylinder is greater than its lower stiffness counterpart (Figure 9-2). Figure 9-3 shows the vortices are generated and shed in two rows. In the near field right behind the cylinder, a vortex pair $(\mathrm{P})$ is shed in each row. The wake structure is therefore claimed as 2P mode [273], which was experimentally identified by Brika et al. [274]. Note although the vortex pair can be identified in the near wake, it quickly diffuses after 6-8 unit length (the cylinder diameter) in the downstream.

Table 9-1: Comparison on Nusselt Number for a Square Cavity with Lateral Temperature Difference

\begin{tabular}{lllll}
\hline & $R e$ & $M_{\text {red }}$ & $U_{\text {red }}$ & \multicolumn{1}{c}{$y_{\text {max }}$} \\
\hline Ahn & 150 & 2.0 & 4.0 & 0.54 \\
Ahn & 150 & 2.0 & 5.0 & 0.54 \\
Boraziani & 150 & 2.0 & 4.0 & 0.56 \\
Present & 150 & 2.0 & 4.0 & 0.607 \\
Present & 150 & 2.0 & 5.0 & 0.58 \\
\hline
\end{tabular}

\subsection{Results and Discussions}

In this study, we consider a cylinder that can freely oscillate in the x-direction. Its degree of freedom in y-direction, however, is fixed. Flow passes through the cylinder from the bottom to the top, and the cylinder is subject to vibration when vortices are generated and shed periodically. The cylinder is heated, and thermal effects on the vortex-induced vibration will be studied. The Reynolds number is 150 , and the reduced mass $M_{\text {red }}$ is 2.0 throughout this study. 


\subsubsection{Reduced Velocity $U_{\text {red }}=\mathbf{8 . 0}$}

High reduced velocity indicates structures with lower stiffness. In the current study, $U_{r e d}=$ 8.0 and $U_{\text {red }}=4.0$ were selected. The structure with $U_{\text {red }}=8.0$ was presented first.

\subsubsection{1 $\quad U_{\text {red }}=8.0, R i=0$}

Pure forced convection $(R i=0)$ occurred when flow passing a heated cylinder was investigated as the reference case. The cylinder is fixed in y-direction, but can freely move back and forth in the x-direction. Figure 9-4 shows the time course of the oscillation in the transient and quasi-periodic states. The amplitude of the oscillation is smaller than 0.1 (i.e., $10 \%$ of the cylinder diameter). Flow structure at certain time instants are presented in Figure 9-5. At the initial stage, e.g., $t=30$, symmetric vortices were developed (Figure 9-5a), and the cylinder experiences zero net force in the $\mathrm{x}$-direction. Therefore, the cylinder has no motion even though it can potentially move freely. At $t=50$, instabilities develop in the wake, and flow symmetry is broken. Note the wake away from the cylinder shows stronger asymmetry than the wake right above the cylinder.

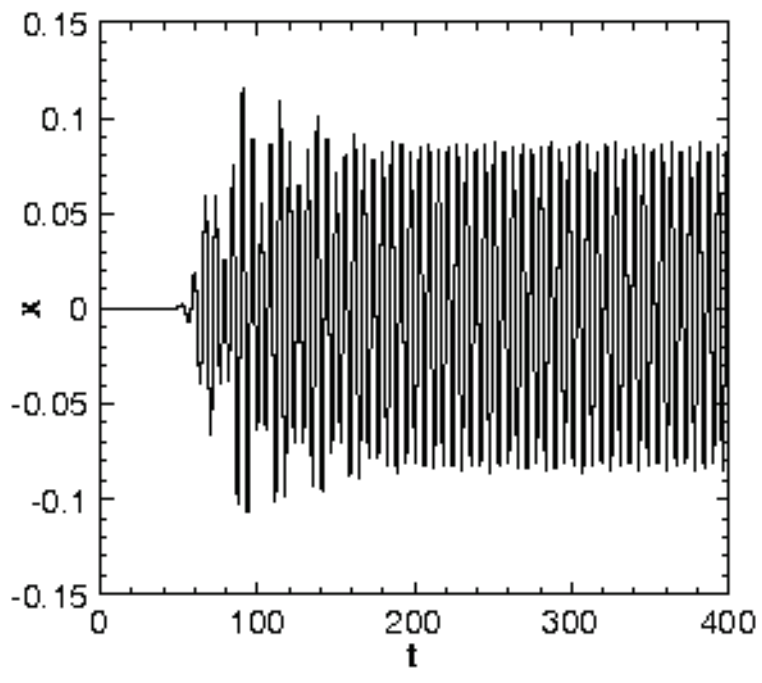

(a) Transient

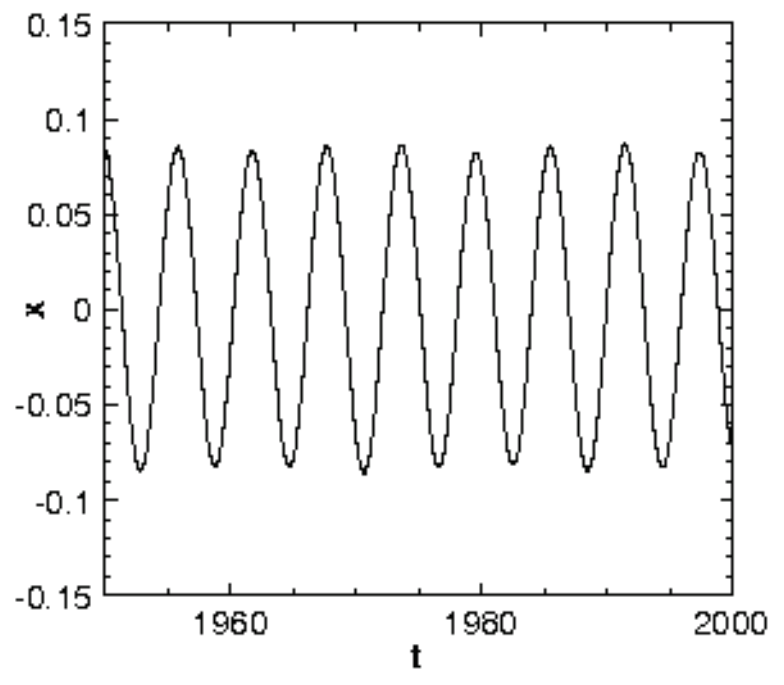

(b) Periodic

Figure 9-4: Time History of the Displacement of Cross-stream Vibration, $R i=0$

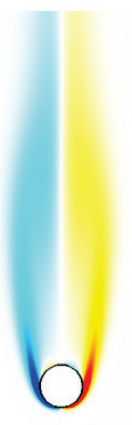

(a) $t=30$

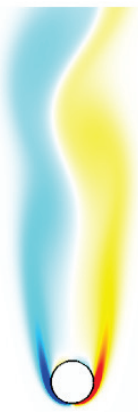

(b) $t=50$

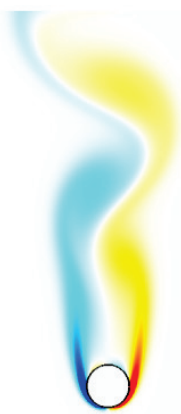

(c) $t=60$

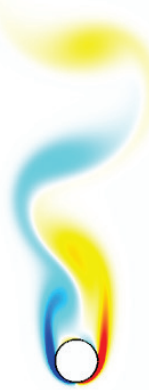

(d) $t=1950$

Figure 9-5: Vortex Development at Certain Time Instants, $U_{\text {red }}=8.0, R i=0$ 


\subsubsection{2 $\quad U_{\text {red }}=8.0, R i=0.1,0.2$, and 0.25}

As small Richardson numbers (e.g., $0.1 \sim 0.25$ ) are applied, i.e., the effects of natural convection increases gradually, the flow structures are close to the one obtained from the case of pure forced convection $(R i=0)$. As shown in Figure 9-6, vortices still shed from the cylinder, the width of the wake, however, get narrower when $R i$ increases. The displacement of the vibration is shown in Figure 9-7 in which the amplitude of the oscillation is gradually reduced when $R i$ increases. As the Richardson number increases to 0.25 , the magnitude of VIV is less than $4 \%$ of the cylinder diameter.

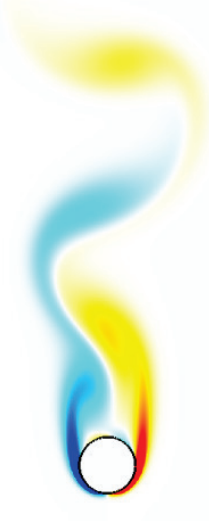

(a) $R i=0.1$

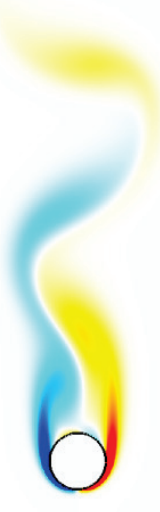

(b) $R i=0.2$

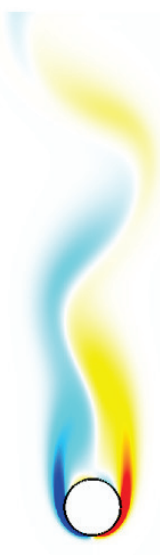

(c) $R i=0.25$

Figure 9-6: Vortex Structure at Extreme Position, for Various $R i$ numbers, $U_{\text {red }}=\mathbf{8 . 0}$

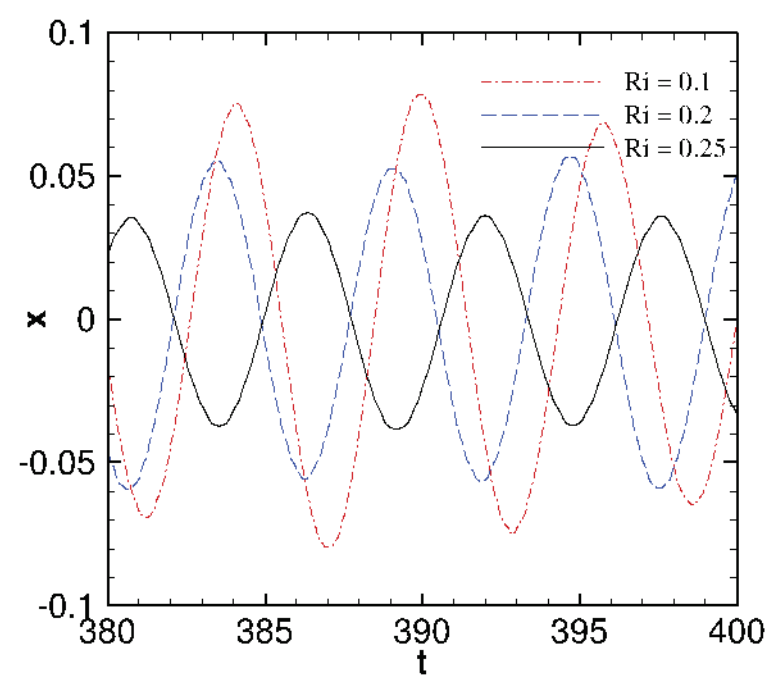

Figure 9-7: Time History of the Displacement

\subsubsection{3 $\quad U_{\text {red }}=8.0, R i=0.3$, and 0.5}

When the Richardson number is increased to 0.3 , there are no vortices formed and shed behind (above) the cylinder. The wake behind the cylinder wiggles around the vertical centerline, but not enough to induce strong vibration of the cylinder. When $R i$ is 0.3 , the cylinder vibration amplitude is reduced to $0.8 \%$ on the cylinder diameter (Figure 9-8b). As $R i$ increased to 0.5 , the amplitude of VIV is around $0.3 \%$ of the cylinder diameter (not shown here). A typical flow 
structure is presented in Figure 9-8(c) at $t=500$, a recirculation region is formed behind the cylinder. There exists a critical Richardson number between 0.25 and 0.3 , above which the amplitude of the VIV is dramatically reduced from $4 \%$ to $0.8 \%$ of the cylinder diameter.

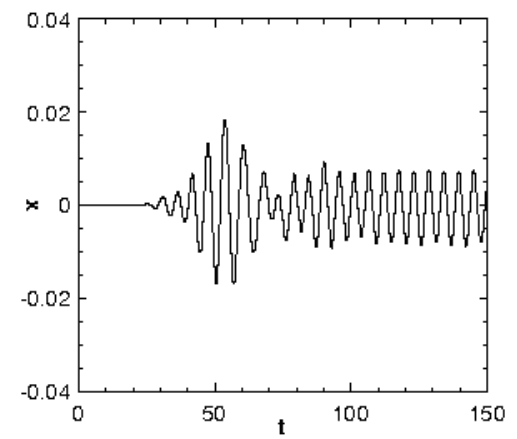

(a) Transient

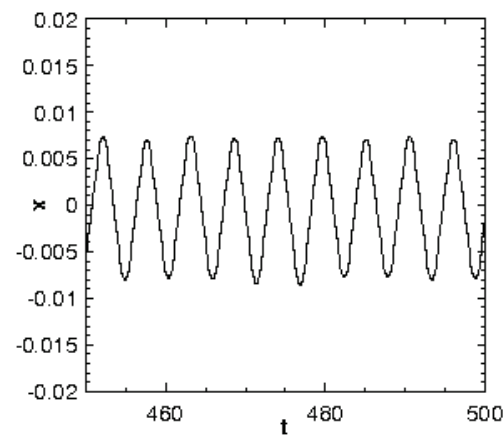

(b) Periodic

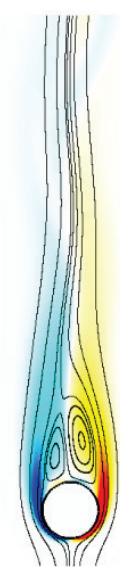

(c) Vortex structure at $\mathrm{t}=500$

Figure 9-8: VIV Displacement and Vortex Structure, $U_{\text {red }}=8.0, R i=0.3$

\subsubsection{Reduced velocity $U_{\text {red }}=\mathbf{4 . 0}$}

In this session, we study the structure with the reduced velocity $U_{\text {red }}=4.0$. The Reynolds number is kept as 150 , and the reduced mass $M_{\text {red }}$ is 2.0 .

\subsubsection{1 $\quad U_{\text {red }}=4.0, R i=0$}

Again, we consider pure forced convection first, i.e., $R i=0$. This simulation is essentially same as the validation case. Figure 9-9 shows the oscillation history at the transient and the periodic states. The oscillation initiated at the non-dimensional time $t \sim 50$. It then reached steady periodic oscillation after $t \sim 90$. The vortex structure in Figure 9-10 is similar to those in the validation case, except with a $90^{\circ}$ turn-around. The $2 \mathrm{P}$ flow structure was found right after the cylinder. 


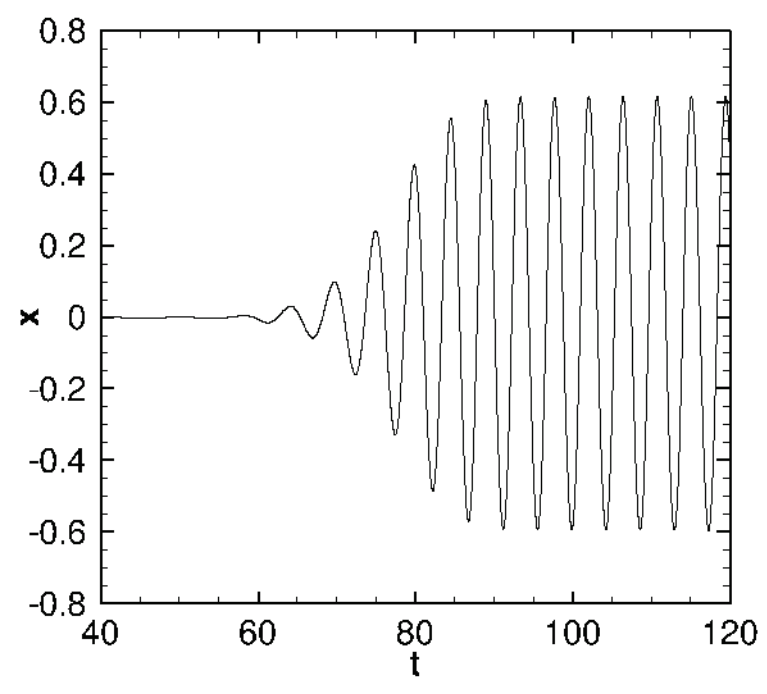

(a) Transient

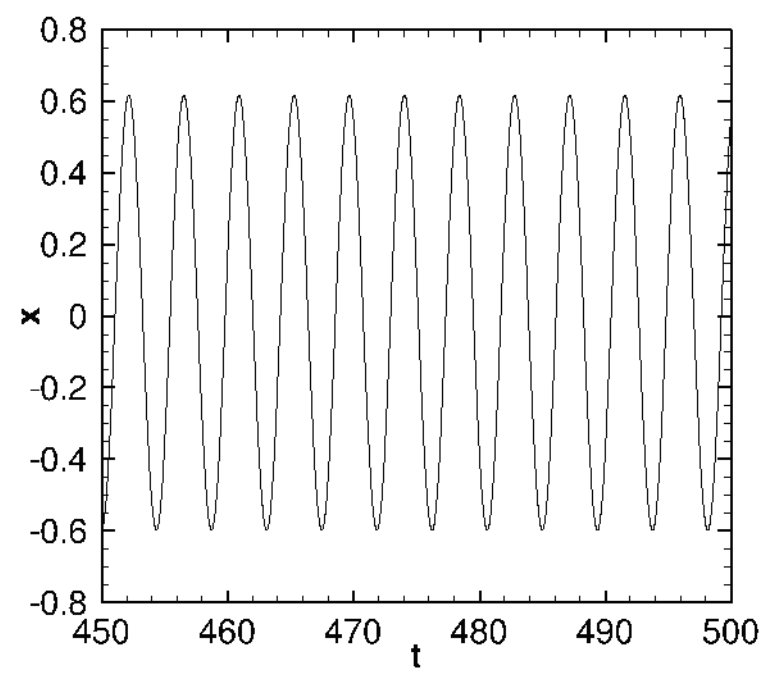

(b) Steady phase

Figure 9-9: Time History of the Displacement of Cross-stream Vibration, $U_{\text {red }}=4.0$, $\boldsymbol{R i}=\mathbf{0}$

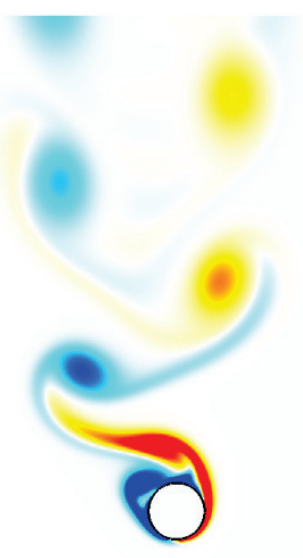

(a) Rightmost

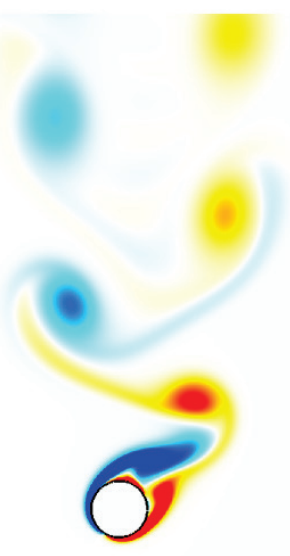

(b) Middle

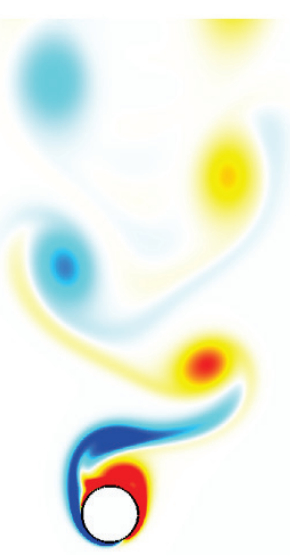

(c) Leftmost

Figure 9-10: Vortex Structure after a VIV Cylinder at Various Locations, $U_{\text {red }}=4.0$, $\boldsymbol{R i}=\mathbf{0}$

9.4.2.2 $\quad U_{\text {red }}=4.0, R i=0.3$

Based on the experience obtained from the structure with lower stiffness, here we started with $R i=0.3$. The displacement in the $\mathrm{x}$-direction is shown in Figure 9-11. It takes shorter time to initiate the vortex induced vibration, compared to the case of $R i=0$ in the previous section. The oscillation amplitude is reduced by $13.2 \%$, as opposed to the case of pure forced convection (Figure 9-10). The vortex structure is shown in Figure 9-12, in which the previous two-column structure (Figure 9-10) is essentially reduced to the one-column structure (Figure 9-12). 


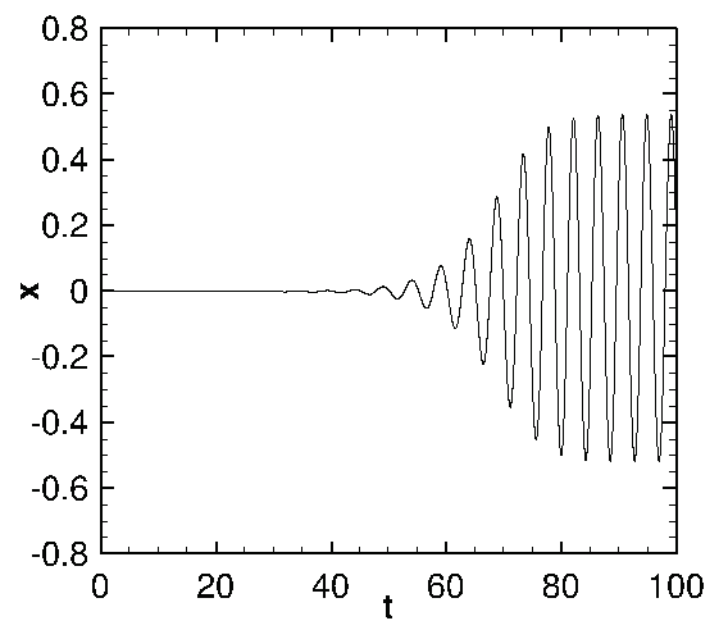

(a) Transient

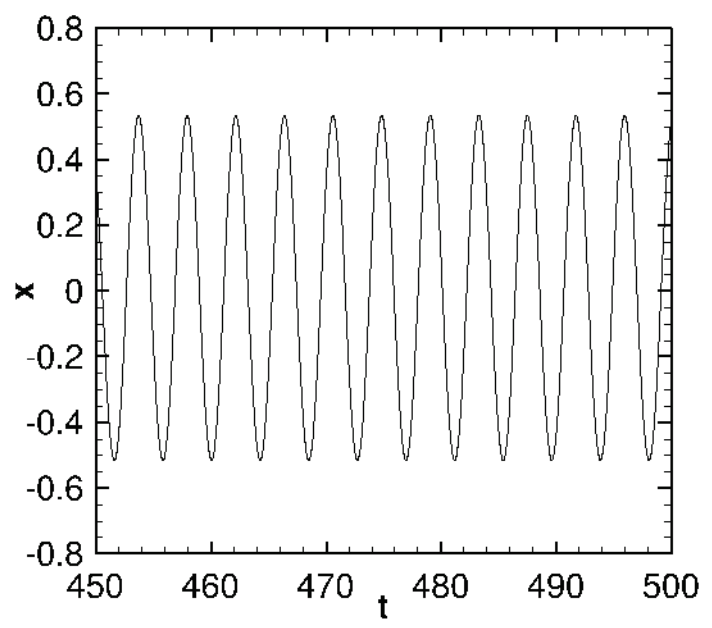

(b) Steady phase

Figure 9-11: Time History of Displacement of Cross-stream Vibration, $U_{\text {red }}=4.0, R i=0.3$

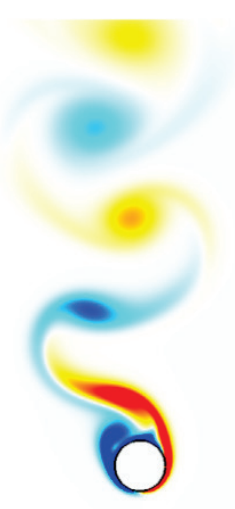

(a) Rightmost

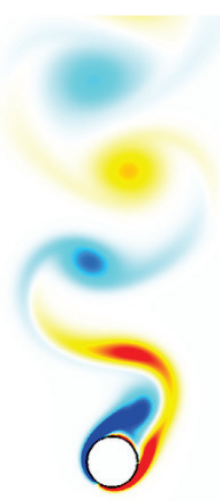

(b) Middle

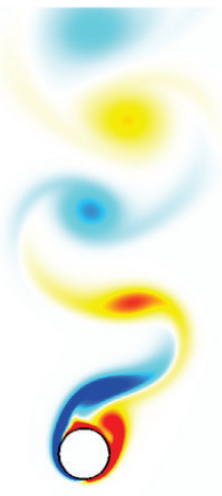

(c) Leftmost

Figure 9-12: Vortex Structure at Various Locations, $U_{\text {red }}=4.0, R i=0.3$

\subsubsection{3 $\quad U_{\text {red }}=4.0, R i=0.5,0.7$ and 0.8}

The displacement of VIV at $R i=0.5,0.7$, and 0.8 was shown in Figure 9-13. As the Richardson number increases, the initiation of the VIV is delayed, and the magnitude of the vibration is also reduced. At $R i=0.5$, the amplitude is around 0.5, i.e., one half of the cylinder diameter. At $R i=0.7$, the vibration magnitude is approximately reduced to 0.42 . The development of flow structure and the temperature contour are shown in Figure 9-14 and Figure 9-15, respectively, for the case of $R i=0.7$. At initial stages such as $t=2$, and $t=5$, typical symmetric vortices were developed behind (above) the cylinder. At $t=10$ and 15, the two typical vortices right behind the cylinder were stretched. Further behind these two typical vortices, the vorticity near the centerline of the cylinder changed the sign. At $t=150$, instability of the vortices near the centerline can be seen. The fully developed $2 \mathrm{~S}$ vortex structure is presented in Figure 9-14 (f). 


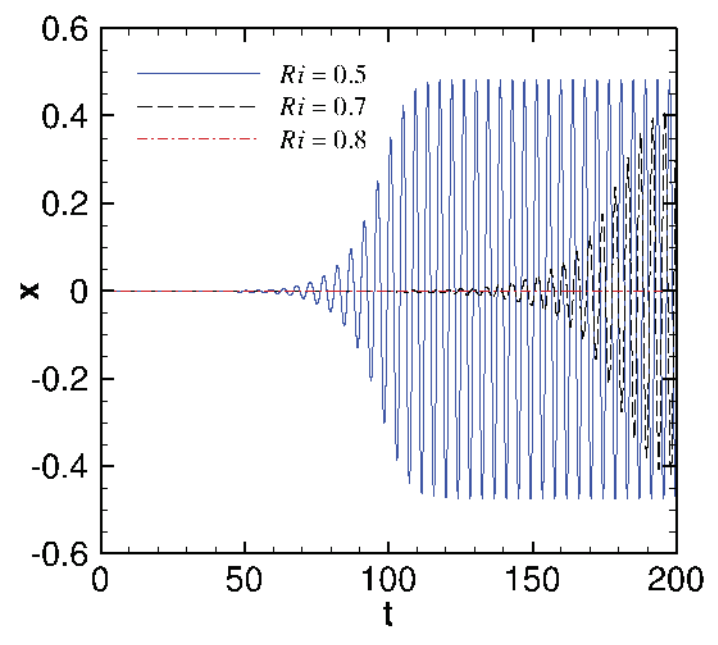

(a) Transient

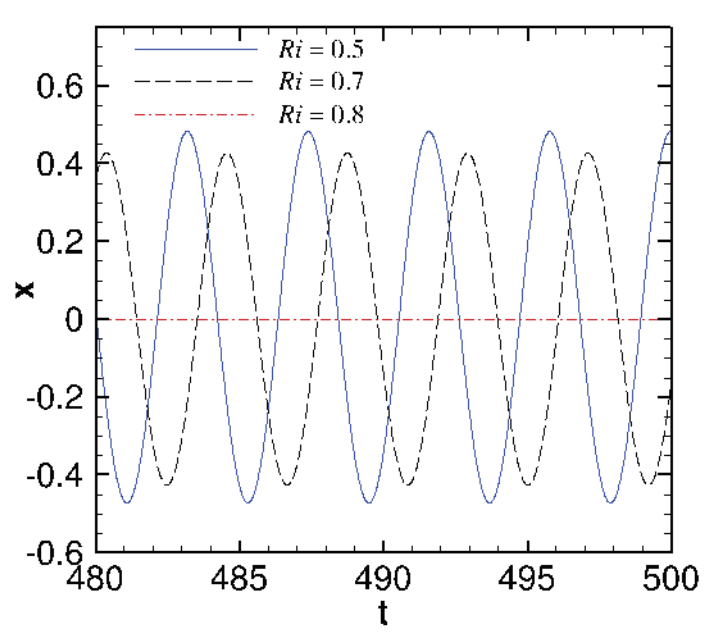

(b) Steady phase

Figure 9-13: Time History of Displacement of Cross-stream Vibration, $U_{\text {red }}=4.0, R i=$ $0.5,0.7$ and 0.8 


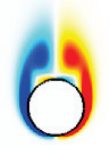

(a) $t=2$

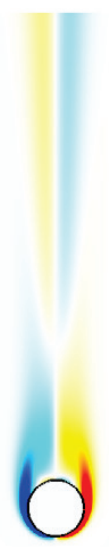

(d) $t=15$

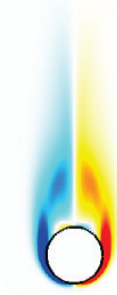

(b) $t=5$

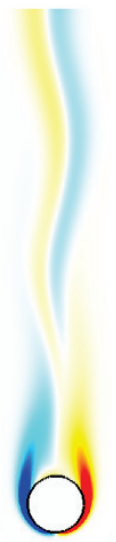

(e) $t=150$

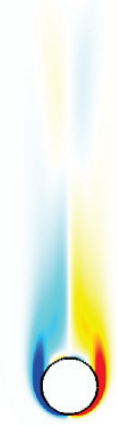

(c) $t=10$

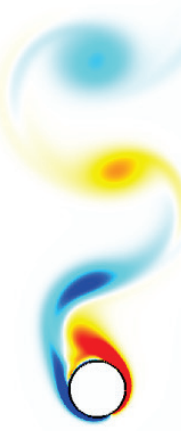

(f) $t=500$

Figure 9-14: Development of Vortex Structure, $U_{\text {red }}=4.0, R i=0.7$

As the Richardson number is further increased to 0.8 , the oscillation is fully suppressed during the simulated time range (Figure 9-13). The vortex structure at $t=500$ is shown in Figure 9-16, in which the flow is symmetric and there is no alternate vortex shedding behind the cylinder. Note the flow structure experienced an abrupt change when $R i$ is increased from 0.7 to 0.8 , and the VIV amplitude is reduced from 0.42 to 0 . There exist a critical Richardson number between 0.7 and 0.8 , above which the flow topology is abruptly changed. The fundamental mechanism needs further investigation.

As compared with previous cases with higher $U_{\text {red }}$ (lower structural stiffness), the suppression of VIV of the structure with lower $U_{\text {red }}$ (higher stiffness) requires higher Richardson number, i.e., stronger natural convection needs to be involved. The critical Richardson number needed to fully suppress the vortex-induced vibration increases with the structural stiffness. 


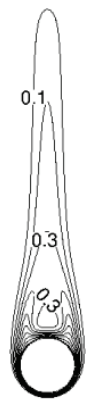

(a) $t=2$

(b) $t=5$

(c) $t=10$
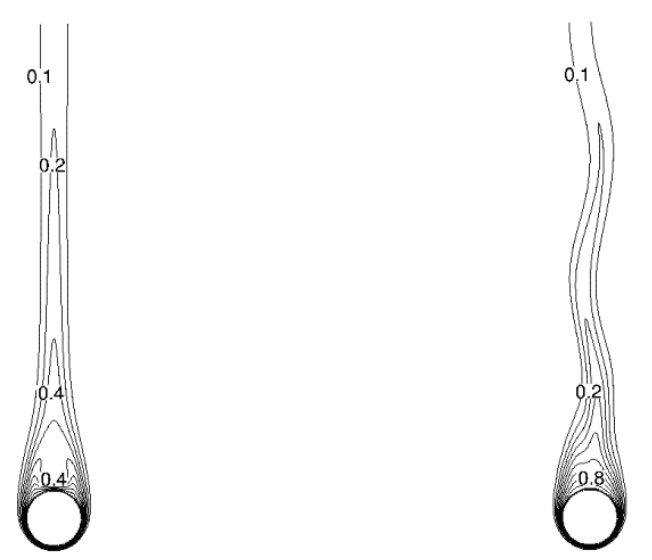

(2)
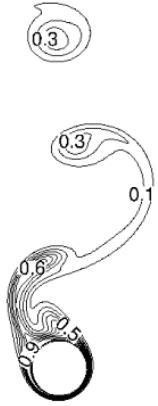

(d) $t=15$

(e) $t=150$

(f) $t=500$

Figure 9-15: Development of Temperature Contour at $U_{\text {red }}=4.0, R i=0.7$ 


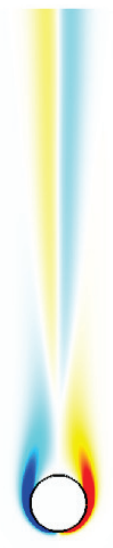

(a) vortex structure

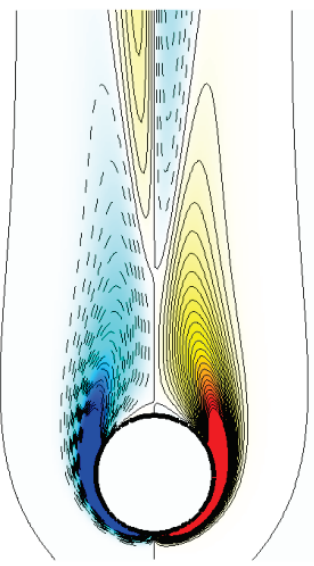

(b) zoom-in on near field

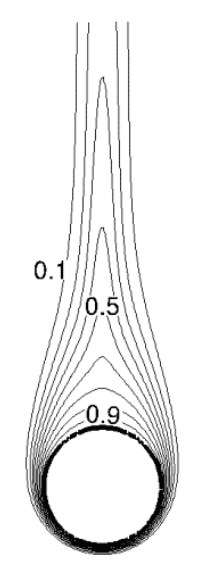

(c) Temperature

Figure 9-16: Flow Past a VIV Cylinder, $U_{\text {red }}=4.0, R i=0.8, t=500$

\subsection{Conclusions}

In this study, the vortex-induced vibration of a cylinder with one degree freedom was numerically simulated. The elastically mounted cylinder is heated and the thermal effect on VIV was investigated. The Reynolds number and the reduced mass were 150 and 2.0, respectively, throughout the current study. Two reduced velocity were selected for the structure, and the simulation was conducted as a mixed-convection problem, in which the relative importance of the natural convection and the forced convection is specified by the Richardson number. It has been shown that the vortex-induced vibration can be reduced when the Richardson number increases, i.e., the natural convection can potentially reduce the VIV when the directions of the flow and the buoyancy force are aligned. Above certain critical Richardson number, the VIV can be fully suppressed. The critical Richardson number depends on the reduced velocity, which is determined by the structural stiffness. For a structural with higher stiffness, the critical Richardson number required to fully suppress the VIV is also higher than that needed for a lower stiffness structure. 


\section{CONCLUSIONS}

We have carried out large-scale molecular dynamics simulations to study the transport properties of liquids and aqueous solutions at liquid-vapor interfaces and in bulk and have demonstrated that molecular dynamics simulations present a powerful tool in investigating the heat transfer behavior of fluid. engineering design and we hope to increase the awareness in the broader community of the usefulness of the computational modeling and molecular dynamics simulations, in particular. We have performed an extensive series of MD simulations computing the transport properties of bulk aqueous surfactant solutions at room and boiling temperatures for a range of sodium dodecyl sulfate surfactant concentrations using flexible and rigid TIP3P water models. It was observed that at higher surfactant concentrations, the rigid water model overestimates the values for surfactant diffusion. At lower concentrations, the results were less reliable due to insufficient statistics. It was recently observed in the case of pure water that the flexible water models are not only more computationally costly but they tend to overestimate the thermal conductivity of neat water. Our results for the thermal conductivity of aqueous SDS solutions show that when the thermal conductivity enhancement of the solution with respect to pure water is concerned, the flexible water model reproduces the experimental trend more reliably, at least at concentrations higher than CMC. Future larger-scale MD studies are needed to further explore and better understand the transport properties of surfactant solutions at low concentrations. We also studied the ability of MP2f, an ab initio water potential derived from condensed phase calculations, to reproduce the diffusion coefficient and thermal conductivity of water at room and boiling temperatures. The bulk water size-independent self-diffusion coefficient was estimated to be $4.05 \times 10^{-5} \mathrm{~cm}^{2} / \mathrm{s}$ at $298 \mathrm{~K}$ and $9.95 \times 10^{-5} \mathrm{~cm}^{2} / \mathrm{s}$ at $373 \mathrm{~K}$. The computed values of the thermal conductivity were: $0.64 \mathrm{~W} /(\mathrm{m} \cdot \mathrm{K})$ at $298 \mathrm{~K}$ and $0.66 \mathrm{~W} /(\mathrm{m} \cdot \mathrm{K})$ at $373 \mathrm{~K}$, in much better agreement with the experimental values compared to both the rigid and the flexible TIP3P.

The liquid volume expansion and diffusion coefficients of the products of the thermal decomposition of ammonium carbamate, carbon dioxide and ammonia, in water and ethylene glycol at standard conditions and at the elevated temperature of the decomposition reaction were studied by carrying out an extensive set of molecular dynamics simulations. Solvent mixtures of water and ethylene glycol were also considered. We compared the solvent compositions for the purposes of using the mixtures as carrier fluid in a heat exchanger utilizing the endothermic decomposition reaction of ammonium carbamate. The modeling and simulation results show that the addition of hydroxyl groups, i.e., ethylene glycol, to aqueous solutions increases the solubility of carbon dioxide. This study points to the observation that compared to water, ethylene glycol is not as well suited to be a carrier fluid for the thermal decomposition reaction of ammonium carbamate. Addition of water could improve the properties of ethylene glycol as a carrier fluid by decreasing the solubility of the product gases and assisting with their removal. Alternatively, a different solvent or mixture can be a suitable carrier fluid in which either both ammonia and carbon dioxide are less soluble or their solubility decreases rapidly at higher gas mole fractions within the temperature range of the decomposition reaction. This study underlines that molecular dynamics simulations offer a powerful low-cost tool in fast throughput and engineering design that can be useful in a broader context of solvent screening for better solubility and heat transfer control beyond the more specific thermal decomposition reaction of ammonium carbamate.

We have used extensive molecular dynamics simulations to study the condensation probabilities of octane. A statistical estimate of the mass accommodation coefficient of octane at five liquid temperatures: $290 \mathrm{~K}, 300 \mathrm{~K}, 310 \mathrm{~K}, 330 \mathrm{~K}$, and $350 \mathrm{~K}$ was provided. The condensation probability of octane was observed to be consistently 0.99 at room temperature $(290 \mathrm{~K}-310 \mathrm{~K})$ and decreasing at higher temperatures. The two octane molecular models, TraPPE and OPLS-AA, 
provided very close values for the condensation probability even though one was a neutral coarsegrained united atom model and the other one was an all-atom model with partial charges, suggesting that in an estimate of the macroscopic condensation coefficient the use of a coarsegrained model is sufficient and justified by the computational savings. Our results are consistent with available data from other simulations and, for the first time, extend to lower temperatures where the condensation probability approaches unity. This work supports that the accommodation coefficient of octane can be considered equal to 1.00 at and around room temperature. We have found that the accommodation coefficient decreases below the value $\left(\alpha_{c}=1\right)$ above $330 \mathrm{~K}$. For the first time, using atomic-scale molecular dynamics simulations, an estimate of the evaporative flux of octane in vacuum was derived.

We have numerically studied the heat transfer behavior of a phase change material in an open-cell micro-foam using both volume-averaged and direct simulations. Heat transfer process was simulated for a spherical-pore embedded micro-foam, and its top surface was kept isothermal. By examining the volume fraction of melted PCM, average temperature at different locations, temperature jump across micro-foam and PCM interface, and average heat flux at the top surface, we find that volume-averaged simulations can reasonably predict the heat transfer process in the micro-foam with complex geometry, as long as accurate thermo-physical parameters such as effective thermal conductivity are provided. In the present study, the effective thermal conductivity of the PCM/micro-foam system was obtained from direct numerical simulation of a unit cell. The simulation results supported the Progelhoff model of effective thermal conductivity where the effective thermal conductivity can be expressed as a power law of porosity and a power factor of 1.3 was calculated for the model structure. We found that the heat flux near the enforced isothermal boundary is dominated by the micro-foam due to its high thermal conductivity. The phase change heat transfer becomes gradually more pronounced in locations away from the enforced high temperature boundary. Direct numerical simulations provided insight into the complicated heat transfer behavior of PCM/foam systems and a three-dimensional PCM melting front and temperature distribution was observed to evolve under constant heat flux. The pressure drops and interstitial heat transfer coefficients were calculated and it was determined that for a micro-foam porosity of $\gamma=0.757$, the heat transfer is conduction dominated and the effects of natural convection can be neglected. DNS results were used to extract thermo-physical parameters for use in volume averaged methods and the effective thermal conductivity was found to be a non-linear function of the micro-foam porosity and the thermal conductivities of the foam and the PCM. The temperature profiles and PCM melt fraction were compared from results from DNS and one- and two- temperature volume average simulations. Two-temperature volume averaged simulations with effective thermal conductivity based on the nonlinear Progelhoff model with power factor derived from DNS was found to match the DNS results. Both DNS and two-temperature volumeaveraged simulations show a four-stage temperature profile during heating: pre-melting, melting, waiting, and post-melting. Considering the much lower computational cost, the two-temperature volume-averaged method with suitable effective thermal conductivity can be a viable method to provide reasonable prediction of the heat transfer behavior in the PCM/micro-foam system and PCM phase change.

We investigated the natural convection in a vertical enclosure with and without inserted small circular cylinder(s). The ranges of the Rayleigh number and the Prandtl number are $10^{3}-10^{6}$, and 0.021-7.1, respectively. At low Rayleigh number such as $10^{3}$, at which the heat transfer in the enclosure is in the heat conduction regime, inserting cold cylinders along the horizontal center line can enhance the heat transfer at the bottom surface by the increased thermal gradient. Three

111

DISTRIBUTION STATEMENT A: Approved for public release. Distribution is unlimited. 
uniformly placed cylinders can basically double the surface-averaged Nusselt number at the hot bottom. At Rayleigh number $10^{4}$, the heat transfer mechanism and its variation after cylinders being inserted depend on the Prandtl number. Especially, heat convection can be induced in the enclosure filled with low Prandtl number $(\mathrm{Pr}=0.021)$ fluids by cold cylinders, as opposed to the heat conduction occurred in the counterpart empty enclosure. When $\mathrm{Ra}$ is increased to $10^{5}$, at which head convection dominates in the enclosure. Inserting two or three cylinders in the enclosure can comparably enhance the heat transfer at the bottom surface, for all the three Prandtl numbers we studied. Placing more cylinders along the horizontal centerline can actually diminish the heat convection in the enclosure. As $\mathrm{Ra}=10^{6}$, flow oscillation are obtained for all the three Prandtl numbers studied here. However, the flow structures are totally different among the three fluids. As cold cylinders are inserted into the enclosure, the heat transfer is enhanced through the induced flow disturbance. At this Rayleigh number, five or seven cylinders may be needed to get the most enhancements in terms of the surface-averaged Nusselt number at the hot bottom.

The vortex-induced vibration of a cylinder with one degree of freedom was numerically simulated. The elastically mounted cylinder is heated and the thermal effect on VIV was investigated. The Reynolds number and the reduced mass were 150 and 2.0, respectively, throughout the current study. Two reduced velocity were selected for the structure, and the simulation was conducted as a mixed-convection problem, in which the relative importance of the natural convection and the forced convection is specified by the Richardson number. It has been shown that the vortex-induced vibration can be reduced when the Richardson number increases, i.e., the natural convection can potentially reduce the VIV when the directions of the flow and the buoyancy force are aligned. Above certain critical Richardson number, the VIV can be fully suppressed. The critical Richardson number depends on the reduced velocity, which is determined by the structural stiffness. For a structural with higher stiffness, the critical Richardson number required to fully suppress the VIV is also higher than that needed for a lower stiffness structure. 


\section{RECOMMENDATIONS}

We have shown that atomistic and continuum computational approaches provide unique perspectives in describing complex processes and phenomena at interfaces including heat transfer and multi-phase flow. Multiscale approaches combining atomistic and continuous models will find broader applicability but further development is needed towards a hybrid multiscale methodology for multicomponent flow models and designs. 


\section{REFERENCES}

[1] J.S. Hansen, J.C. Dyre, P.J. Daivis, B.D. Todd, H. Bruus, Physical Review E, 84 (2011) 036311.

[2] N.K. Voulgarakis, J.-W. Chu, J. Chem. Phys., 130 (2009) 134111.

[3] V. Varshney, S.S. Patnaik, A.K. Roy, B.L. Farmer, Macromolecules, 41 (2008) 6837-6842.

[4] S.S. Patnaik, M. Tsige, Modeling and simulation of nanoscale materials, in: S.M.

Mukhopdhyay (Ed.) Nanoscale multifunctional materials: Science and Applications, John Wiley \& Sons, 2011.

[5] N.B. Shenogina, M. Tsige, S.S. Patnaik, S.M. Mukhopadhyay, Macromolecules, 45 (2012) 5307-5315.

[6] N.B. Shenogina, M. Tsige, S.S. Patnaik, S.M. Mukhopadhyay, Polymer, 54 (2013) 33703376.

[7] V. Varshney, S.S. Patnaik, A.K. Roy, B.L. Farmer, Polymer, 50 (2009) 3378-3385.

[8] V. Varshney, S.S. Patnaik, A.K. Roy, G. Froudakis, B.L. Farmer, Acs Nano, 4 (2010) 11531161.

[9] V. Varshney, S.S. Patnaik, A.K. Roy, B.L. Farmer, Journal of Physical Chemistry C, 114 (2010) 16223-16228.

[10] E. Iskrenova, S.S. Patnaik, Journal of Nanotechnology in Engineering and Medicine 43 (2013) 031001.

[11] S. Manoharan, D. Kalaikadal, R.M. Manglik, M.A. Jog, E. Iskrenova, S.S. Patnaik, J. Heat Transfer, 137 (2015) 080912.

[12] E.K. Iskrenova, S.S. Patnaik, International Journal of Heat and Mass Transfer, 100 (2016) 224-230.

[13] E.K. Iskrenova, S.S. Patnaik, In preparation, (2016).

[14] M. Allen, D. Tildesley, Computer simulations of liquids, Oxford Science, Oxford, 1987.

[15] H. Heinz, S.S. Patnaik, Modeling of Polymer Matrix Nanocomposites, in: P.D. Gujrati, A.I. Leonov (Eds.) Modeling and Simulation in Polymers, Wiley VCH Verlag GmbH\&Co, Weinheim, Germany, 2010.

[16] R. Mittal, H. Dong, M. Bozkurttas, F.M. Najjar, A. Vargas, A. von Loebbecke, Journal of Computational Physics, 227 (2008) 4825-4852.

[17] H. Wan, H. Dong, Z. Liang, Vortex Formation of Freely Falling Plates in: 50th AIAA Aerospace Sciences Meeting including the New Horizons Forum and Aerospace Exposition, Nashville, Tennessee, 2011.

[18] R. Scardovelli, S. Zaleski, Annual Review of Fluid Mechanics, 31 (1999) 567-603.

[19] S. Osher, J.A. Sethian, Journal of Computational Physics, 79 (1988) 12-49.

[20] S. Chen, B. Merriman, S. Osher, P. Smereka, Journal of Computational Physics, 135 (1997) 8-29.

[21] M. Sussman, P. Smereka, S. Osher, Journal of Computational Physics, 114 (1994) 146-159.

[22] M. Sussman, E.G. Puckett, Journal of Computational Physics, 162 (2000) 301-337.

[23] D.L. Sun, W.Q. Tao, International Journal of Heat and Mass Transfer, 53 (2010) 645-655.

[24] E. Olsson, G. Kreiss, S. Zahedi, Journal of Computational Physics, 225 (2007) 785-807.

[25] P.-H. Chiu, Y.-T. Lin, Journal of Computational Physics, 230 (2011) 185-204.

[26] W.J. Boettinger, J.A. Warren, C. Beckermann, A. Karma, Annual Review of Materials

Research, 32 (2002) 163-194. 
[27] D.M. Anderson, G.B. McFadden, A.A. Wheeler, Annual Review of Fluid Mechanics, 30 (1998) 139-165.

[28] H. Wan, H. Dong, G.P. Huang, AIAA Journal, 50 (2012) 2020-2027.

[29] H. Wan, H. Dong, P. Beran, Wake Coherent Structure of Hovering Flapping Wings, in:

28th AIAA Applied Aerodynamics Conference, Chicago, Illinois, 2010.

[30] H. Wan, H. Dong, G.P. Huang, Computational Fluid-Body Interaction of Hinge Connected Flapping Plate in Hover, in: 49th AIAA Aerospace Sciences Meeting including the New

Horizons Forum and Aerospace Exposition, Orlando, Florida, 2011.

[31] H. Wan, H.B. Dong, G.P. Huang, AIAA Journal, 50 (2012) 2020-2027.

[32] H. Wan, S.S. Patnaik, Enhanced Convective Heat Transfer in Enclosures with Internal Heat Sinks, in: 11th AIAA/ASME Joint Thermophysics and Heat Transfer Conference, Atlanta, GA, 2014.

[33] E. Iskrenova, S.S. Patnaik, Transport Properties of Water and Sodium Dodecyl Sulfate, in, AFRL-RQ-WP-TP-2014-0145, 2014.

[34] O. Akin-Ojo, Y. Song, F. Wang, J. Chem. Phys., 129 (2008) 064108.

[35] A.J. Lowery, J.W. Westwater, Industrial and Engineering Chemistry, 49 (1957) 1445-1448.

[36] P.D. Jontz, J.E. Myers, AIChE Journal, 6 (1960) 34 -38.

[37] W. Frost, C.J. Kippenhan, Int. J. Heat Mass Transfer 10 (1967) 931 - 949.

[38] L. Cheng, D. Mewes, A. Luke, International Journal of Heat and Mass Transfer, 50 (2007) 2744-2771.

[39] C. Jyh-Fuh, L. Ming-Huei, Y. Yu-Min, Int. J. Heat Mass Transfer 36 (1993) 4071 - 4076.

[40] R.M. Manglik, V.M. Wasekar, J.T. Zhang, Exp. Therm. Fluid Sci., 25 (2001) 55-64.

[41] V.M. Wasekar, R.M. Manglik, Int. J. Heat Mass Transfer, 45 (2002) 483-493.

[42] G. Hetsroni, J.L. Zakin, M. Gurevich, A. Mosyak, E. Pogrebnyak, R. Rozenblit, Int. J.

Multiphase Flow 30 (2004) 717 - 734.

[43] G. Hetsroni, M. Gurevich, A. Mosyak, R. Rozenblit, Z. Segal, Int. J. Heat and Fluid Flow 25 (2004) $841-848$.

[44] J.T. Zhang, R.M. Manglik, J. Heat Transfer, 127 (2005) 684-691.

[45] V. Wasekar, Heat Mass Transfer, 45 (2009) 1409-1414.

[46] W. Cheng, B. Xie, F. Han, H. Chen, Exp. Therm. Fluid Sci., 45 (2013) 198 - 202.

[47] W.-T. Wu, H.-S. Lin, Y.-M. Yang, J.-R. Maa, Int. J. Heat Mass Transfer 37 (1994) 2377 2379.

[48] W.-T. Wu, Y.-M. Yang, J.-R. Maa, Exper. Thermal Fluid Sci. , 18 (1998) 195 - 209.

[49] W.-T. Wu, Y.-M. Yang, J.-R. Maa, Int. J. Heat Mass Transf., 42 (1999) 2483 - 2488.

[50] H. Peng, G. Ding, H. Hu, Exper. Thermal Fluid Sci. , 35 (2011) 960 - 970.

[51] Y. Xuan, Q. Li, P. Tie, Exp. Therm. Fluid Sci. , 46 (2013) 259 - 262.

[52] A.I. Morgan, L.A. Bromley, C.R. Wilke, Industrial and Engineering Chemistry, 41 (1949) 2767-2769.

[53] J. Madejski, Int. J. Heat Mass Transfer 8(1965) 155 - 171.

[54] Y.L. Tzan, Y.M. Yang, J. Heat Transf., 112 (1990) 207-212.

[55] D. Wen, G. Lin, S. Vafaei, K. Zhang, Particuology 7(2009) $141-150$.

[56] T. Inoue, M. Monde, Int. J. Heat Mass Transf., 55 (2012) 3395 - 3399.

[57] V.B. Fainerman, R. Miller, Colloids and Surfaces A: Physicochemical and Engineering Aspects 97 (1995) $65-82$.

[58] D.S. Wen, B.X. Wang, Int. J. Heat Mass Transfer 45 (2002) 1739 - 1747. 
[59] G. Hetsroni, M. Gurevich, A. Mosyak, R. Rozenblit, Int. J. Multiphase Flow 33 (2007) 1141 $-1152$.

[60] M. Chandrasekar, S. Suresh, T. Senthilkumar, Renew. Sustain. Energy Rev. , 16 (2012) 3917 - 3938.

[61] Z. Mingzheng, X. Guodong, L. Jian, C. Lei, Z. Lijun, Exp. Therm. Fluid Sci., 36 (2012) 22 29.

[62] H.Y. Yoon, S. Koshizuka, Y. Oka, Int. J. Multiphase Flow 27 (2001) 277 - 298.

[63] I. Sher, G. Hetsroni, Int. J. Multiphase Flow, 28 (2002) 699 - 706.

[64] Y.-Y. Li, Z.-H. Liu, G.-S. Wang, Int. J. Heat Mass Transf., 65 (2013) 789 - 797.

[65] W.L. Jorgensen, J. Chandrasekhar, J.D. Madura, R.W. Impey, M.L. Klein, Chem. Phys., 79 (1983) 926.

[66] Y.-P. Liu, K. Kim, B.J. Berne, R.A. Friesner, S.W. Rick, J. Chem. Phys., 108 (1998) 47394755.

[67] J. Guillot, J. Mol. Liq., 101 (2002) 219-260.

[68] C.J. Burnhan, S.S. Xantheas, J. Chem. Phys., 116 (2002) 5115-5124.

[69] D.M. Anderson, G.B. McFadden, Physics of Fluids, 9 (1997) 1870-1879.

[70] C. Vega, E. de Miguel, J. Chem. Phys., 126 (2007) 154707.

[71] J.-P. Ryckaert, G. Ciccotti, H.J.C. Berendsen, J. Comput. Phys., 23 (1977) 327.

[72] I.G. Tironi, R.M. Brunne, W.F. van Gunsteren, Chem. Phys. Lett., 250 (1996) 19 - 24.

[73] W.D. Cornell, P. Cieplak, C.I. Bayly, I.R. Gould, K.M. Merz, D.M. Ferguson, D.C.

Spellmeyer, T. Fox, J.W. Caldwell, P.A. Kollman, J. Am. Chem. Soc., 117 (1995) 5179-5197. [74] A.D. MacKerell, D. Bashford, D. Bellott, R. L., , J.D. Evanseck, M.J. Field, S. Fischer, J. Gao, H. Guo, S. Ha, D. Joseph-McCarthy, L. Kuchnir, K. Kuczera, F.T.K. Lau, C. Mattos, S. Michnick, T. Ngo, D.T. Nguyen, B. Prodhom, Reiher, W. E., , B. Roux, M. Schlenkrich, J.C. Smith, R. Stote, J. Straub, M. Watanabe, J. Wirkiewicz-Kuczera, D. Yin, M. Karplus, J. Phys. Chem. B, 102 (1998) 3586-3616.

[75] W.L. Jorgensen, D.S. Maxwell, J. Tirado-Rives, Journal of the American Chemical Society, 118 (1996) 11225-11236.

[76] R.T. Cygan, J.-J. Liang, A.G. Kalinichev, J. Phys. Chem. B, 108 (2004) 1255-1266.

[77] Y. Mao, Y. Zhang, Chem. Phys. Lett., 542 (2012) 37 - 41.

[78] D. van der Spoel, P.J. van Maaren, H.J.C. Berendsen, J. Chem. Phys., 108 (1998) 10220.

[79] P. Mark, L. Nilsson, J. Phys. Chem. A, 105 (2001) 9954.

[80] D.J. Price, C.L. Brooks III, J. Chem. Phys., 121 (2004) 10096.

[81] Y. Wu, H.L. Tepper, G.A. Voth, J. Chem. Phys., 124 (2006) 024503.

[82] T.W. Sirk, S. Moore, E.F. Brown, J. Chem. Phys., 138 (2013) 064505.

[83] K.J. Schweighofer, U. Essmann, M. Berkowitz, J. Phys. Chem. B, 101 (1997) 3793.

[84] F. Ercolessi, J.B. Adams, Europhys. Lett., 26 (1994) 583.

[85] G. Csanyi, T. Albaret, M. Payne, A. De Vita, Phys. Rev. Lett., 93 (2004).

[86] H. Dominguez, M.L. Berkowitz, J. Phys. Chem. B, 104 (2000) 5302-5308.

[87] C.D. Bruce, M.L. Berkowitz, L. Perera, M.D.E. Forbes, J. Phys. Chem. B, 106 (2002) 37883793.

[88] T. Cheng, Q. Chen, F. Li, H. Sun, J. Phys. Chem. B, 114 (2010) 13736-13744.

[89] L. Martínez, R. Andrade, E.G. Birgin, J.M. Martínez, Journal of Computational Chemistry, 30 (2009) 2157.

[90] R.W. Hockney, J.W. Eastwood, Computer simulation using particles, 1988.

[91] S. Plimpton, J. Comp. Phys., 117 (1995) 1-19. 
[92] D.M. York, T.A. Darden, L.G. Pedersen, J. Chem. Phys., 99 (1993) 8345-8348.

[93] W. Humphrey, A. Dalke, K. Schulten, J. Molev. Graphics, 14 (1996) 33.

[94] W.T. Ashurst, W.G. Hoover, Phys. Rev. A, 11 (1975) 658-678.

[95] F. Muller-Plathe, J. Chem. Phys., 106 (1997) 6082.

[96] M.M. Zhang, E. Lussetti, L.E.S. de Souza, F. Muller-Plathe, J. Phys. Chem. B, 109 (2005) 15060-15067.

[97] C.D. Bruce, S. Senapati, M.L. Berkowitz, L. Perera, M.D.E. Forbes, J. Phys. Chem. B, 106 (2002) 10902-10907.

[98] R.M. Weinheimer, D.F. Evans, E.L. Cussler, J. Colloid and Interface Sci., 80 (1981) 357.

[99] S. Izvekov, M. Parrinello, C. Burnham, G. Voth, J. Chem. Phys., 120 (2004) 10896-10913.

[100] B. D।"unweg, K. Kremer, J. Chem. Phys., 99 (1993) 6983-6997.

[101] I.C. Yeh, G. Hummer, J. Phys. Chem. B, 108 (2004) 15873-15879.

[102] S. Tazi, A. Botan, M. Salanne, V. Marry, P. Turq, B. Rotenberg, J. Phys.: Condens. Matter, 24 (2012).

[103] K. Krynicki, C.D. Green, D.W. Sawyer, Faraday Discuss. Chem. Soc., 66 (1978) 199-208.

[104] K.R. Harris, L.A. Woolf, J. Chem. Eng. Data, 49 (2004) 1064-1069.

[105] H.J.C. Berendsen, J.R. Grigera, T.P. Straatsma, J. Phys. Chem., 91 (1987) 6269-6271.

[106] J.L.F. Abascal, C. Vega, J. Chem. Phys., 123 (2005) 234505.

[107] D.R. Lide, CRC Handbook of chemistry and physics, CRC Pres, Boca Raton, FL, 2001.

[108] M.L.V. Ramires, C.A.N. de Castro, Y. Nagazaka, A. Nagashima, M.J. Assael, W.A.

Wakeham, J. Phys. Chem. Ref. Data, 24 (1995) 1377-1381.

[109] D. Johnson, S.S. Patnaik, J. Ervin, An integrated chemica reactor heat exchanger based on ammonium carbamate, in: Proceedings of the SAE 2012 Power Systems Conference, SAE International, Phoenix, 2012, pp. 2012-2001-2190.

[110] E.K. Iskrenova, S.S. Patnaik, Int. J. Heat Mass Transfer, In press (2016).

[111] J.E. Schmidt, D.S. Dudis, D.J. Miller, J. Thermophys. Heat Transfer, 26 (2012) 345-351.

[112] J.D. Johnson, J. Ervine, M. Hanchak, S.S. Patnaik, X. Hu, Journal of Thermophysics and

Heat Transfer, Accpeted (2014).

[113] N. Niedbalski, D. Johnson, S.S. Patnaik, D. Banerjee, Int. J. Heat Mass Transf., 70 (2014) 1078-1085.

[114] N. Niedbalski, D. Johnson, S.S. Patnaik, D. Banerjee, Int. J. Heat Mass Transf., 70 (2014) 1086-1094.

[115] B. Claudel, L. Boulamri, Thermochim. Acta 126 (1988) 129 - 148.

[116] B.R. Ramachandran, A.M. Halpern, E.D. Glendening, J. Phys. Chem. A, 102 (1998) 39343941.

[117] N. Wen, M.H. Brooker, J. Chem. Phys., 99 (1995) 359-368.

[118] H. Bai, A.C. Yeh, Ind. Eng. Chem. Res., 36 (1997) 2490-2493.

[119] A. Yeh, H. Bai, Sci. Total Environ., 228 (1999) 121-133.

[120] J.e.D. Figueroa, T. Fout, S. Plasynski, H. McIlvried, R.D. Srivastava, Int. J. Greenhouse

Gas Control 2(2008) 9 - 20.

[121] C.M. White, B.R. Strazisar, E.J. Granite, J.S. Hoffman, H.W. Pennline, J. Air Waste

Manag. Assoc., 53 (2003) 645-715.

[122] S.M. Benson, D.R. Cole, Elements, 4 (2008) 325-331.

[123] J. Rosenqvist, A.D. Kilpatrick, B.W.D. Yardley, Applied Geochemistry 27 (2012) 1610 1614. 
[124] Y.-F. Diao, X.-Y. Zheng, B.-S. He, C.-H. Chen, X.-C. Xu, En. Conv. Manag., 45 (2004) $2283-2296$.

[125] W. Conway, X. Wang, D. Fernandes, R. Burns, G. Lawrance, G. Puxty, M. Maeder, J. Phys. Chem. A, 115 (2011) 14340-14349.

[126] X. Wang, W. Conway, D. Fernandes, G. Lawrence, R. Burns, G. Puxty, M. Maeder, J. Phys. Chem. A, 115 (2011) 6405-6412.

[127] P.W.J. Derks, G.F. Versteeg, En. Procedia 1(2009) 1139 - 1146.

[128] J. Liu, S. Wang, B. Zhao, H. Tong, C. Chen, En. Procedia 1(2009) 933 - 940.

[129] F. Qin, S. Wang, A. Hartono, H.F. Svendsen, C. Chen, Int. J. of Greenhouse Gas Control 4(2010) $729-738$.

[130] J. Liu, S. Wang, G. Qi, B. Zhao, C. Chen, Energy Procedia 4(2011) 525 - 532.

[131] S.-B. Jeon, J.-B. Seo, H.-D. Lee, S.-K. Kang, K.-J. Oh, Kor. J. Chem. Eng., 30 (2013)

1790-1796.

[132] M. Caplow, J. Am. Chem. Soc., 90 (1968) 6795-6803.

[133] P.V. Danckwerts, Chem. Eng. Sci., 34 (1979) 443 - 446.

[134] B.G. Choi, H.S. Park, G.H. Kim, Y.M. Jung, K.B. Yi, J.-N. Kim, W.H. Hong, J. Ind. Eng. Chem., 18 (2012) 98 - 104.

[135] B. Zhao, Y. Su, W. Tao, L. Li, Y. Peng, Int. J. Greenhouse Gas Control 9(2012) 355 - 371. [136] G. Puxty, R. Rowland, M. Attalla, Chem. Eng. Sci., 65 (2010) 915 - 922.

[137] C. Cadena, J.L. Anthony, J.K. Shah, T.I. Morrow, J.F. Brennecke, E.J. Maginn, Journal of the American Chemical Society, 126 (2004) 5300-5308.

[138] D.M. D'Alessandro, B. Smit, J.R. Long, Angew. Chem. Int. Ed., 49 (2010) 6058-6082.

[139] J.F. Brennecke, B.E. Gurkan, J. Phys. Chem. Lett., 1 (2010) 3459-3464.

[140] M.B. Shiflett, A. Yokozeki, Ind. Eng. Chem. Res., 44 (2005) 4453-4464.

[141] J. Li, C. You, L. Chen, Y. Ye, Z. Qi, K. Sundmacher, Ind. Eng. Chem. Res., 51 (2012) 12081-12088.

[142] C. Guo, S. Chen, Y. Zhang, G. Wang, J. Chem. Eng. Data, 59 (2014) 1796-1801.

[143] L. Saiz, J.A. Padrl'o, E. Gul'ardia, J. Chem. Phys., 114 (2001) 3187-3199.

[144] M. Szczerba, Z. Kllapyta, A. Kalinichev, Appl. Clay Sci., 91 (2014) 87 - 97.

[145] A. Kaiser, O. Ismailova, A. Koskela, S.E. Huber, M. Ritter, B. Cosenza, W. Benger, R.

Nazmutdinov, M. Probst, J. Molec. Liquids 189 (2014) 20 - 29.

[146] W. Damm, A. Frontera, J. Tirado-Rives, W. Jorgensen, J. Comput. Chem., 18 (1997)

1955-1970.

[147] R.C. Rizzo, W.L. Jorgensen, J. Am. Chem. Soc., 121 (1999) 4827-4836.

[148] O.V. de Oliveira, L.C.G. Freitas, J. Mol. Structure: THEOCHEM 728 (2005) 179 - 187.

[149] V. Hornak, R. Abel, A. Okur, B. Strockbine, A. Roitberg, C. Simmerling, Proteins:

Structure, Function, and Bioinformatics, 65 (2006) 712-725.

[150] O.A. Moultos, I.N. Tsimpanogiannis, A.Z. Panagiotopoulos, I.G. Economou, J. Chem.

Phys. B, 118 (2014) 5532-5541.

[151] M. Garcia-Rates, J.-C. de Hemptinne, J.B. Avalos, C. Nieto-Draghi, J. Phys. Chem. B, 116 (2012) 2787-2800.

[152] L.J. Criscenti, R.T. Cygan, Environ. Sci. Technol., 47 (2013) 87-94.

[153] T.C. Lourenlcco, M.F.C. Coelho, T.C. Ramalho, D. van der Spoel, L.T. Costa, Environ.

Sci. Technol., 47 (2013) 7421-7429.

[154] R.T. Cygan, V.N. Romanov, E.M. Myshakin, J. Chem. Phys. C, 116 (2012) 13079-13091.

[155] J.G. Harris, K.H. Yung, J. Chem. Phys., 99 (1995) 12021-12024.

DISTRIBUTION STATEMENT A: Approved for public release. Distribution is unlimited. 
[156] R.W. Impey, M.L. Klein, Chem. Phys. Lett., 104 (1984) 579-582.

[157] L. Zhang, J.I. Siepmann, Coll. Czech. Chem. Comm., 75 (2010) 577-591.

[158] J.C. de la Fuente Badilla, C.J. Peters, J. de Swaan Arons, J. Supercrit. Fluids 17 (2000) 13 23.

[159] I. Short, A. Sahgal, W. Hayduk, J. Chem. Eng. Data, 28 (1983) 63-66.

[160] M.I. Pownceby, D.H. Jenkins, R. Ruzbacky, S. Saunders, J. Chem. Eng. Data, 57 (2012) 1449-1455.

[161] G. Nagayama, M. Takematsu, H. Mizuguchi, T. Tsuruta, J. Chem. Phys., 143 (2015) 014706.

[162] J. Vieceli, M. Roeselovl'a, D.J. Tobias, Chem. Phys. Lett. , 393 (2004) 249 - 255.

[163] B.C. Garrett, G.K. Schenter, A. Morita, Chem. Rev., 106 (2006) 1355-1374.

[164] G. Murdachaew, M.E. Varner, L.F. Phillips, B.J. Finlayson-Pitts, R.B. Gerber, Physical Chemistry Chemical Physics, 15 (2013) 204-212.

[165] G. Nagayama, M. Kawagoe, A. Tokunaga, T. Tsuruta, Int. J. Thermal Sci., 49 (2010) 5966.

[166] J. Yu, H. Wang, Int. J. Heat Mass Transf., 55 (2012) 1218-1225.

[167] Z. Liang, P. Keblinski, Int. J. Heat Mass Transf. , 78 (2014) 161 - 169.

[168] T. Tsuruta, H. Tanaka, T. Masuoka, Int. J. Heat Mass Transf. , 42 (1999) 4107 - 4116.

[169] T. Tsuruta, G. Nagayama, Energy 30 (2005) 795 - 805.

[170] T. Tsuruta, G. Nagayama, J. Phys. Chem. B, 108 (2004) 1736-1743.

[171] P.G. Bolhuis, D. Chandler, C. Dellago, P.L. Geissler, Ann. Rev. Phys. Chem., 53 (2002) 291-318.

[172] G. Nagayama, T. Tsuruta, J. Chem. Phys., 118 (2003) 1392-1399.

[173] A. Morita, M. Sugiyama, H. Kameda, S. Koda, D.R. Hanson, J. Phys. Chem. B, 108 (2004) 9111-9120.

[174] J. Julin, M. Shiraiwa, R.E.H. Miles, J.P. Reid, U. P\"oschl, I. Riipinen, J. Phys. Chem. A, 117 (2013) 410-420.

[175] P. Varilly, D. Chandler, J. Phys. Chem. B, 117 (2013) 1419-1428.

[176] B.-Y. Cao, J.-F. Xie, S.S. Sazhin, J. Chem. Phys., 134 (2011) 164309.

[177] J.-F. Xie, S.S. Sazhin, B.-Y. Cao, Phys. Fluids 23 (2011) 112104.

[178] Y. Wang, P. Keblinski, Journal of Applied Physics, 111 (2012) 064321.

[179] M. Macdonald, S. Garimella, International Journal of Heat and Mass Transfer, 93 (2016) $75-85$.

[180] G. Kaminski, W.L. Jorgensen, Performance of the AMBER94, MMFF94, and OPLS-AA force fields for modeling organic liquids, in: J. Phys. Chem., 1996, pp. 18010-18013.

[181] M.G. Martin, J.I. Siepmann, J. Phys. Chem. B, 102 (1998) 2569-2577.

[182] M.S. Kelkar, J.L. Rafferty, E.J. Maginn, J.I. Siepmann, Fluid Phase Equil., 260 (2007) 218-231.

[183] T.H. Yang, C. Pan, International Journal of Heat and Mass Transfer, 48 (2005) 3516-3526. [184] J. Vieceli, M. Roeselová, N. Potter, L.X. Dang, B.C. Garrett, D.J. Tobias, The Journal of Physical Chemistry B, 109 (2005) 15876-15892.

[185] M.S. Hanchak, M.D. Vangsness, L.W. Byrd, J.S. Ervin, Int. J. Heat Mass Transf. , 75 (2014) 196 - 206.

[186] R. Marek, J. Straub, International Journal of Heat and Mass Transfer, 44 (2001) 39-53.

[187] M. Matsumoto, Y. Kataoka, The Journal of Chemical Physics, 88 (1988) 3233-3245.

[188] L.X. Dang, T.-M. Chang, The Journal of Chemical Physics, 106 (1997) 8149-8159. 
[189] O. Mesalhy, K. Lafdi, A. Elgafy, Carbon, 44 (2006) 2080-2088.

[190] O. Mesalhy, K. Lafdi, A. Elgafy, K. Bowman, Energy Conversion and Management, 46 (2005) 847-867.

[191] X. Hu, S.S. Patnaik, Modeling Phase Change Material in Microfoam Under Constant

Temperature Condition, in, AFRL-RQ-WP-TP-2014-0026, 2014.

[192] X. Hu, S. Patnaik, S., International Journal of Heat and Mass Transfer, 68 (2014) 677-682.

[193] A.M. Druma, M.K. Alam, C. Druma, Int J Therm Sci, 43 (2004) 689-695.

[194] S. Krishnan, J.Y. Murthy, S.V. Garimella, Journal of Heat Transfer, 128 (2006) 793-799.

[195] S. Krishnan, S.V. Garimella, J.Y. Murthy, Journal of Heat Transfer, 130 (2008) 024503.

[196] K. Lafdi, O. Mesalhy, S. Shaikh, Journal of Applied Physics, 102 (2007) 083549.

[197] V.V. Calmidi, R.L. Mahajan, Journal of Heat Transfer, 121 (1999) 466-471.

[198] J.W. Paek, B.H. Kang, S.Y. Kim, J.M. Hyun, International Journal of Thermophysics, 21 (2000) 453-464.

[199] A. Bhattacharya, V.V. Calmidi, R.L. Mahajan, International Journal of Heat and Mass

Transfer, 45 (2002) 1017-1031.

[200] B. Maruyama, J.E. Spowart, D.J. Hooper, H.M. Mullens, A.M. Druma, C. Druma, M.K. Alam, Scripta Materialia, 54 (2006) 1709-1713.

[201] K.K. Bodla, J.Y. Murthy, S.V. Garimella, Numerical Heat Transfer, Part A: Applications: An International Journal of Computation and Methodology, 58 (2010) 527-544.

[202] K.K. Bodla, J.Y. Murthy, S.V. Garimella, Computational Materials Science, 50 (2010) $622-632$.

[203] X. Hu, S.S. Patnaik, 43rd AIAA Thermophysics Conference, (2012).

[204] H. Mehling, L.F. Cabeza, Heat and Cold Storage with PCM: An up to Date Introduction into Basics and Applications, Springer, 2008.

[205] V. Shatikian, G. Ziskind, R. Letan, International Journal of Heat and Mass Transfer, 48 (2005) 3689-3706.

[206] V. Shatikian, G. Ziskind, R. Letan, International Journal of Heat and Mass Transfer, 51 (2008) 1488-1493.

[207] X.-Q. Wang, A.S. Mujumdar, C. Yap, International Communications in Heat and Mass Transfer, 34 (2007) 801-808.

[208] X.-Q. Wang, C. Yap, A.S. Mujumdar, Int J Therm Sci, 47 (2008) 1055-1068.

[209] R. Kandasamy, X.-Q. Wang, A.S. Mujumdar, Applied Thermal Engineering, 28 (2008) 1047-1057.

[210] C. Beckermann, R. Viskanta, International Journal of Heat and Mass Transfer, 31 (1988) $35-46$.

[211] A. Erek, I. Dincer, International Journal of Heat and Mass Transfer, 52 (2009) 851-859.

[212] M.N.A. Hawlader, M.S. Uddin, H.J. Zhu, International Journal of Energy Research, 26

(2002) 159-171.

[213] K. Vafai, C.L. Tien, International Journal of Heat and Mass Transfer, 25 (1980) 1183-

1190 .

[214] J. Klett, R. Hardy, E. Romine, C. Walls, T. Burchell, Carbon, 38 (2000) 953-973.

[215] S.-T. Hong, D.R. Herling, Scripta Materialia, 55 (2006) 887-890.

[216] S.T. Hong, D.R. Herling, Advanced Engineering Materials, 9 (2007) 554-557.

[217] T.H. Bauer, International Journal of Heat and Mass Transfer, 36 (1993) 4181-4191.

[218] S. Krishnan, S.V. Garimella, J.Y. Murthy, Journal of Heat Transfer-Transactions of the Asme, 130 (2008).

DISTRIBUTION STATEMENT A: Approved for public release. Distribution is unlimited. 
[219] V.V. Calmidi, R.L. Mahajan, Journal of Heat Transfer, 122 (2000) 557-565.

[220] E.M. Alawadhi, C.H. Amon, IEEE Transactions on Components and Packaging Technologies, 26 (2003) 116-125.

[221] W.J. Minkowycz, A. Haji-Sheikh, K. Vafai, International Journal of Heat and Mass

Transfer, 42 (1999) 3373-3385.

[222] S. Krishnan, J.Y. Murthy, S.V. Garimella, Journal of Heat Transfer, 127 (2005) 995-1004.

[223] S. Krishnan, J.Y. Murthy, S.V. Garimella, Asme, Journal of Heat Transfer, 129 (2007) 395-400.

[224] M. Almajali, K. Lafdi, S. Shaikh, Journal of Applied Physics, 102 (2007) 033506-033506033508 .

[225] K. Lafdi, O. Mesalhy, A. Elgafy, Journal of Electronic Packaging, 130 (2008) 021004-

021008.

[226] V.R. Voller, C. Prakash, International Journal of Heat and Mass Transfer, 30 (1987) 17091719.

[227] R. Lewis, P. Nithiarasu, K. Seetharamu, Fundamentals of the finite element method for heat and fluid flow, Wiley, 2004.

[228] D. Poulikakos, A. Bejan, Physics of Fluids, 26 (1983) 1183-1191.

[229] M. Lappa, Thermal Convection : Patterns, Evolution and Stability, John Wiley and Sons, 2009.

[230] E.P. van der Poel, R.J.A.M. Stevens, K. Sugiyama, D. Lohse, Physics of Fluids, 24 (2012) 085104.

[231] T.P. Lyubimova, D.V. Lyubimov, V.A. Morozov, R.V. Scuridin, H. Ben Hadid, D. Henry, J Fluid Mech, 635 (2009) 275-295.

[232] R.M. Kerr, J Fluid Mech, 419 (2000) 325-344.

[233] J.M. Hyun, Journal of Heat Transfer, 107 (1985) 982-984.

[234] S. Wagner, O. Shishkina, Physics of Fluids, 25 (2013) 085110.

[235] J. Patterson, J. Imberger, J Fluid Mech, 100 (1980) 65-86.

[236] M. Corcione, Int J Therm Sci, 42 (2003) 199-208.

[237] I.B. Palymskii, Fluid Dyn, 42 (2007) 550-559.

[238] O. Turan, R.J. Poole, N. Chakraborty, International Journal of Heat and Fluid Flow, 33 (2012) 131-146.

[239] J.M. House, C. Beckermann, T.F. Smith, Numerical Heat Transfer, Part A: Applications, 18 (1990) 213-225.

[240] J.R. Lee, M.Y. Ha, S. Balachandar, H.S. Yoon, S.S. Lee, Physics of Fluids, 16 (2004) 1097-1117.

[241] D. Angeli, P. Levoni, G.S. Barozzi, International Journal of Heat and Mass Transfer, 51 (2008) 553-565.

[242] B.S. Kim, D.S. Lee, M.Y. Ha, H.S. Yoon, International Journal of Heat and Mass Transfer, 51 (2008) 1888-1906.

[243] M.Y. Ha, I.K. Kim, H.S. Yoon, S. Lee, Physics of Fluids, 14 (2002) 3189-3202.

[244] G. Cesini, M. Paroncini, G. Cortella, M. Manzan, International Journal of Heat and Mass Transfer, 42 (1999) 1801-1811.

[245] H.S. Yoon, J.H. Jung, Y.G. Park, Numerical Heat Transfer, Part A: Applications, 62 (2012) 701-721.

[246] J.R. Lee, I.S. Park, Nucl Eng Technol, 44 (2012) 283-296. 
[247] P. Bhave, A. Narasimhan, D.A.S. Rees, International Journal of Heat and Mass Transfer, 49 (2006) 3807-3818.

[248] G. De Vahl Davis, International Journal for Numerical Methods in Fluids, 3 (1983) 249264.

[249] H.N. Dixit, V. Babu, International Journal of Heat and Mass Transfer, 49 (2006) 727-739. [250] A.E. Gill, J Fluid Mech, 26 (1966) 515-536.

[251] D.E. Cormack, L.G. Leal, J.H. Seinfeld, J Fluid Mech, 65 (1974) 231-246.

[252] I. Mercader, O. Batiste, L. Ramirez-Piscina, X. Ruiz, S. Rudiger, J. Casademunt, Physics of Fluids, 17 (2005).

[253] G. Huelsz, R. Rechtman, Int J Therm Sci, 65 (2013) 111-119.

[254] J. Mizushima, T. Adachi, Journal of the Physical Society of Japan, 64 (1995) 4680-4683.

[255] S. Arcidiacono, I. Di Piazza, M. Ciofalo, International Journal of Heat and Mass Transfer, 44 (2001) 537-550.

[256] M.G. Braunsfurth, A.C. Skeldon, A. Juel, T. Mullin, D.S. Riley, J Fluid Mech, 342 (1997) 295-314.

[257] B. Hof, A. Juel, L. Zhao, D. Henry, H. Ben Hadid, T. Mullin, J Fluid Mech, 515 (2004)

391-413.

[258] A. Juel, T. Mullin, H. Ben Hadid, D. Henry, J Fluid Mech, 436 (2001) 267-281.

[259] M.M. Zdravkovich, Journal of Wind Engineering and Industrial Aerodynamics, 7 (1981) 145-189.

[260] T. Sarpkaya, J Fluid Struct, 19 (2004) 389-447.

[261] J.C. Owen, P.W. Bearman, A.A. Szewczyk, J Fluid Struct, 15 (2001) 597-605.

[262] F.S. Hover, H. Tvedt, M.S. Triantafyllou, J Fluid Mech, 448 (2001) 175-195.

[263] P.J. Strykowski, K.R. Sreenivasan, J Fluid Mech, 218 (1990) 71-107.

[264] K. Kwon, H. Choi, Physics of Fluids, 8 (1996) 479-486.

[265] W.L. Chen, D.B. Xin, F. Xu, H. Li, J.P. Ou, H. Hu, J Fluid Struct, 42 (2013) 25-39.

[266] D.R. Arcas, L.G. Redekopp, Physics of Fluids, 16 (2004) 452-456.

[267] J.E. Ffowcs Williams, B.C. Zhao, J Fluid Struct, 3 (1989) 115-122.

[268] S. Hiejima, T. Nomura, K. Kimura, Y. Fujino, Journal of Wind Engineering and Industrial Aerodynamics, 67-8 (1997) 325-335.

[269] N. Fujisawa, G. Takeda, J Fluid Struct, 17 (2003) 903-913.

[270] H. Wan, S.P. Soumya, A numerical study of thermal effects on vortex-induced vibration,

in: 53rd AIAA Aerospace Sciences Meeting, Florida, 2015.

[271] H. Wan, S.S. Patnaik, Numerical Study of Thermal Effects on Vortex-induced Vibrations in, AFRL-RQ-WP-TP-2016-0084, 2016.

[272] H.T. Ahn, Y. Kallinderis, J Comput Phys, 219 (2006) 671-696.

[273] C.H.K. Williamson, R. Govardhan, Annu Rev Fluid Mech, 36 (2004) 413-455.

[274] D. Brika, A. Laneville, J. Fluid Mech., 250 (1993) 481-508. 